Portland State University

PDXScholar

Spring 6-4-2019

\title{
Seismic Performance of Substandard Reinforced Concrete Bridge Columns under Subduction-Zone Ground Motions
}

Alvaro Francisco Lopez Ibaceta

Portland State University

Follow this and additional works at: https://pdxscholar.library.pdx.edu/open_access_etds

Part of the Civil and Environmental Engineering Commons

Let us know how access to this document benefits you.

\section{Recommended Citation}

Lopez Ibaceta, Alvaro Francisco, "Seismic Performance of Substandard Reinforced Concrete Bridge Columns under Subduction-Zone Ground Motions" (2019). Dissertations and Theses. Paper 4977. https://doi.org/10.15760/etd.6853

This Dissertation is brought to you for free and open access. It has been accepted for inclusion in Dissertations and Theses by an authorized administrator of PDXScholar. Please contact us if we can make this document more accessible: pdxscholar@pdx.edu. 
Seismic Performance of Substandard Reinforced Concrete Bridge Columns under Subduction-Zone Ground Motions

by

Álvaro Francisco López Ibaceta

A dissertation submitted in partial fulfillment of the requirements for the degree of

\author{
Doctor of Philosophy \\ in \\ Civil and Environmental Engineering
}

Dissertation Committee:

Peter Dusicka, Chair

Franz Rad

Thomas Schumacher

Scott Burns

Portland State University
2019 
(C) 2019 Alvaro Francisco Lopez Ibaceta 


\section{ABSTRACT}

A large magnitude, long duration subduction earthquake is impending in the Pacific Northwest, which lies near the Cascadia Subduction Zone (CSZ). Great subduction zone earthquakes are the largest earthquakes in the world and are the sole source zones that can produce earthquakes greater than M8.5. Additionally, the increased duration of a CSZ earthquake may result in more structural damage than expected. Given such seismic hazard, the assessment of reinforced concrete substructures has become crucial in order to prioritize the bridges that may need to be retrofitted and to maintain the highway network operable after a major seismic event. Recent long duration subduction earthquakes occurred in Maule, Chile $\left(\mathrm{M}_{\mathrm{w}} 8.8,2010\right)$ and Tohoku, Japan $\left(\mathrm{M}_{\mathrm{w}} 9.0,2011\right)$ are a reminder of the importance of studying the effect of subduction ground motions on structural performance. For this purpose, the seismic performance of substandard circular reinforced concrete bridge columns was experimentally evaluated using shake table tests by comparing the column response from crustal and subduction ground motions. Three continuous reinforced columns and three lap-spliced columns were tested using records from 1989 Loma Prieta, 2010 Maule and 2011 Tohoku. The results of the large-scale experiments and numerical studies demonstrated that the increased duration of subduction ground motions affects the displacement capacity and can influence the failure mode of bridge columns. Furthermore, more damage was recorded under the subduction ground motions as compared to similar maximum deformations under the crustal ground motion. The larger number of plastic strain cycles imposed by subduction 
ground motions influence occurrence of reinforcement bar buckling at lower displacement compared to crustal ground motions. Moreover, based on the experimental and numerical results, subduction zone ground motion effects are considered to have a significant effect on the performance of bridge columns. Therefore, it is recommended to consider the effects of subduction zone earthquakes in the performance assessment of substandard bridges, or when choosing ground motions for nonlinear time-history analysis, especially in regions prone to subduction zone mega earthquakes. Finally, for substandard bridges not yet retrofitted or upgraded seismically, the following performance limit recommendation is proposed: for the damage state of collapse, which is related to the ODOT's Life Safety performance level, the maximum strain in the longitudinal reinforcement should be reduced from 0.09 (in./in.) to a value of 0.032 (in./in.) for locations where subduction zone earthquakes are expected, to take into consideration the occurrence of bar buckling. 
To my family 


\section{ACKNOWLEDGMENTS}

This research was made possible through the support, guidance, and encouragement of many individuals and institutions.

I would like first to express my appreciation and gratitude to my advisor, Dr. Peter Dusicka for his guidance and support throughout my time at Portland State University. I would also like to thank my doctoral committee members, Dr. Franz Rad, Dr. Thomas Schumacher, and Dr. Scott Burns for the time dedicated on reviewing the work presented here, but also in other activities during my time at PSU.

This research project was financially supported by the Oregon Department of Transportation (ODOT) through the project SPR\#770. I want to thank Conicyt and Universidad Tecnica Federico Santa Maria for providing the grants to stay at Portland State University.

Next, I would like to express the most profound gratitude to Megan Falcone for all her help and assistance throughout my PhD studies. Also, I express my gratitude and friendship to Ilya Palnikov for his help and dedicated assistance during the course of the shake table experiments. Also, my heartfelt thanks to Aaron Smith, Seth Eisele, Carrington Crites, Anwer Mohammed, Patrick McCoy, Ke Liilah Vara, Tyler Williams, Dr. Arlindo Lopes, Dr. Selamawit Mehary, Dr. Ramiro Bazaez, Stephen Smithson and many others for the help provided and the enjoyable and cheerful conversations during the time of studies at Portland.

This acknowledgment would not be completed without expressing gratitude to my family. My lovely wife Carla, my daughter Martina and son Matias are my inspiration. I 
am eternally grateful for their confidence, love, unlimited emotional support and encouragement. My deepest gratitude to my parents, Francisco and Fresia, for their love, emotional support, and encouragement. I would also like to thank my two sisters Francisca and Maria Jose for their emotional support and encouragement. Also, thanks to my in-laws for their encouragement. Finally, thanks to God for always protecting my family and me, and guiding me through all this time. 


\section{TABLE OF CONTENTS}

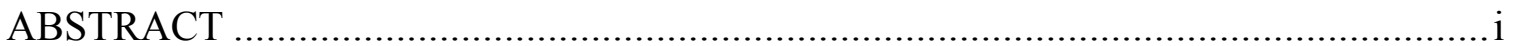

DEDICATION .............................................................................................

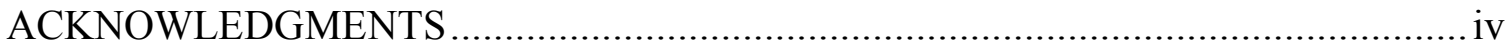

LIST OF TABLES ..................................................................................

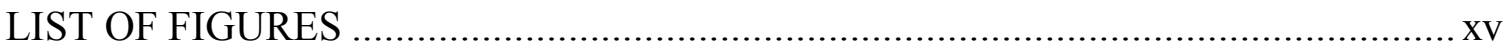

CHAPTER 1. Introduction and Previous Research .................................................. 1

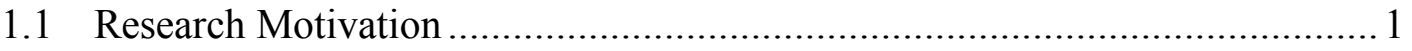

1.2 Subduction-Zone Megathrust Earthquakes ........................................... 2

1.2.1 Chile Subduction Zone ................................................................ 4

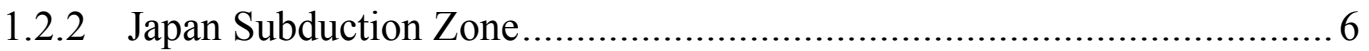

1.2.3 Cascadia Subduction Zone......................................................... 7

1.3 Previous Research Including Effect of Subduction Zone Earthquakes ............ 8

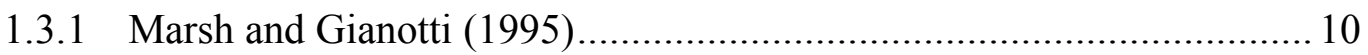

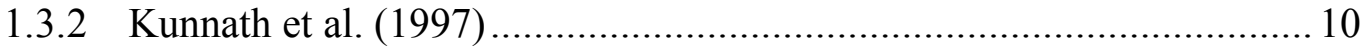

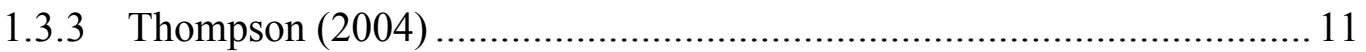

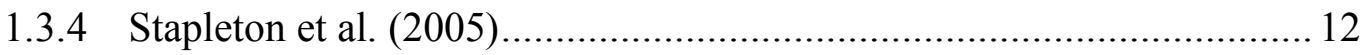

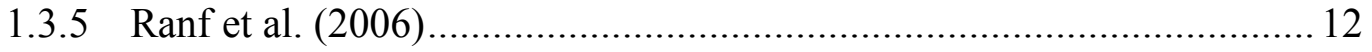

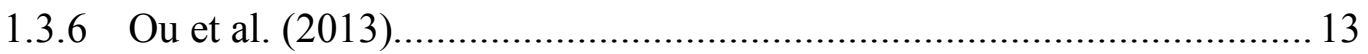

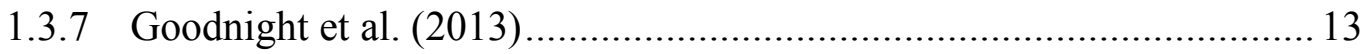

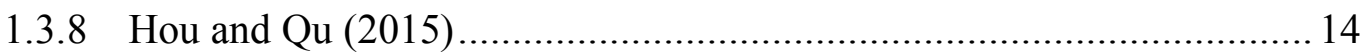

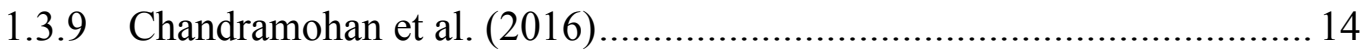




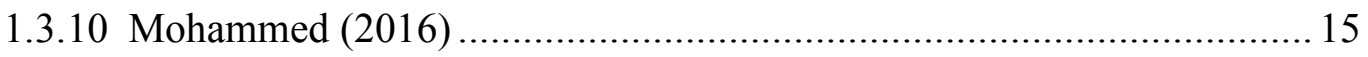

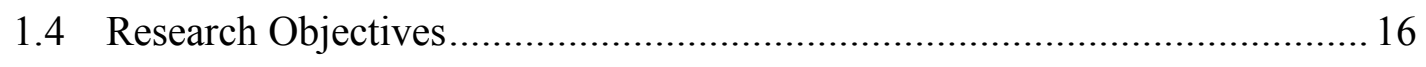

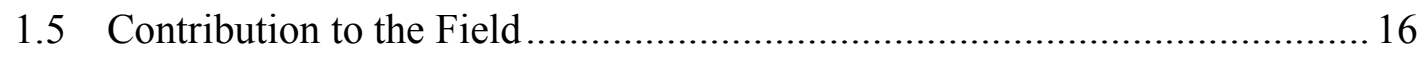

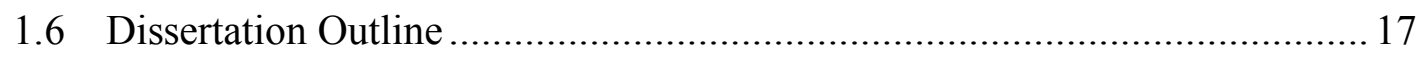

1.7 Retrofit Strategies for RC Bridge Columns ……………………................ 18

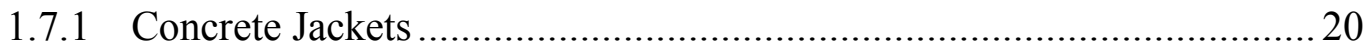

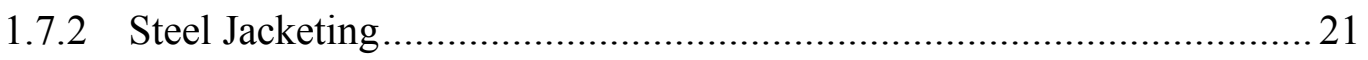

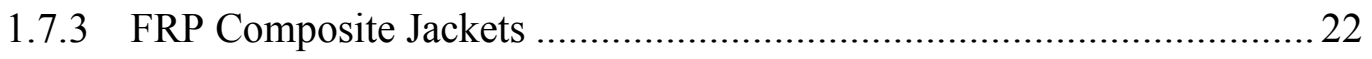

1.7.4 External Prestressing Steel............................................................... 23

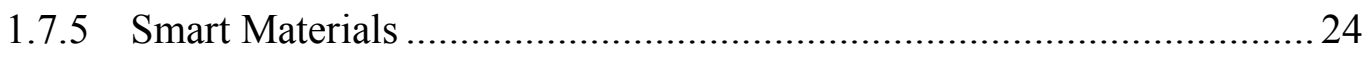

1.8 Lessons from Recent Subduction Zone Earthquakes …………................... 24

1.8.1 Damage in Bridges due to the 2010 Chile earthquake. ..........................25

1.8.2 Damage in Bridges due to the 2011 Tohoku earthquake.........................28

CHAPTER 2. Analysis of Inertial Mass Systems for Shake Table Experiments.............. 30

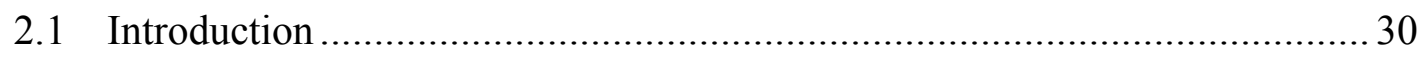

2.2 Inertia Systems for Shake Table Tests........................................................ 31

2.2.1 Inertial Mass Supported by Specimen .................................................... 32

2.2.2 Inertial Mass Supported by External System ......................................... 33

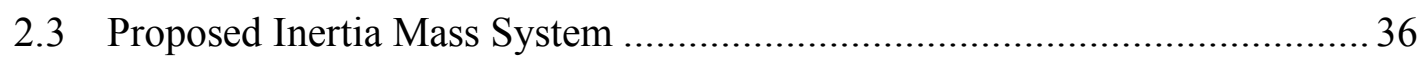

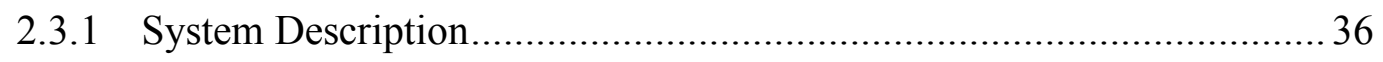

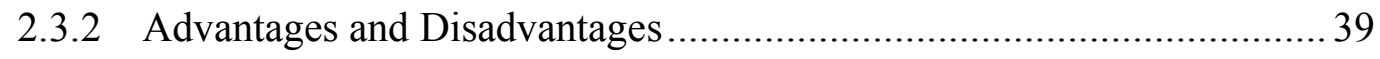

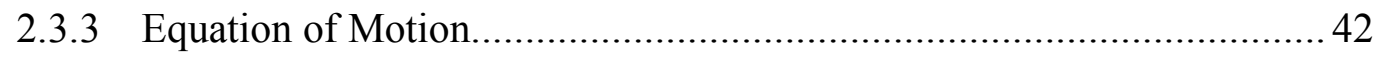


2.3.4 Calculation of Lateral Force .................................................................... 44

2.3.4.1 Using Load Cell .............................................................................. 44

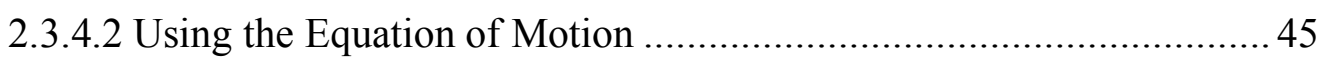

2.3.5 Calculation of P-Delta Force............................................................... 45

2.3.6 Calculation of Damping Coefficient ……………............................... 47

2.4 Numerical Comparison to Existing Inertial Mass Systems ……................... 48

2.4.1 Numerical Modelling of Inertial Mass Systems...................................... 49

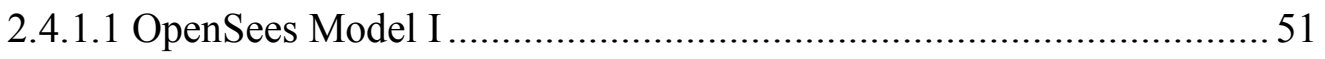

2.4.1.2 OpenSees Model II....................................................................... 55

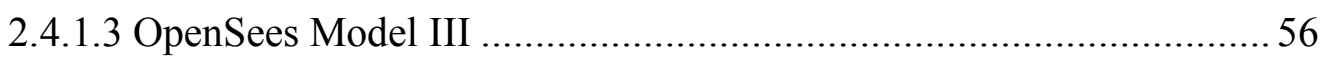

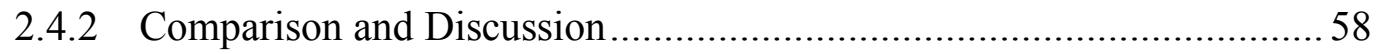

CHAPTER 3. Large-Scale Shake Table Experiments of Substandard RC Bridge Columns

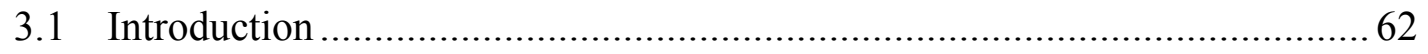

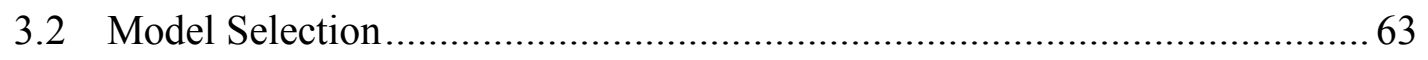

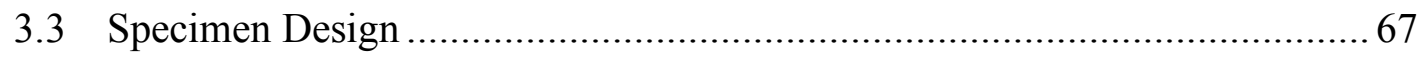

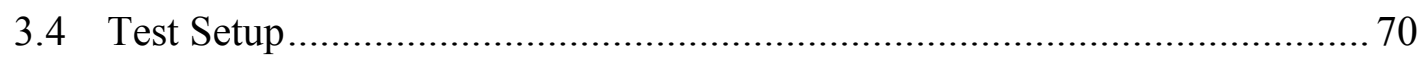

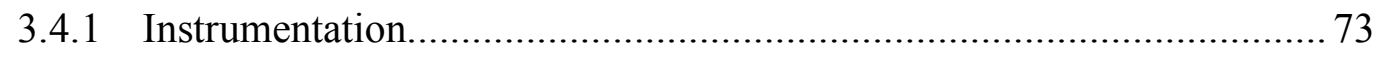

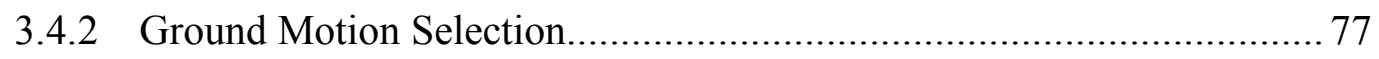

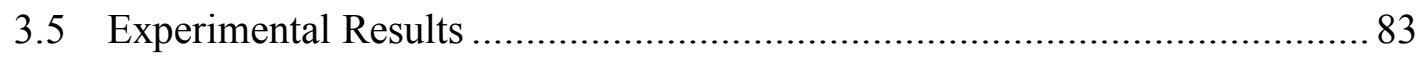

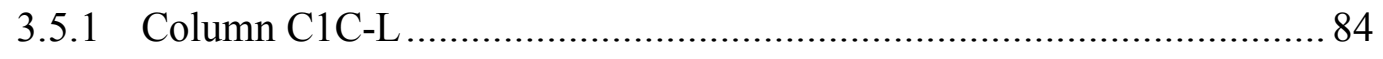

3.5.1.1 Progression of Observed Damage..................................................... 84

viii 


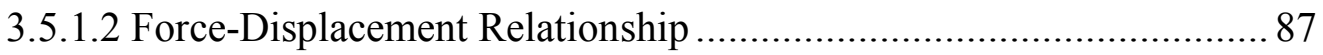

3.5.1.3 Dynamic Properties....................................................................... 92

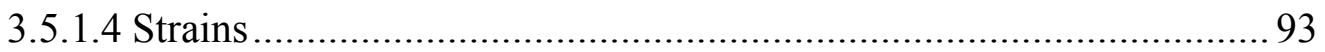

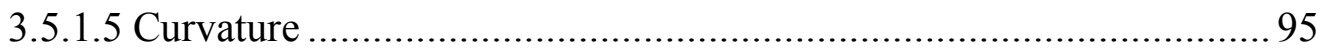

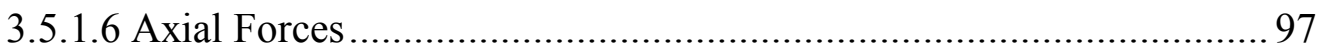

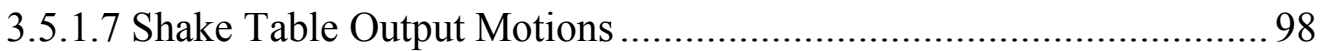

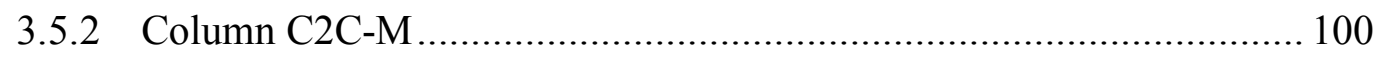

3.5.2.1 Progression of Observed Damage ................................................... 100

3.5.2.2 Force-Displacement Relationship .................................................. 103

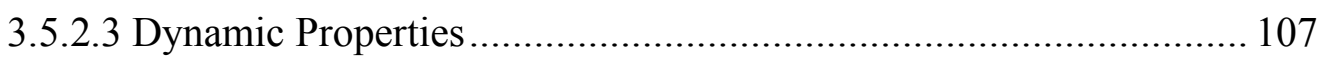

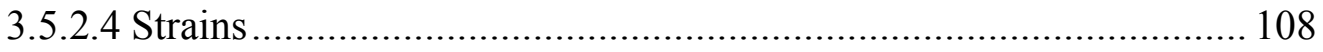

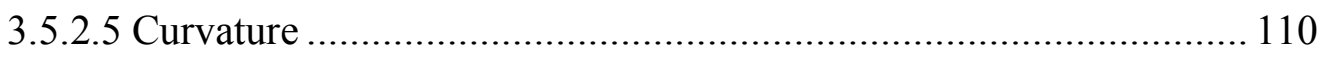

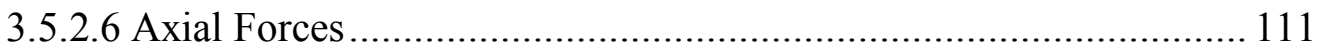

3.5.2.7 Shake Table Output Motions ……………………......................... 112

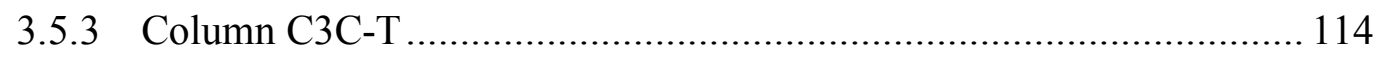

3.5.3.1 Progression of Observed Damage .................................................... 114

3.5.3.2 Force-Displacement Relationship ................................................. 117

3.5.3.3 Dynamic Properties..................................................................... 121

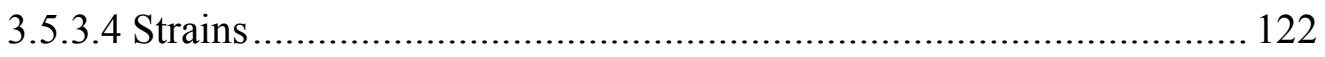

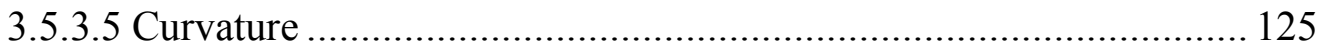

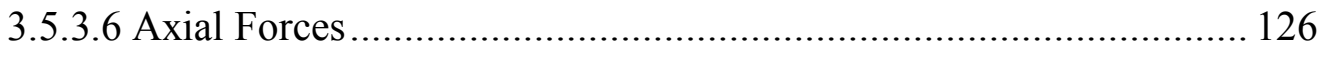

3.5.3.7 Shake Table Output Motions ………………................................ 127 
3.5.4.1 Progression of Observed Damage .......................................... 129

3.5.4.2 Force-Displacement Relationship ........................................ 131

3.5.4.3 Dynamic Properties ............................................................. 134

3.5.4.4 Strains .............................................................................. 135

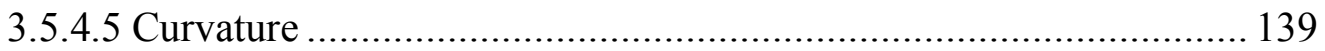

3.5.4.6 Axial Forces .................................................................... 140

3.5.5 Column C5S1-M .................................................................... 141

3.5.5.1 Progression of Observed Damage .......................................... 141

3.5.5.2 Force-Displacement Relationship ...................................... 143

3.5.5.3 Dynamic Properties.......................................................... 146

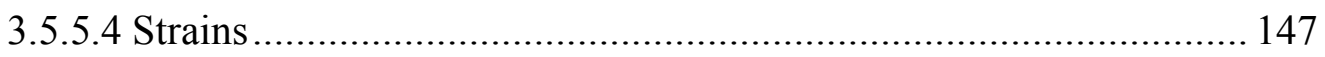

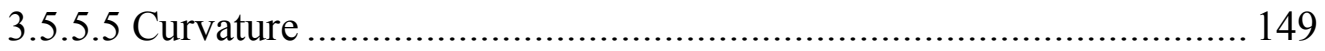

3.5.5.6 Axial Forces ................................................................ 149

3.5.6 Column C6S2-M ................................................................. 150

3.5.6.1 Progression of Observed Damage ........................................... 151

3.5.6.2 Force-Displacement Relationship ........................................ 152

3.5.6.3 Dynamic Properties ...................................................... 154

3.5.6.4 Strains .......................................................................... 155

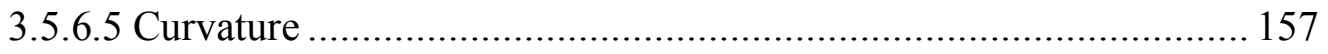

3.5.6.6 Axial Forces ................................................................ 158

3.6 Column Comparisons...................................................................... 159 
3.6.1 Backbone Curve Comparison

3.6.2 Energy Dissipation and Stiffness Degradation

3.6.3 Cumulative Damage .................................................................. 164

3.7 Performance Assessment based on Material Strains ................................. 166

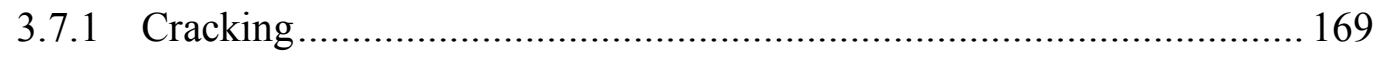

3.7.2 First Yield of Longitudinal Reinforcement.................................... 170

3.7.3 Concrete Cover Spalling ........................................................ 170

3.7.4 Bar Buckling and Core Crushing …........................................ 172

CHAPTER 4. Numerical Investigation of the Effects of Subduction Earthquakes on the

Seismic Performance of Substandard RC Bridge Columns ...................................... 175

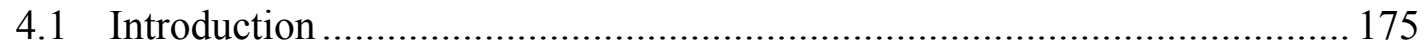

4.2 Numerical Model of Large-Scale Bridge Columns ................................ 176

4.2.1 Results for Specimens with Continuous Reinforcement .................. 181

4.2.2 Results for Specimens with Lap Splice at Hinge Zone ..................... 184

4.3 Numerical Study of a Full-Scale Single RC Bridge Column.................... 188

CHAPTER 5. Preliminary Recommendations of Performance Limits States for RC

Bridge Columns Including the Effects of Subduction Earthquakes .......................... 193

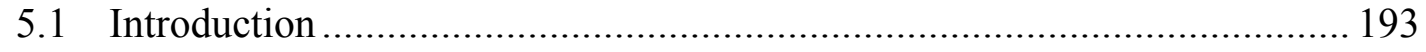

5.2 Performance-Based Seismic Design .................................................. 194

5.3 Seismic Fragility Analysis ............................................................ 196

5.4 Fragility Analysis for Full-Scale RC Bridge Column ............................ 198

5.4.1 Selected Earthquake Ground Motions ......................................... 199 


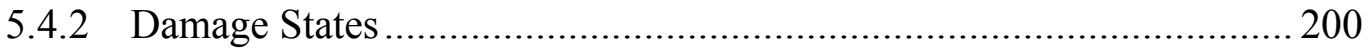

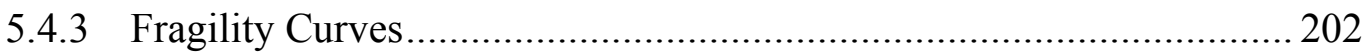

5.5 Strain Limit Sates Recommendations ......................................................... 206

CHAPTER 6. Conclusions and Recommendation for Further Research........................ 208

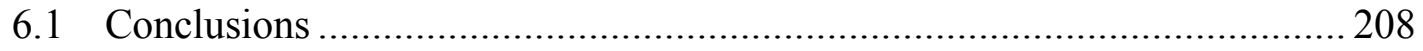

6.1.1 Recommendations for Further Research............................................2 213

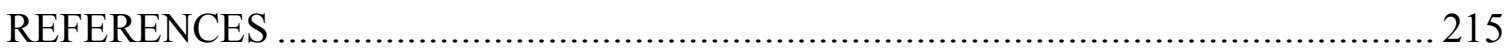

APPENDIX. Aditional Results from Numerical Model Calibration............................ 230 


\section{LIST OF TABLES}

Table 2.1 Masses and dynamic weights of the IMS .................................................... 44

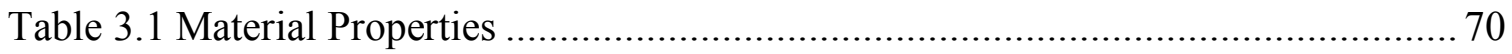

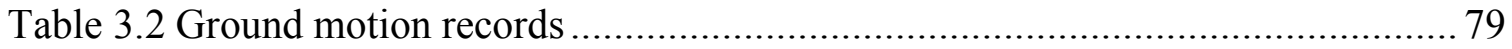

Table 3.3 Scale factors for the selected ground motions............................................. 80

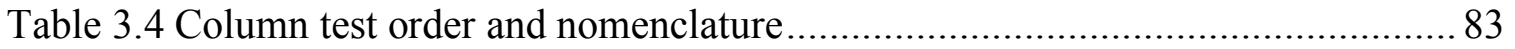

Table 3.5 Summary of the measured performance for C1C-L ..................................... 91

Table 3.6 Measured dynamic properties for C1C-L ……………............................. 93

Table 3.7 Target and achieved peak table responses for Capitola X............................... 99

Table 3.8 Summary of the measured performance for C2C-M ................................... 107

Table 3.9 Measured dynamic properties for C2C-M ............................................. 108

Table 3.10 Target and achieved peak table responses for Curico X.............................. 114

Table 3.11 Summary of the measured performance for C3C-T …............................... 121

Table 3.12 Measured dynamic properties for C3C-T ……......................................... 122

Table 3.13 Target and achieved peak table responses for Iwaki Y .............................. 128

Table 3.14 Summary of the measured performance for C4S1-L................................. 134

Table 3.15 Measured dynamic properties for C4S1-L ............................................ 135

Table 3.16 Summary of the measured performance for C5S1-M ................................ 145

Table 3.17 Measured dynamic properties for C5S1-M.............................................. 147

Table 3.18 Summary of the measured performance for C6S2-M ............................... 154

Table 3.19 Measured dynamic properties for C6S2-M.............................................. 155

Table 3.20 Cumulative Displacement Ductility ......................................................... 166 


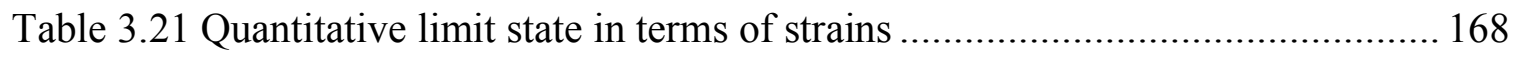

Table 3.22 Component performance levels adapted from Hose \& Seible (1999)......... 169

Table 3.23 Summary of limit state strains for all specimens .................................. 173

Table 3.24 Substandard RC bridge column performance parameters (Limit States) ..... 174

Table 5.1 Summary of the selected ground motions records ................................. 201

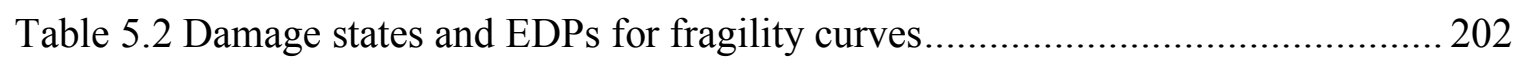

Table 5.3 Fragility curve values for full-scale RC bridge column under subduction

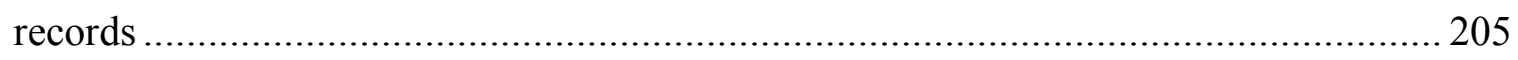

Table 5.4 Fragility curve values for full-scale RC bridge column under crustal records206 


\section{LIST OF FIGURES}

Figure 1.1 Subduction fault zone diagram (USGS, 1999) ..........................................2

Figure 1.2 The Pacific ring of fire, about $90 \%$ of the world's earthquakes occur there

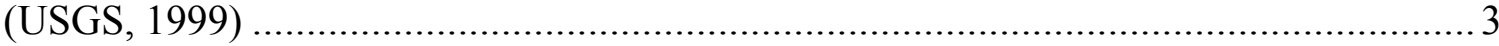

Figure 1.3 Main shock and aftershocks of $\mathrm{M}_{\mathrm{w}} 4$ and larger between 2/27/10 and 3/26/10

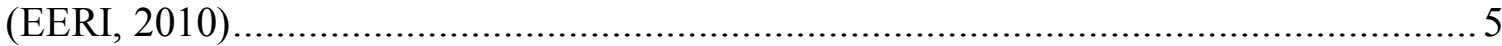

Figure 1.4 Fault zone and damaged region of 2011 Tohoku earthquake (Kawashima \&

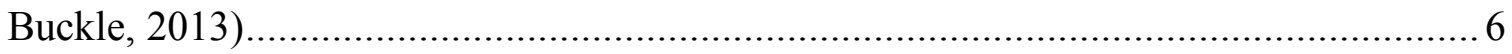

Figure 1.5 Plate tectonics of the Cascadia subduction zone (Leonard et al., 2010) ..........8

Figure 1.6 Support-column failure in Cypress viaduct during the 1989 Loma Prieta

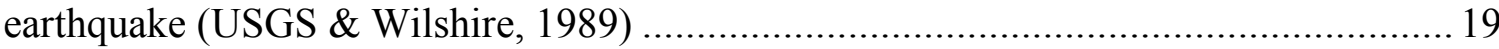

Figure 1.7 Damage in RC columns, 1994 Northridge earthquake (Priestley et al., 1994).

a) Freeway at Fairfax/Washington undercrossing, b) I-118 Mission/Gothic undercrossing

Figure 1.8 Concrete jacketing. a) Concrete overlay (FHWA, 2006), b) Partial height

concrete encasement of a column in Illinois (Poplar Street Complex) ......................... 20

Figure 1.9 Stell jacketing. a) method for circular column, b) Full column steel jacketing used in Tennessee (SR 196 over I-40) ........................................................... 22

Figure 1.10 Composite jacketing. a) continuous fiber composite wrap in Illinois, b) fiber composite wraps applied in strips in Illinois (Poplar Street Complex)......................... 23

Figure 1.11 External prestressing steel retrofit of columns in a bridge in Illinois .......... 24

Figure 1.12 Deck rotation and unseating in skewed bridges (plan view single span) ...... 26 
Figure 1.13 Collapsed bridge due to in-plane rotation and unseating of the deck. a) Miraflores Bridge, b) Lo Echevers Bridge............................................................... 26

Figure 1.14 Damage in RC bridge column due to insufficient shear capacity ................ 27 Figure 1.15 Repair and retrofit for Miraflores bridge (Unjoh, 2012). a) Lateral stopper at abutment, b) lateral beam and lateral stopper at pier ........................................ 28

Figure 1.16 Damage of RC column at section of cut-off of longitudinal reinforcement.. 29 Figure 2.1 UC-Berkeley inertial loading system (Sakai et al., 2005) ........................... 32

Figure 2.2 UC-San Diego inertial loading system (Schoettler et al., 2015)................... 33 Figure 2.3 Unidirectional Mass-rig at UNR (Laplace et al., 1999). a) Actual test setup, b)

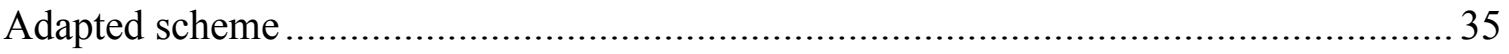

Figure 2.4 Schematic of the IMS part of test setup. a) 3D-view, b) South view............. 38

Figure 2.5 Inertial Mass System (Assembling Process) ........................................ 39

Figure 2.6 IMS reaching maximum displacement limit under specimen collapse .......... 41

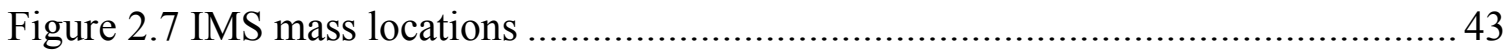

Figure 2.8 P-Delta effect due to axial load...................................................... 47

Figure 2.9 Cross section of RC column model...................................................... 50

Figure 2.10 Time histories for Curico X record. a) Acceleration, b) Displacement.........51

Figure 2.11 OpenSees Numerical Model I for the proposed IMS ...............................52

Figure 2.12 Steel fiber stress-strain relations ....................................................... 55

Figure 2.13 Concrete fiber stress-strain relations ................................................. 55

Figure 2.14 OpenSees Numerical Model II......................................................... 56

Figure 2.15 OpenSees Numerical Model III ........................................................ 58

$\mathrm{xvi}$ 
Figure 2.16 Column displacement history comparison........................................ 59

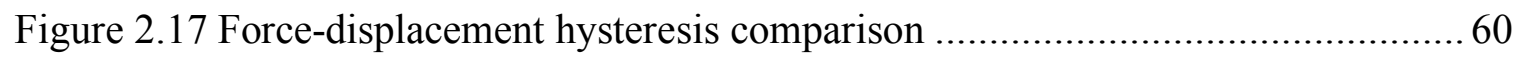

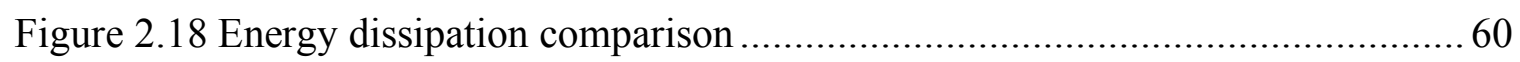

Figure 3.1 Representative multi-column RC bridge bent (Bazaez, 2017) ...................... 64

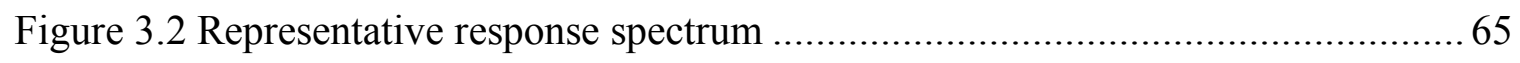

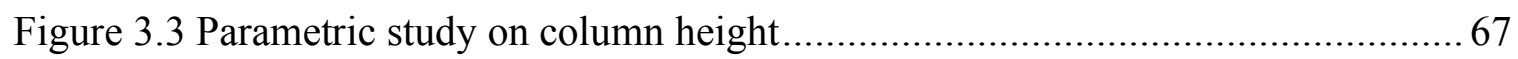

Figure 3.4 Specimens Details. a) Continuous reinforcement, b) Lap-spliced .................. 68

Figure 3.5 Construction sequence for RC bridge columns .................................... 69

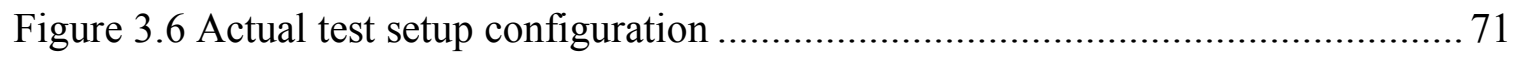

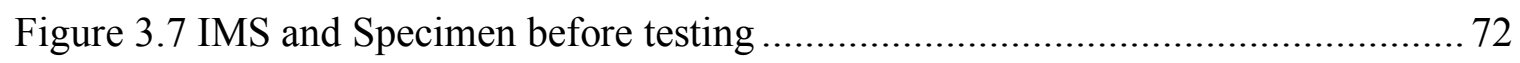

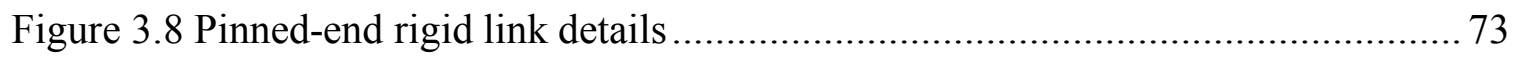

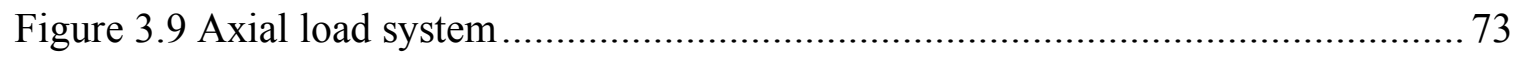

Figure 3.10 Test Setup and Instrumentation...................................................... 74

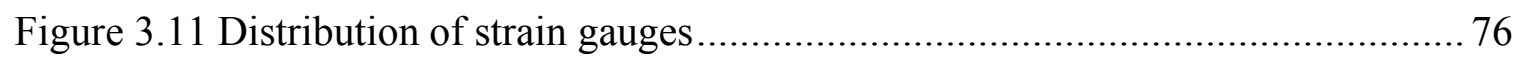

Figure 3.12 Installation of strain gauges. a) Strain gauges on longitudinal reinforcement and hoop, b) Strain gauges on longitudinal reinforcement and dowel........................ 76

Figure 3.13 a) LVDTs layout and one go-pro, b) Accelerometer on column head.......... 77

Figure 3.14 Pre-test force displacement relationships for selected motions a) Amplitude

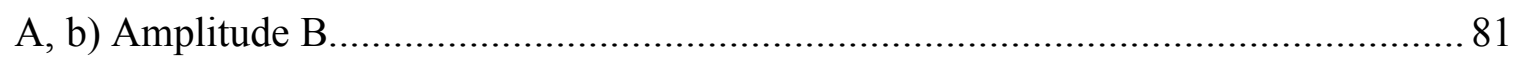

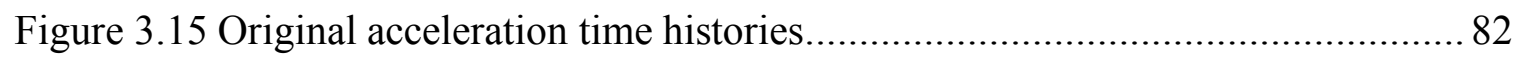

Figure 3.16 Elastic Response Spectra (5\% damping) for amplitude A....................... 82

xvii 
Figure 3.17 Damage state of column C1C-L after applying motion amplitude A. a) North view, b) south-east view 85

Figure 3.18 Damage state of column C1C-L after applying motion amplitude B. a) North view, b) south-east view 86

Figure 3.19 Damage state of column C1C-L after applying motion amplitude C. a) South-

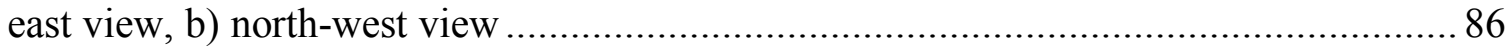

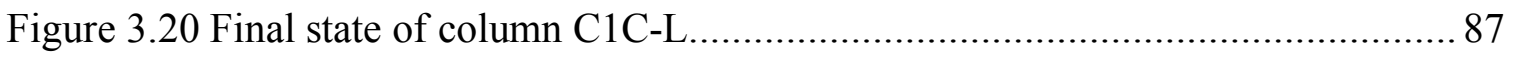

Figure 3.21 Hysteretic response for column C1C-L during motion amplitude A ........... 88

Figure 3.22 Hysteretic response for column C1C-L during motion amplitude B ........... 89

Figure 3.23 Hysteretic response for column C1C-L during motion amplitude C ...........90

Figure 3.24 Column displacement history for column C1C-L ............................... 90

Figure 3.25 Steel strain history for strain gauge N-1-L2 for column C1C-L during motion

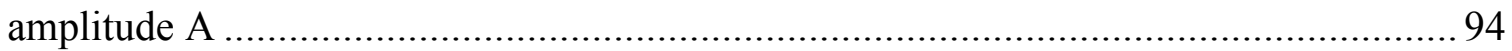

Figure 3.26 Steel strain history for strain gauge S-3-L1 for column C1C-L during motion

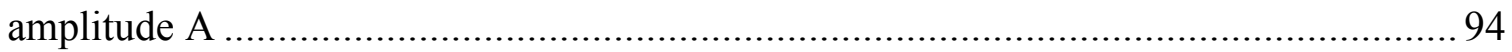
Figure 3.27 Steel strain history for strain gauge N-3-L2 for column C1C-L during motion amplitude B 94 Figure 3.28 Steel strain history for strain gauge S-3-L1 for column C1C-L during motion amplitude B 95

Figure 3.29 Peak steel strain profile for column C1C-L. a) Amplitude A, b) Amplitude B

Figure 3.30 Average curvature profiles in column C1C-L 96 
Figure 3.31 Axial force history for column C1C-L

Figure 3.32 Shake table performance for Capitola X. a) Acceleration time histories, b)

$5 \%$ damped elastic response spectra

Figure 3.33 Damage state of column C2C-M after applying motion amplitude A. a) North view, b) south-east view

Figure 3.34 Damage state of column C2C-M after applying motion amplitude B. a)

North-east view, b) east view

Figure 3.35 Damage state of column C2C-M after applying motion amplitude C. a)

North-east view, b) south-east view

Figure 3.36 Final state of column C2C-M 103

Figure 3.37 Hysteretic response for column C2C-M during motion amplitude A 104

Figure 3.38 Hysteretic response for column C2C-M during motion amplitude B 104

Figure 3.39 Hysteretic response for column C2C-M during motion amplitude $\mathrm{C}$. 106

Figure 3.40 Column displacement history for column C2C-M. 106

Figure 3.41 Steel strain history for strain gauge N-1-L2 for column C2C-M during motion amplitude $\mathrm{A}$

Figure 3.42 Steel strain history for strain gauge S-3-L1 for column C2C-M during motion amplitude A

Figure 3.43 Steel strain history for strain gauge N-3-L2 for column C2C-M during motion amplitude $\mathrm{B}$

Figure 3.44 Steel strain history for strain gauge S-3-L1 for column C2C-M during motion amplitude B. 110

xix 
Figure 3.45 Peak steel strain profile for column C2C-M. a) Amplitude A, b) Amplitude B

Figure 3.46 Average curvature profiles in column C2C-M ................................... 111

Figure 3.47 Axial force history for column C2C-M .............................................. 112

Figure 3.48 Shake table performance for Curico X. a) Acceleration time histories, b) 5\%

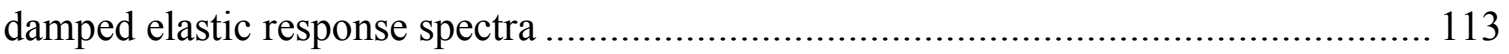

Figure 3.49 Damage state of column C3C-T after applying motion amplitude A. a) North view, b) south-east view 115

Figure 3.50 Damage state of column C3C-T after applying motion amplitude B. a) Northeast view, b) south-west view 116

Figure 3.51 Damage state of column C3C-T after applying motion amplitude C. a) Southeast view, b) east view 116

Figure 3.52 Final state of column C3C-T. 117

Figure 3.53 Hysteretic response for column C3C-T during motion amplitude A ......... 118 Figure 3.54 Hysteretic response for column C3C-T during motion amplitude B ......... 118 Figure 3.55 Hysteretic response for column C3C-T during motion amplitude C ......... 119 Figure 3.56 Column displacement history for column C3C-T .............................. 120 Figure 3.57 Steel strain history for strain gauge N-2-L1 for column C3C-T during motion amplitude A 123 Figure 3.58 Steel strain history for strain gauge S-3-L1 for column C3C-T during motion amplitude A 123 
Figure 3.59 Steel strain history for strain gauge N-1-L1 for column C3C-T during motion amplitude B

Figure 3.60 Steel strain history for strain gauge S-3-L1 for column C3C-T during motion amplitude B 124

Figure 3.61 Peak steel strain profile for column C3C-T. a) Amplitude A, b) Amplitude B

Figure 3.62 Average curvature profiles in column C3C-T .................................... 125

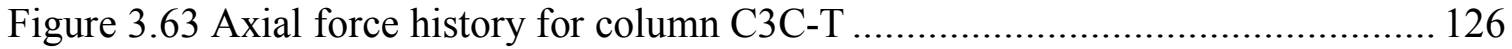

Figure 3.64 Shake table performance for Iwaki Y. a) Acceleration time histories, b) 5\% damped elastic response spectra 128

Figure 3.65 Damage state of column C4S1-L after applying motion amplitude A. a)

North-east view, b) south-east view

Figure 3.66 Damage state of column C4S1-L after applying motion amplitude B. a) North view, b) south-west view

Figure 3.67 Final state of column C4S1-L

Figure 3.68 Hysteretic response for column C4S1-L during motion amplitude A 132

Figure 3.69 Hysteretic response for column C4S1-L during motion amplitude B

Figure 3.70 Column displacement history for column C4S1-L

Figure 3.71 Steel strain history for strain gauge N-D1-L1 for column C4S1-L during motion amplitude A

Figure 3.72 Steel strain history for strain gauge S-D3-L1 for column C4S1-L during motion amplitude $\mathrm{A}$ 
Figure 3.73 Steel strain history for strain gauge N-D1-L1 for column C4S1-L during motion amplitude $\mathrm{B}$

Figure 3.74 Steel strain history for strain gauge S-D3-L1 for column C4S1-L during motion amplitude $\mathrm{B}$

Figure 3.75 Peak steel strain profile for column C4S1-L. a) Amplitude A, b) Amplitude B

Figure 3.76 Average curvature profiles in column C4S1-L .................................... 140

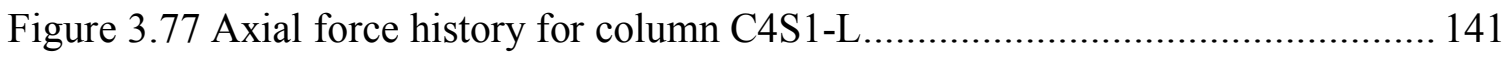

Figure 3.78 Damage state of column C5S1-M after applying motion amplitude A. a)

North-west view, b) south-east view................................................................ 142

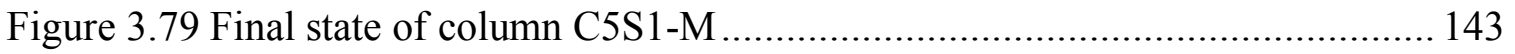

Figure 3.80 Hysteretic response for column C5S1-M during motion amplitude A ....... 144

Figure 3.81 Column displacement history for column C5S1-M ............................. 145

Figure 3.82 Steel strain history for strain gauge N-D1-L2 for Column C5S1-M during

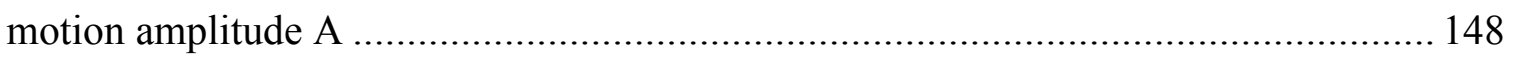

Figure 3.83 Steel strain history for strain gauge S-D3-L1 for Column C5S1-M during

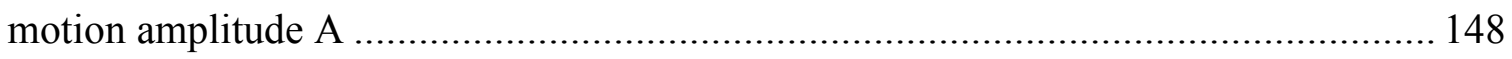

Figure 3.84 Peak steel strain profile for Column C5S1-M..................................... 148

Figure 3.85 Average curvature profiles in column C5S1-M ................................. 149

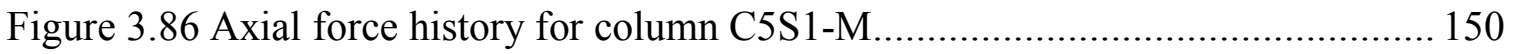

Figure 3.87 Damage state of column C6S2-M after applying motion amplitude A. a)

North-east view, b) south-east view 
Figure 3.88 Final state of column C6S2-M

Figure 3.89 Hysteretic response for column C6S2-M during motion amplitude A ....... 153

Figure 3.90 Column displacement history for column C6S2-M ............................. 154

Figure 3.91 Steel strain history for strain gauge N-1-L2 for column C6S2-M during

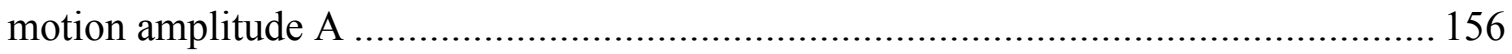

Figure 3.92 Steel strain history for strain gauge S-3-L1 for column C6S2-M during

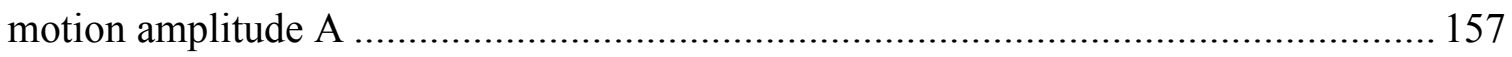

Figure 3.93 Peak steel strain profile for column C6S2-M ................................... 157

Figure 3.94 Average curvature profiles in column C6S2-M .................................. 158

Figure 3.95 Axial force history for column C6S2-M............................................ 159

Figure 3.96 Backbone curves comparison for all specimens. a) Amplitude A, b)

Amplitude B

Figure 3.97 Lateral stiffness degradation after each ground motion amplitude for all specimens. a) Amplitude A, b) Amplitude B

Figure 3.98 Energy dissipation comparison for all specimens. a) Amplitude A, b)

Amplitude B

Figure 4.1 Hysteresis curves comparison for all motion amplitudes. a) C1C-L, b) C2C-M

Figure 4.2 Steel strain history comparison for Column C1C-L-Bar \#3. a) Amp.A, b)

Amp.B

Figure 4.3 Steel strain history comparison for Column C2C-L - Bar \#1. a) Amp.A, b)

Amp.B 
Figure 4.4 Steel strain history comparison for Column C2C-L - Bar \#3. a) Amp.A, b)

Amp.B

Figure 4.5 Hysteresis curves comparison for lap-spliced specimen. a) C4S1-L during Amp.A, b) C4S1-L during Amp.B, c) C5S1-M during Amp.A, d) C6S2-M during Amp.A

Figure 4.6 Steel strain history comparison for Column C4S1-L in Amp.A. a) Bar \#1, b)

Bar \#3

Figure 4.7 Steel strain history comparison for Column C5S1-M in Amp.A. a) Bar \#1, b)

Bar \#3

Figure 4.8 Steel strain history comparison for Column C6S2-M in Amp.A. a) Bar \#1, b)

Bar \#3

Figure 4.9 Results of NLTHA for the full-scale single bridge column

Figure 4.10 Steel strain histories for the full-scale single bridge column.

Figure 5.1 Visualization of Performance Based Seismic Design (Adapted from (NCHRP, 2013)) 196

Figure 5.2 Schematic representation of the NLTHA procedure used for Fragility Analysis 198

Figure 5.3 Elastic response spectra of the ground motions used in this study (5\% damping ratio). a) Subduction set, b) Crustal set 200

Figure 5.4 Peak tensile steel strain fragility curves. a) Moderate, b) Collapse 205

Figure 5.5 Peak compressive concrete strain fragility curves. a) Moderate, b) Collapse205 


\section{CHAPTER 1. Introduction and Previous Research}

\subsection{Research Motivation}

The recent occurrence of highly damaging, long-duration subduction earthquakes in Chile (Maule, 2010) and Japan (Tohoku, 2011), has highlighted the necessity of studying the effects of subduction-zone ground motion on structural performance. These events have exposed the vulnerability of reinforced concrete bridges to the increasing duration of ground shaking. Even more interesting is the possibility of occurrence of another large-magnitude, long-duration subduction earthquake along the Cascadia subduction zone in the Pacific Northwest coast of the United States (Heaton \& Kanamori, 1984). Given the uncertainty of how bridges will perform under long-duration megathrust events, have raised researchers' interest to study this topic. Firstly, current seismic design codes do not explicitly consider the effects subduction-zone events, and they just rely on a single acceleration design value. This design methodology is the result of the scarcity of available data of large subduction zone earthquakes and the disagreement in the research community on their effects on structural performance. However, the aforementioned seismic events have provided an extensive database of long-duration subduction-zone records to study this topic better. Secondly, the extent of damage is not directly considered in current design codes, and little attention is paid in satisfy specified performance levels. To overcome this situation, some Departments of Transportation in the United States have adopted the performance-based seismic design criteria (e.g., (ODOT, 2015)), where a structural performance level is a specific damage state and is 
quantified using engineering limit states. Thus, evaluating performance limit states can be a suitable approach to contrast structural damage effect from subduction zone earthquakes.

\subsection{Subduction-Zone Megathrust Earthquakes}

Earthquakes are caused by rupture and slippage along geological faults as it releases strain that has accumulated over long a long time. As a result, seismic waves are emitted outward from the source of the earthquake. Among the three types of faults, normal, thrust (reverse) and strike-slip, megathrust earthquakes are the planet's most powerful, and are the only source zones that can produce earthquakes greater than $\mathrm{M}_{\mathrm{w}}=$ 8.5. Megathrust earthquakes arise at subduction zones at destructive convergent plate boundaries, where one tectonic plate is thrust beneath another (Figure 1.1). Besides their large magnitude, subduction earthquakes are characterized by the large rupture area and large energy released during the event.

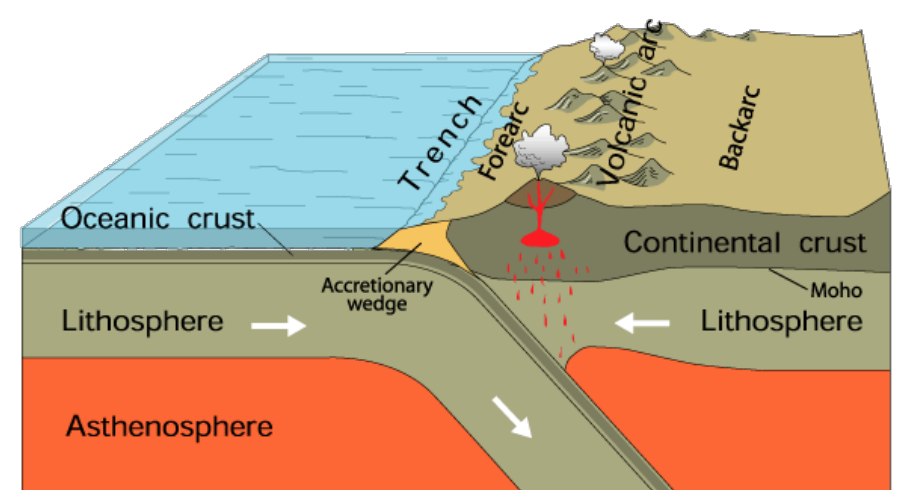

Figure 1.1 Subduction fault zone diagram (USGS, 1999)

Another important feature of a megathrust earthquake is their long-duration. It is widely known that earthquake duration increases with increasing earthquake magnitude. Therefore, when an earthquake is of larger magnitude the dimensions of fault rupture is 
larger. Since the dislocation velocity does not change significantly with magnitude, the duration of fault rupture, which is closely related to the duration of the earthquake record, generally increases with increasing earthquake magnitude. Another aspect that increases the duration of a seismic event is the increasing distance from the recording site to the zone of energy release of the causative earthquake. Earthquake duration also is greater at soils sites than at rock sites. Earthquake records on soils sites have an additional long period portion not seen in rock records. As example, the largest recorded megathrust earthquake in the world was the $1960 \mathrm{M}_{\mathrm{w}}=9.5$ Valdivia earthquake. The earthquake's rupture zone was $800 \mathrm{~km}$ and lasted approximately 10 minutes. Now, recent devastating long-duration subduction-zone megathrust earthquakes are the $2004 \mathrm{M}_{\mathrm{w}}=9.0$ SumatraAndaman earthquake, the $2005 \mathrm{M}_{\mathrm{w}}=8.7$ Nias-Simeulue earthquake, the $2010 \mathrm{M}_{\mathrm{w}}=8.8$ Maule earthquake, and the $2011 \mathrm{M}_{\mathrm{w}}=9.0$ Tohoku earthquake, and all occurred in the pacific ring of fire (Figure 1.2).

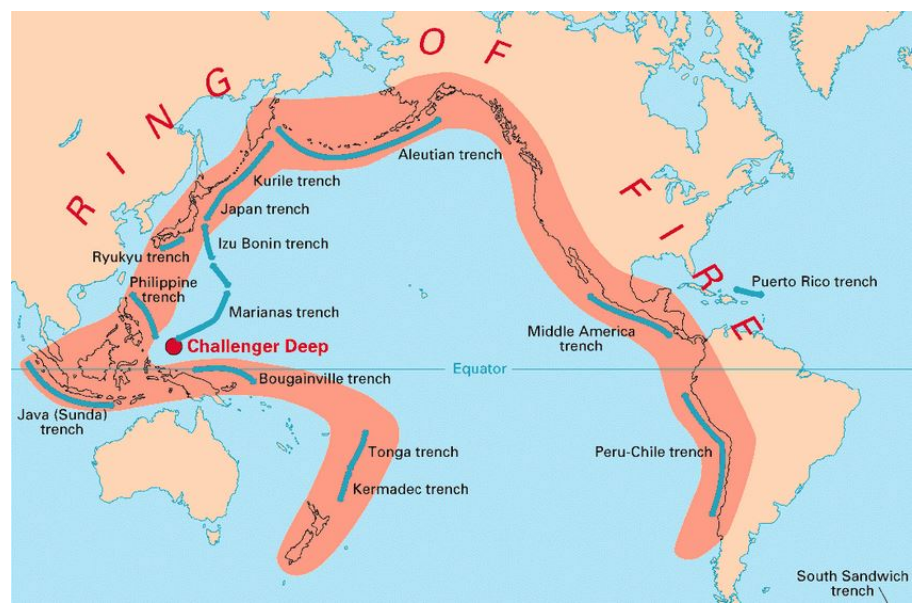

Figure 1.2 The Pacific ring of fire, about $90 \%$ of the world's earthquakes occur there (USGS, 1999) 
One of the causes of the resulting damages due to earthquake duration is associated with soil liquefaction, and the significant soil displacements that are characteristic of this phenomenon, in which loose sand and silt that is saturated with water can behave like a liquid when shaken by an earthquake. The 2010 Chile Earthquake and the 2011 Tohoku Earthquake have repeated the catastrophic experience left by large earthquakes in other highly seismic areas, including the occurrence of soil liquefaction at several sites. The effects of soil liquefaction on the built environment can be extremely damaging. Bridges whose foundations bear directly on sand which liquefies will experience a sudden loss of support, which will result in drastic and irregular settlement of the bridges causing structural damage, including cracking of foundations and damage to the bridge structure, or leaving the structure unserviceable, even without structural damage. Also, Bridges and large buildings constructed on pile foundations may lose support from the adjacent soil and buckle, or come to rest at a tilt. Thus, soil liquefaction induced by earthquake shaking is a major contributor to urban seismic risk.

\subsubsection{Chile Subduction Zone}

Chile is located in one of the most active subduction zones in the planet. The occurrence of earthquakes in the Chilean territory is explained by the underthrusting movement of the Nazca plate underneath the South American plate. Most of the large earthquakes, moment magnitude 8 or greater, have occurred on the interface between both plates, where the most prominent example is the $1960 \mathrm{M}_{\mathrm{w}}=9.5$ Valdivia earthquake, which is the world's largest and powerful earthquake ever recorded. Additionally, six seismic events of magnitude greater than $\mathrm{M}_{\mathrm{w}}=8.0$ have occurred in the 
last 10 years, including the $2010 \mathrm{M}_{\mathrm{w}}=8.8$ Maule, the $2014 \mathrm{M}_{\mathrm{w}}=8.2$ Iquique, and the $2015 \mathrm{M}_{\mathrm{w}}=8.3$ Illapel earthquakes.

On February 27, the 2010 Maule earthquake struck off the coast of central Chile. It produced an intense shaking lasting for about three minutes and ruptures $500 \mathrm{~km}$ of the Chile subduction margin. Figure 1.3 shows the location of the main shock and aftershocks associated relative to major cities (EERI, 2010). In the region of strongest ground shaking, ground acceleration exceeded $0.05 \mathrm{~g}$ for over $120 \mathrm{sec}$. Coastal locations were affected by both, ground shaking and tsunami. This long-duration seismic event is one of the largest magnitude events to have produced strong motion recordings worldwide, contributing with important accelerograms dataset for studying the effects of longduration subduction earthquakes for different site soil conditions.

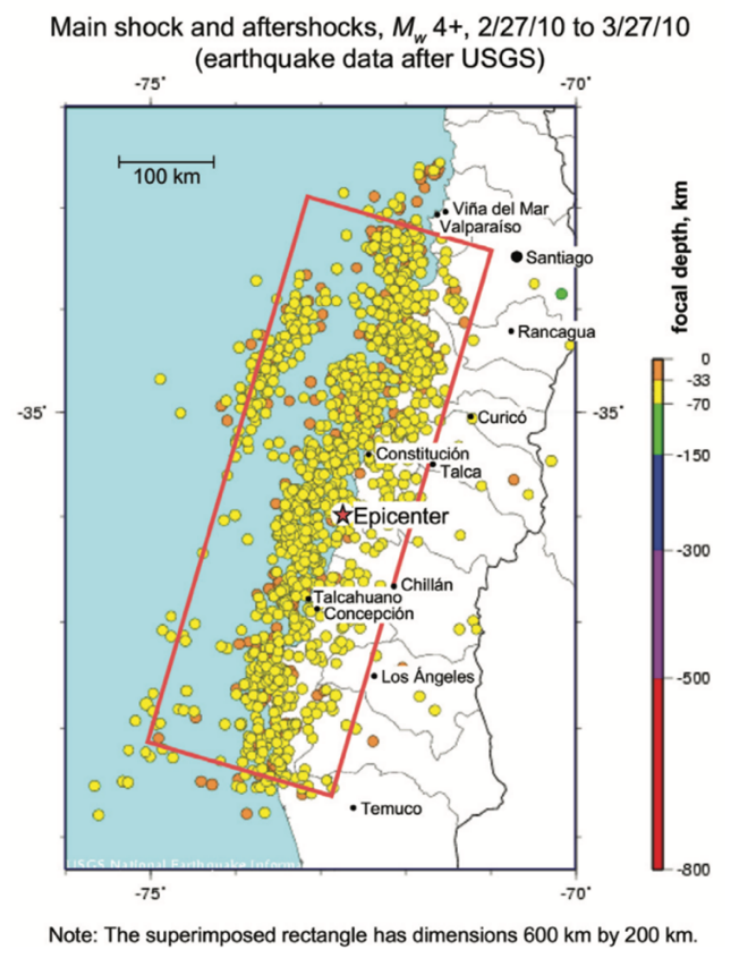

Figure 1.3 Main shock and aftershocks of $\mathrm{M}_{\mathrm{w}} 4$ and larger between 2/27/10 and 3/26/10 (EERI, 2010) 


\subsubsection{Japan Subduction Zone}

The Japanese Islands are located in a complex subduction zone created by four major convergent boundaries crustal plates. The subduction process is a result of the subducting movement of the Pacific plate beneath the Okhotsk plate, and the northwestward and descending movement of the Philippine Sea plate beneath the Eurasian Plate. Historically, an important number of moment magnitude $8+$ earthquakes have occurred on the Japan Trench megathrust (Simons et al., 2011). The most notable and destructive seismic event on this subduction zone was the $2011 \mathrm{Mw}=9.0$ Tohoku earthquake, an undersea megathrust earthquake that struck off the coast of Japan on March 11, 2011. This long-duration earthquake caused a fault rupture of $500 \mathrm{~km}$ long by $200 \mathrm{~km}$ wide, and lasted approximately 3 minutes. Figure 1.4 shows the fault zone and damaged region of the 2011 Tohoku earthquake. It is the most powerful earthquake ever recorded in Japan, and the fourth most powerful earthquake in the world. As the 2010 Maule earthquake, this event also triggered a large and damaging tsunami.

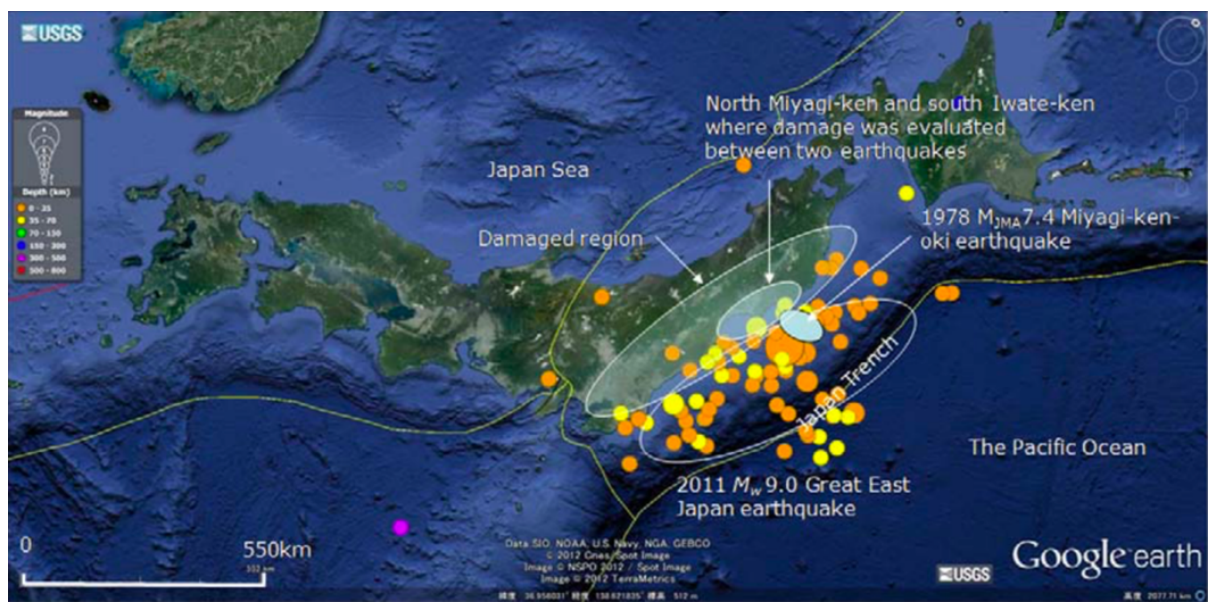

Figure 1.4 Fault zone and damaged region of 2011 Tohoku earthquake (Kawashima \& Buckle, 2013) 


\subsubsection{Cascadia Subduction Zone}

The Cascadia Subduction Zone (CSZ) "megathrust" fault is a 1,000 km long dipping fault that stretches from northern Vancouver Island to Northern California. This convergent plate boundary is created by the movement of the Juan de Fuca plate beneath the North American plate, and it is thought to be capable of producing large subduction earthquakes in the Pacific Northwest (Figure 1.5). Due to the great length of the fault, the Cascadia Subduction Zone is capable of producing very largemagnitude long-duration events if rupture occurs along its entire length. Geological evidence has shown that giant earthquakes occurring near the coast have occurred in the past (Goldfinger et al., 2008). Among these past events, the $M_{w}=9.0$ earthquake of 1700, caused a large tsunami that struck the coast of Japan (Atwater et al., 2005). Historical evidence combined with comparisons of the Cascadia fault to other subduction zones has led geologists to conclude the high likelihood of occurrence of a megathrust earthquake in the Cascadia subduction zone in the future (Heaton \& Kanamori, 1984).

Research has shown that the Cascadia subduction zone is similar in many aspects to other subduction zones in the world that have experienced large earthquakes (Marsh \& Gianotti, 1995). It has a similar convergence rate between plates to that of southwestern Japan. The age of the subducting crust is close to that of the southwestern Japan and southern Chile, all of which are about 20 million years old. Furthermore, the depth of recorded seismic action in the CSZ is shallow, less than 90 kilometers, which is similar to the subduction areas in Chile and Alaska that also have shallow active zones (Heaton \& 
Kanamori, 1984). The lack of recent large subduction earthquakes under this thrusting fault has raised concern regarding the subduction process in the Cascadia Zone. If the subduction zone ruptures along its entirely $1000 \mathrm{~km}$ length, earthquakes may be as large as the 1960 Valdivia earthquake or the 1964 Alaska earthquake (Heaton \& Hartzell, 1986).

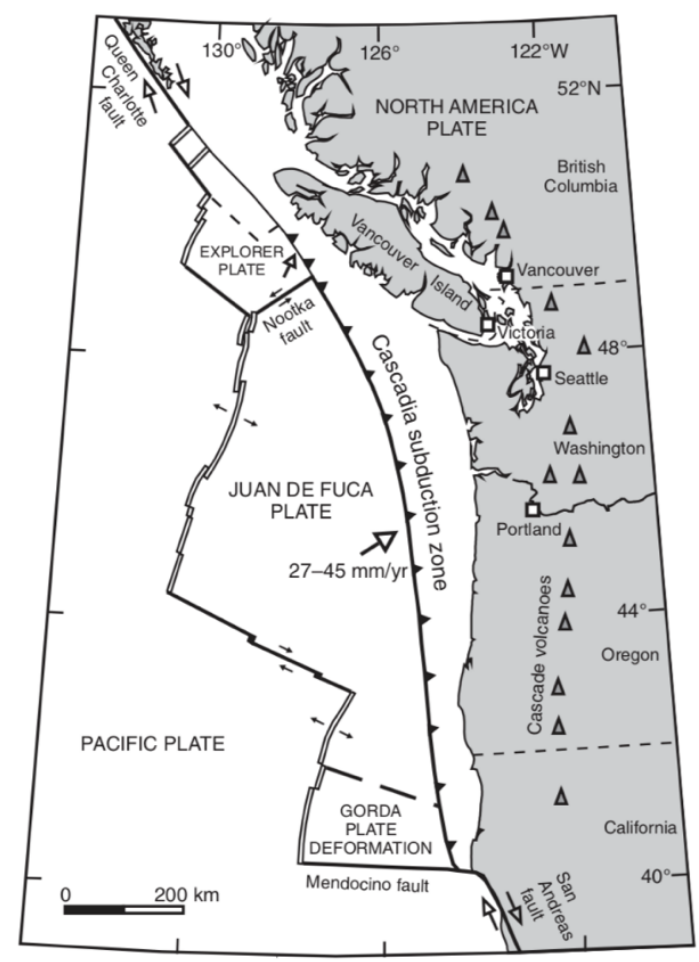

Figure 1.5 Plate tectonics of the Cascadia subduction zone (Leonard et al., 2010)

\subsection{Previous Research Including Effect of Subduction Zone Earthquakes}

Recent devastating, long-duration megathrust subduction-zone earthquakes occurred in the world emphasize the potential of having a severe longer duration larger magnitude ground shaking than past events at some sites. Such earthquake magnitude can produce a large fault rupture, and therefore, a long duration event (Dobry et al., 1978), (Marsh \& Gianotti, 1995). Further, the possibility of the occurrence of another large 
magnitude long-duration subduction zone earthquake along the northwest coast of North America, which lies near the Cascadia Subduction Zone (CSZ), has raised the necessity of more thorough understanding of the differences in structural performance resulting from long-duration large-magnitude subduction zone earthquakes. The effect of earthquake duration on structural damage has been of significant interest in the research community, although the findings have been inconclusive and controversial. Some researchers have concluded that there is a high correlation between strong motion duration and structural damage, whereas others have found no relation (Hancock \& Bommer, 2006). Numerical studies using peak response (e.g., maximum displacement or drift) as a measure of damage have found no correlation between ground motion duration and increasing damage (Iervolino et al., 2006; Hancock \& Boomer, 2007). In contrast, numerical studies using cumulative damage measures or energy measures, have found a high correlation between duration and damage (Hancock \& Boomer, 2007; (Özer et al., 2012). Then, structural systems or components become increasingly vulnerable given the increasing number of cyclic reversals caused by long duration motions (Bertero et al., 1977; Marsh \& Gianotti, 1995). Thus, the occurrence of a long-duration, large-magnitude event, like those mentioned earlier, has the potential of significantly increasing the number of inelastic incursions and therefore, accumulate more damage (Raghunandan \& Leil, 2013; Chandramohan et al., 2013). However, there is still a lack of experimental evidence that can verify previous findings. This section presents some of the previous studies that have investigated the effects of subduction zone earthquakes, specifically earthquake duration, on structural performance. 


\subsubsection{Marsh and Gianotti (1995)}

A numerical research conducted by Marsh and Gianotti (1995) investigated the effects of Cascadia Subduction Zone effects on inelastic structural response. SDOFS systems with either bilinear or degrading stiffness hysteretic models were subjected to long-duration artificial acceleration records. The results of this study showed that the damage caused by long-duration, large magnitude CSZ earthquake would be higher than the damage expected for current to that date code acceleration records. It was found that damage based upon dissipated inelastic energy is directly related to the duration of the record. The structures subjected to long-duration CZS records experienced considerable inelastic action, and therefore, damage accumulated as a result of repeated cycles but the displacement cycle demands were not of high values. Nevertheless, they pointed out that total displacement demands produced by CSZ records were not excessive. The authors also stated that many uncertainties and assumption made in modelling both the artificial ground motions and response behavior might affect the results obtained and conclusions made.

\subsubsection{Kunnath et al. (1997)}

Kunnath et al. (1997) experimentally studied the cumulative seismic damage in twelve flexural-dominated circular reinforced concrete bridge columns. The specimens were tested in two phases, one using standard cyclic loads and the other one using analytically predicted displacement amplitudes that represent bridge column response to typical earthquakes. The authors concluded that the use of random cycles is a more reliable way for testing structures subjected to low-cycle fatigue damage and provide a 
better means in studying the cumulative damage than standard cyclic loads. The test results shown two failure modes, low cycle fatigue of the longitudinal reinforcing bars and confinement failure due to rupture of the confinement spirals. It was also found that under predominant low amplitude cycles, confining spiral will fail prior to low-cycle fatigue of longitudinal bars, however, for predominant high amplitude inelastic cycles, rupture of longitudinal bars will occur before confinement failure. Finally, the study showed the energy capacity of a member at failure is strongly history dependent.

\subsubsection{Thompson (2004)}

Thompson (2004) numerically evaluated the response of RC bridges to longduration earthquakes. For that purpose, two highway bridges with poorly confined columns were modeled with finite element spine models. The analysis performed incorporated short and long-duration records, and bridge response and damage were evaluated. The results from the study showed that neither the short or long-duration events will cause significant damage in the bridge columns. Even though the records used have many more loading cycles, they were not at levels that cause yielding, and hence, caused minor damage to the bridge columns. It is important to mention that the records used in the study classified the duration of shaking using bracketed duration, which overestimate the duration of the motion as compared to significant duration. Therefore, when comparing the records used by the author, they are short as compared to records from the 2010 Maule earthquake or the 2011 Tohoku earthquake. 


\subsubsection{Stapleton et al. (2005)}

In this work, the performance of substandard reinforced concrete columns under long-duration subduction zone earthquakes was studied. With that purpose, eight identical circular reinforced concrete columns were tested using different displacement histories that aimed to replicate a severe Cascadia subduction zone event. For that purpose, the loading protocol included one displacement history based on the 1985 Chile subduction zone earthquake, and another one from the 2001 Peru subduction zone earthquake. Results showed that the failure mode of the columns was strongly dependent on the displacement history used in testing. Thus, it is important to choose the right displacement history for testing when studying the effect of long-duration on structural components. However, the authors concluded that lightly confined RC bridge columns built in the 1950s and 1960s in western Washington State will undergo minor damage in the predicted CSZ earthquake.

\subsubsection{Ranf et al. (2006)}

Ranf et al. (2006) evaluated the effects of cyclic loading on the damage accumulation of RC columns. The experimental program comprised six nominally identical lightly confined circular RC bridge columns and subjected to a variety of displacement histories. It was found that the maximum column displacement preceding failure decreased by $35 \%$ when increasing the number of cycles from one to fifteen at the same displacement level. Three damage models were studied and it was found that the Cumulative Plastic Deformation model correlated better for characterizing the effects of cycling on column performance. 


\subsubsection{Ou et al. (2013)}

The seismic behavior under long-duration ground motions of well-detailed reinforced concrete bridge columns was investigated experimentally and analytically. Cyclic loading protocols were developed to represent the number of response cycles expected under long and short duration seismic events. For that purpose, thirty-six long duration and 36 short-duration ground motion were considered in this study and the number of crossing the 5\% PGA level was used to define the duration of the records. Test results showed that columns under a long duration protocol had a similar peak strength but a lower ductility capacity as compared to the short-duration protocol. It was also observed that at $3 \%$ drift, the effects of duration were different. At $3 \%$ drift or smaller, the number of cycles has greater effects on the column stiffness than on column strength, whereas for exceeding that drift, the strength of the column under long duration loading protocol was significantly degraded by longitudinal bar buckling. In the case of shortduration loading protocol buckling of longitudinal reinforcement did not occur until 5\% was reached, and followed by substantial strength degradation.

\subsubsection{Goodnight et al. (2013)}

In this work, the importance and influence of displacement history on performance limit states was investigated. Eight nominally identical well-confined circular RC bridge columns were tested under various unidirectional loading histories. Columns were subjected to conventional laboratory reversed cyclic loading and displacement responses from nonlinear time history analysis of crustal and subduction ground motions. An important conclusion from this work is that the loading history affects the buckling of the longitudinal 
reinforcing steel due to the accumulated strains within the longitudinal and transverse reinforcement.

\subsubsection{Hou and Qu (2015)}

Hou and Qu (2015) investigated the effects of earthquake duration on the ductility and energy dissipation demands on structures. A total of 80 spectrally equivalent ground motion from historical records were used in response history analysis of elastic-perfectly plastic SDOFS. Significant duration based on 5-95\% of the total Arias Intensity was used as duration metric and varied between 6.22 and $145.2 \mathrm{sec}$. One of the outcomes of this study was that long duration ground motions has a significant impact on hysteretic energy dissipation demands but insignificant influence on ductility demands as compared to short duration ground motions.

\subsubsection{Chandramohan et al. (2016)}

This work studied the influence of ground motion duration on the collapse capacity of a five-story steel moment frame building and a reinforced concrete bridge pier. Nonlinear dynamic analyses were performed using two set of long and short duration records from real earthquakes. The 5-75\% significant duration was utilized as duration metric. The results shown that structural collapse capacity is significantly influenced by ground motion duration. The median collapse capacity of the steel building under the long duration set was decreased by a $29 \%$, compared to the short duration one. In the case of the bridge pier, a $17 \%$ reduction in the median collapse capacity was estimated by the long duration set, compared to the short duration one. Additionally, a 
comparison of duration metrics was studied and it revealed the significant duration as a preferred duration metric for structural performance assessment.

\subsubsection{Mohammed (2016)}

The objective of this research was to study the influence of ground motion duration on the collapse capacity of RC bridge columns. Shake table test of five identical, 1/3-scale well-detailed circular columns were performed using long and short duration motion records, based on significant duration. Each motion was incrementally amplified until failure of the specimen was reached. The test results showed that for long duration ground motions, the column's displacement capacity was significantly reduced. The geometric mean of the displacement capacities for the long-duration specimens was $32 \%$ lower than the maximum displacement capacity of the short-duration specimen. Incremental dynamic analysis was performed to the column model calibrated against experimental results. Different sets of spectrally equivalent long and short-duration motions were considered. Comparative collapse analysis showed a reduction on the displacement collapse capacity of long-duration motions of about $25 \%$ compared to short-duration records. The main conclusion of this work is that ground motion duration has a significant effect on the collapse capacity of bridge columns and it is recommended to include the effect in seismic design provision for bridges. As design recommendation, the author propose that for displacement-based design of bridge columns, a $25 \%$ reduction should be applied to the column design displacement capacity for locations where long duration seismic events are expected. 


\subsection{Research Objectives}

The objectives of my doctoral study are as follow:

(i) Experimentally compare and characterize the difference in damage caused by crustal and subduction-zone ground motions.

(ii) Quantify the effects of duration on structural damage to describe performance levels in an effort to evaluate current performance criteria.

(iii) Propose and validate numerical models based on experimental results in an effort to aid designers and researchers in performing nonlinear analysis.

(iv) Develop seismic fragility curves to evaluate damage states for bridge columns.

(v) Recommend whether the effects of subduction megathrust earthquakes should be included in the seismic design and retrofit of bridges.

\subsection{Contribution to the Field}

The main contributions of this research are as follows:

(i) Investigate the performance of substandard reinforced concrete bridge columns subjected to subduction-zone ground motions through large-scale shake table tests.

(ii) Propose and analyze an inertia mass system to provide the necessary representative forces during real time earthquake experiments.

(iii) Develop numerical models of RC bridge columns, calibrated against obtained experimental results, to evaluate the damage states of bridge columns using numerical fragility curves. 
(iv) Propose performance criteria recommendations used in seismic design procedures and retrofit manuals of bridges that include the effect of subduction earthquakes.

\subsection{Dissertation Outline}

The present document is organized into five main chapters. Chapter 1 presents the research motivation, objectives and contributions of this study, as well as a literature review of the effects of subduction zone earthquakes on structural performance. Additionally, major subduction zone faults, retrofit measures and lessons from past megathrust earthquakes are briefly presented. Chapter 2 presents the analyses of a proposed inertial mass system designed for the experimental program part of this work. Moreover, the proposed system is numerically compared to two well-known existing inertial mass systems for shake table experiments. Chapter 3 presents the experimental program carried out for this study. It includes specimen's details and test setup, instrumentation and input ground accelerations. Also, this chapter presents the observed behavior and analysis of the experimental results for the six substandard circular RC bridge columns subjected to subduction and crustal demands through shake table test. Chapter 4 presents the numerical characterization and simulation of cantilever RC bridge columns based on the experimental results described in Chapter 3. The numerical simulations of the bridge columns are performed using OpenSees. Chapter $\mathbf{5}$ presents the fragility curves of a representative single-column RC bridge column. Based on the

results, preliminary performance limit states based on material strain are also presented in Chapter 5. Finally, conclusions and recommendations are presented in Chapter 6. 


\subsection{Retrofit Strategies for RC Bridge Columns}

Reinforced concrete bridges columns constructed before the 1970s were designed with no design consideration to seismic resistance and therefore, being deficient in flexural ductility, shear strength, and flexural strength. These failures are characterized by extensive and sometimes catastrophic damage. In particular, RC bridge columns included insufficient steel reinforcement to provide satisfactory performance in a major seismic event. Commonly, transverse steel consisted of No. 3 or No. 4 hoops spaced at 12 inches on center without considering cross-sectional column dimensions. Also, stirrups were anchored by $90^{\circ}$ hooks with short extensions, and intermediate ties were seldom used. Furthermore, reinforcing steel extending from the foundations was not sufficiently lap spliced to main column reinforcement or longitudinal reinforcement was premature terminated (Chai et al., 1991).

The deficiencies in detailing of pre-1970 bridge columns mentioned above make bridges highly vulnerable to a major seismic event, as it was observed the 1989 Loma Prieta and the 1994 Northridge earthquakes as shown in Figure 1.7 and Figure 1.7, respectively. Further, the recent occurrence of highly damaging subduction-zone earthquakes in Chile and Japan has demonstrated how vulnerable RC bridge columns are under major seismic events, as will be seen in the next section.

Even though the standards for earthquake design have been significantly improved, bridge columns constructed before 1970 are still part of important transportation lines, which may be prone to failure given their structural deficiencies. Under this scenario, retrofit programs have been undertaken over the past years in active 
seismic regions, aiming to minimize the likelihood of structural failure while meeting specific performance requirement. Thus, various retrofit and strengthening techniques have been developed, tested and implemented in RC bridge columns. Column retrofit techniques include steel jacketing, active confinement by wire prestressing, use of composite materials jackets involving fiberglass, carbon fiber, or other fibers in an epoxy matrix, and jacketing with reinforced concrete. These approaches are briefly discussed in the following.

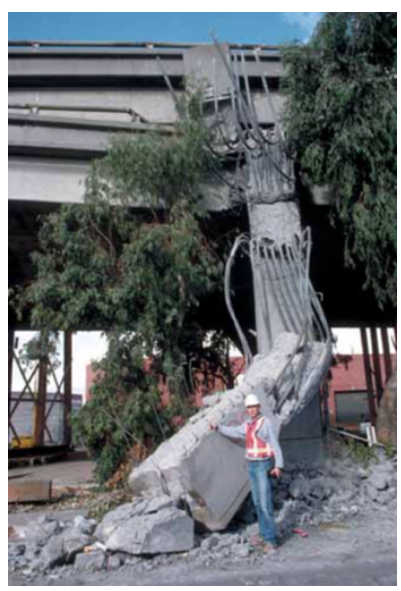

Figure 1.6 Support-column failure in Cypress viaduct during the 1989 Loma Prieta earthquake (USGS \& Wilshire, 1989)

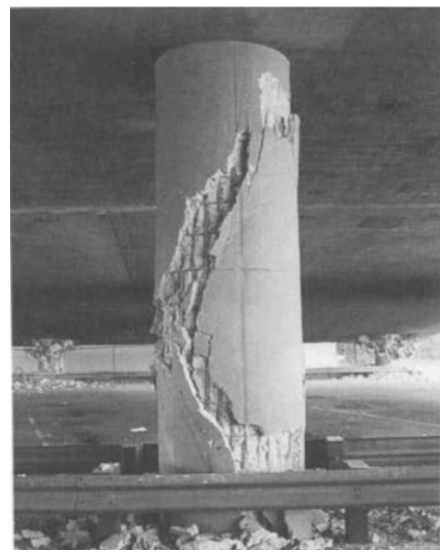

a)

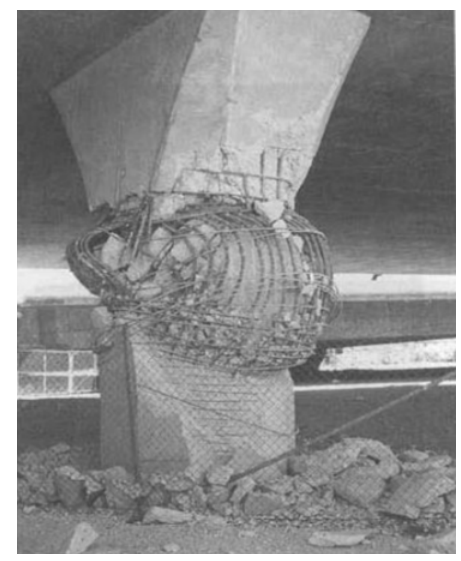

b)

Figure 1.7 Damage in RC columns, 1994 Northridge earthquake (Priestley et al., 1994). a) Freeway at Fairfax/Washington undercrossing, b) I-118 Mission/Gothic undercrossing 


\subsubsection{Concrete Jackets}

Concrete jacketing is one of the methods of choice for rehabilitation of deficient columns, although its use has been used primarily in existing columns in buildings. This method consists of encasing the existing reinforced concrete column with a jacket of concrete reinforced with longitudinal steel and drilled and grouted dowels or welded wire fabric. In general, this method is cheaper than other retrofit techniques, and it is also a suitable method for retrofitting columns in water. Figure 1.8 illustrates the concrete jacketing technique.

As for the effectiveness of this method, experimental investigations and field applications have shown that concrete jackets improve the strength and stiffness of asbuilt columns. However, an increase in column flexural strength leads to an increase in shear capacity, and therefore the forces transferred to cap beams and footing also increase (FHWA, 2006). As a consequence, undesirable effects can be generated on those components thus affecting the overall performance of the bridge. Confinement of circular columns is also enhanced using concrete jacket by using close-space hoops or spiral of small pitch. However, unless the concrete jacket is made of elliptical or circular shape, it is difficult to achieve effective confinement by rectangular concrete jacket.

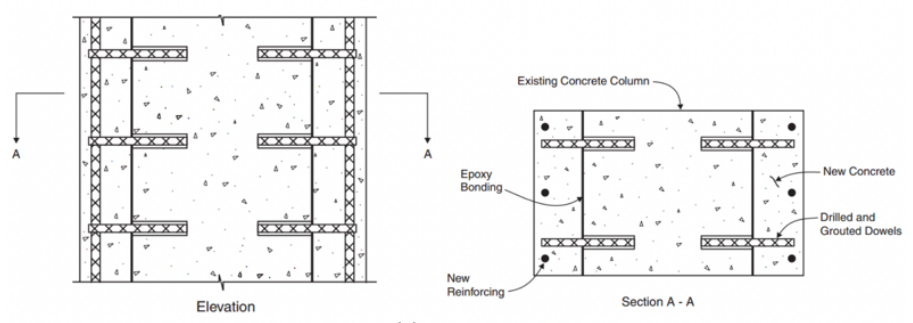

a)

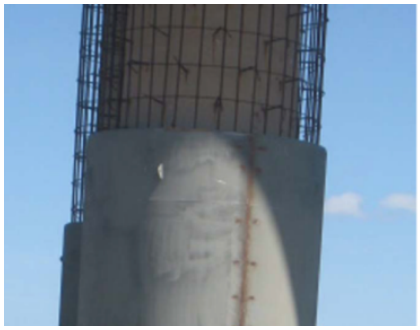

b)

Figure 1.8 Concrete jacketing. a) Concrete overlay (FHWA, 2006), b) Partial height concrete encasement of a column in Illinois (Poplar Street Complex) 


\subsubsection{Steel Jacketing}

Steel jacket has been used extensively throughout the United States, especially in California (Caltrans, 1996), and other active seismic regions to retrofit seismicallydeficient reinforced concrete bridge columns. Steel jacketing has been used as a retrofit measure to enhance the flexural ductility, shear strength, or performance of lap splices in reinforced concrete bridge columns. Its effectiveness in strengthening seismically weak columns has been supported by extensive research studies done in the past (Chai et al., 1991; Priestley et al., 1994). The procedure for circular columns consists of wrapping two half shells of steel plate around the existing column, leaving two vertical seams that are site-welded. The radius of the jacket is slightly oversized, usually with a radius of 0.5 to 1.0 in. larger than the column radius, and leaving a gap between the existing column and the inside of the steel shell. The void is filled with high-strength grout to provide composite behavior. The steel jackets are typically A36 steel casings and a space of about 2 inches is provided at the ends of the column to prevent the jacket from bearing on adjacent members. This serves to avoid undesirable flexural strength enhancement in which larger shears and moments may be transferred to the footings and cap beams under seismic loading (Priestley et al., 1996). While the effect is not intended, experimental testing by Chai et al. has revealed that the steel jacket increases column stiffness by approximately 10 to $15 \%$ for partial height (Chai, et al., 1991) and 20 to $40 \%$ for full

height jackets (Priestley, et al., 1996). This could undesirably impact the impact force and performance of bridge components, and is thus a critical consideration for analytical assessment of this retrofit. Figure 1.9 shows an application of this retrofit method. 


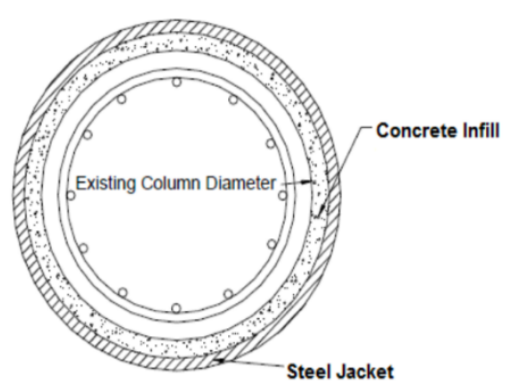

a)

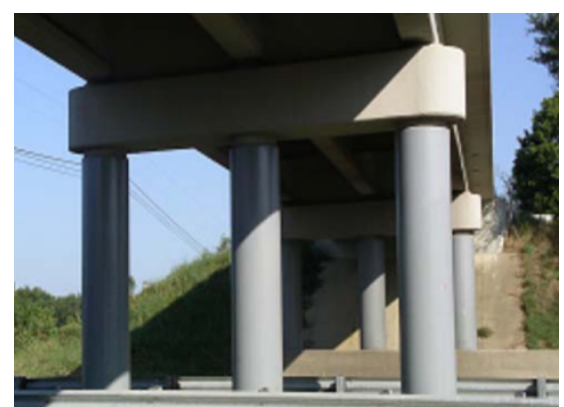

b)

Figure 1.9 Stell jacketing. a) method for circular column, b) Full column steel jacketing used in Tennessee (SR 196 over I-40)

\subsubsection{FRP Composite Jackets}

Another seismic retrofit technique used in RC bridge columns are based on composite materials bounded in an epoxy matrix and being the preferred one the Fiber Reinforced Polymers (FRPs). Among the different FRP composite materials, the most common include carbon fiber reinforced polymer (CFRP), glass fiber reinforced polymer (GFRP) and aramid fiber reinforced polymer (AFRP). An FRP typically consists of high tensile continuous fibers oriented in a desired direction in a specialty resin matrix. These continuous fibers are bonded to the external surface of the member to be strengthened in the direction of tensile force or as confining reinforcement normal to its axis. Fiber composite wraps may be continuous or applied in strips and can be effective on square as well as circular columns (Priestley et al., 1996). Figure 1.10 shows an application of FRP wrapping in a bridge. In contrast to typical retrofit techniques as steel and concrete jacketing, FRP wrapping provides many advantages, including very low weight-tostrength ratios, high elastic moduli, resistance to corrosion, and ease of application. Furthermore, studies have shown that FRP can improve column ductility without considerable stiffness amplification while maintaining the dynamic properties of the 
bridge (Haroun \& Elsanadedy, 2005). In is worth to mention that the Washington State Department of Transportation recommends using CFRP to retrofit bridges because it is less affected by moisture than GFRP (WSDOT, 2006).

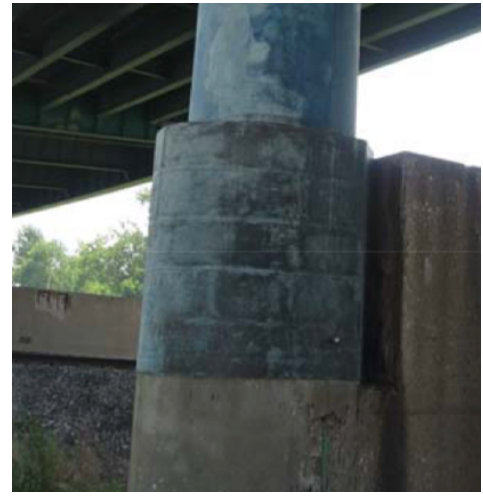

a)

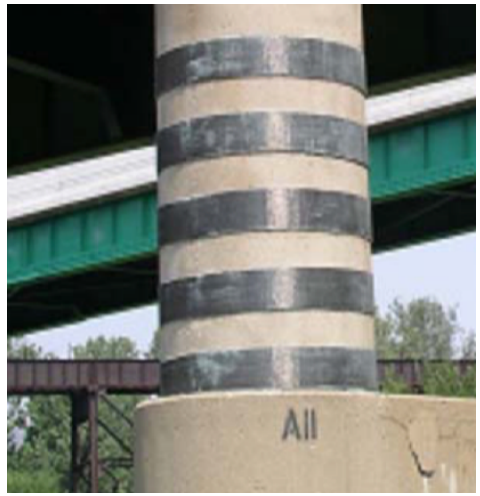

b)

Figure 1.10 Composite jacketing. a) continuous fiber composite wrap in Illinois, b) fiber composite wraps applied in strips in Illinois (Poplar Street Complex)

\subsubsection{External Prestressing Steel}

In this technique, prestressing tendons or bars are located according to predetermined profile on the external surface of the member to be strengthened according to design. Anchor heads are positioned at the ends of these tendons/ bars to post-tension the member using hydraulic jacks. This procedure has been reported to successfully increase the flexural ductility of circular columns with lap splices at the critical section, but its effect on shear has not yet been quantified (FHWA, 2006). Although, this method is quite effective by providing external confining forces into the column, it requires sufficient strength in the existing concrete to transfer the stress, and exposed tendons and anchorages need to be protected against corrosion. Figure 1.11 shows the application of this technique in a bridge. Regarding its effectiveness, research studies have concluded 
that this technique is effective for long columns were shear is not a significant failure mode (Coffman et al., 1991).

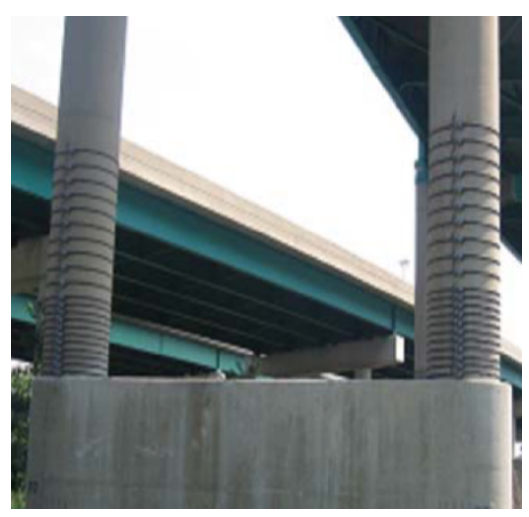

Figure 1.11 External prestressing steel retrofit of columns in a bridge in Illinois

\subsubsection{Smart Materials}

Smart materials have emerged as another option for retrofitting $\mathrm{RC}$ columns. The smart materials have unusual thermo-mechanical properties that have been explored for purpose of earthquake protection and retrofitting measures in structures. These materials, also called intelligent materials, have self-repairable and self-diagnosis characteristics. An example of these materials are shape memory alloys (SMAs), which have found application in bridge structures as an active confinement retrofit technique for $\mathrm{RC}$ bridge columns given the significant amount of energy that can dissipate due to shape memory effect (Andrawes et al., 2010).

\subsection{Lessons from Recent Subduction Zone Earthquakes}

Recent devastating, long-duration megathrust earthquakes occurred in the world emphasize the potential of having a severe longer duration, larger magnitude ground shaking than past events at some sites, for example, in the Pacific Northwest which lies 
near the Cascadia Subduction Zone. How structures, especially bridges, will perform under this expected seismic event is uncertain, given that many of the existing RC bridges were designed under old seismic provisions and there is a lack of acceleration records from past events to conduct research. However, recent strong seismic events have provided an extensive database of long-duration subduction-zone records to conduct research on this topic. In this section, lessons learned from reconnaissance trips to Chile and Japan after the mega events have provided more insight into the performance of bridges under subduction zone demands.

\subsubsection{Damage in Bridges due to the 2010 Chile earthquake.}

On February 27th, 2010, Chile was shaken by one of the largest magnitude earthquakes $(\mathrm{Mw}=8.8)$ ever recorded (Boroscheck et al., 2012). The structural performance of bridges was better than that expected despite the large magnitude and rupture extension of the earthquake. It was registered that 211 bridges were damaged in a highway network of more than 7,250 bridges (Gobierno de Chile, 2010). The damages vary from slightly to complete collapse. Detailed damage of Chilean Bridges after the earthquake can be found elsewhere (Kawashima et al., 2010; the Federal Highway Administration (2011); and Bubkle et al., 2012). One of the most common observed damage was due to unseating of the superstructure spans in skewed bridges. It is known that the entire deck in skewed bridges can rotate about a vertical axis during seismic excitations, which would result in unseated superstructures at the acute corner as is shown in Figure 1.12 and Figure 1.13. As a result, the demands are not transferred to the bridge substructure, and therefore very low or even no damage occur in the columns. 
Reconnaissance teams mainly attributed this mode of failure to the absence or weakness of constraints to prevent rotations, such as side stoppers and diaphragms, and to insufficient support length. This damage feature would had been prevented under current Caltrans seismic criteria, which requires balanced structures with large seats, good continuity of reinforcement, and well-confined ductile members (Caltrans, 2013).

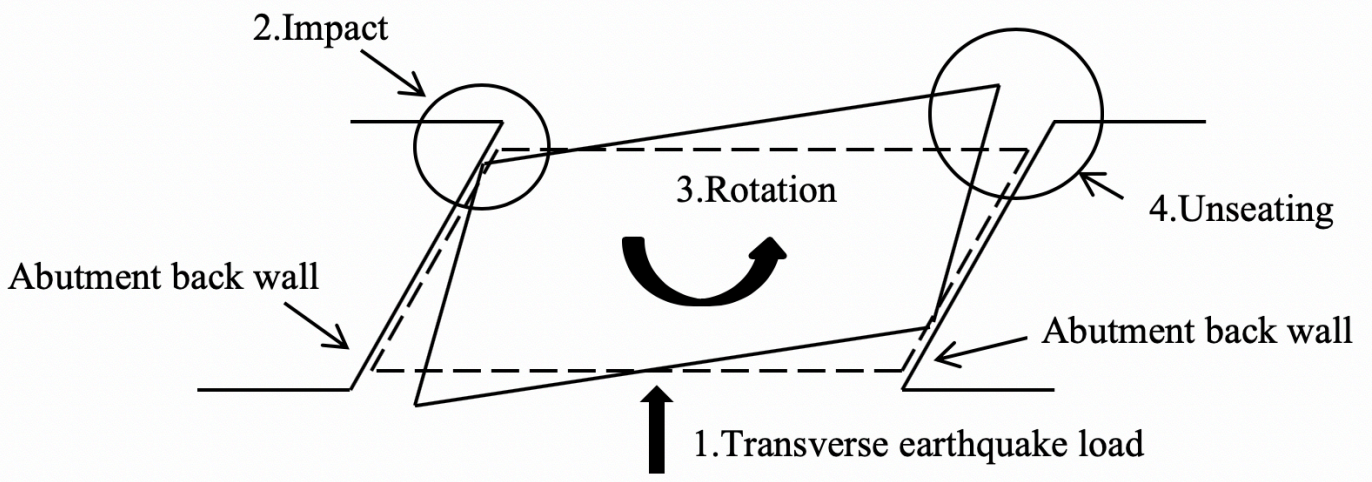

Figure 1.12 Deck rotation and unseating in skewed bridges (plan view single span)

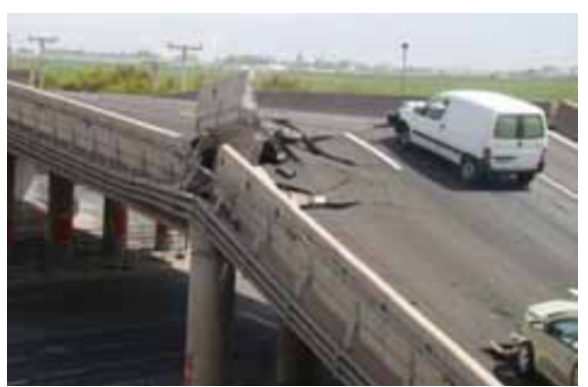

a)

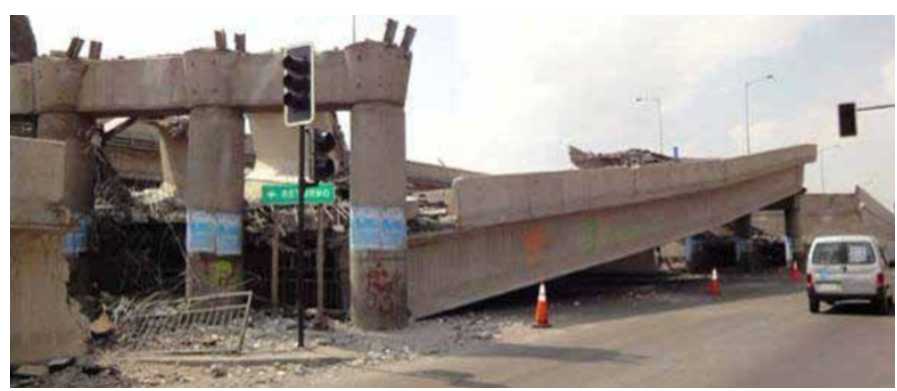

b)

Figure 1.13 Collapsed bridge due to in-plane rotation and unseating of the deck. a) Miraflores Bridge, b) Lo Echevers Bridge

Damage to bridge columns and cap beam was observed due to shear and flexure failures in bridges which were unreinforced or constructed in early days, as shown in Figure 1.14. In the damage shown in Figure 1.14, the shear capacity of the concrete was probably overestimated in those days. Further, soil liquefaction induced ground 
movement that contributed the initiation of this failure. Except those bridges built in the early days, bridges built in recent years did not suffer extensive damage at their columns.

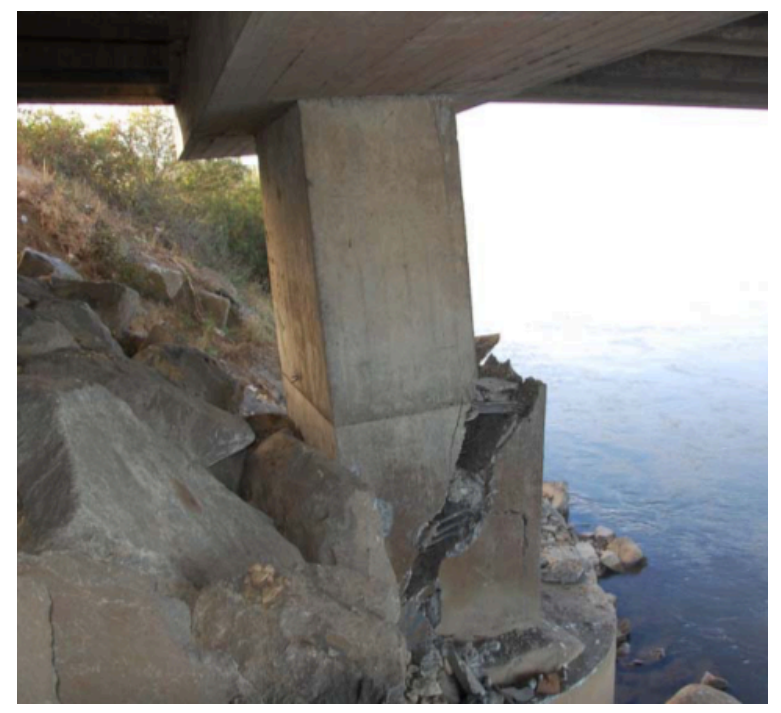

Figure 1.14 Damage in RC bridge column due to insufficient shear capacity

At the time of the earthquake, the Chilean seismic design was governed by the Manual of Highways requirements (Ministerio de Obras Públicas, 2002). It is worth to mention that the minimum seat width at the abutments and bents was that described in AASHTO (1996). Another important aspect is that the pre-earthquake Chilean code allowed the designer to eliminate the diaphragms in seismic zones of lower risk if an adequate bridge performance could be demonstrated (Schanack et al., 2012). For this reason, many of the collapsed bridges did not have end diaphragms. Post-earthquake, the Road Administration of the Chilean Ministry of Public Works (MOP), based on the observed bridge damage, incorporated new requirements for the minimum seat width, stopper structures, and skew angle limits (Unjoh, 2012). These requirements were based on the Japanese Specifications for Highway Bridges (Japan Road Association, 2002). An application of these modifications can be observed in the new construction of Miraflores 
Bridge and Lo Echevers Bridge, which were reconstructed by incorporating shear keys between the girders, diaphragms, and longer transverse seat width as illustrated in Figure 1.15 .

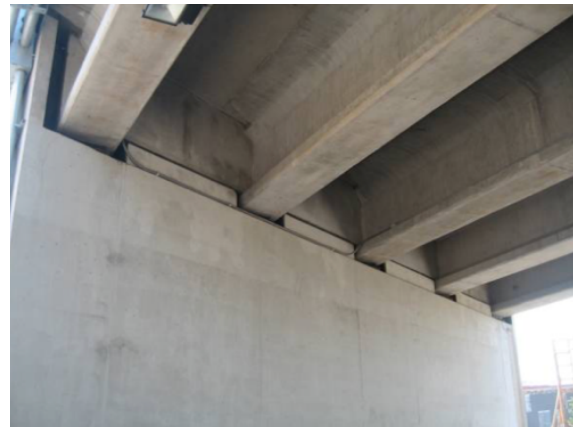

a)

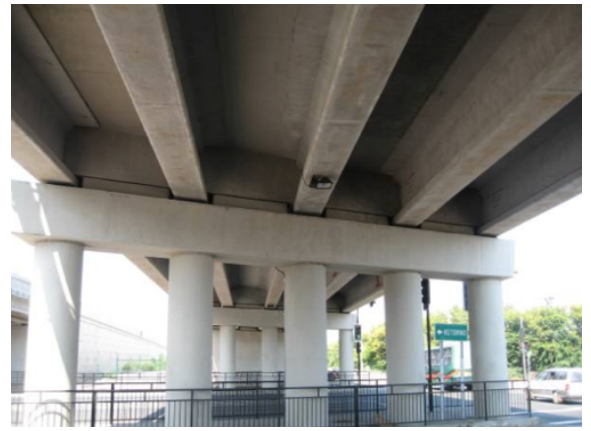

b)

Figure 1.15 Repair and retrofit for Miraflores bridge (Unjoh, 2012). a) Lateral stopper at abutment, b) lateral beam and lateral stopper at pier

\subsubsection{Damage in Bridges due to the 2011 Tohoku earthquake}

The 2011 Great East Japan (Tohoku) earthquake (Off Pacific Coast of Tohoku Region) with a magnitude of Mw 9.0 occurred on March 11, 2011, along the Japan Trough in the Pacific. It is one of the largest earthquakes ever recorded in the world, with a fault zone extended $450 \mathrm{~km}$ and $200 \mathrm{~km}$ in the north-south and west-east directions, respectively as shown in Figure 1.4. Extensive damage occurred in the vast region in the east part of Japan (JSCE, 2011). Over 1,500 highway bridges were damaged during the earthquake, where the causes of damage of these highway bridges can be classified as effect of strong ground shaking, including soil liquefaction, and effect of tsunami inundation. A complete list of damaged bridges and mode of failures can be found elsewhere (Kawashima et al., 2011; Kawashima \& Buckle, 2013; Hoshikuma \& Guangfeng, 2013). Most of the observed damaged due to ground motion was developed mainly in bridges not yet retrofitted or only partly so. These bridges were designed with 
pre-1990 design specifications and the damage modes registered had been observed in past earthquakes. One of the common observed and registered damage in RC columns without retrofit was the flexural-shear failure of RC columns as shown in Figure 1.16. This damage occurred due to an overestimated concrete shear capacity and an inadequate development length of longitudinal bars at section cut-off, which were the common practice prior to 1980 . Such a failure occurred extensively during the 1995 Kobe earthquake (Kawashima \& Unjoh, 1997), but seismic retrofit projects undertaken thereafter took care of these existing bridges designed in accordance with pre-1980 specifications with high priority in an effort to prevent the collapse of the bridge structure and unseating of the deck. Bridges which had been retrofitted suffered virtually no damage due to effect of ground motion, which revealed the effectiveness of the post-1990 specifications for seismic retrofit. Additionally, new bridges constructed in accordance with post-1990 codes suffered essentially no damage during this event, except for some damage in elastomeric bearing in a bridge (Kawashima \& Buckle, 2013).
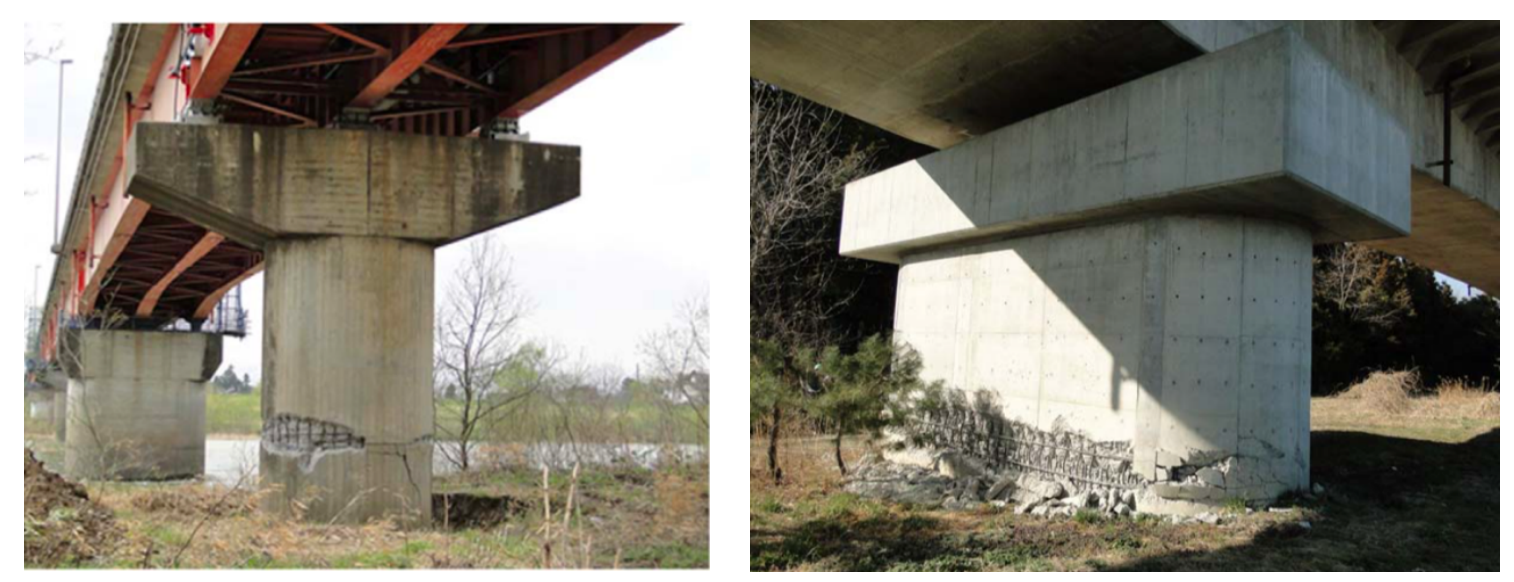

Figure 1.16 Damage of RC column at section of cut-off of longitudinal reinforcement 


\section{CHAPTER 2. Analysis of Inertial Mass Systems for Shake Table Experiments}

\subsection{Introduction}

This research was focused on studying the seismic performance of reinforced concrete bridge columns subjected to real-time earthquake motions. Such dynamic-type excitations can be reproduced using a shake table system. This system has the advantage of reproducing the recorded accelerations of a real earthquake with high reliability, and it also has the ability to reproduce inertial effects that cannot be correctly modeled on a quasi-static or pseudo-dynamic test. Despite the advantages of the shake table systems to accurately represent the earthquake response of structures, limitations of the size and weight of the tables, have made the dynamic testing of reduced-scale models or testing only the main structural components usually necessary. Under this scenario and to comply with modeling requirements, a large amount of mass should be added to the test specimen. Plus, inertial loading system proposed to date have not addressed all the safety, setup times and costs. Therefore, a new inertial mass system is proposed to minimize the deficiencies in current shaking table testing setups. The proposed system was developed as part of this study to test on shake table single cantilever-type columns under dynamic actions.

In this chapter, the proposed inertial mass system (IMS) for shake table tests of cantilever RC columns is presented. Additionally, a review and discussion of current inertia mass systems is included. System characteristics are discussed in detail, and the dynamic equations of motion of the proposed inertial mass system and shake table are 
developed. The proposed system has been used successfully in the dynamic testing of six cantilever-type reinforced concrete bridge column models. The experimental verification of the proposed system is saved for Chapter 3, where the experimental program part of this study is discussed in detail. Nonetheless, the new inertia system is numerically compared to two different and prominent mass setup systems used for shaking table tests of cantilever RC columns.

\subsection{Inertia Systems for Shake Table Tests}

Shake table testing is a valuable tool for the seismic behavior assessment of structural components, substructures or entire structural systems. However, high costs of installation and maintenance and laboratory capabilities limit the size of shaking tables (Caccese \& Harris, 1990). Then, simplifications become necessary such as testing reduced-scale models or the main components of a structural system. If reduced-scale models are used, additional mass is often required to set the natural period of the specimen corresponding to that of the prototype structure or in the case of tests aiming at assessing the performance near or at the collapse performance level. Additional mass attached directly on the top of specimens, external mass systems using linear sliding, rotational and pendulum systems, as well as systems using mass on the table have been used in several experimental programs. All of these configurations have diverse advantages and drawbacks, providing a wide range of solutions to different types of research with diverse objectives. Their benefits and limitations are discussed below. 


\subsubsection{Inertial Mass Supported by Specimen}

Several shake table tests on cantilever RC columns have been conducted by placing the inertial mass directly on top of the specimen and subjecting it to the intended ground motion. Among these experiments are tests performed on cantilever RC columns by MacRae et al., 1994; Mosalam et al., 2002; Hachem et al., 2003; Sakai et al., 2005; Schoettler et al., 2015, among others. In this configuration, it is possible to reproduce the axial force on the column model produced by the superstructure weight, as well as the Pdelta effect. Even though the use of this method of placing the inertial mass has been widely used, there are important shortcomings associated with it. One of the main drawbacks of this configuration is that leads to delays and more costs due to mass removal and reinstallation when more than one specimen has to be tested. Moreover, an auxiliary-supporting structure must be considered for safety during and after testing in the occurrence of large displacements, or a likely collapse of the specimen. Figure 2.1 and Figure 2.2 show two setups including the inertial mass directly on top of the test specimen.
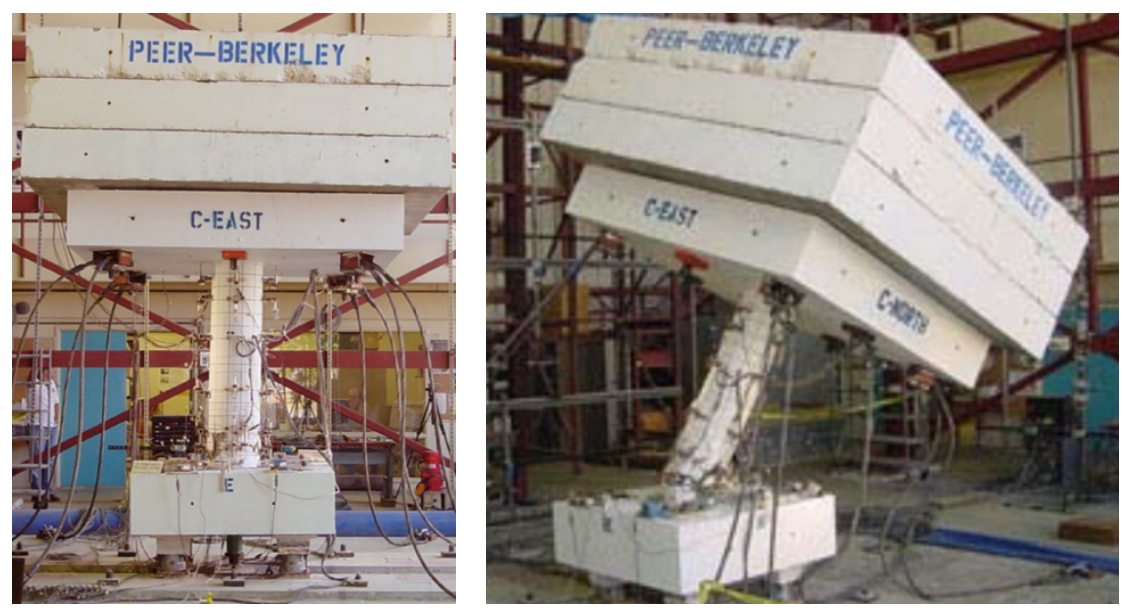

Figure 2.1 UC-Berkeley inertial loading system (Sakai et al., 2005) 

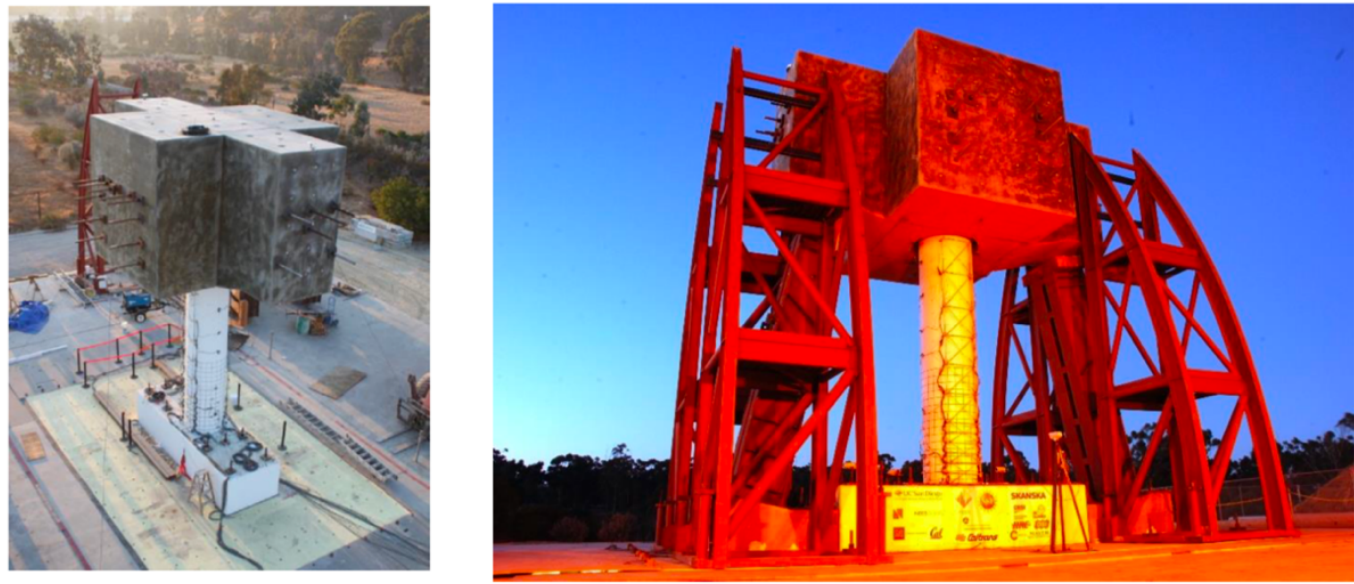

Figure 2.2 UC-San Diego inertial loading system (Schoettler et al., 2015)

Another disadvantage of this configuration is the size of the inertial mass system, which can be somewhat large as compared to the specimen, as seen in the figures. The large size of the blocks could result in additional higher mode effects associated with rotational deformation demands at the top of the specimen. It is worth mentioning that these demands are not encountered in field conditions since the inertial mass tributary to a given column is constrained in the rotational degree of freedom.

\subsubsection{Inertial Mass Supported by External System}

Another configuration for including the additional mass in shake table testing is to locate it outside the table. In this configuration method, the additional mass is placed on a fixed supporting structure beside the shaking platform, and it is linked to the test specimen through a pinned-end connection that allows free rotations and therefore transmitting axial force only. Relevant advantages are associated with this configuration (Carrillo et al., 2012). Taking the additional mass off from the platform reduces the risk of damaging the test equipment and laboratory personnel if lateral instability of the 
specimen is reached during testing. Another advantage of this method is the reduction in the time necessary to assemble and disassemble a test setup. This is because of the simple connection between the mass-rig and the specimen only needs to be disconnected from the model. Therefore, if the experimental program includes a large number of tests, research time decreases considerably. Additionally, another benefit from this configuration is that it allows for using the full performance and capacity of the shake table because shaking table performance depends on the weight acting on its test platform.

Three types of mass-rig systems located outside the table have been used: a linear sliding system, a rotational system, and a pendulum system. Among these systems, the rotational mass-rig has been used in experiments involving $\mathrm{RC}$ columns as the specimen, as the one proposed by Laplace et al. (1999). This mass rig system is reproduced in Figure 2.3 and consists of a horizontally constraint-free mechanism to provide the inertial dynamic loading during the testing procedure. The axial load applied to the column is achieved through a steel spreader beam attached to the top of the specimen and two center-hole rams. Restraining cables are provided to restrict the translation of the inertial mass. In the case of column failure, the mass-rig would translate until the maximum displacement preset by the restraining cables is reached. Thus, safety concerns are eliminated if the specimen fails. Further, the mass-rig restrain out-of-plane movement of the specimen, and therefore additional elements for this purpose are not necessary. As indicated before, another advantage of this mass-rig system is that also allows specimen installation and removal with easiness. 


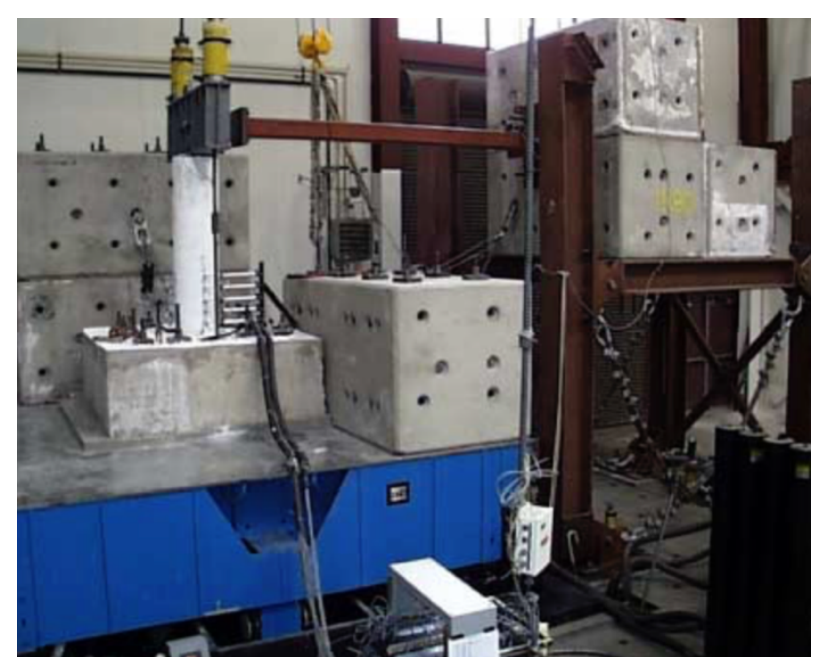

a)

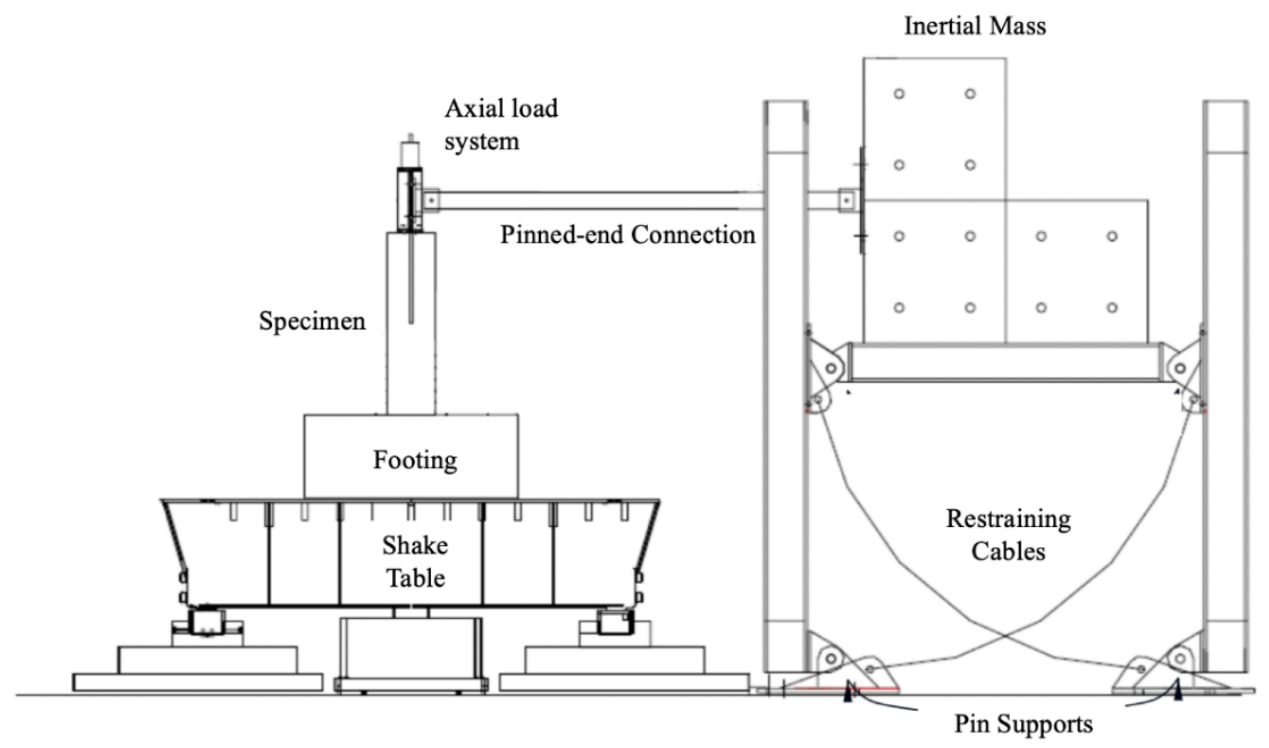

b)

Figure 2.3 Unidirectional Mass-rig at UNR (Laplace et al., 1999). a) Actual test setup, b) Adapted scheme

Although this system eliminates various concerns related to shake table testing of cantilever columns, some issues related to the correct representation of P-delta effects are included. Laplace et al. (1999) concluded from their experimental results that the experimental shear forces were smaller than those predicted from the analysis including P-delta effects. 
Conclusions from these two types of tests indicate that (1) placing a large volume mass on top of a column leads to higher mode effects that are not typically observed in an actual bridge configuration, and (2) placing the inertia mass on an external mass-rig system does not properly represent the P-delta effects that cantilever columns experience during ground motions. In an effort to overcome these two concerns, a new inertia mass system (IMS) is proposed. In this system, the additional inertia mass is supported by the shake table employing a stiff-pinned column attached to the platform. System features are discussed in detail, and the dynamic equations of motion of the internal system and the shaking table are developed. It is worth mentioning that the proposed system has been used successfully in the dynamic testing of six reinforced concrete bridge column models, which will be presented in Chapter 3.

\subsection{Proposed Inertia Mass System}

\subsubsection{System Description}

A new inertial mass system, IMS, located on the shaking table platform, was developed as part of this study, to enable the shake table testing of single cantilever-type reinforced concrete columns subjected to unidirectional earthquake excitations. A schematic of the proposed system is depicted in Figure 2.4. The IMS consists of a steel column pin-connected to a supporting W-beam which, in turn, is connected to the shaking platform utilizing four high-strength post-tension rods. Required inertial mass was incorporated into the system by using a predefined quantity of concrete blocks, each one with an approximate weight of $9560 \mathrm{lb}$. In total four concrete blocks were used for this study, which were attached to the steel column via high strength post-tension rods at 
a height dictated by the test specimen's height. The IMS is connected to the specimen's head through a pinned-end rigid link. The link was designed to transfer the inertial forces created on the inertial mass system to the specimen allowing only in-plane rotations. For that, 50 kips swivels were added at the ends of a rectangular structural section (HSS4x3x1/4"). A load cell was mounted in the link to measure the lateral force transferred to the specimen. Additionally, to ensure that the concrete blocks remain joined, and the inertial forces are being transmitted through the rigid link while shaking, a W-beam was added at the back of the concrete blocks using various post-tension rods. It is worth mentioning that, one end of the pinned rigid link was post-tensioned to the $\mathrm{W}$ beam attached to the IMS.

A safety system external to the shake table system was designed to catch the IMS in the event of large displacements or specimen collapse. The safety frame consisted of six columns and bolted beams; each of the steel columns was fixed to the laboratory strong floor using high strength bolts. The two closer bays of the safety frame were braced together using a series of angle braces in a cross shape in the north-south direction (Figure 2.4 a)), whereas chevron bracing was used in the east-west direction (Figure 2.4 b)). It is important to mention that the motion on the platform is stopped when the concrete masses impact against the longitudinal beams of the safety frame. A maximum drift of 25 in is allowed by the safety system in the direction of motion. To preclude outof-plane motion of the IMS, a caster was attached to the web of each longitudinal beam, as shown in Figure 2.4.b. 


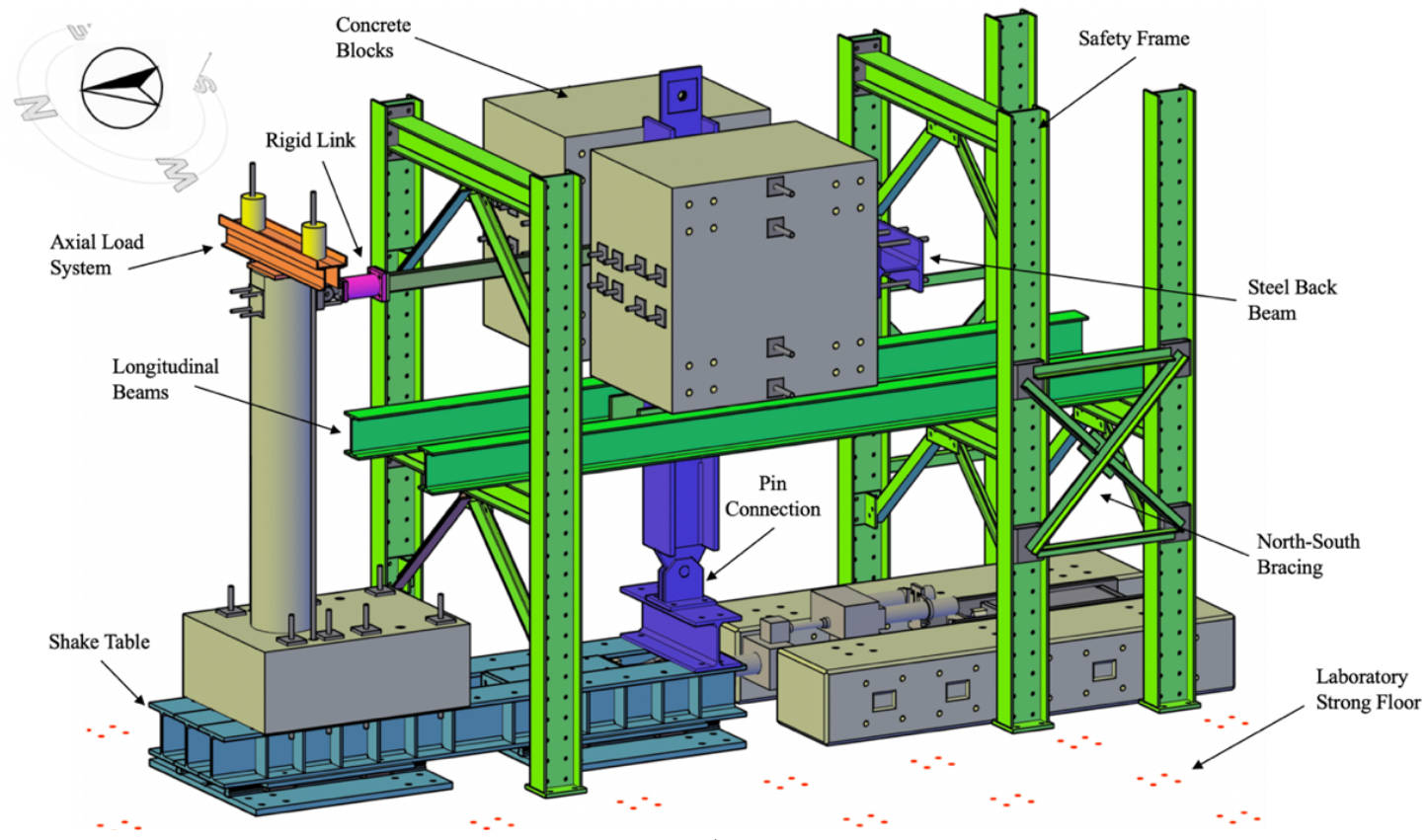

a)

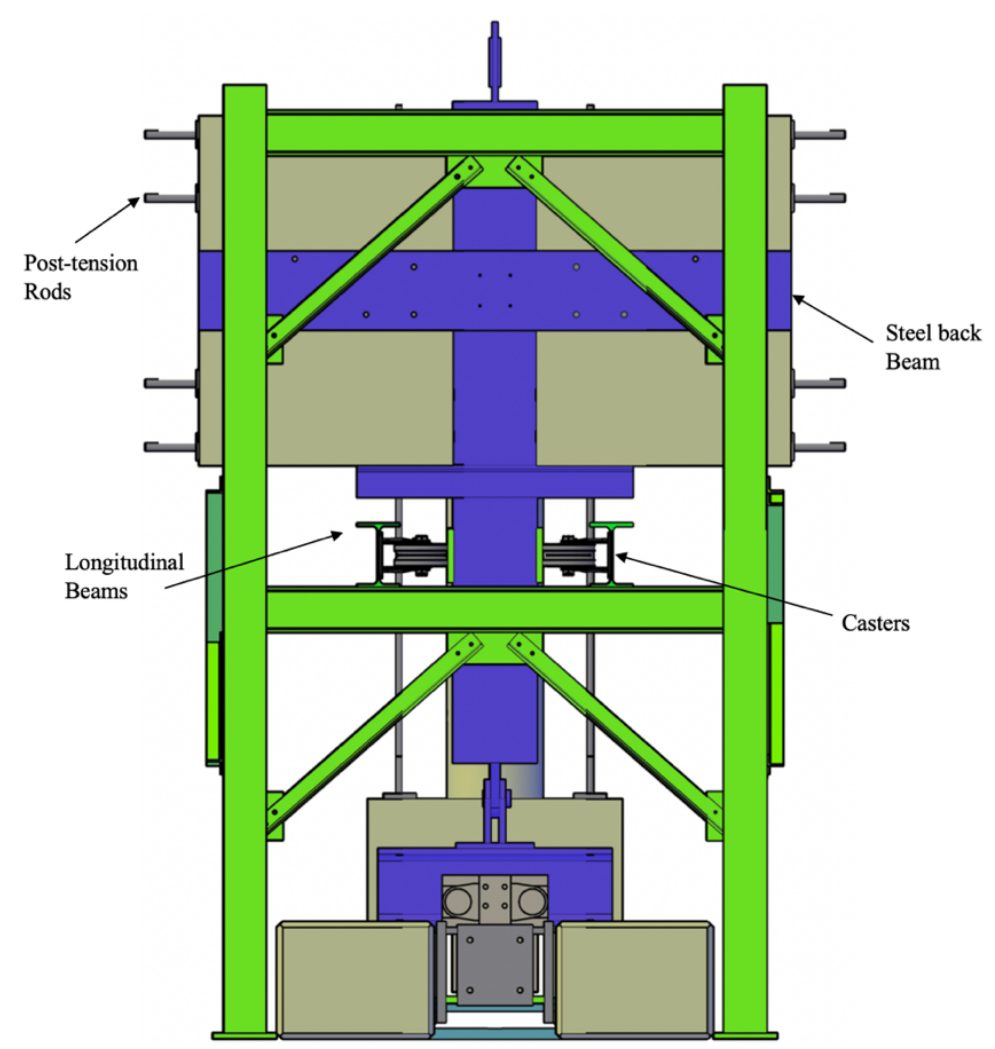

b)

Figure 2.4 Schematic of the IMS part of test setup. a) 3D-view, b) South view 
Since the IMS was composed only by a steel column and masses, the setting process of an RC specimen can be completed in a short time. As is illustrated in Figure 2.5, the process of assembling the IMS includes the following steps: a) attaching the supporting W-beam with the clevis bolted onto it to the shaking platform (Figure 2.5.a); b) erecting the steel column and connecting it to the clevis (Figure 2.5.b); c) attaching the concrete blocks to the steel column and placing the steel back beam at the required height (Figure 2.5.c); d) setting the RC specimen by securing its footing to the shake table with high strength post-tensioning rods; e) connecting the links between the steel column and the head of the RC specimen; f) attaching the axial load system on top of the RC column.

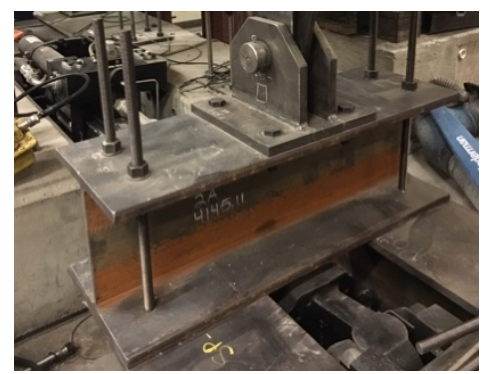

a)

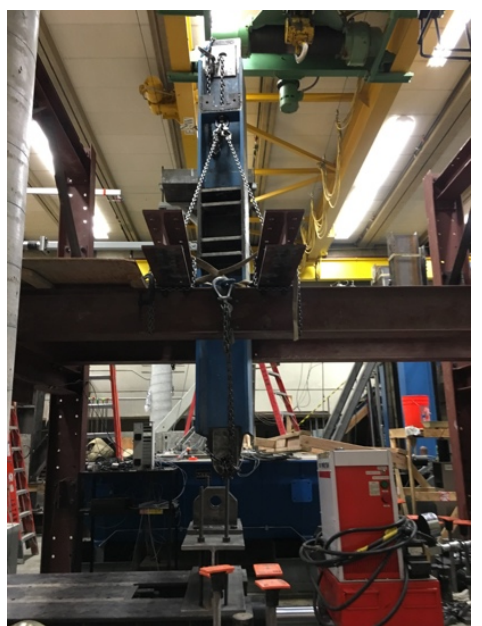

b)

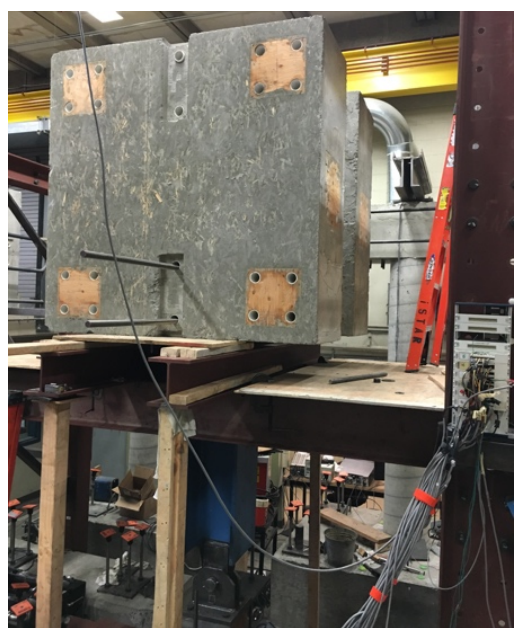

c)

Figure 2.5 Inertial Mass System (Assembling Process)

\subsubsection{Advantages and Disadvantages}

There are important advantages of the proposed inertia mass system: (1) Safety: during column failure or if the specimen is tested to collapse performance level, large displacement may occur. Under this scenario, the safety catch frame restrains the displacement of the mass (Figure 2.6). Then the safety of laboratory personnel, 
equipment, and specimen instrumentation is also improved. Conversely, if the inertial masses were placed directly on top of the specimen, during column failure and fall of the concrete blocks, permanent damage to column instrumentation and even probably shake table system components could happen; (2) Preparation time: since the connection between the masses and specimen is simple, time for assembling and disassembling the test setups decreases. Before testing, the IMS rests on the safety catch frame using hydraulic rams as supports. Once the specimen is placed on the shake table, the masses are lifted by applying pressure to the rams and then connecting the rigid link to the specimen's head. Therefore, when an experimental program considers various tests, research time decreases considerably; (3) Small out-of-plane displacement: using the pinned-end rigid link with only free in-plane rotations allowed (transverse rotation restrained), the out-of-plane displacements are diminished or almost eliminated. However, to assure pure in-plane behavior, one caster was attached to each longitudinal beam of the safety catch frame to restrain the out-of-plane displacements of the IMS. The steel column is provided of two steel plates (one on each side) where the casters slide if out-of-plane displacements would be present. It is worth noting that the gap between the plates and the caster was only $3 / 16$ inches on each side. 


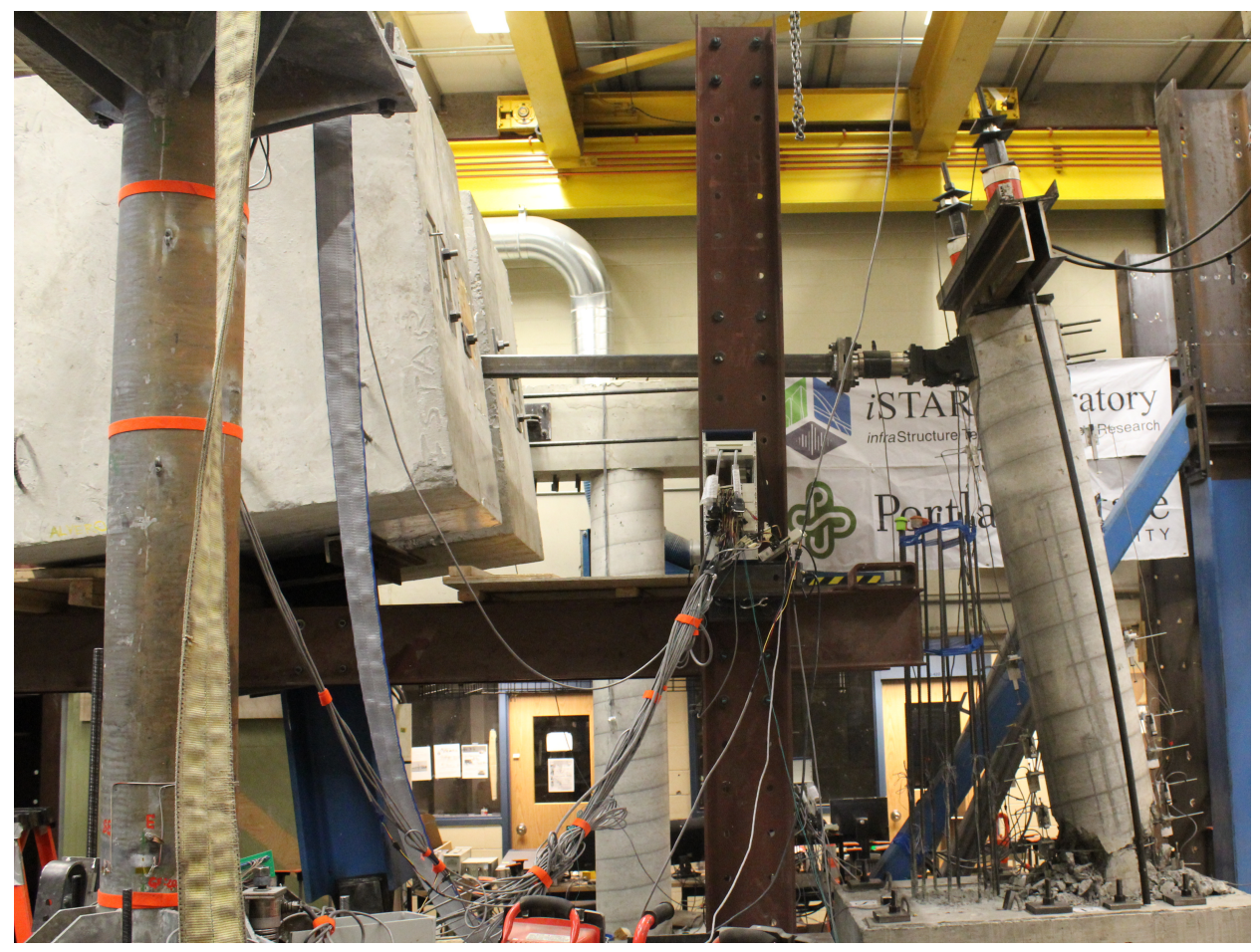

Figure 2.6 IMS reaching maximum displacement limit under specimen collapse

The main disadvantage of the proposed inertia system is that the performance and full capacity of the shake table may not be used if the additional inertial mass was rather large compared to the one considered in the proposed system. This situation is because shake table performance depends on the weight acting on its platform (Bairrao \& Vaz, 2000). Additionally, by placing the inertial mass on the test platform, significant overturning moments are introduced to the system, and therefore imposing a significant challenge for proper control in the closed-loop system. Another drawback of the proposed system is that axial forced needed to simulate superstructure weight in case of bridge columns are not applied. Therefore, an additional system is needed to overcome this problem. For the proposed system, axial loads on the specimen were applied through two high-strength rods and hydraulic rams. 


\subsubsection{Equation of Motion}

As it was discussed in the previous section, the IMS greatly simplified the test setup and preparation of specimens. Nevertheless, it has an impact on the loading and stiffness of the specimen through the P- $\Delta$ effect at large displacements. The relevance and magnitude of this effect can be analyzed through the equation of motion for the whole system depicted in Figure 2.7. The equation of motion for a nonlinear single degree of freedom oscillator with constant viscous damping (Chopra, 2001), in response to horizontal earthquake motion, can be written as:

$$
m \ddot{x}(t)+c \dot{x}(t)+k(t) x(t)=-m \ddot{x}_{g}(t)
$$

Where $m$ is the mass of the oscillator, $c$ is the damping coefficient, $k(t)$ is the stiffness, $x, \dot{x}$, and $\ddot{x}$ are the displacement, velocity and relative acceleration of the oscillator, respectively, and $\ddot{x}_{g}$ is the ground acceleration.

For the system shown in Figure 2.7, Eq. (1.1) can be rewritten as:

$$
m \ddot{x}(t)+c \dot{x}(t)+\left(k(t)-\frac{W^{\prime \prime}}{H}\right) x(t)=-m \ddot{x}_{g}(t)
$$

For Eq. (1.2), $m$ is the lateral effective mass of the system and it is equal to:

$$
m=m_{1}+m_{2}+m_{3}+\frac{m_{4}}{2}
$$

Where $m_{1}$ is the IMS mass, $m_{2}$ is the mass of the pinned-end rigid link system, $m_{3}$ is the mass of the axial load system on column, $m_{4}$ is the specimen mass. $H$ is the height of the specimen and $W^{\prime \prime}$ is the IMS effective weight which, in turn, is calculated as: 


$$
W^{\prime \prime}=\left(m_{1}+\frac{m_{2}}{2}\right) g
$$

Where $g$ is the gravity acceleration $\left(386.4 \mathrm{in} / \mathrm{s}^{2}\right)$. The total displacement of the system, $x_{a b s}$ is defined as:

$$
x_{a b s}(t)=x(t)+x_{g}(t)
$$

Where $x(t)$ is the relative column displacement and $x_{g}(t)$ is the shake table displacement. The quantities of the above variables are listed in Table 2.1.
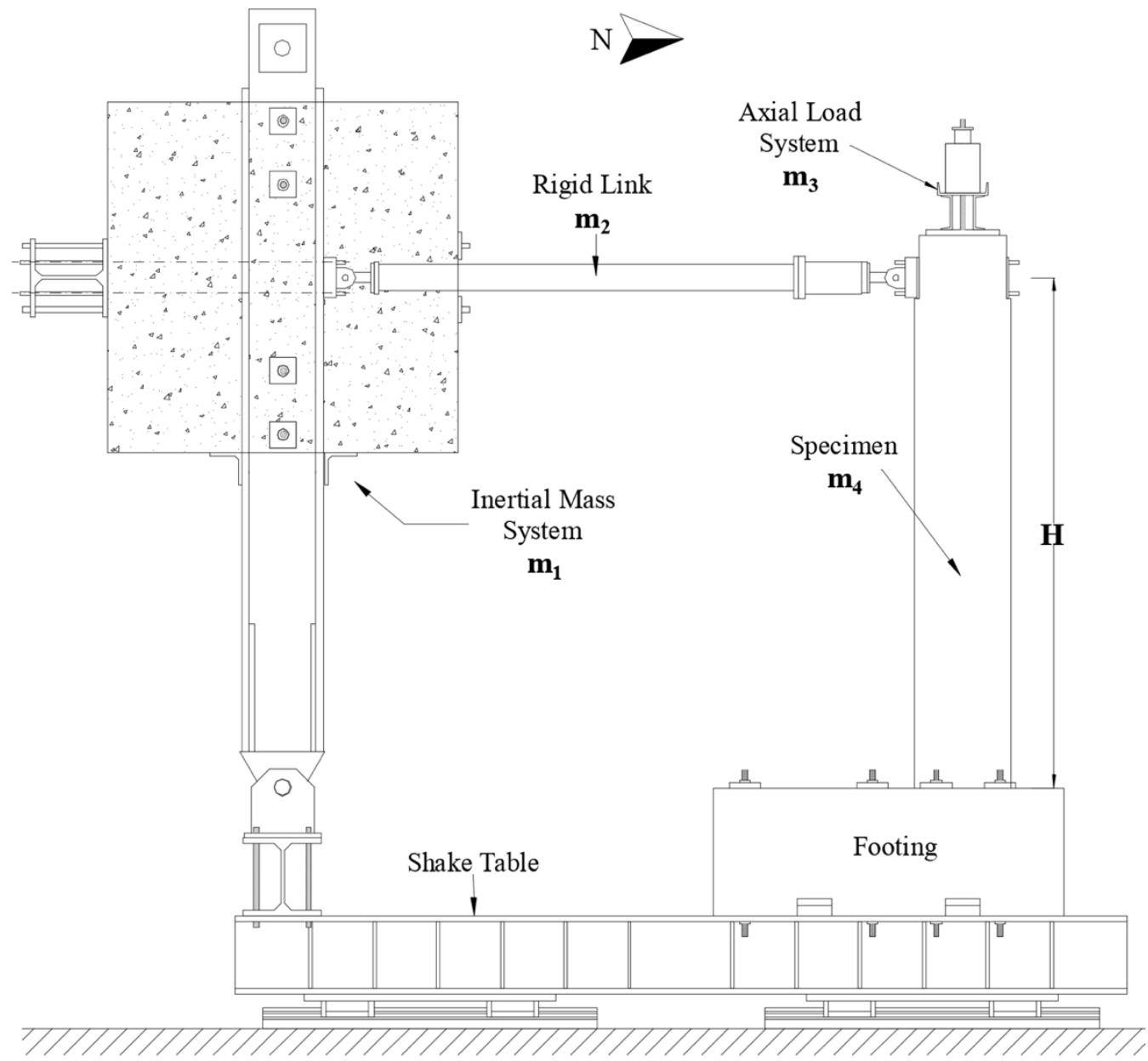

Figure 2.7 IMS mass locations 
Table 2.1 Masses and dynamic weights of the IMS

\begin{tabular}{l|c|c}
\hline \multicolumn{1}{c|}{ Component } & $\begin{array}{c}\text { Mass } \\
\left(\mathrm{lb}-\mathrm{s}^{2} / \mathrm{ft}\right)\end{array}$ & $\begin{array}{c}\text { Weight } \\
(\mathrm{lb})\end{array}$ \\
\hline $\begin{array}{c}m_{1}=4 \text { concrete blocks, W-beam, } \\
\text { portion of steel column mass }\end{array}$ & 1258 & 40,510 \\
\hline$m_{2}=$ rigid link system & 9.3 & 300 \\
\hline$m_{3}=$ axial load system & 16.1 & 520 \\
\hline $\begin{array}{l}m_{4}=\text { distributed mass of specimen } \\
\text { contributing to effective inertia }\end{array}$ & 72.7 & 2340 \\
\hline$\psi_{1}$ & \multicolumn{2}{|c}{0.22} \\
\hline
\end{tabular}

\subsubsection{Calculation of Lateral Force}

The lateral force acting on the test specimen $\left(F_{c o l}\right)$ is the sum of the spring and damping forces:

$$
F_{c o l}(t)=k(t) x(t)+c \dot{x}(t)
$$

The force acting on the test specimen $\left(F_{c o l}\right)$ may be obtained by applying one of three procedures, namely, using a load cell, through the equation of motion, or using an accelerometer.

\subsubsection{Using Load Cell}

The pinned-end rigid link (Figure 2.4) was instrumented with a 50 kip load cell which was placed just before the swivel attached to the column's head and connected to data acquisition system. The load cell measures the lateral force acting on the specimen due to the inertia force from the inertial mass system and inertial mass system P- $\Delta$ force due to the overturning effect. However, it does not include the inertial mass of the pinned-end rigid link between the load cell and specimen, the axial load system, and the 
contribution of the specimen to the inertial force. Therefore, the lateral force acting on the column is defined as:

$$
F_{c o l}(t)=F_{L C}(t)+\ddot{x}_{a b s}(t)\left(\psi_{1} m_{2}+m_{4}\right)
$$

Where $F_{L C}$ is the force measured by the load cell, $\ddot{x}_{a b s}$ is the absolute acceleration measured in the specimen and $\psi_{1}$ is the percentage of mass of the link system between load cell and specimen. $F_{c o l}$ and $\ddot{x}_{a b s}$ are positive in the direction towards the specimen.

\subsubsection{Using the Equation of Motion}

The second option for calculating $F_{c o l}$ is through the equation of motion. Replacing Eq. (1.6) into Eq.(1.2) and rearranging terms:

$$
F_{c o l}(t)=-m\left[\ddot{x}_{g}(t)+\ddot{x}(t)\right]+\frac{W^{\prime \prime}}{m \cdot H}
$$

According to Eq.(1.5), Eq.(1.8) can be rewritten as:

$$
F_{c o l}(t)=-\left[m \cdot \ddot{x}_{a b s}(t)-\frac{W^{\prime \prime}}{H}\right]
$$

It is worth noting that the lateral force $\left(F_{c o l}\right)$ calculated either using Eq.(1.7) or Eq.(1.9) must be adjusted to account for the effects of the horizontal component of the applied axial load at large displacements, as described in next section.

\subsubsection{Calculation of P-Delta Force}

The $\mathrm{P}-\Delta$ effect was defined as the equivalent lateral force due to the overturning moment that is equal to the vertical force multiplied by lateral drift. There are two components contributing to the P- $\Delta$ in this study. First, the P- $\Delta$ created from the 
overturning moment of the inertial mass system. To calculate the equivalent lateral force due to the inertial mass system, the effect was defined as:

$$
P \Delta_{I M}(t)=\frac{W^{\prime \prime}}{H} \cdot x(t)
$$

Since Eq. (1.10) is included in the force measured by the load cell, the lateral column force remains the same in Eq.(1.7).

The second P- $\Delta$ effect was due to the axial load system. The effect depends on the axial load line-of-force, which in this study is pivoting near the base of the footing, as shown in Figure 2.8. To resulting P- $\Delta$ effect can be calculated by the following:

$$
M_{P \Delta}=P^{\prime} x(t)
$$

Where $P$ is the applied axial force, $P^{\prime}=P \cos (\alpha)$ is the vertical load, and $P \sin (\alpha)$ is the restoring force of the post-tensioning bar. As shown in Figure 2.8, it is clear that the effective lateral force to the column is modified by the restoring force of the posttensioning bar:

$$
F_{c o l, e f f}(t)=F_{c o l}(t)-P \sin (\alpha)
$$

In the above equation, it can be seen that if the inertial mass effective weight is equal to applied axial load, the equivalent lateral force due to the overturning moment from the inertial mass system and the restoring force of the post-tensioning bar cancel out. Furthermore, since $\alpha$ is typically small, $P^{\prime} \approx P$ and therefore, the proposed inertial mass system reproduces the P- $\Delta$ effects very close to the expected results when the mass is placed directly on top of the column. 


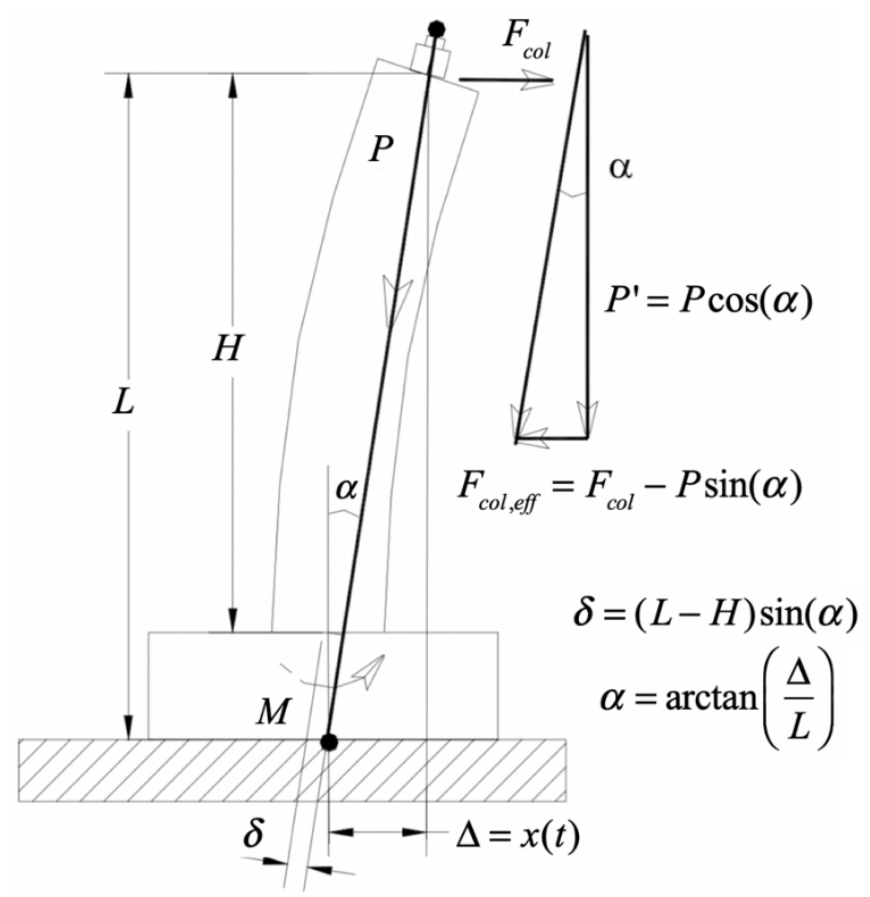

Figure 2.8 P-Delta effect due to axial load

\subsubsection{Calculation of Damping Coefficient}

As it was demonstrated previously, for a measured response history, the P- $\Delta$ force term can be calculated separate from the combined force since all terms involved are known. Nevertheless, the spring and damping force cannot be separated from the measured response since both $k(t)$ and $c$ are unknown.

One way to characterize the damping is through the viscous damping ratio. Because it is not possible to determine analytically the damping ratio $\zeta$ for practical structures, for reinforced concrete columns can be calculated from experimental testing. Free vibrations experiments provide one mean of determining the damping using the log decrement method. The damping ratio $\zeta$ is determined from the definition of the logarithmic decrement: 


$$
\delta=\ln \left(\frac{u_{i}}{u_{i+j}}\right)=\frac{2 \pi j \zeta}{\sqrt{1-\zeta^{2}}}
$$

Where $u$ and $u_{i+j}$ are the peak values of force, displacement or acceleration on the first and $j^{\text {th }}$ successive cycle respectively.

\subsection{Numerical Comparison to Existing Inertial Mass Systems}

As it was discussed previously, different inertial mass system configurations have been used in shake table testing of structures. Of these configurations, two are selected to be compared numerically to the proposed inertial mass system. One setup, which represents the most realistic option for assessing the performance of structural components under earthquake loading, considers placing the additional inertial mass directly on top of the specimen. The second configuration, known as mass-rig, considers putting the extra mass on a supporting structure located outside the shake table platform. In the following, the three systems are compared through refined numerical models using OpenSees (2013). For that purpose, the numerical model of the proposed IMS was developed and calibrated against the experimental results, although this calibration is discussed and presented in details in Chapter 4. The parameters used in this numerical model were then used to develop the numerical models for the other two systems. From now on, Model I refers to the numerical model for the proposed system, Model II to the system with the mass on top of the specimen, and Model III relates to the Mass-rig system. OpenSees was utilized for the numerical simulations because it is widely used in the earthquake engineering research community and has a vast catalog of material 
models, elements, and solution algorithms to model and analyze the nonlinear response of systems.

\subsubsection{Numerical Modelling of Inertial Mass Systems}

In order to characterize the behavior of RC column, two modeling methods have been widely used, namely, lumped-plasticity and distributed-plasticity (Taucer et al., 1991), (Scott \& Fenves, 2006), (Berry \& Eberhard, 2008). In the lumped-plasticity approach, the inelastic behavior of a studied specimen is specified to occur only at specific regions of the element where the plastic deformations are important (plastic hinges). Outside of the plastic hinge regions, the element behaves as linear elastic. The length of the plastic hinge region is specified by the user and modeled using fibers with two integration points, one at each end of the plastic hinge. The properties of the elastic portion of the element can be specified in such a way that the initial stiffness is adequately modeled. In the distributed-plasticity approach, the nonlinear behavior of a studied specimen is modeled using nonlinear beam-column elements discretized using fiber sections and the total length of an element is divided into a number of segments or integration points. This method offers a more accurate description of the inelastic behavior, since they allow inelastic deformations to be developed anywhere within the member. It is worth mentioning that the nonlinear beam-column element does not account for shear deformations or bond-slip rotations; however, these effects can be added to the RC element using springs.

To contrast the difference in response of the three inertial mass system, an RC bridge column model was considered in the modeling. The specimen model was part of 
the experimental program on large-scale circular cross-section reinforced concrete bridge columns. Details of the experimental program are presented and discussed in Chapter 3 . The cross-sectional dimension and reinforcement of the column model considered as part of the study are depicted in Figure 2.9. The RC column is a scale model of a typical pre1970 circular RC column part of a multi-column bent in the State of Oregon, USA. Such columns are commonly seismically deficient according to current seismic provision, given the poor confinement provided at zones where large inelastic incursions are expected. For the purpose of comparison, constant axial load $0.09 f_{c}^{\prime} A_{g}=75$ kips was considered for the analysis.

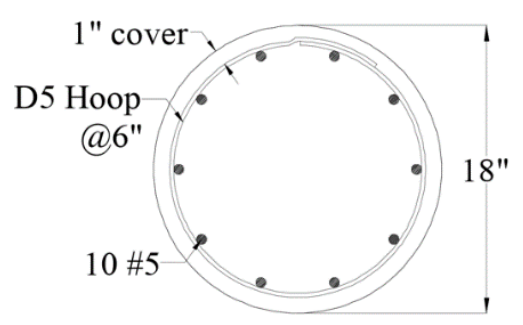

Figure 2.9 Cross section of RC column model

Nonlinear Time History Analysis was performed in OpenSees (2013) for comparison of the inertial systems. The input ground motion for the analysis was selected from the Maule, Chile M8.8 earthquake in an effort to simulate a potential Cascadia Subduction Zone event. With that purpose, the transverse direction of the ground motion recorded at the Curico station (Curico X) was considered as an input for the numerical model. The acceleration record was amplified using a factor equal to 1.57 , as it was considered in the experimental program. Details of the scale factor used are kept for Chapter 3 since this section aims to compare the performance of the different inertial 
mass systems. Figure 2.10 depicts the original acceleration and integrated displacement history of the Curico record used for the NLTHA.

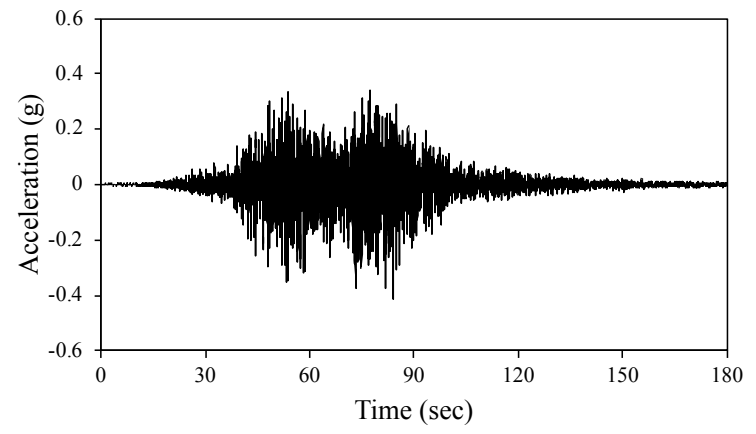

a)

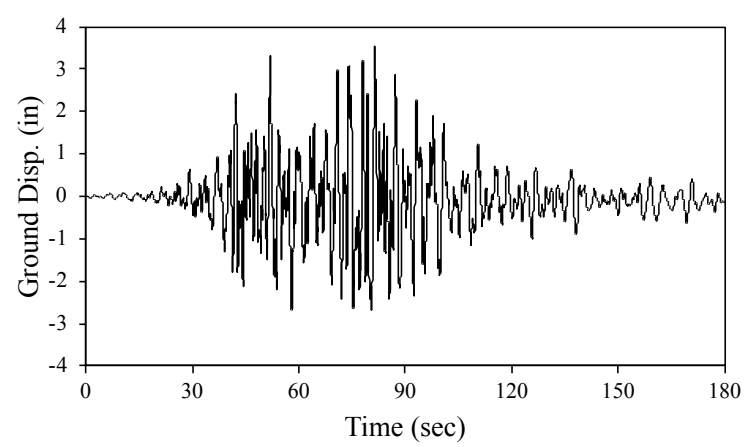

b)

Figure 2.10 Time histories for Curico X record. a) Acceleration, b) Displacement

\subsubsection{OpenSees Model I}

As it was mentioned before, the IMS was part of the experimental program that is presented in Chapter 3 and depicted in Figure 2.4. In general, the test setup includes the IMS, an RC column, axial load system, and the shake table system. The numerical model of the setup used in this study is illustrated in Figure 2.11. The RC column was modeled using the fiber-based distributed-plasticity model formulation proposed by Taucer et al. (1991). The modeling strategy was calibrated with experimental results that are presented in Chapter 3. In the chosen modeling strategy, a force-based fiber beam-column element, a zero-length bond section, and an elastic shear component are combined to model the flexural, bond-slip, and shear components of the total column deflection. The force-based beam-column element with five integration points was used to represent the column, following the recommendation of Berry and Eberhard (2008). Because most nonlinear behavior occurs near the base of the column, a Gauss-Lobatto integration scheme was used as the plastic hinge integration method. 


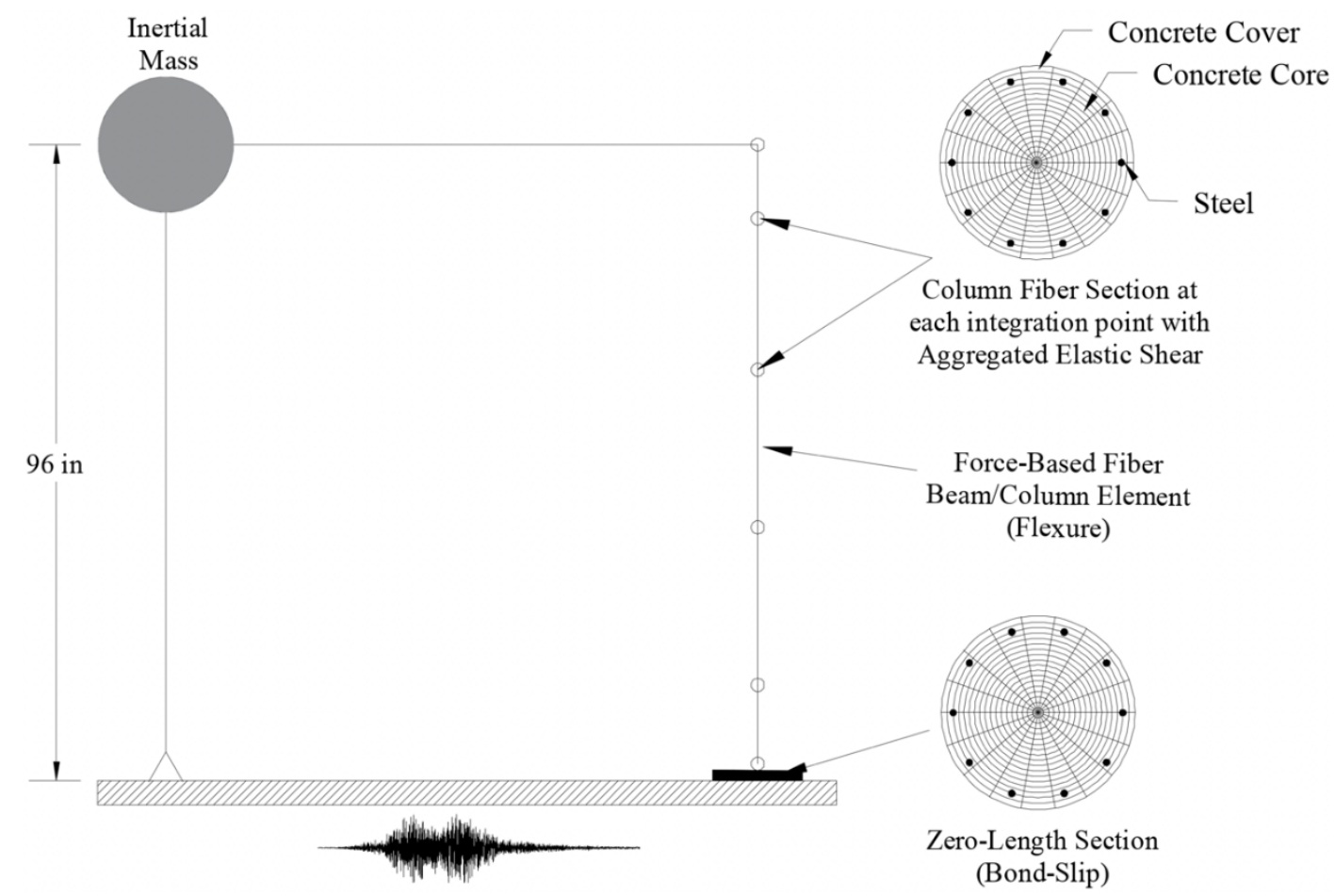

Figure 2.11 OpenSees Numerical Model I for the proposed IMS

The column section was divided into three fiber sections: confined concrete core, unconfined concrete cover and reinforcing steel. A radial discretization scheme was used to discretize the cross-section, and it was as follow: 16 radial core divisions, 18 transverse core divisions, 2 radial unconfined cover division, and 18 transverse cover division. The core concrete, cover concrete, and longitudinal steel fibers each have a uniaxial stressstrain model associated with them. The Popovic's curve with degraded linear unloading/reloading stiffness and tensile strength with exponential decay and model parameters proposed by Mander et al. (1988) (Concrete04) was used to model both the confined and unconfined concrete. The longitudinal reinforcing steel was modeled using the uniaxial material model Hysteretic Material. This material model was chosen because its capabilities for capturing pinching of force and deformation, as well as damage due to 
ductility and energy, and degraded unloading stiffness that can be caused by concrete crushing, splitting cracks, bar buckling and bar fracture. The parameter associated to the pinching behavior, pinchx and pinchy, were both set equal to 1.0 based on the observed pinching behavior in the test specimens. The damage parameter associated to damage due to ductility, i.e., parameter damage1, was set equal to 0.008 to account for the cyclic deterioration from one cycle to the next cycle observed in the test specimens. The damage parameter associated to damage due to energy, i.e. parameter damage2, was set equal to 0.004 in an effort to capture the strength deterioration observed in the test specimens. The degraded unloading stiffness parameter (beta) was set equal to 0.3 . In order to improve the prediction of column deformation, shear and bond-slip behavior must be included into the model. Shear deformations are neglected with a standard fiber beam-column element formulation, which only provides the flexural behavior. Additional flexibility from shear behavior was added to the cross sections at the column-footing interface using a section aggregator. The shear behavior was idealized as an isotropic material with constant shear modulus, $G_{\text {eff }}$, equal to $0.2 \mathrm{E}_{\mathrm{c}}$. This value was chosen following the recommendation of Elwood and Eberhard (2009). As with shear behavior, the standard fiber beam-column element formulation neglects the additional flexibility coming from the slip of the longitudinal reinforcement at the anchorage, because it assumes a perfect bond between the concrete and steel. To model the bond-slip behavior, the model proposed by Ghannoum (2007) and the recommendations of Mehary et al. (2018) were used. In this bond-slip model, the slip behavior is modeled using a zero-length fiber section with the same discretization scheme used for the force-based 
beam-column element. However, this model replaces the stress-strain relationships in the zero-length section by an equivalent stress-slip relationship for the steel and concrete fibers as shown in Figure 2.12 and Figure 2.13, respectively. The slip displacement at yield $\left(s_{y}\right)$ in the steel fibers can be obtained from the measurements of the LVDTs located at the base of the columns. For the purpose of comparing different inertial mass systems, a value of 0.03 in. for the slip displacement at yield was used in the distributedplasticity model. The steel fibers in the bond-slip zero-length element were modeled using the Steel02 Giuffre-Menegotto-Pinto model with isotropic hardening because its easy implementation. Uniaxial concrete material model Concrete 04 was used for the confined and unconfined concrete in the bond-slip zero-length element. To avoid discontinuities in the steel stresses and neutral axis location between the fibers of the nonlinear beam/column element and the bond-slip section (Ghannoum, 2007), the strains in this section were also modified. This modification was accomplished by multiplying the concrete strains by a scale factor, $\mathrm{SF}_{\mathrm{conc}}=\mathrm{sy} / \varepsilon_{\mathrm{y}}$. Even though this scale factor is dimensionally incorrect, it allows an increase in the concrete strains to maintain compatibility between the fiber sections. Scale factor of 17 was used for the analysis. In order to represent the inertial forces transferred to the specimen, the IMS was modeled in OpenSees using an equivalent elastic beam-column element with pin support and total height equal to the vertical distance from the pin at the base of the IMS to the longitudinal axis of the rigid link (i.e., 96 in). Additionally, a truss element was used to represent the rigid link connecting the IMS and the specimen. The option of Corotational 
transformation was assigned to the beam-column representing the IMS to capture P-delta effects.

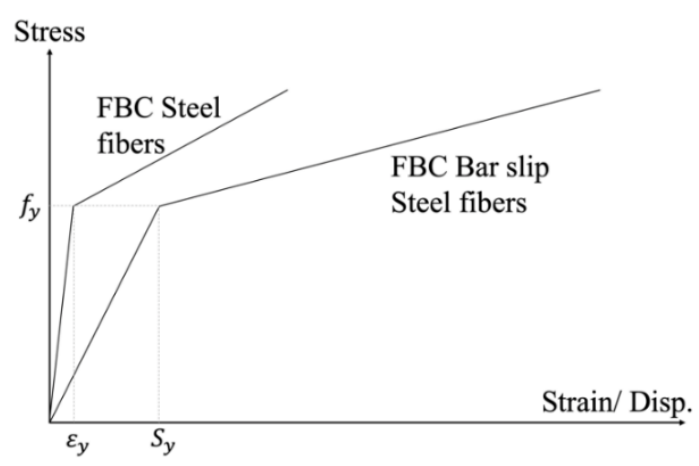

Figure 2.12 Steel fiber stress-strain relations

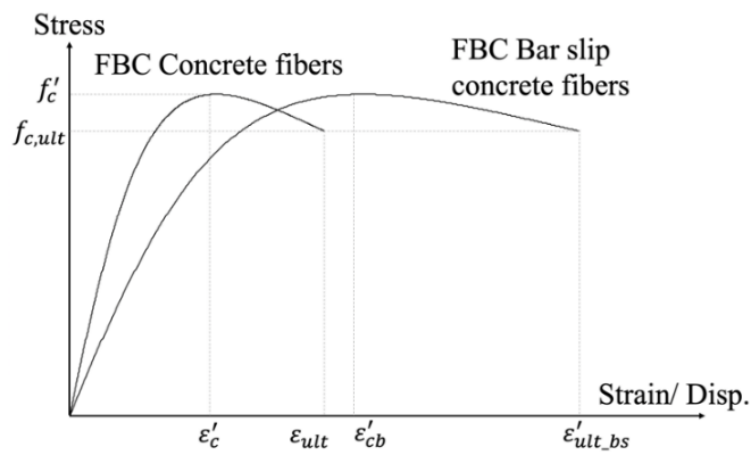

Figure 2.13 Concrete fiber stress-strain relations

\subsubsection{OpenSees Model II}

One of the mass setups that has been used for shake table tests is to connect and place the additional inertial mass directly on top of the column. This configuration is the most realistic option for mimicking the behavior of single column RC bridge bent, and for that reason, the proposed IMS is compared to it. The numerical model for this setup is illustrated in Figure 2.14. The same numerical modeling strategy used for the IMS and described previously was used in the OpenSees model II. The effective inertial mass of the IMS, including half of the column weight, was lumped on top of the column model 
according to the values presented in Table 2.1. Also, the option of Corotational transformation was assigned to the force beam-column element representing the $\mathrm{RC}$ column to capture the P-delta effects.

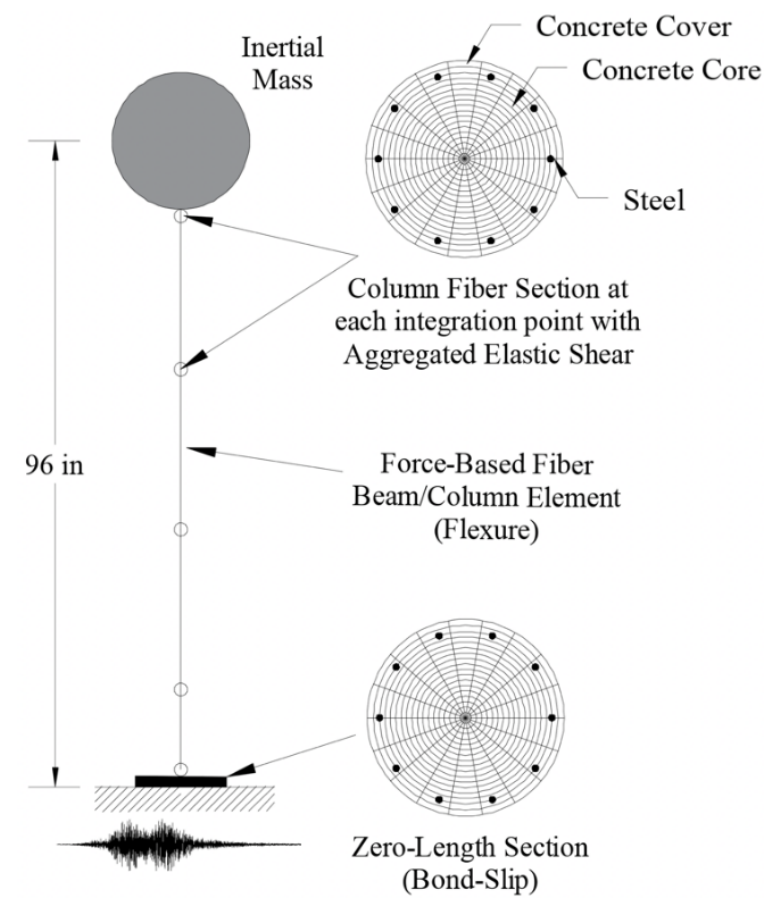

Figure 2.14 OpenSees Numerical Model II

\subsubsection{OpenSees Model III}

The third inertial mass system considered to be compared to the OpenSees Model I is commonly termed as mass-rig. In this setup, the additional inertial mass for the shake table experiments is placed in a structure located outside the shaking platform. Therefore, only the column specimen is subjected to dynamic loading. This configuration was proposed by Laplace et al. (1999) and it was described in a previous section. To create OpenSees Model III, minor changes must be introduced to OpenSees Model I to replicate the mass-rig system, but these modifications do not affect the column modeling strategy used. One of the modifications consist of applying the earthquake loading only to the 
column model, because in the test setup proposed by Laplace and shown in Figure 2.3, only the column model is attached on the shaking platform. The second change is related to the input time history. OpenSees allows applying the ground motion history to specific nodes in the model employing the command Multi-Support Excitation Pattern, which applies a displacement ground motion. When using this command, the input motion can be acceleration or displacement time history. If using the acceleration record as input, OpenSees automatically integrates the record to obtain the displacement record. However, it is recommended that the user input the displacement since the integration method used by the program it is not accurate. In OpenSees model III, the displacement time history of the Curico X record shown in Figure 2.10b) was used.

It is worth noting that according to Laplace (1999), besides the inertial forces induced by the total mass on the mass-rig, significant lateral forces were transmitted to the specimens due to secondary moments (P-delta effects) on the mass-rig. These additional lateral forces resulted from the overturning moment of the system, which is equivalent to the vertical force times the lateral drift. As it was also pointed out by Laplace, no considerable secondary moments were generated in the specimen column due to the axial force system. As in OpenSees model I, to represent the additional forces transferred to the column, the inertial mass system was modeled using an elastic beamcolumn element with a pin support and a total vertical distance pin-to-pin of the IMS (96 in.). Also, P-delta effects in the IMS were considered using the option of Corotational geometric transformation. Figure 2.15 illustrates the analytical model used for unidirectional tested specimens. 


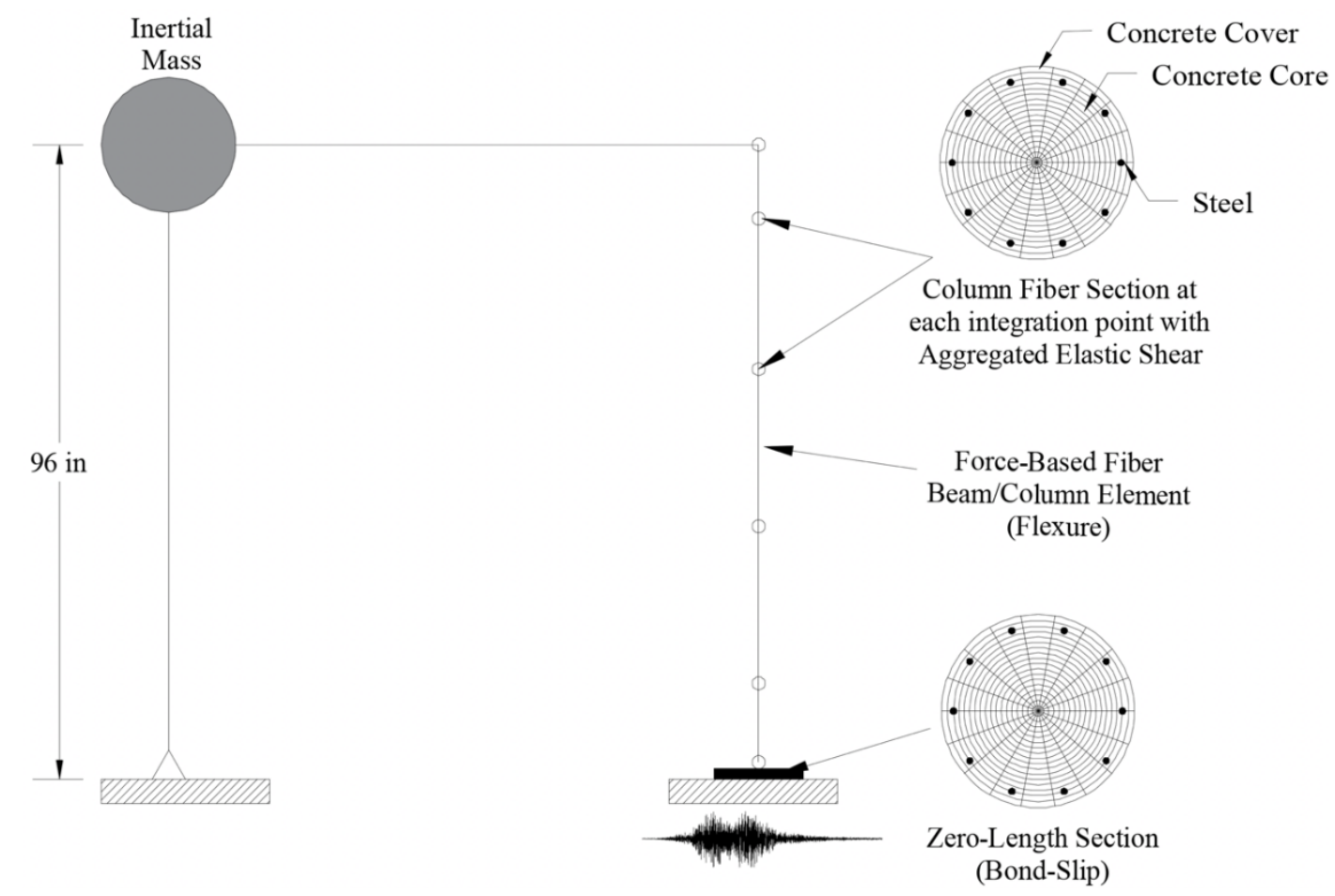

Figure 2.15 OpenSees Numerical Model III

\subsubsection{Comparison and Discussion}

Comparisons between the proposed IMS and the other two inertial mass system using the distributed-plasticity approach are presented in this section. Figure 2.16 and Figure 2.17 illustrate the calculated seismic performance in terms of displacement histories and force-displacement hysteresis for each numerical model. Additionally, the experimental hysteresis curve used for calibration is presented. From the results, it is noted that in general the OpenSees model I replicate the initial stiffness, strength and displacement capacities, pinching effect, a strength and stiffness degradation of the tested specimen reasonably well. Then, using the same calibrated parameters in the OpenSees model II, it is seen that the proposed IMS closely matched the calculated performance of OpenSees model II, which represents the ideal scenario for testing cantilever bridge 
columns in a shake table, i.e., place the mass on top of the specimen. On the other hand, by applying the conditions imposed by the mass-rig setup (Laplace et al., 1999) to the proposed IMS, the OpenSees model III presents deficiencies capturing the strength capacity of the tested specimen. Also, the displacement capacity and pinching effect are not well captured.
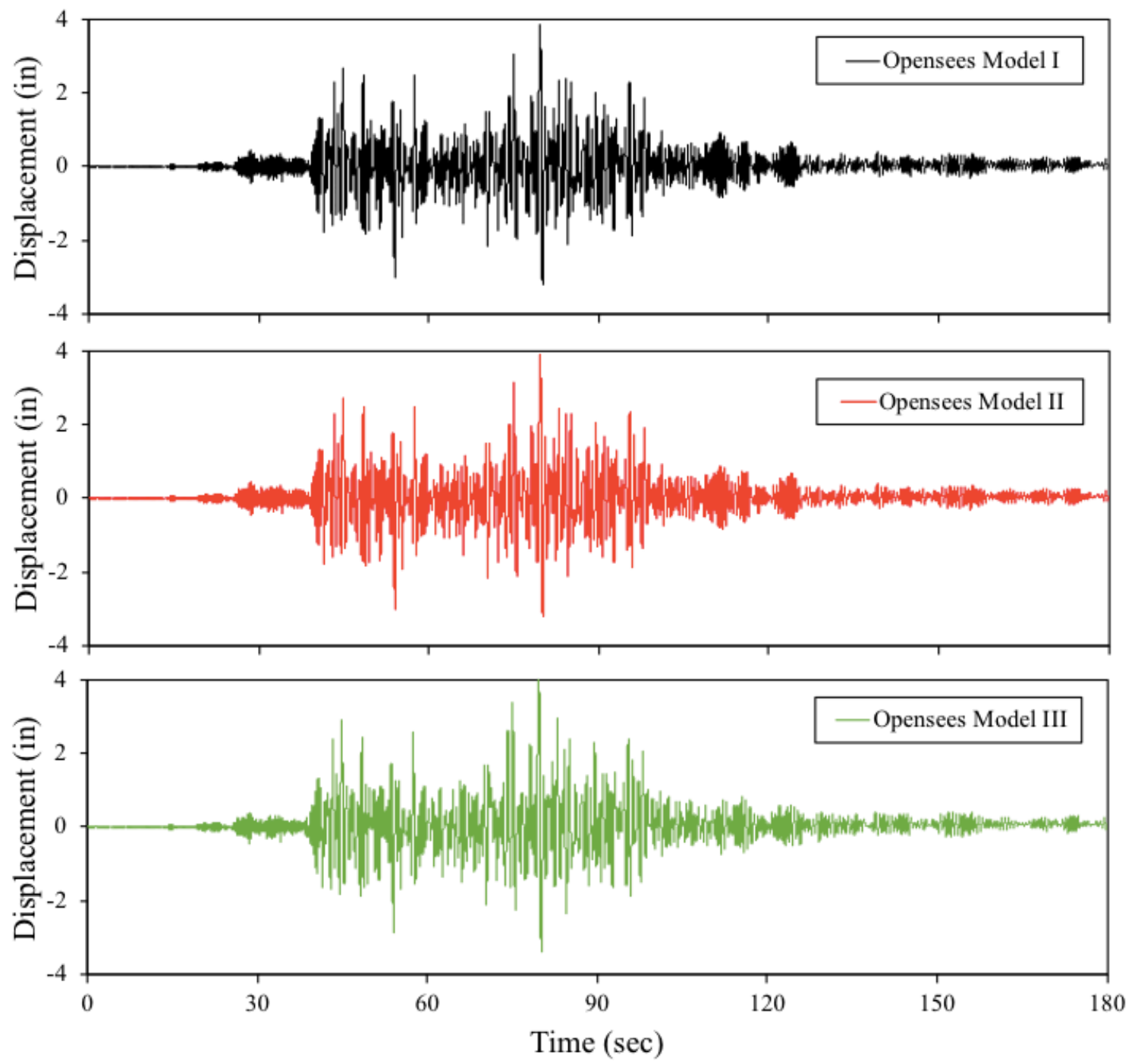

Figure 2.16 Column displacement history comparison 


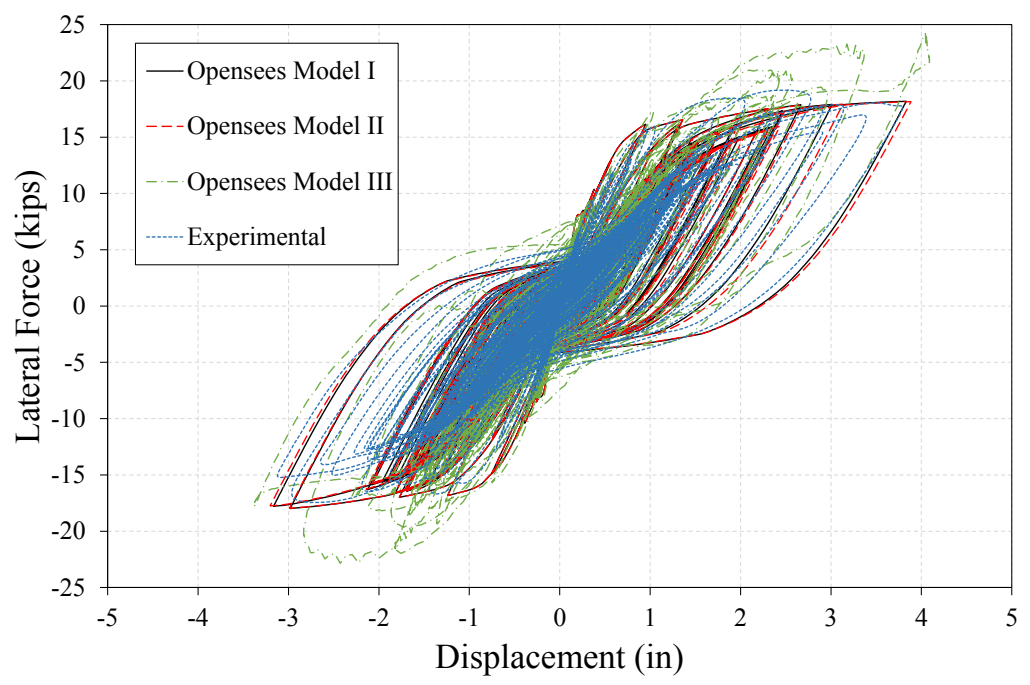

Figure 2.17 Force-displacement hysteresis comparison

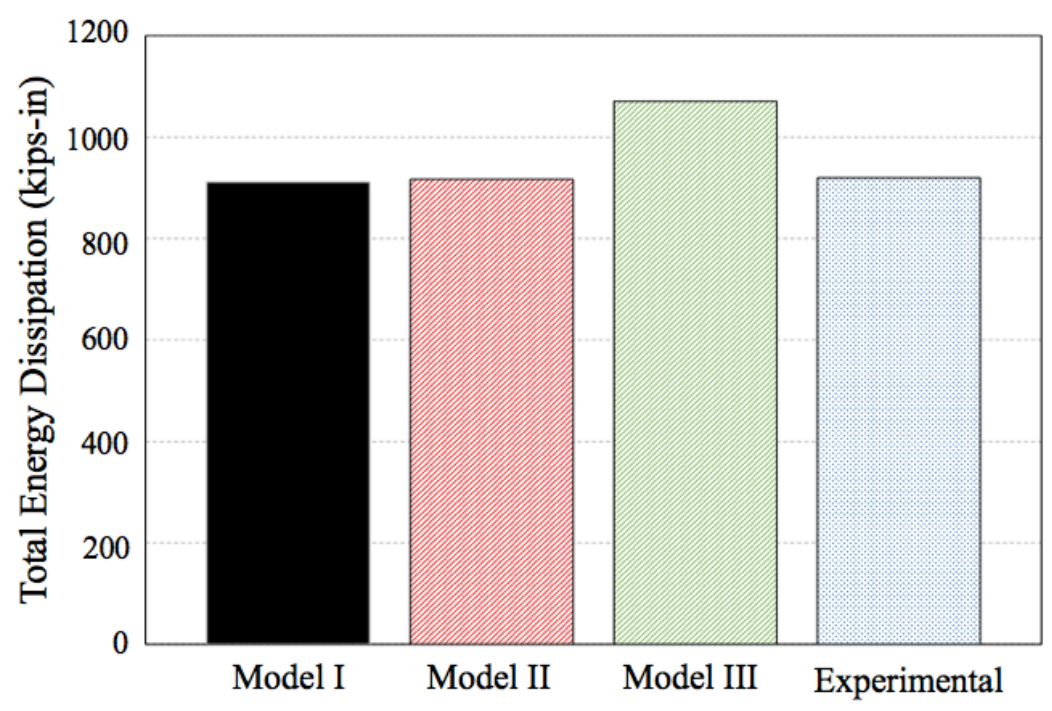

Figure 2.18 Energy dissipation comparison

Figure 2.18 depicts the total dissipated energy in the experiment compared to the ones calculated for the numerical models. The total energy dissipated was calculated adding the enclosed area of each cycle in the force-displacement hysteresis curve. The OpenSees model III tend to overestimate the total energy dissipated by the specimen by about $16 \%$ with respect to the experimental value. This overestimation is primarily 
caused by the secondary moments (P-delta effects) that the mass-rig transferred to the column. On the contrary, OpenSees model I and II present a negligible overestimation in the total dissipated energy as compared to the experimental results, with an error of $0.87 \%$ and $0.31 \%$, respectively.

Based on these results and the advantages identified (safety for attaining high performance levels, small out-of-plane displacements, reduced time for test preparation and proper reproduction of P-delta effects), led to the conclusion that the proposed IMS can be a suitable inertial mass system for dynamic testing using shake table experiments. 


\section{CHAPTER 3. Large-Scale Shake Table Experiments of Substandard RC Bridge Columns}

\subsection{Introduction}

The experimental program at Portland State University was focused on studying the seismic performance of substandard reinforced concrete bridge columns subjected to real-time subduction-zone earthquake motions. For this purpose, six 1/2-scale single columns, designated as C1C-L, C2C-M, C3C-T, C4S1-L, C5S1-M, and C6S2-M, were subjected to excitations on a shake table system. Description of the nomenclature adopted is given in the following sections. A new inertial mass system (IMS) was developed as part of the study to test on shake table single cantilever-type columns as presented and discussed in Chapter 2.

This chapter discusses the design and experimental setup of the six test columns. Details of construction, material tests, instrumentation, shake table test setup, and testing program are presented. Besides, this chapter presents the results of the shake table tests of the six specimens, where the progression of testing and damage sequence is demonstrated. The global behavior of the columns, regarding displacements, hysteresis, stiffness, and changes in the natural period are discussed. The local response in terms of strains in the reinforcement and section curvatures is also presented. 


\subsection{Model Selection}

Two sets of substandard cantilever RC bridge columns were constructed to represent a column that would normally be part of a seismically deficient multi-column bent constructed in the 1950 to 1970 in the State of Oregon, USA, as shown in Figure 3.1(a). As many of the bridge structures built at that time in the Pacific Northwest, the bridge substructure was designed and built with minimum seismic considerations. This resulted in inadequate transverse reinforcement, no confinement, no seismic detailing, and lap-splices in the plastic hinge zone of the bent. The representative bridge bent consists of two circular columns per bent, a rectangular cap beam and rectangular pile cap footings. The column longitudinal reinforcement ratio is $\rho_{\mathrm{L}}=1.2 \%$, which is barely above the minimum required by AASHTO $\left(\rho_{\mathrm{L}}>1 \%\right)$. Conversely, the column deficiencies are vast. The provided column shear reinforcement $\left(\rho_{\mathrm{s}}=0.2 \%\right)$ does not meet the code requirement $\left(\rho_{\mathrm{s}}>0.5 \%\right)$. The column confinement is almost non-existent since \#4 circular hoops spaced at 12" were provided. Moreover, lap splices can be found in expected plastic hinge zones and no seismic detailing was specified. The specified material properties were 3300 psi as compressive strength of concrete at 28 days and Grade 40 steel.

The specimen's cross-section for this research was identical to the columns' cross-section that were part of the $1 / 2$-scale $\mathrm{RC}$ bridge bent of the representative bridge (Figure 3.1(a)) tested in a previous study by Bazaez (2017), as shown in Figure 3.1(b). All specimens consisted of a circular cross-section of 18 in diameter. In order to calculate the height of the specimens, a target displacement ductility demand of 4 and a target 
period of vibration of the column around $0.5 \mathrm{sec}$ were first calculated in an iterative process. The column height was varied while the longitudinal steel, material properties, axial load, and the cross-section dimension were kept constant. The target displacement

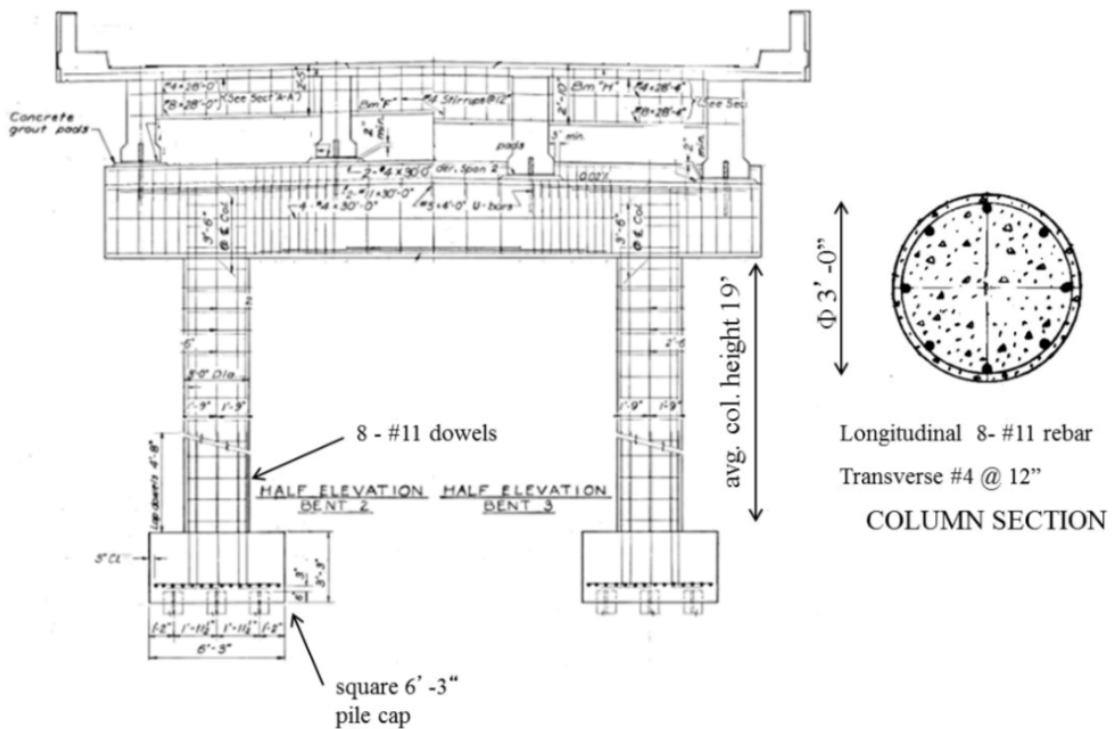

a) Prototype

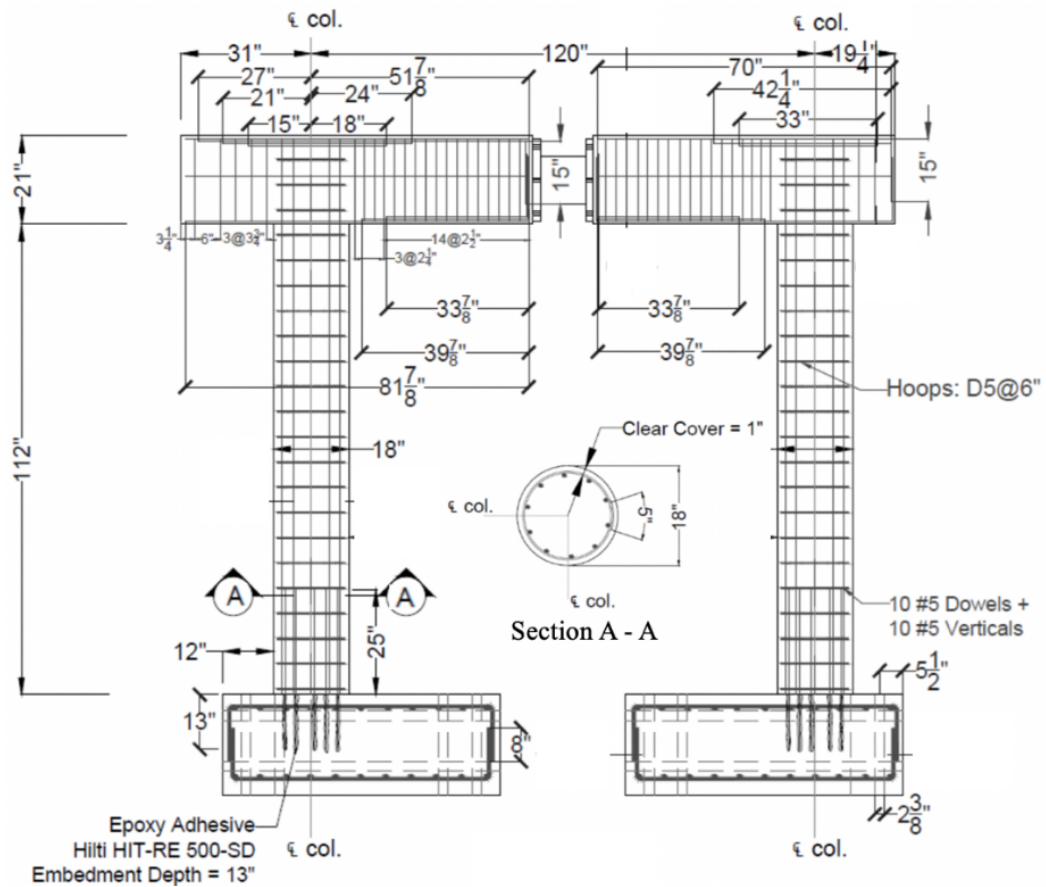

b) $\mathrm{RC}$ bridge bent specimen

Figure 3.1 Representative multi-column RC bridge bent (Bazaez, 2017) 
ductility was defined as the ratio of the displacement demand for a 1000-year event and the idealized effective yield displacement of the column at the formation of the plastic hinge, as per Eq. (2.1). The displacement demand was obtained from the representative design response spectrum (Bazaez, 2017) depicted in Figure 3.2, and calculated using Eq. (2.2). This response spectrum corresponds to a 1000 -year event ( 7 percent probability of exceedance in 75 years) and aims to be representative of a vast number of prestressed concrete stringer/girder bridges in Oregon. Further, the displacements were magnified through the use of the displacement magnification factor for short period structures, $R_{d}$ as per AASHTO (2009), and presented in Eq. (2.3).

$$
\begin{gathered}
\mu_{D}^{c o l}=\frac{\Delta_{D, 1000}}{\Delta_{y}^{c o l}}=\frac{\Delta_{D, 1000}}{\frac{H^{2}}{3} \cdot \phi_{y}} \\
\Delta_{D, 1000}=R_{d} \cdot \frac{S_{a, 1000} \cdot T_{n, \text { crack }}^{2} \cdot g}{4 \pi^{2}}
\end{gathered}
$$

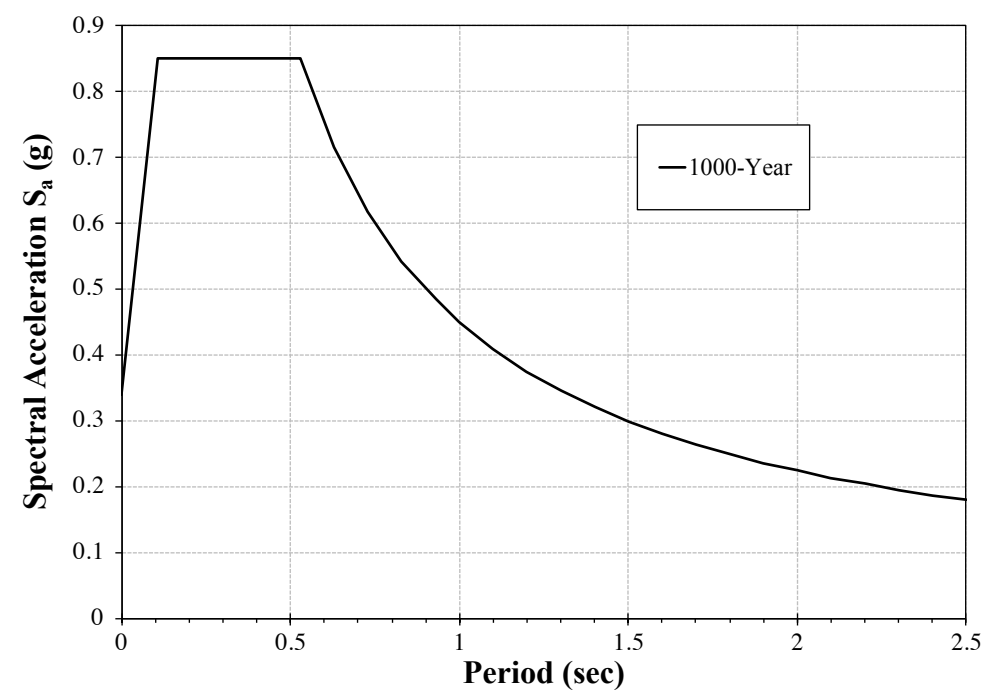

Figure 3.2 Representative response spectrum 


$$
R_{d}=\left\{\begin{array}{rrr}
\left(1-\frac{1}{\mu_{D}}\right) \frac{1.25 T_{s}}{T_{e}}+\frac{1}{\mu_{D}} \geq 1.0 & \text { for } & \frac{1.25 T_{s}}{T_{e}}>1.0 \\
1.0 & \text { for } & \frac{1.25 T_{s}}{T_{e}} \leq 1.0
\end{array}\right.
$$

Where, $T_{n, \text { crack }}$ is the fundamental period of the SDOF system; $S_{a, 1000}$ is the spectral acceleration given by the 1000-year event response spectrum; $\mathrm{g}$ is the standard gravity constant; $\mathrm{H}$ is the distance from the point of maximum moment to the point of contra-flexure, and equal to the column height for a cantilever column; $\phi_{y}$ is the idealized yield curvature defined by an elasto-plastic representation of the cross-section $M-\phi$ curve; $T_{s}$ is the period at the end of constant design spectral acceleration plateau, and $\mu_{D}$ is the maximum local member displacement ductility demand. AASHTO (2009) states that $\mu_{D}$ may be taken as 6 . The $M-\phi$ analysis was performed using the expected material properties, which were determined by applying factors to the specified properties of the representative bridge. Factors of 1.3-1.5 and 1.1 are recommended in the literature for the compressive strength of concrete and the yield stress of steel, respectively (Priestley et al., 1996), (AASHTO, 2009). The axial load was assumed to be $10 \%$ of the column axial capacity $\left(0.10 f_{c}^{\prime} A_{g}\right)$. Idealized yield curvature $\phi_{y}$ and cracked moment of inertia $I_{\text {crack }}$ were then obtained. Then, the fundamental period of the column was calculated using Eq.(2.4), where a seismic weight of 42.5 kips was considered. Finally, based on the results shown in Figure 3.3 and also dictated by the laboratory constraints, a column height of $8 \mathrm{ft}$ was chosen. For this value, a displacement ductility demand of 4 and a fundamental period of $0.44 \mathrm{sec}$ are obtained. 


$$
T_{n, \text { crack }}=\frac{W \cdot 2 \cdot \pi}{3 \cdot 57,000 \cdot \sqrt{f_{c e}^{\prime}} \cdot I_{\text {crack }} \cdot g} \cdot H^{3}
$$

Where, $\mathrm{W}$ is the seismic weight of the SDOF system and $f_{c e}^{\prime}$ is the expected concrete compressive strength.

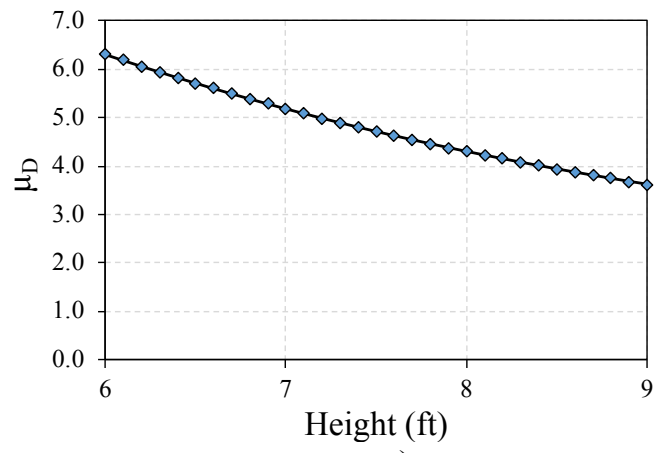

a)

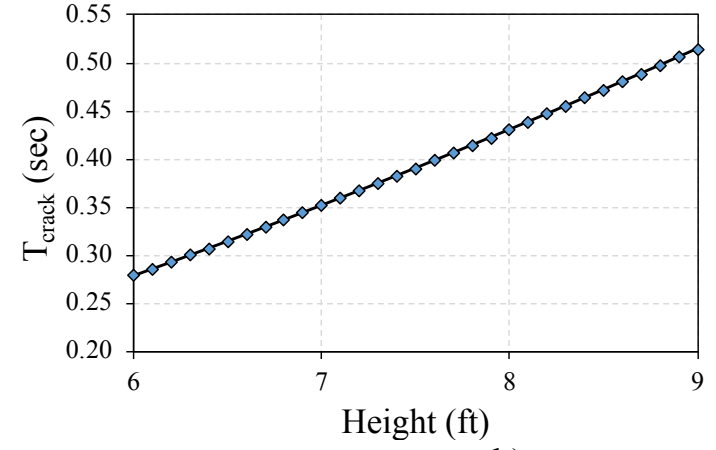

b)

Figure 3.3 Parametric study on column height

\subsection{Specimen Design}

The experimental program consisted of two sets of large-scale of substandard RC bridge columns. The specimens were designed to behave in single curvature (cantilever columns). Two design parameters considered in these sets were the location and length of the lap-spliced longitudinal starter bars in the footing. The first set consisted of three columns, which had the longitudinal steel splices outside the expected plastic hinge zone. The second set also consisted of three columns, but they had the longitudinal steel splices inside the expected plastic hinge zone, in an effort to more closely represent typical columns constructed in the 1950s to mid-1970s in the Pacific Northwest. Thus, two specimens had a lap splice length of $25 \mathrm{~d}_{\mathrm{b}}$, and one specimen had a lap splice length of $40 d_{b}$. Additional details of the test specimens are illustrated in Figure 3.4. 


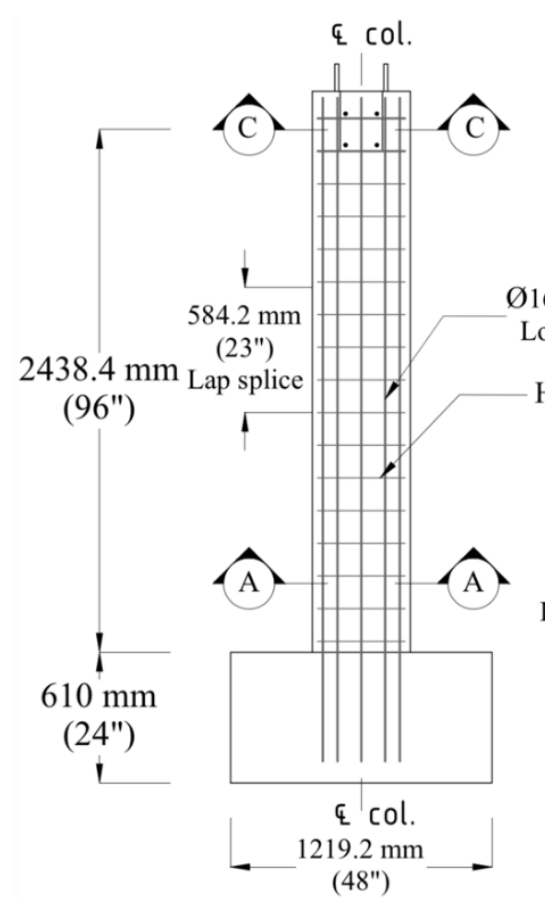

a)

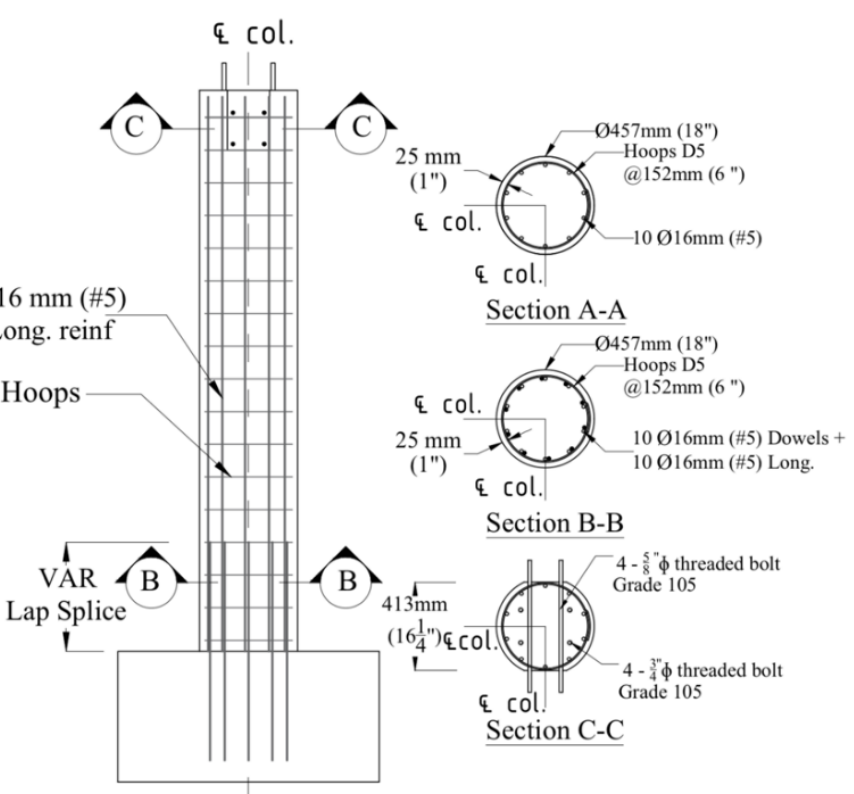

q col. $1219.2 \mathrm{~mm}$

$(48 ")$

b)

Figure 3.4 Specimens Details. a) Continuous reinforcement, b) Lap-spliced

The sequence of construction for the specimens is depicted in Figure 3.5. First, wood forms were set up for the specimen footing, which was designed to contain two columns. The footings were 66 in x 48 in x 24 in, and they were designed to be stiff enough to prevent any footing damage and to fix the cantilever columns. Later, the first set of columns was constructed on one side of the footing, leaving the starter bars of the second set of columns on the other side Figure 3.5(b,c). After the rebar cages for the columns were assembled and strain gauges were installed (more details about instrumentations is presented in the following sections), 18 in. diameter Sonotubes were utilized as formwork and braced to prevent any movement during the concrete pour, as shown in Figure 3.4(d). Once the first set was tested, the second set of columns was built. 


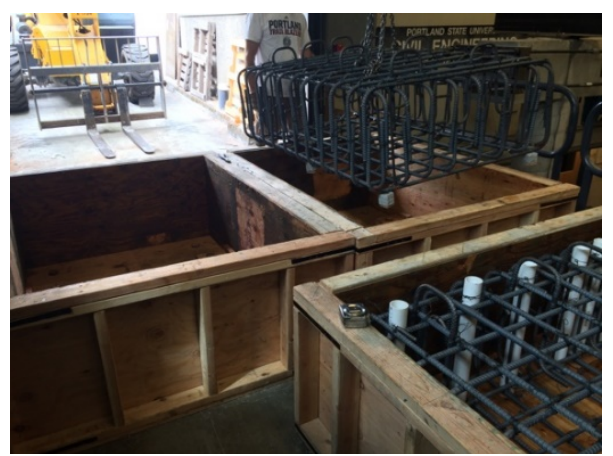

a)

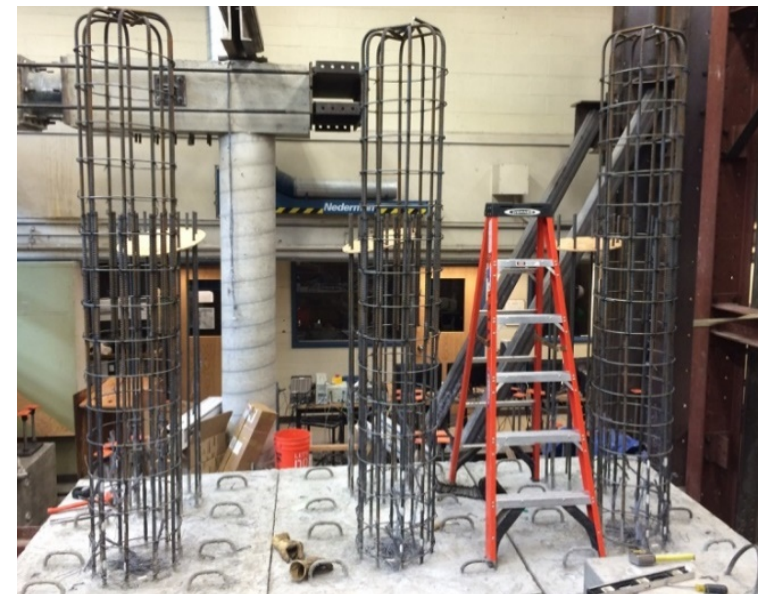

c)

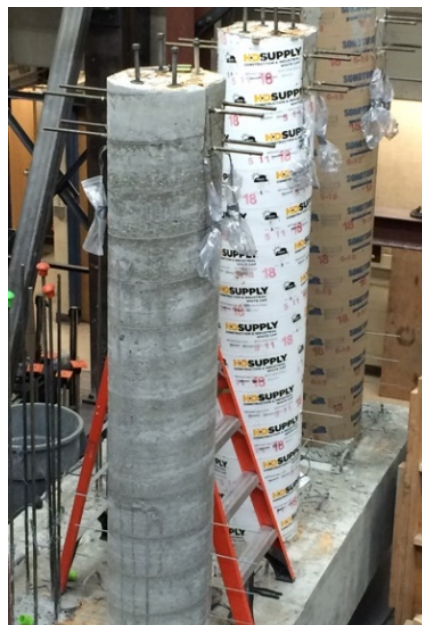

e)

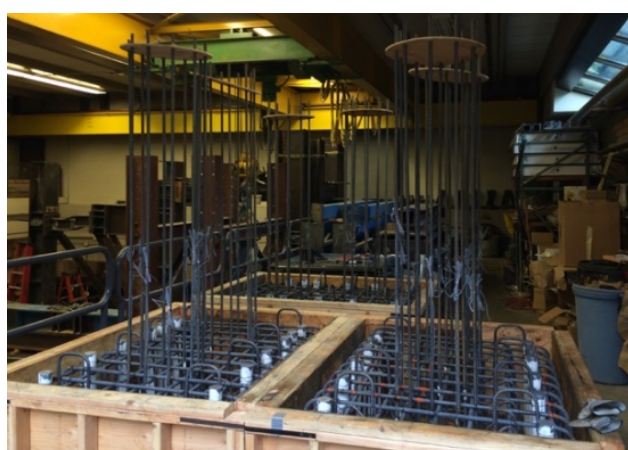

b)

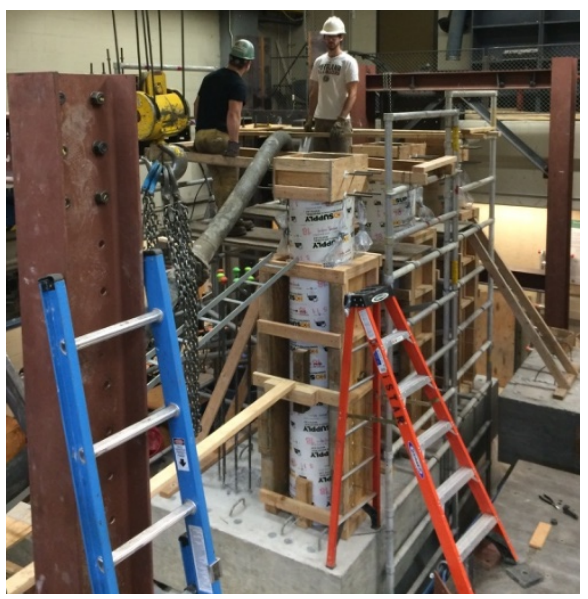

d)

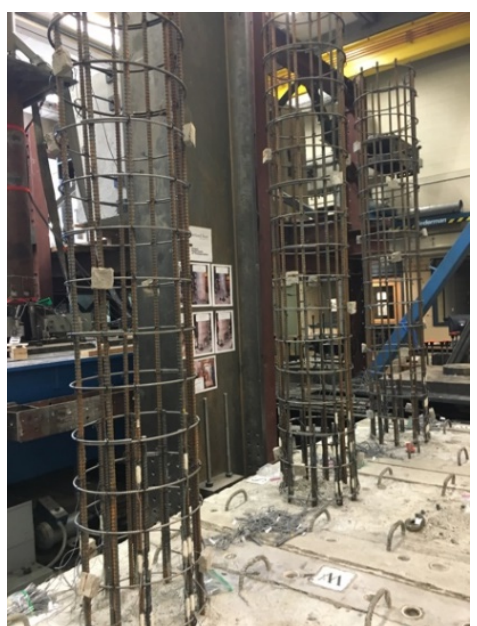

f)

Figure 3.5 Construction sequence for RC bridge columns

The longitudinal column reinforcement consisted of 10 \#5 bars evenly arranged in a circle. All specimens used deformed wire D5 hoops spaced at 6 in on center as 
transverse reinforcement. Grade 40 deformed bars conforming to A615 (ASTM A615, 2018) were used as longitudinal steel in the tested column and D5 deformed wire conforming to A1064 (ASTM A1064, 2017) was utilized as transverse steel. Tensile strengths of the reinforcing steel used in the specimens are summarized in Table 3.1.

Based on construction practices of bridge columns from the era of interest, concrete pouring was performed sequentially. Footings were cast first and then the columns, leaving a cold joint between the pours. The clear concrete cover was 1 in for all columns. Normal weight concrete was used to construct the test specimens with a target 28-day strength of 3300 psi. Standard compression testing of 6 in by 12 in concrete cylinders was performed at 7-day, 28 days and at the day of test completion. The averages of the concrete cylinder tests are also presented in Table 3.1.

Table 3.1 Material Properties

\begin{tabular}{c|c|c|c|c|c}
\hline \multirow{2}{*}{ Specimen } & Concrete & \multicolumn{2}{|c|}{ Longitudinal Reinforcement } & \multicolumn{2}{c}{ Transverse Reinforcement } \\
\cline { 2 - 6 } & $f^{\prime}$ c-me,ksi & $f^{\prime}$-me, ksi & $f_{u-m e}^{\prime}$ ksi & $f^{\prime}$-me, ksi & $f_{u-m e, ~ k s i}^{\prime}$ \\
\hline C1C-L & 4.74 & 51.5 & 79 & 83.6 & 100.4 \\
\hline C2C-M & 4.85 & 51.5 & 79 & 83.6 & 100.4 \\
\hline C3C-T & 4.92 & 51.5 & 79 & 83.6 & 100.4 \\
\hline C4S1-L & 4.27 & 51.5 & 79 & 83.6 & 100.4 \\
\hline C5S1-M & 4.55 & 51.5 & 79 & 83.6 & 100.4 \\
\hline C6S2-M & 4.65 & 51.5 & 79 & 83.6 & 100.4 \\
\hline
\end{tabular}

\subsection{Test Setup}

A schematic representation and the actual test setup are illustrated in Figure 2.4 and Figure 3.6, respectively. The test setup for all test columns was identical and consisted of a cantilever RC column, foundation block, inertial mass, and shake table system. Since the specimens were intended to be dynamically tested using ground motion 
simulation, an inertia mass needed to be connected to the top of the column. Therefore, a new inertial mass system (IMS) was developed for this research, as presented in Chapter 2 and depicted in Figure 3.6.

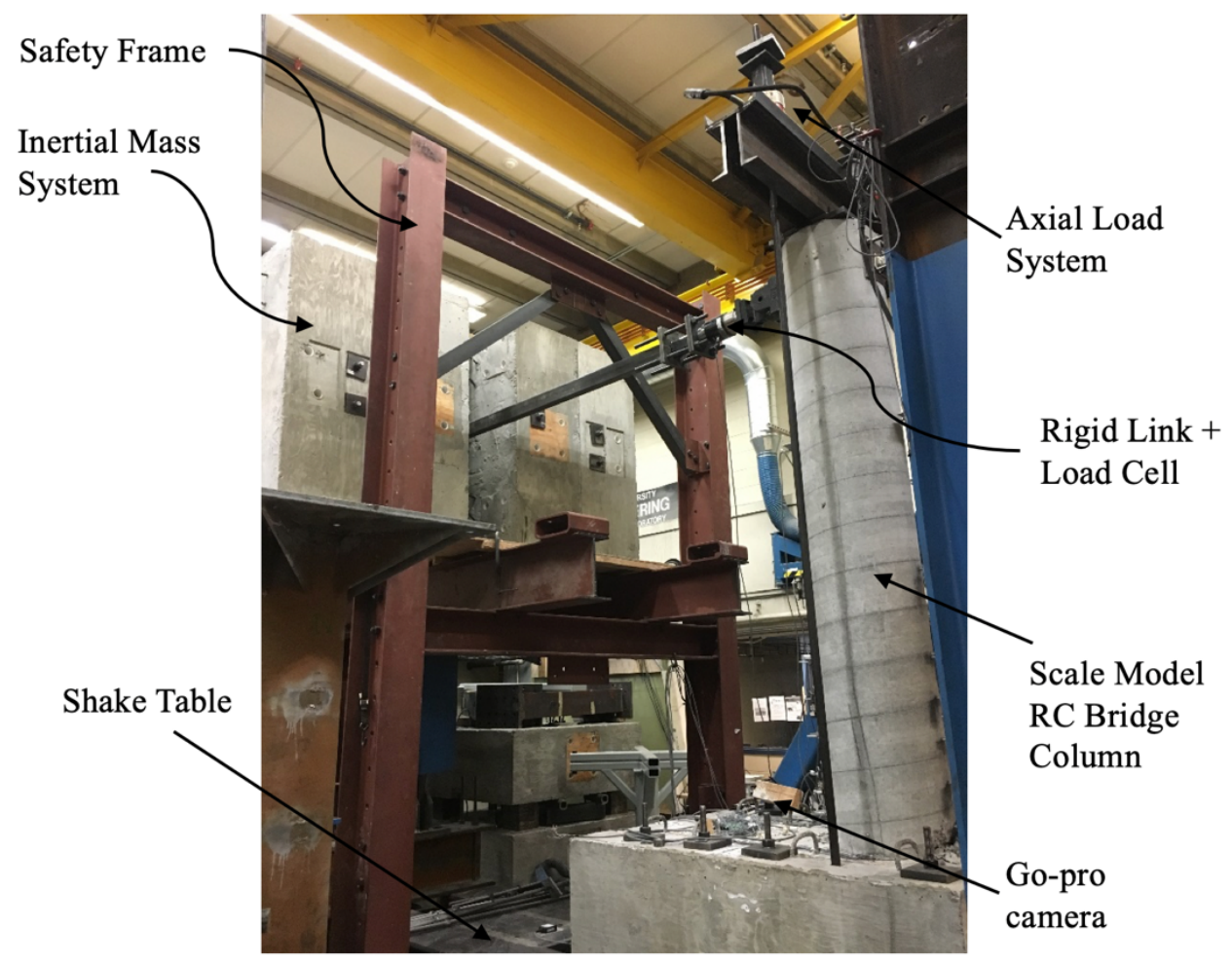

Figure 3.6 Actual test setup configuration

The IMS was explicitly designed for the RC specimens discussed in this study. As can be seen in Figure 3.7, specimens with footing height of 24 in., and a 96 in. height from the column-footing interface to the links were initially tested using the new inertial mass system. Nevertheless, some minor modifications on the specimen dimensions or the IMS could allow the use of higher specimens. Finally, four concrete blocks with a mass of $99 \mathrm{lb}-\mathrm{s}^{2} /$ in were used on the inertial mass system. The steel and RC columns also provide a small portion of the inertial mass, as does the swiveled link system, column axial load system, and the steel beam. Therefore, the total lateral effective inertia mass 
used for this study was $110 \mathrm{lb}-\mathrm{s}^{2} / \mathrm{in}$. This lateral effective mass induced inertial forces that were transmitted to the specimen through a pinned-end rigid link, as shown in Figure 3.8. The axial force was applied through two high-strength rods and hydraulic rams. The axial force was transmitted to the specimen through a steel spreader beam bolted to allthread rods embedded at the top of the column. Axial load indexes typically vary from $5 \%$ to $25 \%$ for bridge columns. Particularly in this study and due to laboratory constraints, $9 \%$ of the nominal column axial capacity $\left(0.09 f_{c}^{\prime} A_{g}=75 \mathrm{kips}\right)$ was used. An accumulator connected to the rams was used to minimize the axial force fluctuations. The actual axial load system is shown in Figure 3.9.

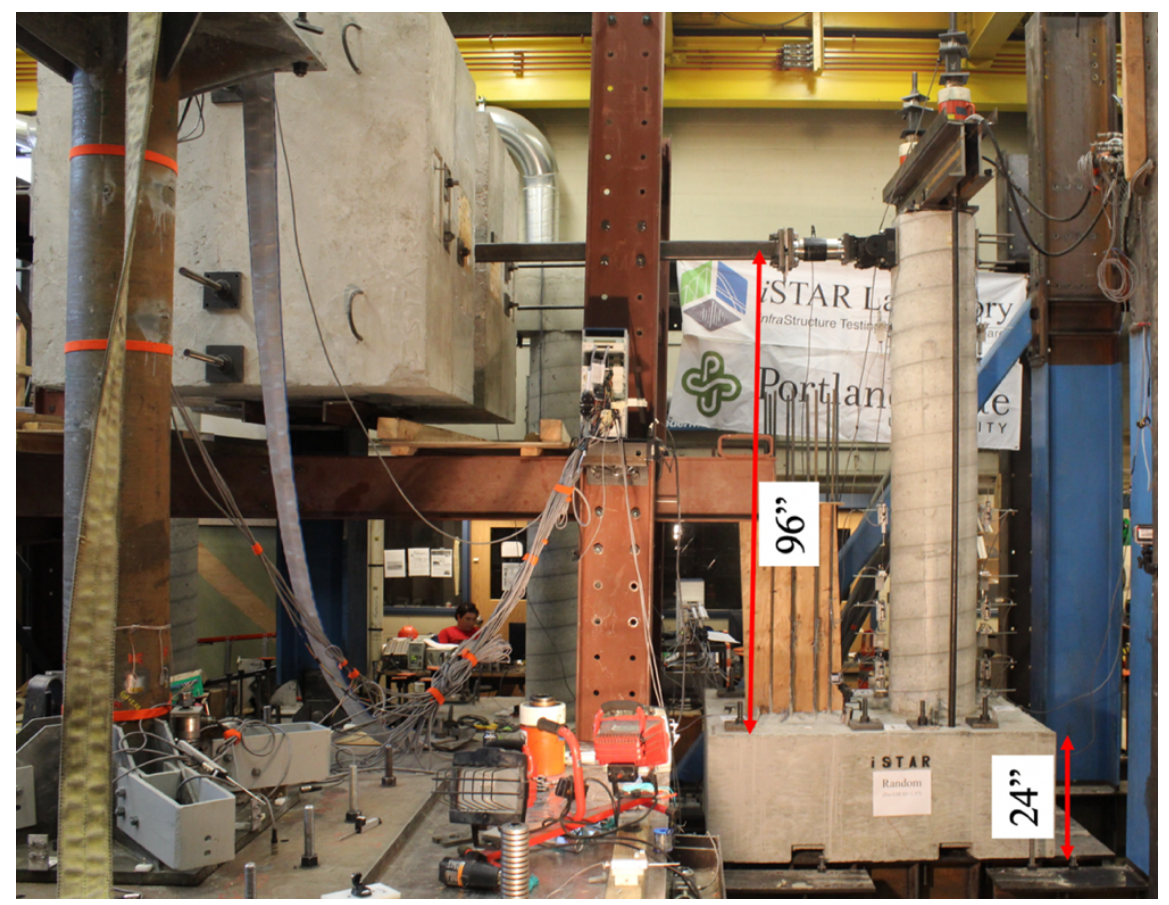

Figure 3.7 IMS and Specimen before testing 


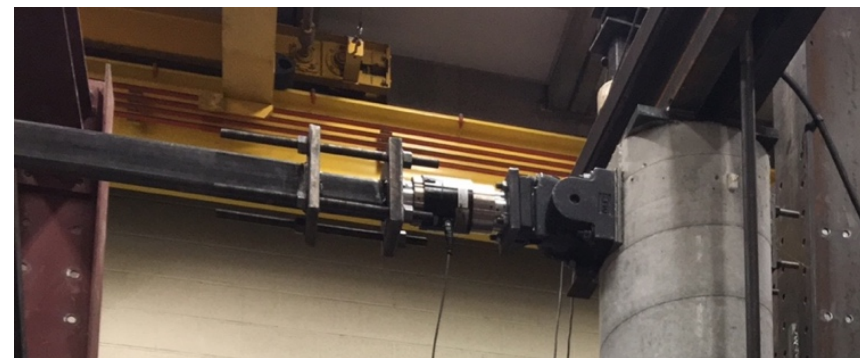

Figure 3.8 Pinned-end rigid link details

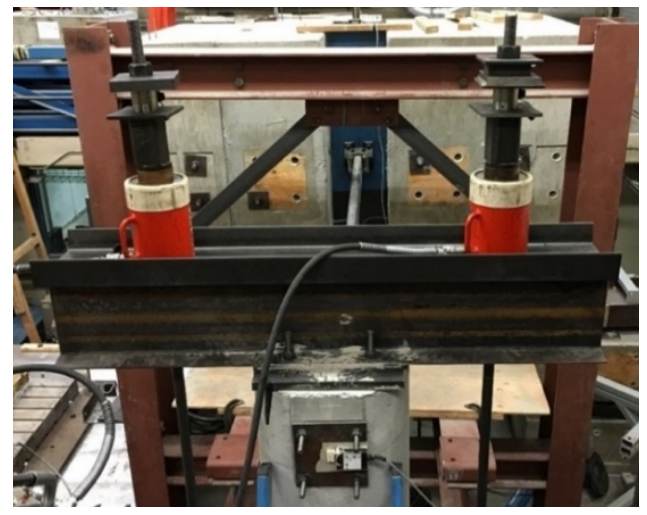

Figure 3.9 Axial load system

\subsubsection{Instrumentation}

All specimens were extensively instrumented to monitor global and local responses. Instrumentation comprised of strain gauges, linear variable displacement transducers (LVDTs), string potentiometers, load cells and accelerometers. Additionally, GO-Pro and high-definition video cameras were used to record damage propagation. Test setup details are illustrated in Figure 3.10.

The inertial forces transferred to the specimens were measured using a 50-kip MTS load cell integrated in the rigid link (Figure 3.8). The axial force was monitored using a 100-kip Honeywell load cell on each rod as shown in Figure 3.9. External absolute lateral displacements of the specimens were measured using two string potentiometers installed off the shake table on a rigid frame, one in the same direction of 
the loading to measure in-plane displacements and one perpendicular to the direction of loading to measure any out-of-plane displacement. Furthermore, the absolute displacement of the shake table was also measured using transducers incorporated into the shake table actuator. Slippage of the foundation block was also measured through the use of two LVDTs on one of its sides. Column relative in-plane displacement was calculated as the difference between in-plane string pot and the shake table transducer.

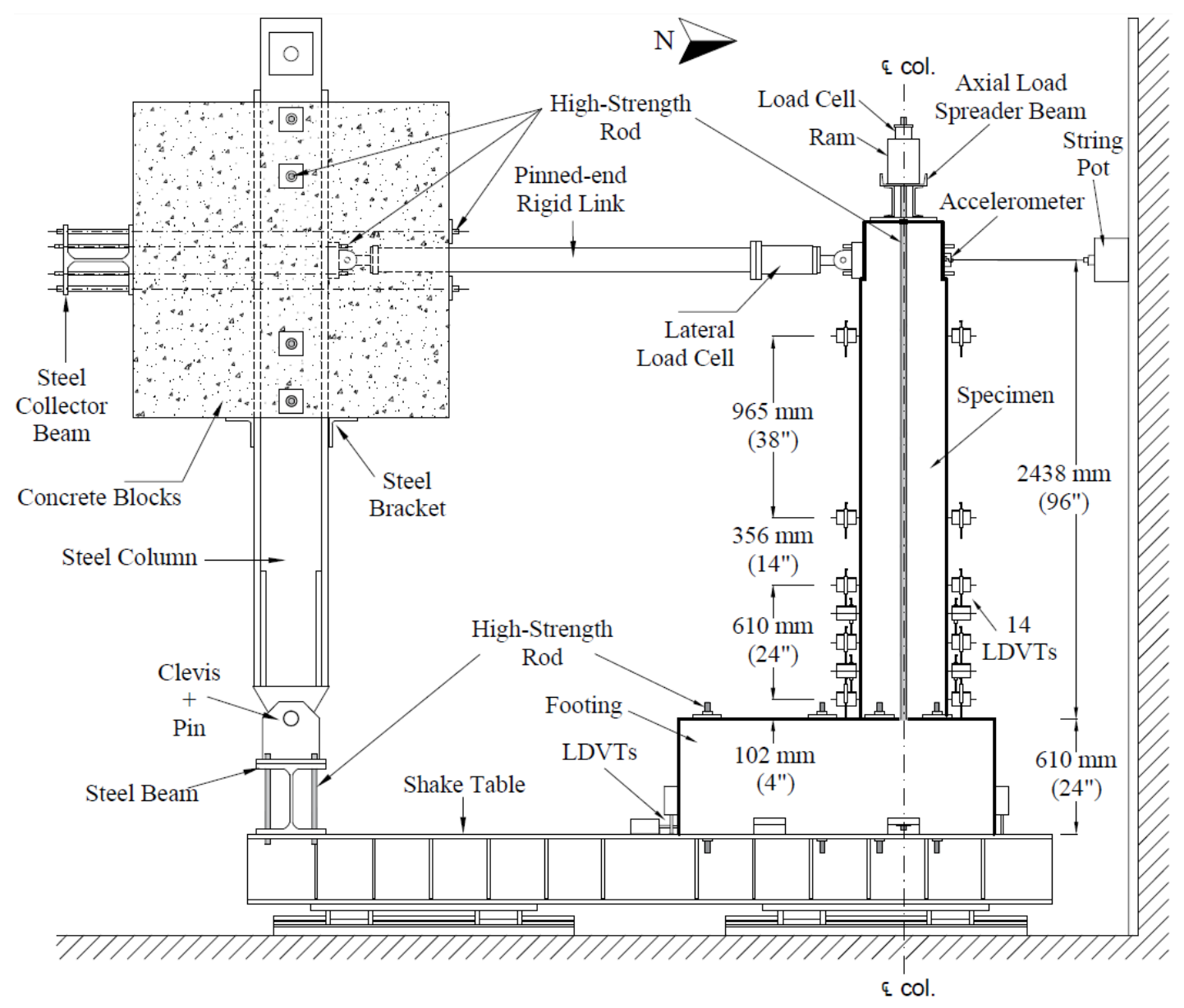

Figure 3.10 Test Setup and Instrumentation

Curvature and steel strains were measured. LVDTs were utilized in curvature estimation during the tests. Seven levels of LVDTs were instrumented in the North-South direction. The LVDTs were attached to a 5/16 in. diameter threaded rod that ran all the 
way through the column and were installed prior to the pouring. The location of curvature transducers is shown in Figure 3.10 and Figure 3.13(a). Strain gauges were placed on some rebars within the footing and above the footing at expected plastic hinge zone of columns to monitor strains on the longitudinal and transverse reinforcement. Strain gauges were installed on both the dowels and the vertical bars for specimens with lap splices at the expected plastic hinge zone of the columns, as depicted in Figure 3.11. A total of 17 strain gauges were installed in four sections of the rebars along the height of the columns with continuous reinforcement, whereas a total of 25 strain gauges were installed in the same four sections on the rebars along the height of the columns with lap splice at the hinge zone. Installation of strain gauges was done before the concrete pouring as shown in Figure 3.12. It is worth mentioning that concrete strains were not monitored by any device during testing, but from the longitudinal steel strain data could be possible to calculate these strains based on a linear strain profile for a circular column, as described later in this Chapter.

Two accelerometers were used to measure the accelerations for each specimen. One was installed on the column head and one on the footing (Figure 3.13(b)). An additional accelerometer was installed on top of the inertial mass system for comparison purposes. An internal accelerometer was used to measure the acceleration of the table.

Two Go-Pro cameras were used during the tests to capture damage of the specimens. One of them was installed on the south-east side of the footing to capture the damage in the plastic hinge zone (Figure 3.13(a)). The other camera was installed outside the shake table on the east side of the column to capture damage along the height of the 
specimens. All test data from LVDTs, load cells and strain gauges were collected using a National Instrument Data Acquisition System (DAQ) connected to a terminal computer. Data were displayed and recorded using the software LabView from National Instruments.

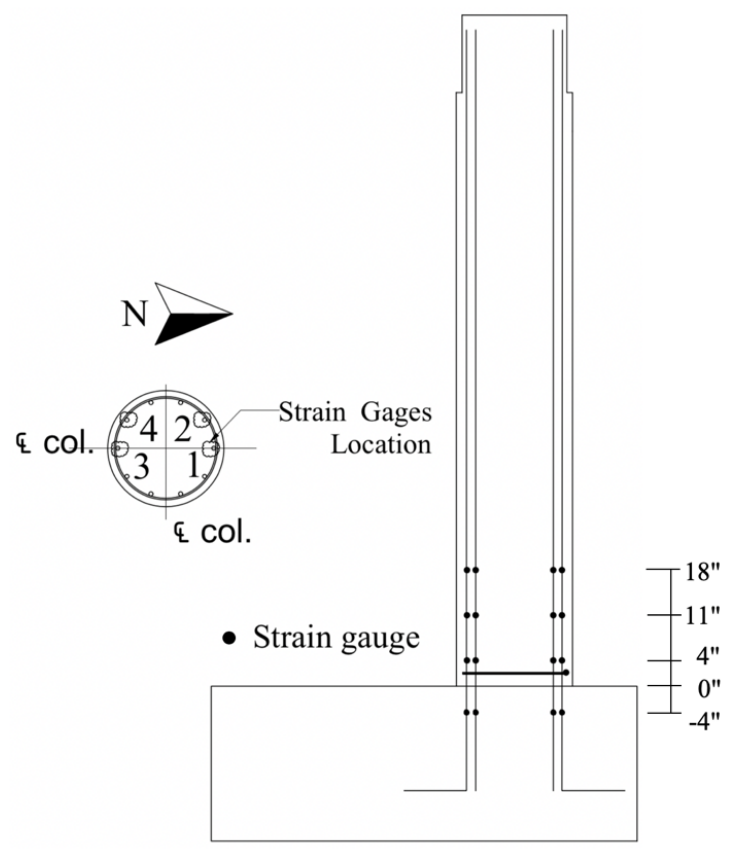

Figure 3.11 Distribution of strain gauges

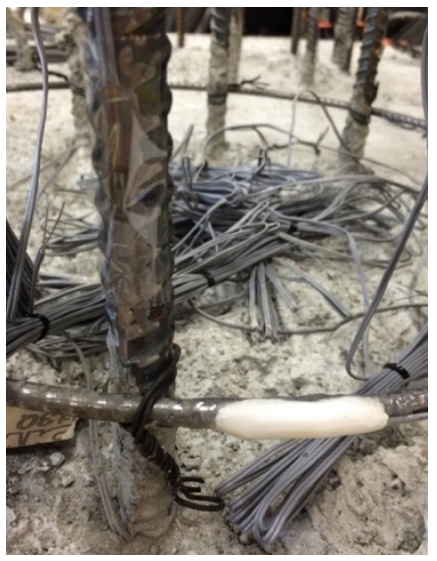

a)

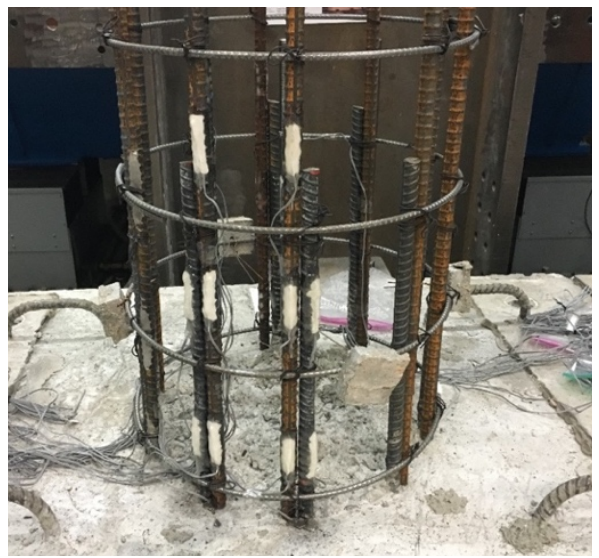

b)

Figure 3.12 Installation of strain gauges. a) Strain gauges on longitudinal reinforcement and hoop, b) Strain gauges on longitudinal reinforcement and dowel 


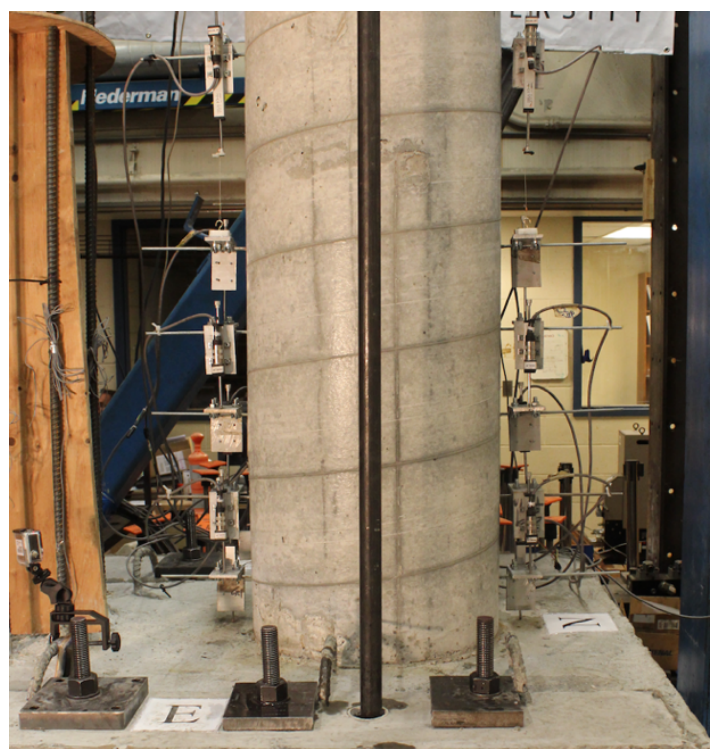

a)

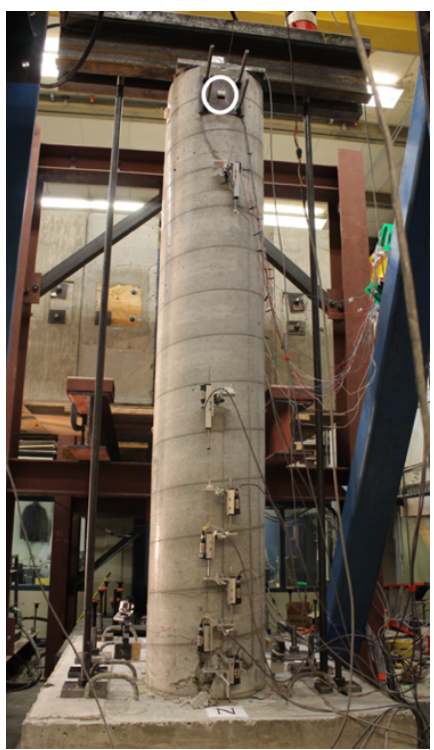

b)

Figure 3.13 a) LVDTs layout and one go-pro, b) Accelerometer on column head

\subsubsection{Ground Motion Selection}

Earthquake records from subduction and crustal earthquakes were considered. The purpose of using these sources of ground motions was to contrast the damage expected from a potential Cascadia subduction zone earthquake on substandard RC bridge columns. The crustal ground motion representing a typical duration motion was chosen to be the Capitola record recorded during the 1989 Loma Prieta earthquake with $M_{w}=6.9$, where $M_{w}$ is the moment magnitude. The subduction ground motion representing longer duration motions were chosen to be the Curico record of the 2010 Maule earthquake with $M_{w}=8.8$, and the Iwaki record of the 2011 Tohoku earthquake with $M_{w}=9.0$. These earthquake motions were selected from sets used in a numerical study of subduction zone strong motion records based on their number of inelastic cycles of pre-peak excursions for short period structures, i.e. $\mathrm{T}=0.5 \mathrm{sec}$ (Bazaez \& Dusicka, 2016). The short fundamental period was chosen as representative of conventional RC 
highway bridges. Among the numerous definitions of ground motion duration available in the literature (Bommer \& Martinez-Pereira, 1999), (Hancock \& Bommer, 2006) (Foschaar et al., 2012), the 5-95\% significant duration (Trifunac \& Brady, 1975) metric was calculated and is summarized for comparison in Table 3.2. This metric is calculated as the interval between the times at which $5 \%$ and $95 \%$ of the Arias Intensity of the ground motion have been recorded, representing $90 \%$ of the total accumulated energy. Instead of incrementally increasing the amplitude of the earthquake record, a significant amount of damage was intended on the first motion. Hence, each record needed to be scaled to achieve a similar target displacement ductility regardless of the duration of the record. The ductility level was calculated by relating the column top displacement to the yield displacement. The yield displacement was calculated as a combination of flexural and bond slip deformation, and was estimated as 0.76 in. The yield displacement due to flexural behavior was obtained from pre-test moment-curvature $(M-\phi)$ analysis performed in SAP2000 (2011), following Caltrans (2013) and AASHTO (2009) recommendations. The yield displacement due to slip of the tensile longitudinal reinforcement was calculated using the recommendation of Elwood \& Eberhard (2009), and where the uniform bond stress $u$ typically ranges from $6 \sqrt{f_{c}^{\prime}}$ to $12 \sqrt{f_{c}^{\prime}}$ psi (Sozen et al., 1992), (Lehman \& Moehle, 2000), (Sezen, 2002). In this part of the study, a uniform bond stress of $6 \sqrt{f_{c}^{\prime}}$ was used, based on the good results reported by Mehary et al. (2018) in modeling substandard bridge columns. 
Table 3.2 Ground motion records

\begin{tabular}{c|c|c|c|c|c|c}
\hline Earthquake & Station & $\begin{array}{c}\text { Magnitude } \\
\left(\mathrm{M}_{\mathrm{w}}\right)\end{array}$ & Component & $\begin{array}{c}\text { PGA } \\
(\mathrm{g})\end{array}$ & $\begin{array}{c}\text { Significant } \\
\text { Duration } \\
(\mathrm{sec})\end{array}$ & $\begin{array}{c}\text { N.I.C } \\
\text { pre-peaks } \\
\left(\mathrm{T}_{\mathrm{n}}=0.5 \mathrm{sec}\right)\end{array}$ \\
\hline $\begin{array}{c}\text { Loma Prieta } \\
(\text { Crustal })\end{array}$ & Capitola & 6.9 & $00(\mathrm{X})$ & 0.53 & 12 & 12 \\
\hline $\begin{array}{c}\text { Maule, Chile } \\
(\text { Subduction })\end{array}$ & Curico & 8.8 & $\mathrm{EW}(\mathrm{X})$ & 0.41 & 52 & 36 \\
\hline $\begin{array}{c}\text { Tohoku, } \\
\text { Japan } \\
(\text { Subduction })\end{array}$ & Iwaki & 9.0 & $\mathrm{NS}(\mathrm{Y})$ & 0.39 & 85 & 39 \\
\hline
\end{tabular}

The scale factors for each motion were obtained from nonlinear time-history analyses of a numerical model of the column using OpenSees (2013). The column was modeled using a distributed-plasticity approach and consisted of beam-column elements with six integration points between nodes. The Concrete 02 uniaxial material was used to model both confined and unconfined concrete. The longitudinal reinforcing steel was modeled using the Hysteretic uniaxial material in an effort to capture the pinching behavior observed in columns with lap splices as well as strength and stiffness degradation. Bond-slip was also considered by using a zero-length section (Zhao \& Sritharan, 2007). A simple illustration of the model is shown in Figure 2.14, and the OpenSees force-displacement relationships for the selected motions and chosen scale factors are shown in Figure 3.14. Since failure could not be predicted from the OpenSees model and based on the condition of the specimen after the first two amplitude motions, a third scale factor (amplitude C) was decided to be included in the test sequence. This factor was linearly interpolated from the previous two scale factors and had no specific 
target displacement ductility to be achieved. The scale factors considered for the experimental program are summarized in Table 3.3.

Table 3.3 Scale factors for the selected ground motions

\begin{tabular}{c|c|c|c}
\hline Ground Motion & Designation & $\begin{array}{c}\text { Scale } \\
\text { Factor }\end{array}$ & $\begin{array}{c}\text { Target Ductility } \\
(\mu)\end{array}$ \\
\hline \multirow{3}{*}{ Capitola X } & amplitude A & 1.00 & 4 \\
\cline { 2 - 4 } & amplitude B & 1.48 & 6 \\
\cline { 2 - 4 } & amplitude C & 1.75 & - \\
\hline \multirow{3}{*}{ Curico X } & amplitude A & 1.57 & 4 \\
\cline { 2 - 4 } & amplitude B & 1.68 & 6 \\
\cline { 2 - 4 } & amplitude C & 1.74 & - \\
\hline \multirow{3}{*}{ Iwaki Y } & amplitude A & 1.77 & 4 \\
\cline { 2 - 4 } & amplitude B & 1.92 & 6 \\
\cline { 2 - 4 } & amplitude C & 2.04 & - \\
\hline
\end{tabular}

As indicated in Section 3.3, a total of six specimens were constructed with nomenclature that represents the column number, type of detailing at column base and earthquake event considered; e.g. C2C-M referring to column 2 with continuous reinforcement and subduction ground motion, and C4S1-L referring to column 4 with splice 1 and crustal ground motion. The test order of the columns and description of the nomenclature used for each specimen is presented in Table 3.4. It is worth mentioning that based on the results of the first test series, the Curico record of the 2010 Maule subduction zone earthquake was chosen as the subduction motion input for the second test series. Figure 3.15 shows the original acceleration histories for the selected subduction and crustal motions. The 5\% damped elastic response spectra for amplitude A for each motion are shown in Figure 3.16, which are compared to the representative design response spectrum for a 1000-year event. 

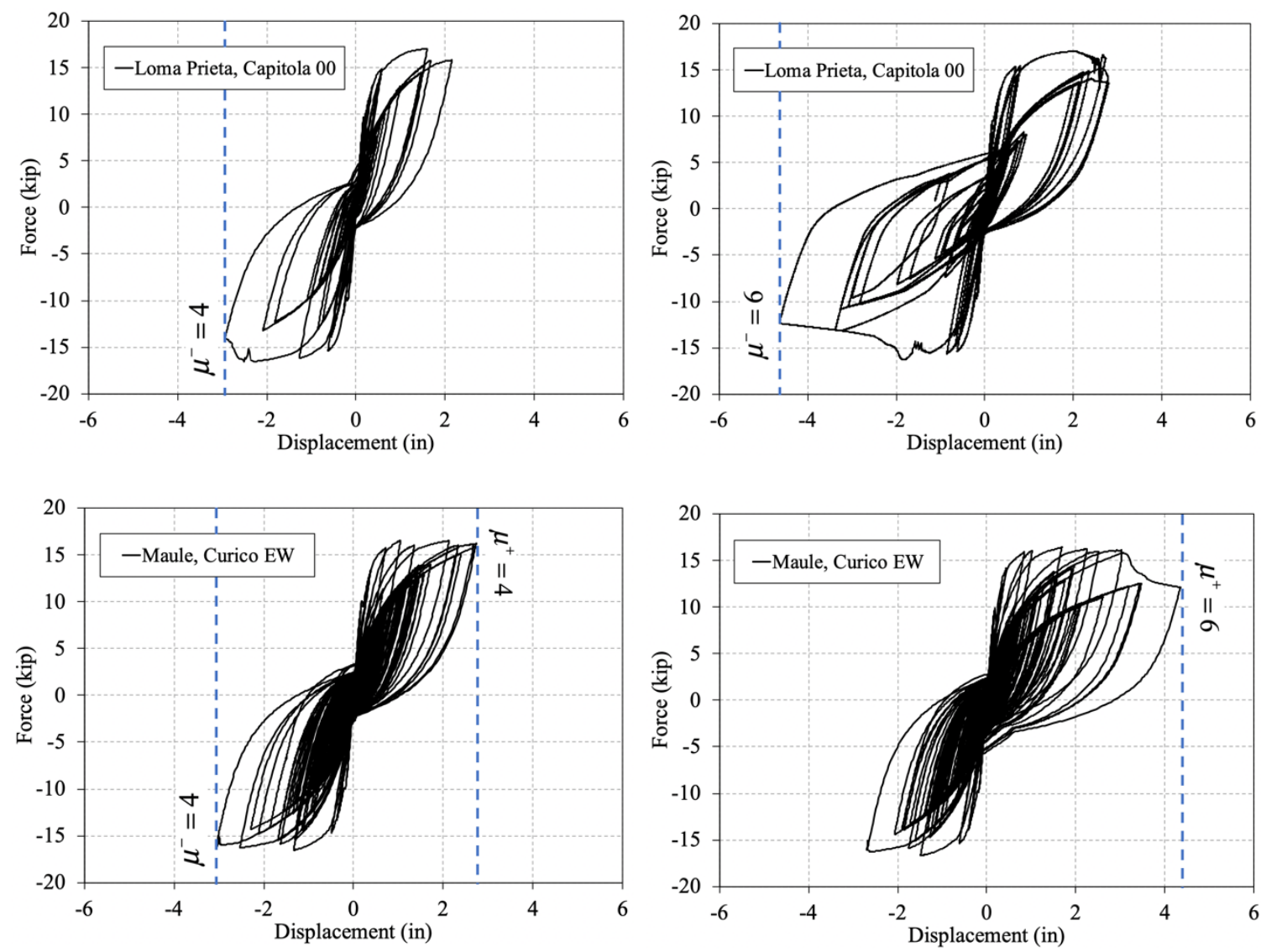

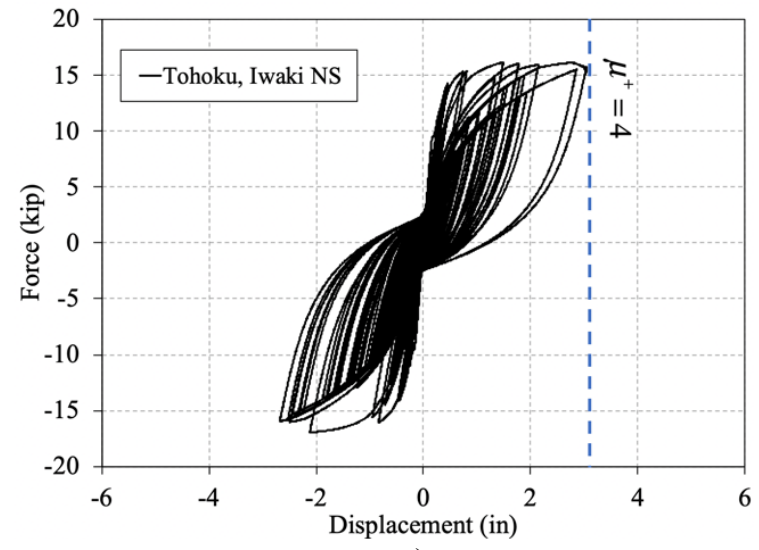

a)

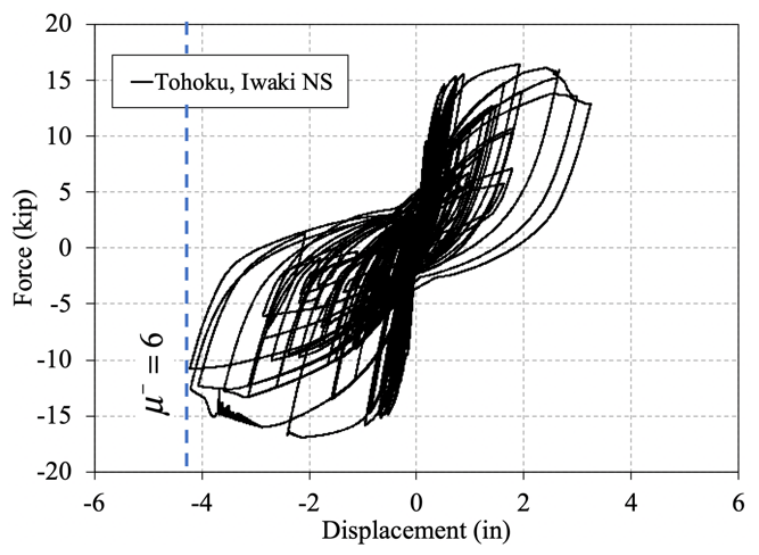

b)

Figure 3.14 Pre-test force displacement relationships for selected motions a) Amplitude A, b) Amplitude B 

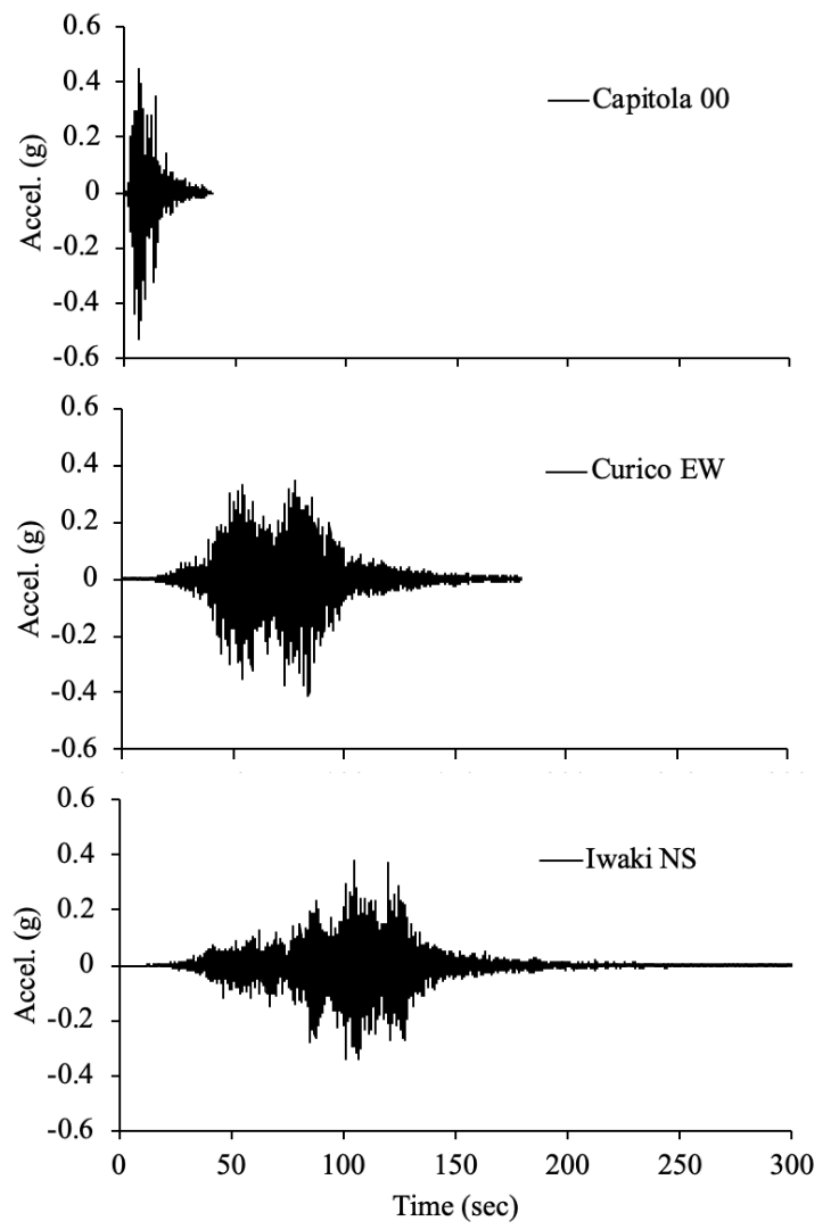

Figure 3.15 Original acceleration time histories

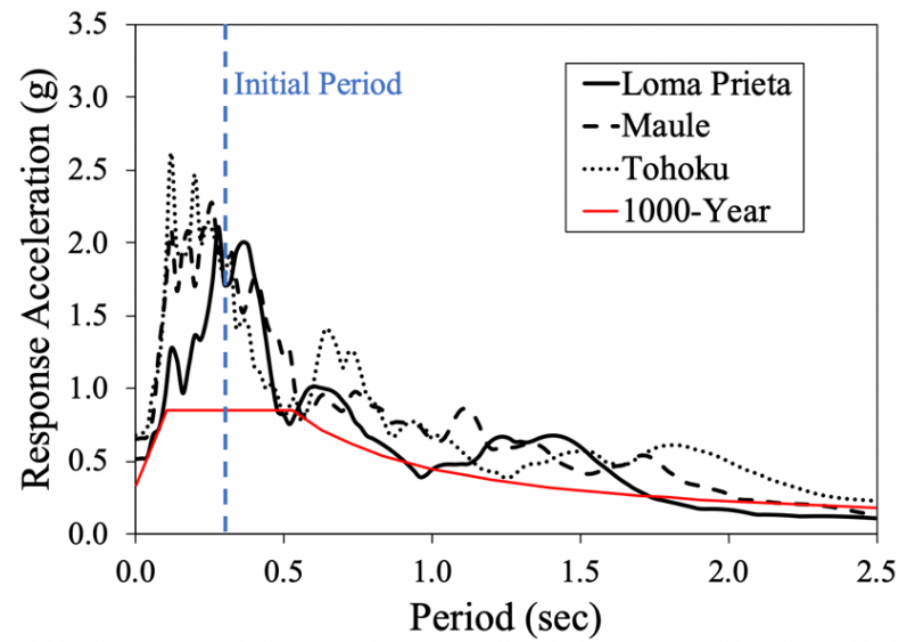

Figure 3.16 Elastic Response Spectra (5\% damping) for amplitude A 
Table 3.4 Column test order and nomenclature

\begin{tabular}{c|c|c}
\hline \multirow{2}{*}{ Test Series } & $\begin{array}{c}\text { Column } \\
\text { Nomenclature }\end{array}$ & Description \\
\hline \multirow{3}{*}{ I } & C1C-L & Column 1 - Continuous reinf. - Loma Prieta \\
\cline { 2 - 3 } & C2C-M & Column 2 - Continuous reinf. - Maule \\
\cline { 2 - 3 } & C3C-T & Column 3 - Continuous reinf. - Tohoku \\
\hline \multirow{3}{*}{ II } & C4S1-L & Column 4 - Splice 1 - Loma Prieta \\
\cline { 2 - 3 } & C5S1-M & Column 5 - Splice 1 - Maule \\
\cline { 2 - 3 } & C6S2-M & Column 6 - Splice 2 - Maule \\
\hline
\end{tabular}

The testing program started with a pulse test and a low-level white noise motion to determine initial properties of each specimen. Additionally, intermittent pulse tests and low-level white noise motions were conducted to measure the change in the natural period of the columns as a measure of damage progression. It is worth noting that the specimens were loaded in the north-south direction only.

\subsection{Experimental Results}

This section discusses the experimental results from the shake table testing of the two sets of substandard RC bridge columns. The first set considered specimens C1C-L, C2C-M, and C3C-T, which were tested using three different ground motion; one crustal and two subduction motions. The amplitudes used for each motion intended to achieve a similar target displacement ductility regardless of the duration of the record. Based on the results of the first test series, the Curico record of the 2010 Maule subduction zone earthquake was chosen as the subduction motion input for the second test series, which considered specimens C4S1-L, C5S1-M, and C6S2-M. Progression of testing and sequence of damage is demonstrated. It is worth noting that the sequence of damage was similar for all specimens but final state, and was as follow: concrete cracking, 
longitudinal reinforcements yielding, initial spalling of the concrete cover, complete spalling of the concrete cover, and longitudinal bar buckling or core crushing. The global behavior of the columns, in terms of column displacement, stiffness, and changes in period and damping is discussed. Local response in terms of strains in the reinforcement and section curvatures is also presented.

\subsubsection{Column C1C-L}

Column C1C-L was the first column tested. It was subjected to the Capitola X record from the 1989 Loma Prieta earthquake (crustal). Test results and discussion are presented in the following subsections.

\subsubsection{Progression of Observed Damage}

The specimen was first subjected to amplitude A of the selected ground motion. During this amplitude motion, the damage on the column was characterized by horizontal cracks developed all around the circumference of the column at the first levels of transverse reinforcement. Two major horizontal cracks at 8 in and 14 in above the column base were observed during testing, but were closed after shaking. After first bar yielding, initial spalling of concrete cover occurred at motion amplitude A. After the first motion amplitude, extend of spalling was up to 2 in from the interface, in the north and south faces of the specimen (Figure 3.17). Propagation of flexural cracks and extensive spalling of concrete at the hinge zone was observed during the motion amplitude $\mathrm{B}$. The spalled region reached an elevation of 8 in on the south side. Crushing of concrete at the column base on the north and south faces was observed. After shaking, one level of transverse reinforcement and the southernmost longitudinal bar were visible (Figure 
3.18(a)). Since visual inspection indicated that the specimen could undergo another event, motion amplitude $\mathrm{C}$ was then applied. The column reached its final damage state during this motion amplitude. When the specimen underwent the first large displacement cycles on each direction, many longitudinal bars buckled. The longitudinal bars buckled over an approximate length of 12 inches, which corresponds to 2 times the spacing of the transverse reinforcement. Two displacement cycles later, the column lost about $70 \%$ of its cross-sectional area due to loss on effectiveness of the second level of transverse reinforcement and crushing of concrete core (Figure 3.19). Thenceforth, the specimen started rocking about this section located at 8 inches from the column base. The ultimate mode of failure was crushing of concrete and longitudinal bar buckling at the bottom of the column in the plastic hinge zone. Lateral instability was followed and caused the column to deflect close to the inertial mass system maximum limit.

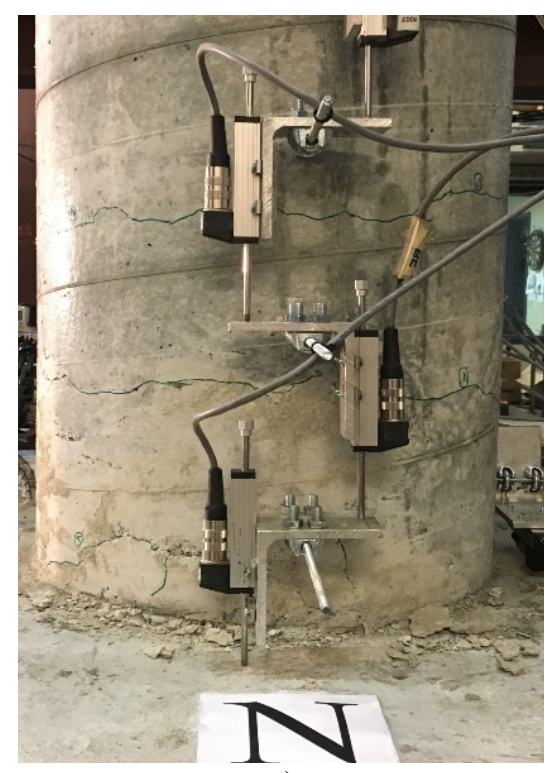

a)

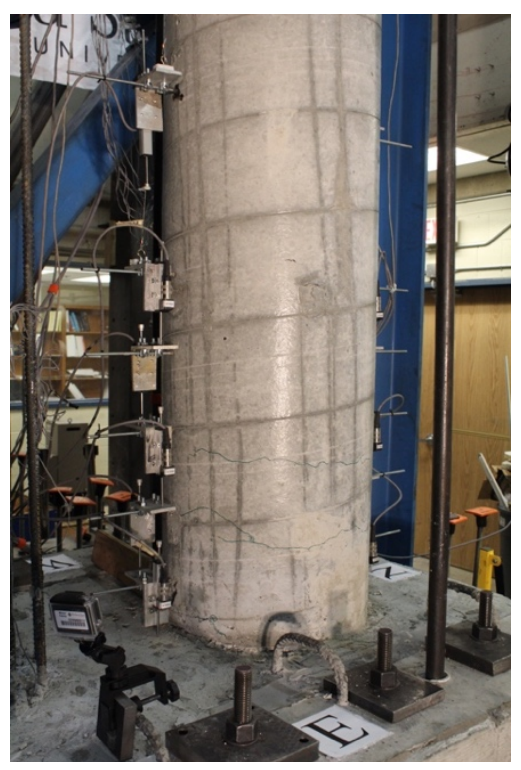

b)

Figure 3.17 Damage state of column C1C-L after applying motion amplitude A. a) North view, b) south-east view 


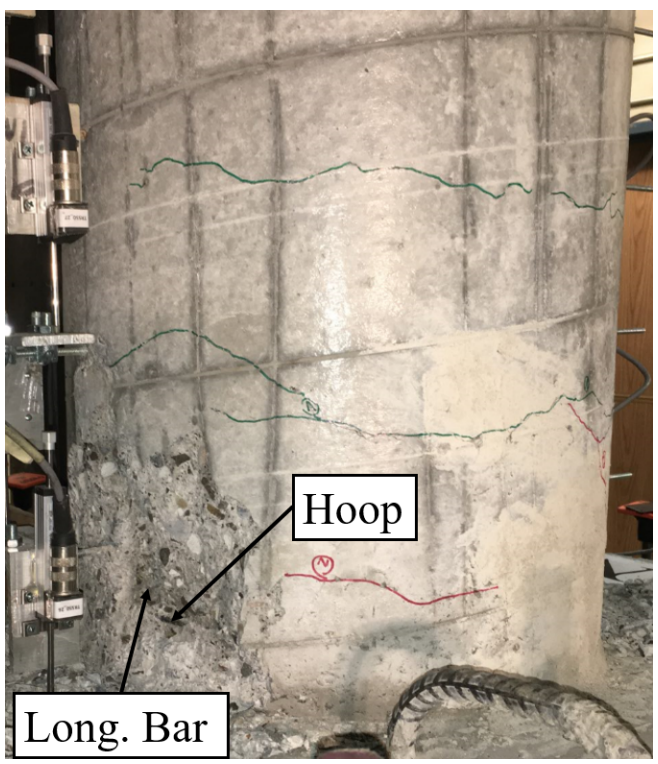

a)

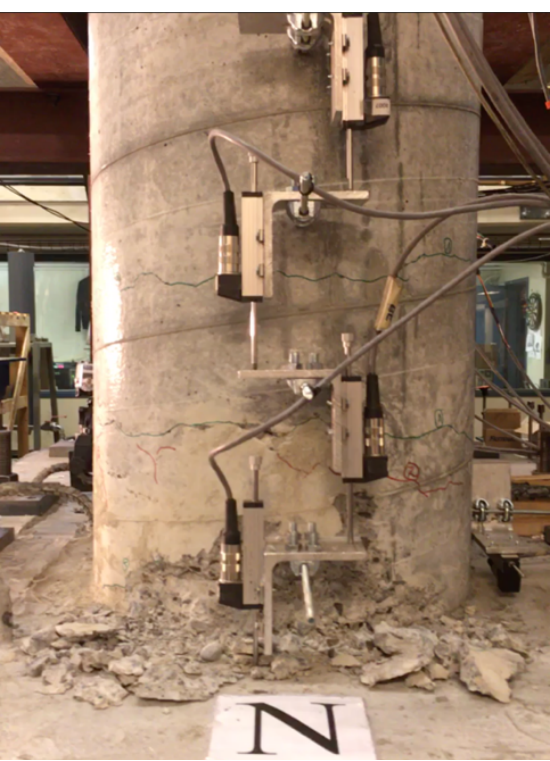

b)

Figure 3.18 Damage state of column C1C-L after applying motion amplitude B. a) North view, b) south-east view

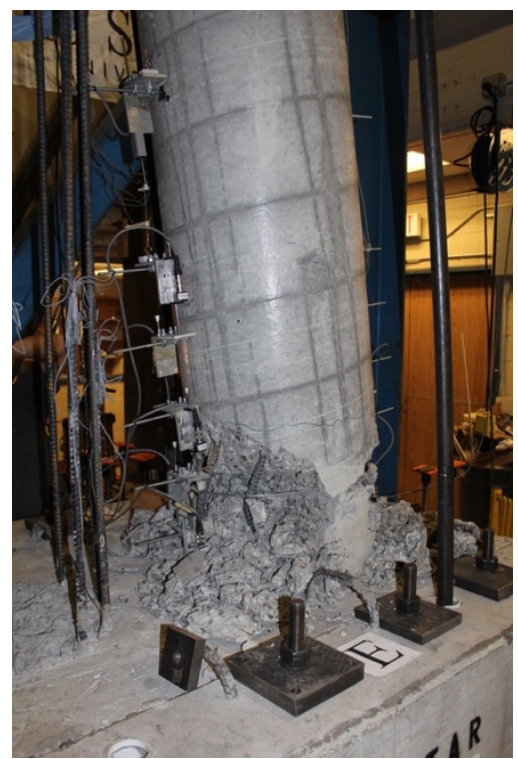

a)

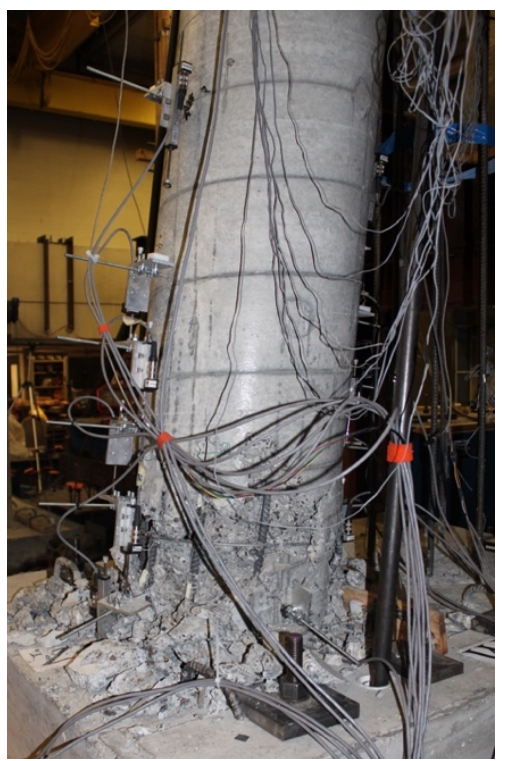

b)

Figure 3.19 Damage state of column C1C-L after applying motion amplitude C. a) South-east view, b) north-west view 


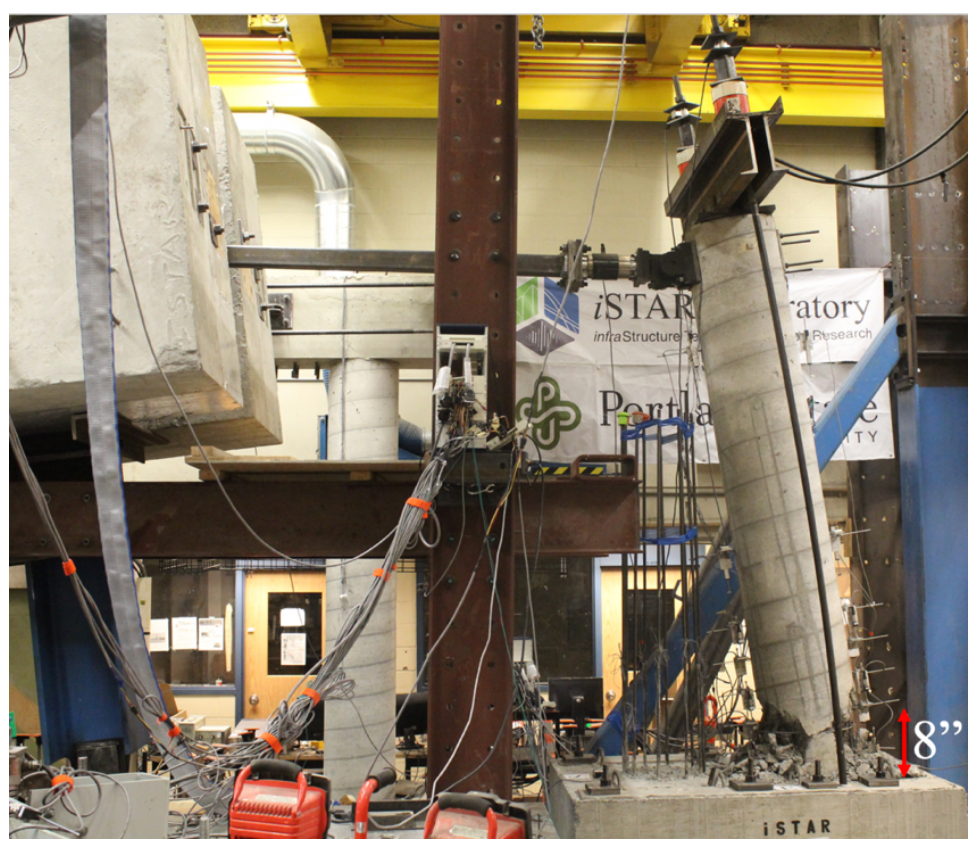

Figure 3.20 Final state of column C1C-L

As expected for a cantilever column, the damage was fully localized to the lower part of the column in the plastic hinge region. Figure 3.17 through Figure 3.19 show the different visual damage states for Column C1C-L after each ground motion amplitude. Figure 3.20 shows the specimen at completion of testing.

\subsubsection{Force-Displacement Relationship}

Force-displacement relationship is an important measure of structure performance. The measured hysteresis curves for column $\mathrm{C} 1 \mathrm{C}-\mathrm{L}$ for all ground motion amplitudes are shown in Figure 3.21 through Figure 3.23. Damage states are also identified on the force-displacement response histories. As was mentioned previously, the specimen was loaded in the north-south direction. This means that positive displacement direction corresponds to the north direction, and negative displacement direction is towards the south direction. 
The displacement on the hysteretic curves refers to the relative top column displacement between the column head and the top of the footing. It was calculated by subtracting the absolute displacement measured at the column head of the column from the shake table displacement. Lateral force (base shear) applied at the top of the column was calculated as the summation of inertia forces developed in the specimen. The lateral force coming from the inertial mass was measured by the load cell attached to the link connecting the inertia mass and the column. The specimen mass was also considered through the self-weight of the column (half of the column height), the mass of the swivel attached at the column top, and the mass of the axial load system. The portion of the specimen mass was then multiplied by the acceleration recorded at the head of the column at the same level of the pinned-end rigid link. Finally, the lateral force was corrected by considering the effects of the horizontal component of the axial load at large displacements.

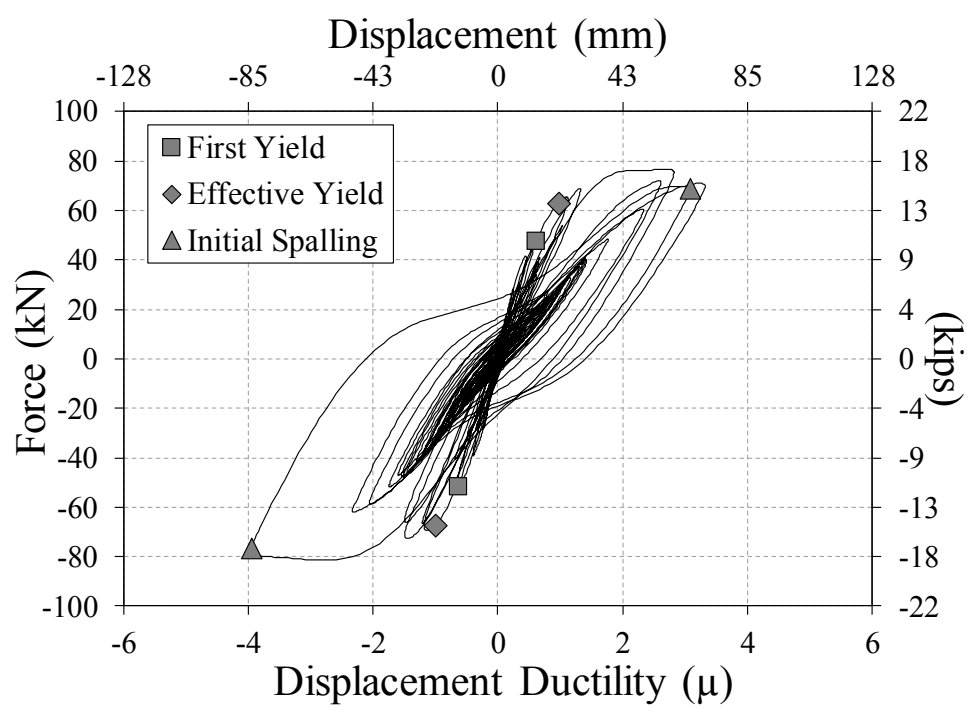

Figure 3.21 Hysteretic response for column C1C-L during motion amplitude A 


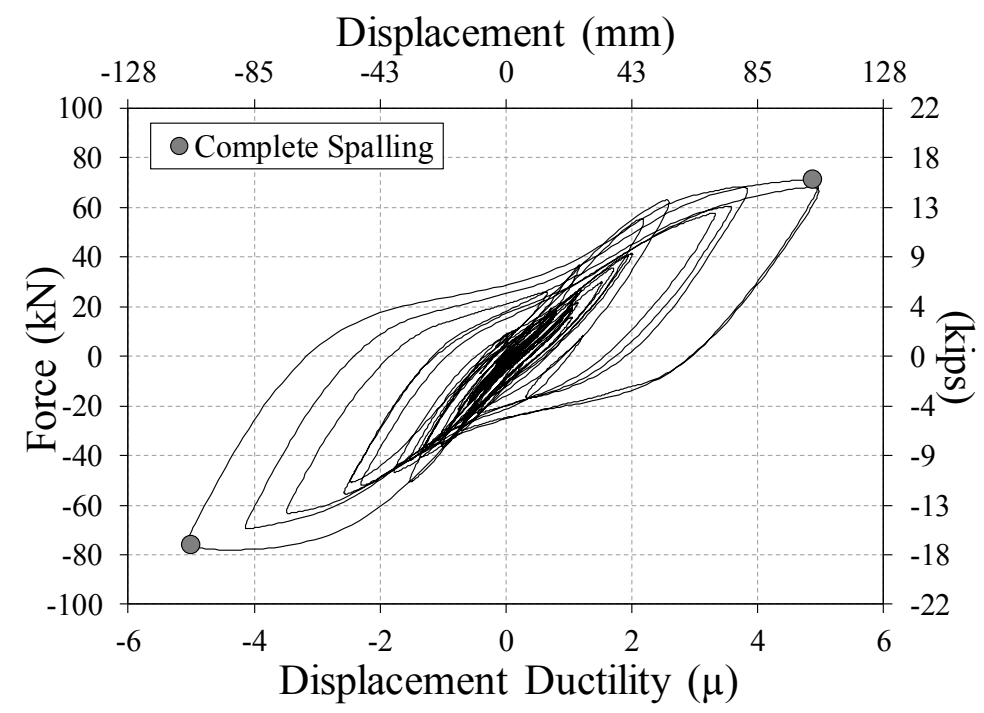

Figure 3.22 Hysteretic response for column C1C-L during motion amplitude B

Based on the experimental results, column C1C-L showed a hysteretic response typical of flexural behavior. For motion amplitude A, degradation of column stiffness was exhibited after first bar yielding. After this motion amplitude, the specimen did not exhibit a significant decrease in lateral strength (Figure 3.21). For motion amplitude B (Figure 3.22), the specimen was not able to reach the target displacement ductility of six due to the clear degradation in column stiffness; however, the column did not fail after motion amplitude B. Since visual inspection did not show extensive damage, it was decided to run motion amplitude $\mathrm{C}$. However, to prevent the risk of specimen collapse, a reduction in the applied axial load was made. Then, motion amplitude $\mathrm{C}$ was applied and caused the complete collapse of column C1C-L. It is worth mentioning that a significant loss in the load-carrying capacity was observed because of the buckling of many longitudinal bars. The failure of the column during this motion amplitude was evident by the large displacement shifting the hysteresis loops to the far left (Figure 3.23). Consequently, the part of the motion with extensive loss capacity was not considered 
(dashed-line). Despite the design deficiencies of column C1C-L, namely, low ratio of transverse reinforcement and no seismic detailing, this column exhibited a moderate ductile behavior and energy dissipation.

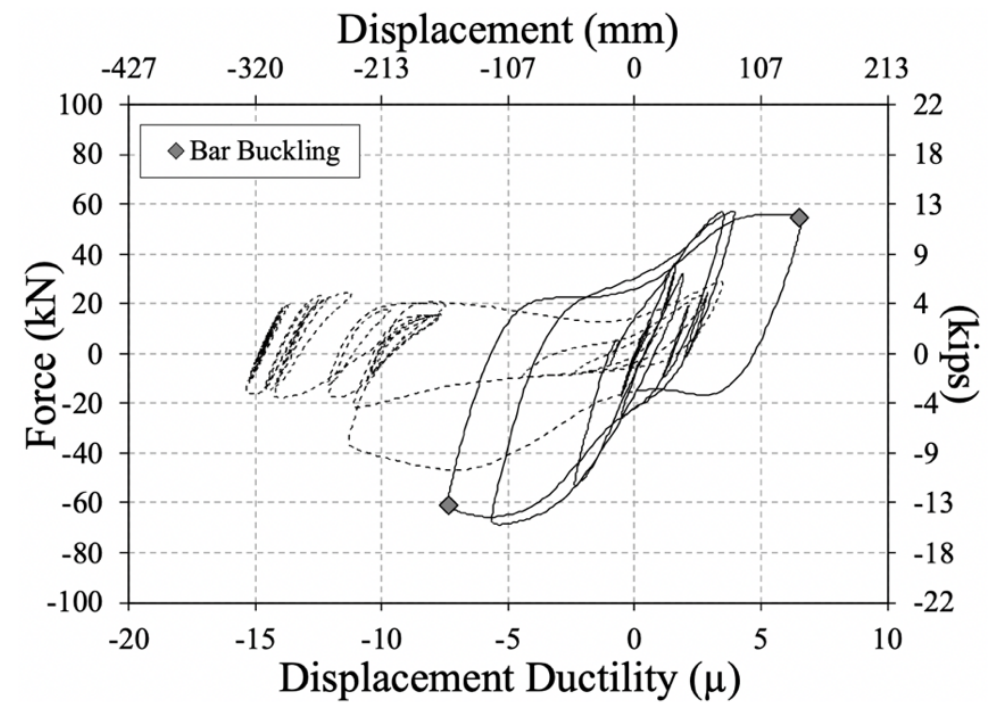

Figure 3.23 Hysteretic response for column C1C-L during motion amplitude C

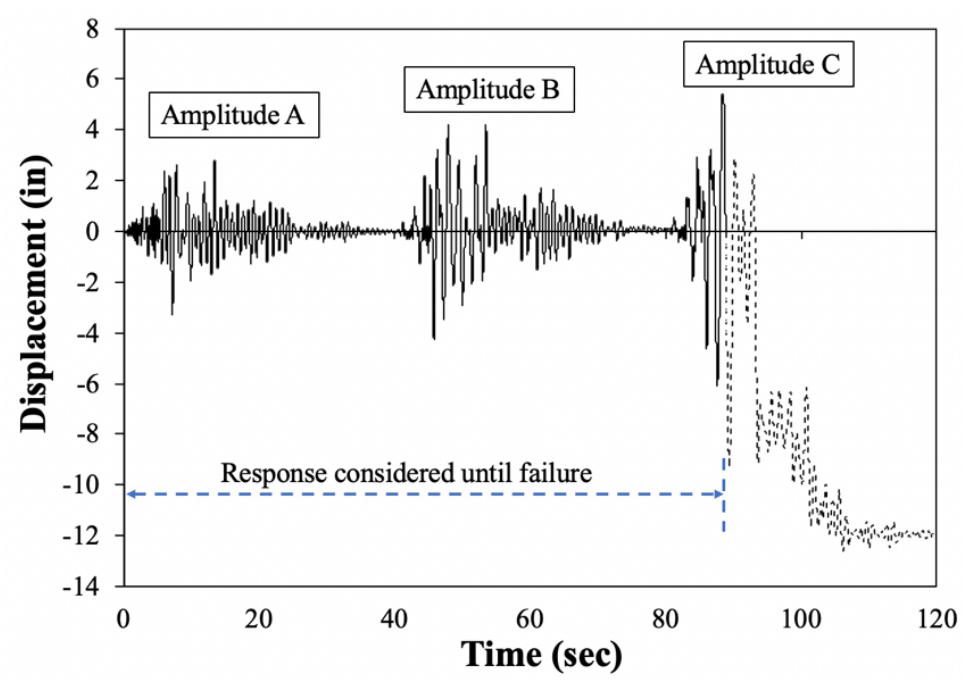

Figure 3.24 Column displacement history for column C1C-L 
Table 3.5 Summary of the measured performance for C1C-L

\begin{tabular}{c|c|c|c}
\hline Property & Amplitude A & Amplitude B & Amplitude C \\
\hline $\begin{array}{c}\text { Max. Displacement } \\
\text { in. (mm) }\end{array}$ & $3.3(84.2)$ & $4.2(107.7)$ & $6.1(155.0)$ \\
\hline $\begin{array}{c}\text { Max. Drift Ratio } \\
(\%)\end{array}$ & 3.45 & 4.45 & 6.35 \\
\hline $\begin{array}{c}\text { Max. Displacement } \\
\text { Ductility Level }(\mu)\end{array}$ & 3.9 & 5.0 & 7.4 \\
\hline $\begin{array}{c}\text { Residual } \\
\text { Displacement in. } \\
(\text { mm) }\end{array}$ & $0.02(0.5)$ & $0.06(1.5)$ & $12(304.8)$ \\
\hline $\begin{array}{c}\text { Max. Base Shear } \\
\text { kips }(\mathrm{kN})\end{array}$ & $18.3(81.5)$ & $17.5(78.0)$ & $15.4(68.7)$ \\
\hline
\end{tabular}

The maximum displacement for each earthquake motion amplitude, and its corresponding drift ratio and ductility level are presented Table 3.5. Reported displacement ductility levels are based on the effective yield displacement, which was found to be $0.84 \mathrm{in}$. This value was computed using the reduce stiffness method assuming an elasto-plastic idealization, where the secant stiffness passes through first yielding of the longitudinal steel while the plastic portion had zero slope, and passes through the peak lateral strength. These values are the peak displacement values an do not correspond to the peak lateral force values, which are also listed in Table 3.5. The displacement history is shown in Figure 3.24. It can be seen that once bar buckling occurred and the load carrying capacity dropped due to crushing of the concrete core, the specimen swung in the south direction in a whip like fashion, which generated large displacement in the negative displacement direction. Based on this fact, only a part of the response was considered, and it is represented by the solid line in Figure 3.24. Therefore, maximum displacement for this motion amplitude was considered 6.1 in not 9.3 in, where the 
capacity at 9.3 in dropped by about $55 \%$. It is seen in Figure 3.24 and in Table 3.5 that the column C1C-L experienced little residual displacement for the first two motion amplitudes. There was a residual displacement of 12 in during motion amplitude $\mathrm{C}$ due to collapse of the specimen.

\subsubsection{Dynamic Properties}

Low-level white noise signals, as well as pulse tests, were performed before and in between earthquake motion amplitudes to monitor the change in the dynamic properties due to damage progression in the specimen. Natural periods were determined from Fourier analysis of the accelerations measured at the top of the column. The damping ratio was calculated from the free vibration portion of the accelerograms recorded at the top of the specimen using the decrement logarithmic method (Chopra, 2001). Since the free-vibration portion of the accelerogram does not have a perfect exponential decay, the accelerogram was divided into various intervals for which a damping ratio was calculated. Then, a mean value was calculated for the damping ratio. A summary of the computed periods and mean damping ratio values and standard deviations are presented in Table 3.6. From the results, it is clear that the computed natural periods were lengthened with the increased level of excitation, what confirmed the deterioration of column stiffness. It is worth mentioning that the natural period at final damage state could not be computed due to the collapse of the specimen. Moreover, the elongated period after motion amplitude B is almost twice the initial cracked period of the column. The variation in damping followed the same trend, i.e., it increased with 
the progression of the damage from $1.9 \%$ to $4.4 \%$. In general, good agreement was observed between the results of both tests.

Table 3.6 Measured dynamic properties for C1C-L

\begin{tabular}{c|c|c|c|c}
\hline \multirow{2}{*}{ Sequence } & \multicolumn{2}{|c|}{ Period, $T_{n}(\mathrm{sec})$} & \multicolumn{2}{c}{ Damping ratio, $\zeta(\%)$} \\
\cline { 2 - 5 } & Pulse test & W-N random test & $\mu_{X}$ & $\sigma_{X}$ \\
\hline Initial & 0.35 & 0.36 & 1.9 & 0.27 \\
\hline amplitude A & 0.59 & 0.60 & 4.1 & 0.77 \\
\hline amplitude B & 0.62 & 0.67 & 4.4 & 0.38 \\
\hline
\end{tabular}

\subsubsection{Strains}

Reinforcing steel strains were recorded at numerous locations throughout the specimen using strain gages. The strain gauges were placed at different levels on four longitudinal bars on the north and south sides. The location of strain gauges was discussed in Section 3.4.1. (See Figure 3.11). The designation used in the figures is as follows: column position ( $\mathrm{N}$ : north or $\mathrm{S}$ : south), the bar number (1, 2, 3 or 4$)$, and the location (level) along the height (F: footing, L1: level 1, L2: level 2, and L3: level 3). For example, N-2-L2 corresponds to North - bar \#2 - Level 2. Figure 3.25 through Figure 3.28 show some of the recorded steel strain histories during earthquake motion amplitudes A and B for the tested specimen. The yield strain for the longitudinal bars was calculated as the ratio between the yielding stress reported in Table 3.1 and the modulus of elasticity of the steel $(29,000 \mathrm{ksi})$. Average yielding strain of $0.0018 \mathrm{in} / \mathrm{in}$ was then calculated for the longitudinal (No.5 bars) reinforcement. Based on the measured values, peak steel strain profiles were derived and plotted (Figure 3.29) for Specimen C1C-L along the plastic hinge. In these figures, some strain measurements are missing due to either damage during concrete pouring or failure during testing. A maximum strain of 
$0.017 \mathrm{in} /$ in was measured in the south rebar during motion amplitude $\mathrm{A}$, which corresponds to 10 times the yield strain $\left(\varepsilon_{y}\right)$. In general, steel strains were slightly larger in the south side of the column.

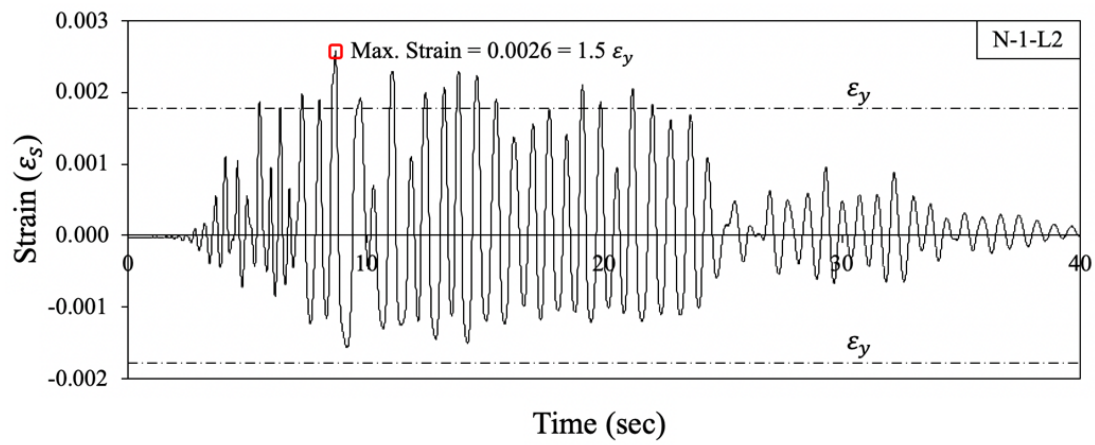

Figure 3.25 Steel strain history for strain gauge N-1-L2 for column C1C-L during motion amplitude $\mathrm{A}$

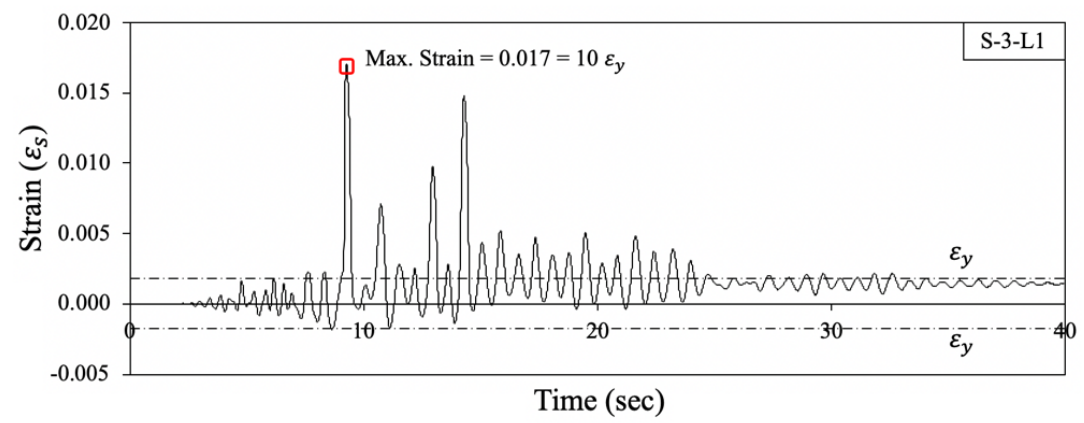

Figure 3.26 Steel strain history for strain gauge S-3-L1 for column C1C-L during motion amplitude A

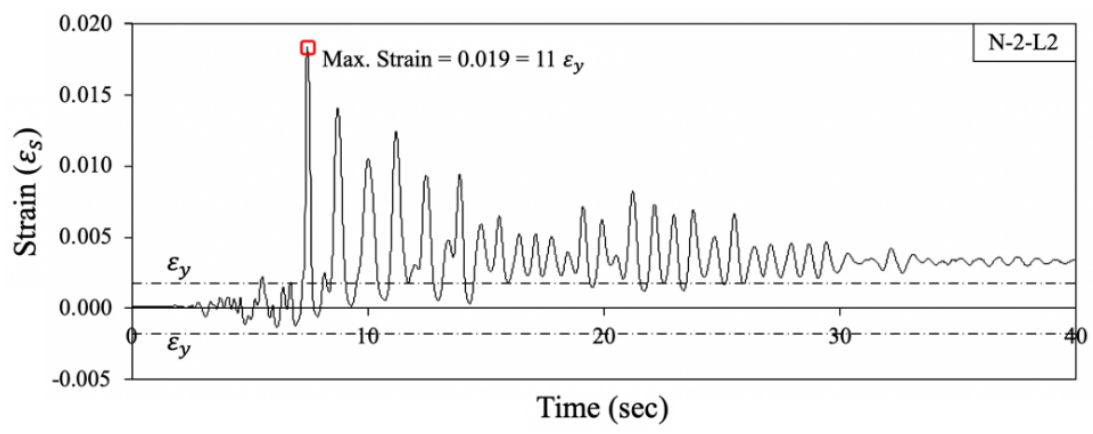

Figure 3.27 Steel strain history for strain gauge N-3-L2 for column C1C-L during motion amplitude B 


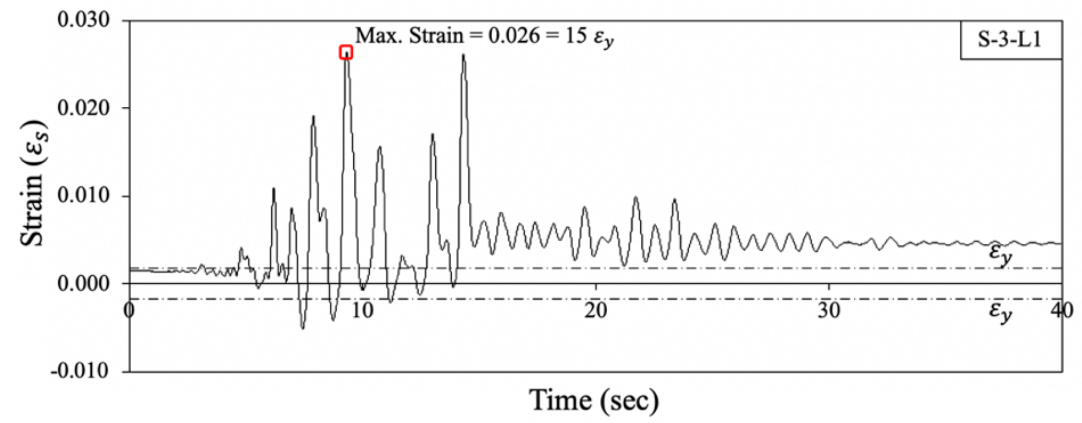

Figure 3.28 Steel strain history for strain gauge S-3-L1 for column C1C-L during motion amplitude B

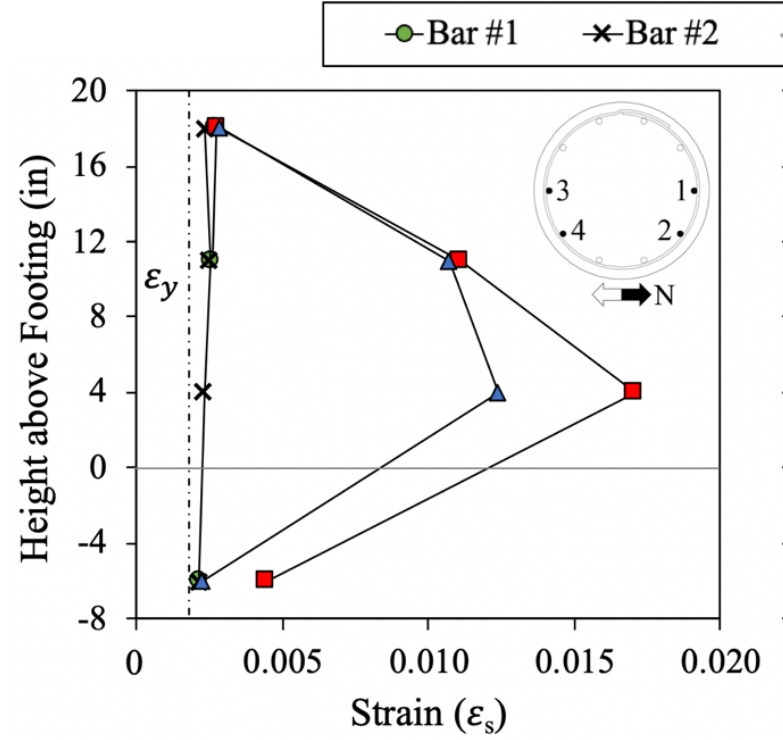

a)
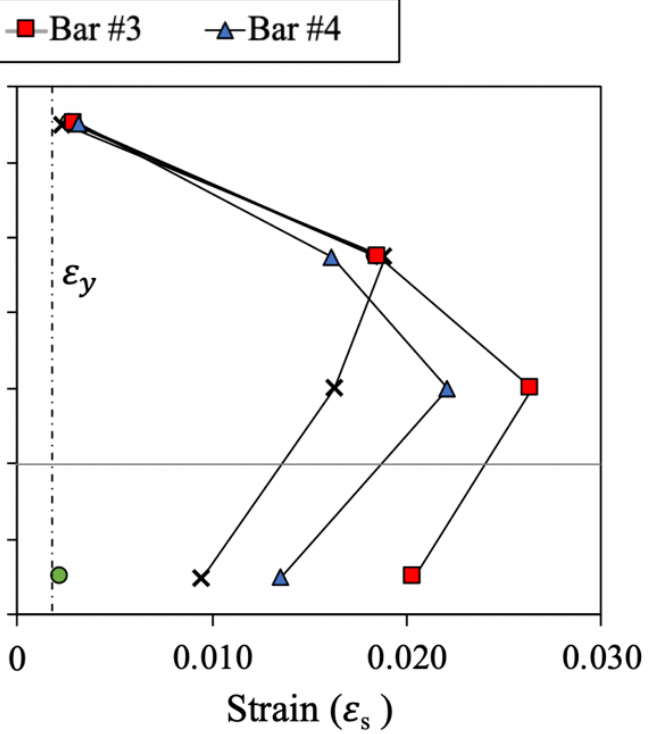

b)

Figure 3.29 Peak steel strain profile for column C1C-L. a) Amplitude A, b) Amplitude B

\subsubsection{Curvature}

Curvatures are an important measure for flexural-dominated RC columns where they increase significantly in the column plastic hinge region. Linear variable displacement transducers (LVDTs) were used to measure the average curvature along the height of the column. The average curvature was calculated by dividing the difference between the recorded deflections of the two opposite transducers by the gauge length and by the total horizontal distance between the instruments. The calculation is based on 
Bernoulli's principle of plane sections. The curvature instrumentation details were presented in 3.4.1.

The peak curvature profile along the height is also important to investigate the column behavior and is shown in Figure 3.30 for each motion amplitude. The points in the curvature profiles represent the curvature measured at the specific location and in correspondence to the maximum and minimum peak values of column displacement. The curvature profiles in each direction were fairly symmetric, except for motion amplitude $\mathrm{C}$, where the large negative curvatures are due the large loss in column capacity. This result agrees with the final damage state of column C1C-L, where the column tilted towards the south due to the collapse of the specimen. Also, as it was expected for columns tested on single curvature, large curvature values were measured at the base of the column, where the moments were larger and induced substantial nonlinear deformation. It is shown in the figure that the desired behavior was achieved in the plastic hinge region where the visible damage occurred (concrete cracks and spalling).

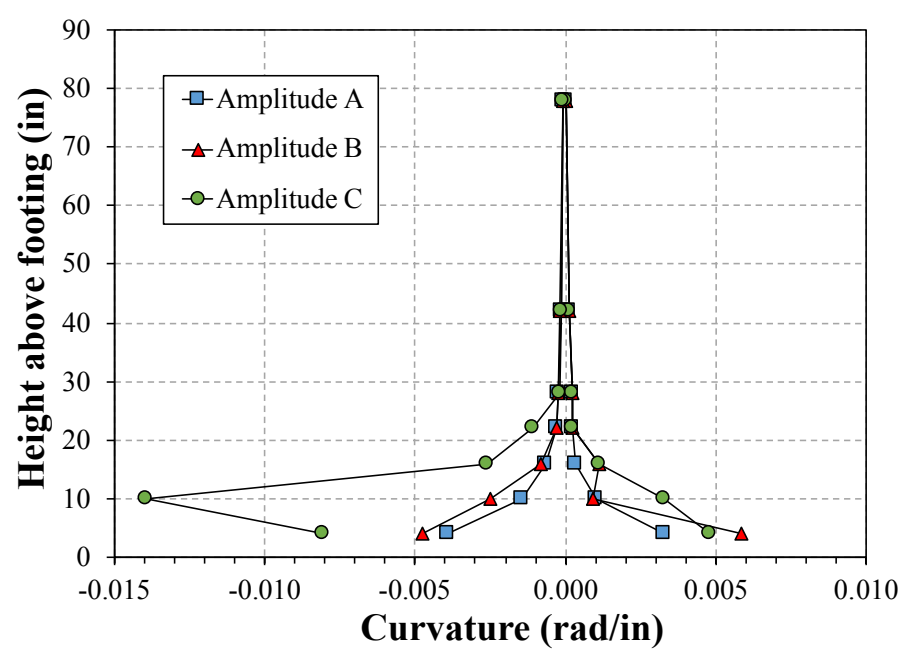

Figure 3.30 Average curvature profiles in column C1C-L 


\subsubsection{Axial Forces}

The axial force history for specimen C1C-L is shown in Figure 3.31. The target force was 75 kips ( $334 \mathrm{kN}$ ) for motion amplitude $\mathrm{A}$ and $\mathrm{B}$. For motion amplitude $\mathrm{C}$, the applied axial load was reduced to 60 kips $(267 \mathrm{kN})$ to prevent any risk that might have been occurred in case of collapse. The axial force plotted do not include the weight of the spreader beam and column dead load. Although the experiments were designed to keep the same level of axial force during the test sequence, fluctuations were observed in during testing in each earthquake motion. The maximum deviation from the target axial force was $-3.3 \%$ and $+24.3 \%$ in motion amplitude $\mathrm{A},-2.8 \%$ and $+28.6 \%$ in motion amplitude $\mathrm{B}$, and $-2.2 \%$ and $+38.5 \%$ in motion amplitude $\mathrm{C}$. The variations of the axial force may be attributed to the change in axial loads in the treaded rods as the specimen displaced. Normally, the accumulators compensate for this, but the hinging action of the load transfer beam created a less stiff axial load system. The less stiff system caused the hydraulic ram to displace further and faster than its ability to maintain constant axial load.

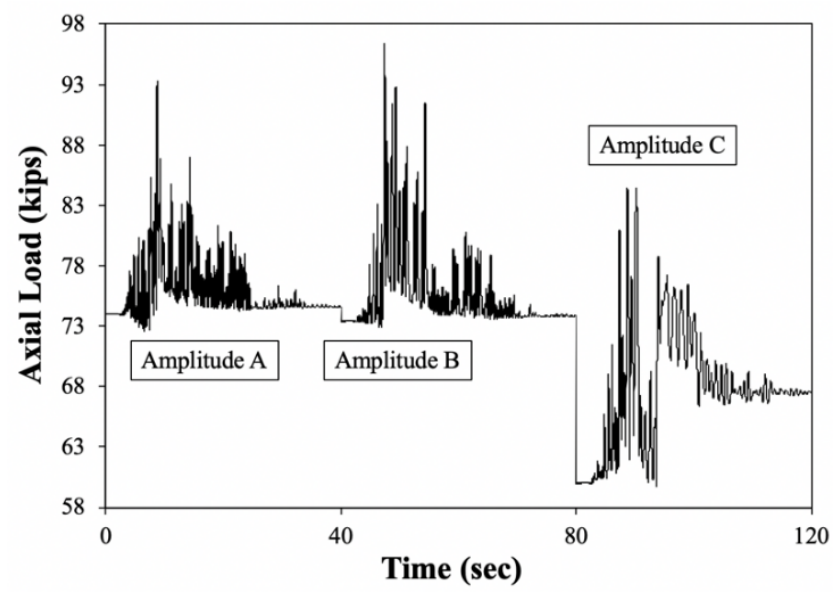

Figure 3.31 Axial force history for column C1C-L 


\subsubsection{Shake Table Output Motions}

Replicating exact ground motions can be challenging. Tuning the shake table before testing can improve the correlation between target and achieved shake table motions. The performance of shake table systems is defined as the ability of these systems to accurately reproduce the input signals. However, there are inevitably some differences. For the input motion applied column C1C-L, small differences between the target and achieved accelerations reproduced by the shake table were observed despite the fine-tuning applied to the system. To determine the accuracy of the reproduced signals, the time history accelerations recorded from the shake table were compared with the input acceleration at each motion amplitude (Figure 3.32(a)). Table 3.7 shows the target and achieved values of peak table acceleration (PGA), velocity (PGV), and displacement (PGD) for each motion amplitude. In addition to the peak responses comparison, $5 \%$ damped elastic acceleration response spectrums were calculated from the target and achieved acceleration signals for each motion amplitude, as depicted in Figure 3.32(b). Also, it is shown in the same figures the measured initial and final period of the specimen. Although there are variations in the target and achieved elastic response accelerations for all the motions, these discrepancies are more important at the low period portion of the spectrum. The impact of these discrepancies is not significant since the potential specimen's response is at higher periods as it is shown in the spectrums. Therefore, the table performance is judged as satisfactory for the period range of interest. 


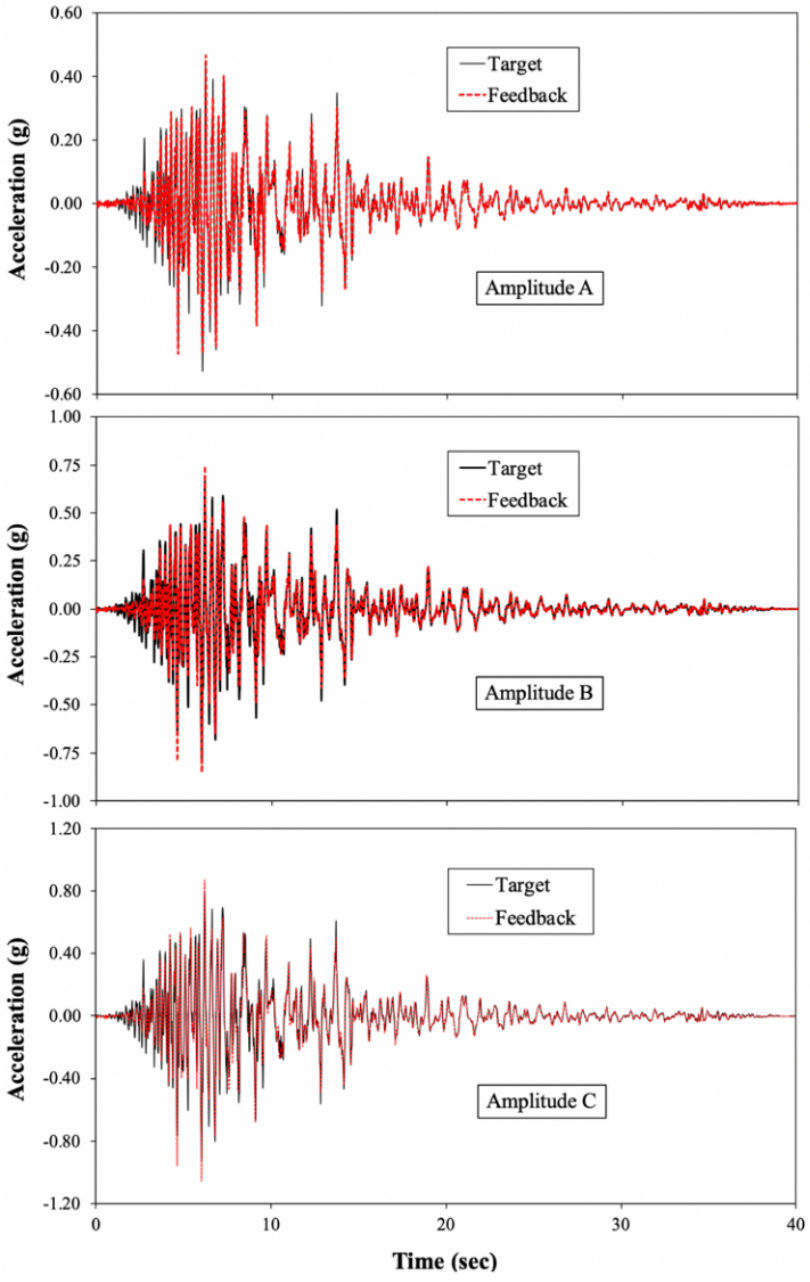

a)
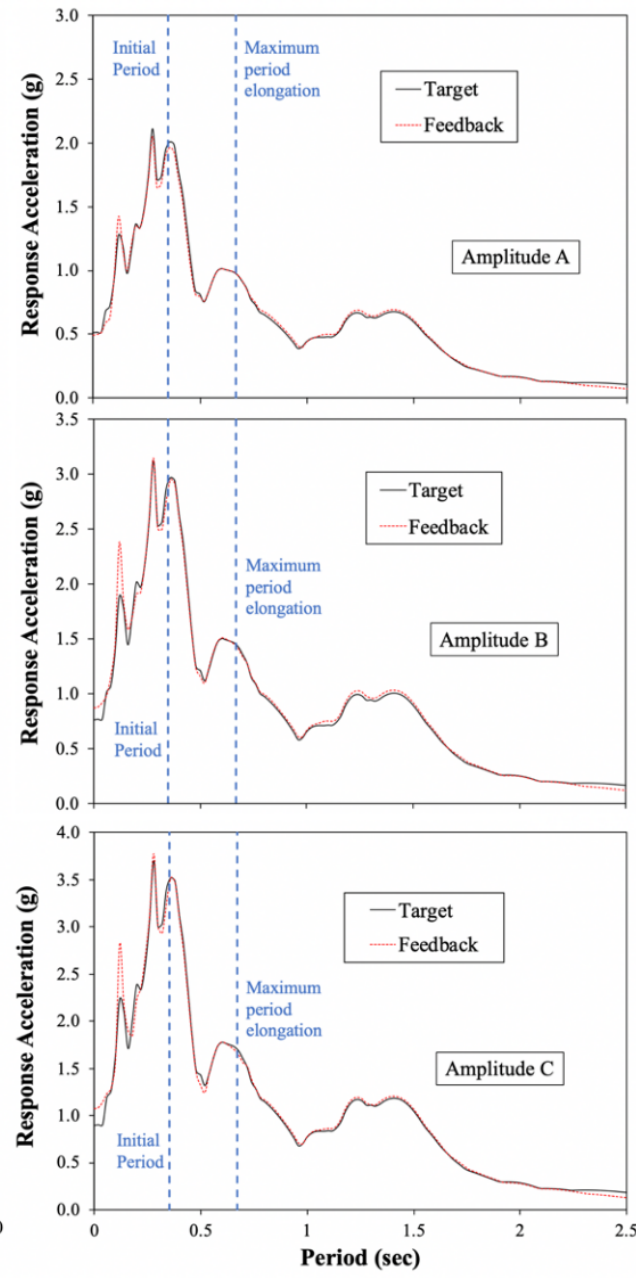

b)

Figure 3.32 Shake table performance for Capitola X. a) Acceleration time histories, b) 5\% damped elastic response spectra

Table 3.7 Target and achieved peak table responses for Capitola $\mathrm{X}$

\begin{tabular}{c|c|c|c|c|c|c}
\hline \multirow{2}{*}{ Amplitude } & \multicolumn{2}{|c|}{ PGA (g) } & \multicolumn{2}{c|}{ PGV (in/sec) } & \multicolumn{2}{c}{ PGD (in) } \\
\cline { 2 - 7 } & Target & Achieved & Target & Achieved & Target & Achieved \\
\hline A & 0.53 & 0.51 & 14.75 & 14.69 & 2.97 & 2.64 \\
\hline B & 0.78 & 0.85 & 21.83 & 22.26 & 4.39 & 3.92 \\
\hline C & 0.92 & 1.06 & 25.82 & 24.75 & 5.19 & 4.66 \\
\hline
\end{tabular}




\subsubsection{Column C2C-M}

Column C2C-M was the second column tested. It was subjected to the Curico X record from the 2010 Maule earthquake (subduction). Test results and discussion are presented in the following subsections.

\subsubsection{Progression of Observed Damage}

The specimen was first subjected to amplitude A of the selected ground motion. During this amplitude motion, the damage on the column was characterized by horizontal cracks developed all around the circumference of the column at the first levels of transverse reinforcement. Three major horizontal cracks at 8 in, 14.6 in, and 20 in above the column base were observed during testing but were closed after shaking. The specimen also exhibited minimum vertical cracking in the plastic hinge region. Following first bar yielding, initial spalling of concrete cover occurred at motion amplitude A. After the first motion amplitude, the extent of spalling of column $\mathrm{C} 2 \mathrm{C}-\mathrm{M}$ was considerably more than column C1C-L for the same amplitude motion. The spalled length was around 8.7 in and 6 in from the interface, in the north and south faces of the specimen, respectively (Figure 3.33). Propagation of flexural cracks and extensive spalling of concrete at the hinge zone was observed during the motion amplitude B. The spalled region raised to a height of 10 in on the north side (Figure 3.34(a)). Crushing of concrete at the base of the column was observed on the north and south faces. Three longitudinal bars were visible with core damage beginning to extend beyond them. Buckling of these three longitudinal bars occurred, one on the south side and two on the north side (Figure 3.34(b)). The farthest bar on the south face buckled across a distance of 2 in, starting 
above the footing surface. The bars on the north side buckled across a distance of 6 in, starting at 2 in above the footing surface. Two damage states occurred during motion amplitude B, namely, crushing of concrete and longitudinal bar buckling. It is worth noting that the same motion amplitudes (i.e., A and B), the accumulated damage in column $\mathrm{C} 2 \mathrm{C}-\mathrm{M}$ was greater than in column $\mathrm{C} 1 \mathrm{C}-\mathrm{L}$. This result may be attributed to the longer duration of the Curico record and will be further discussed in the following section.

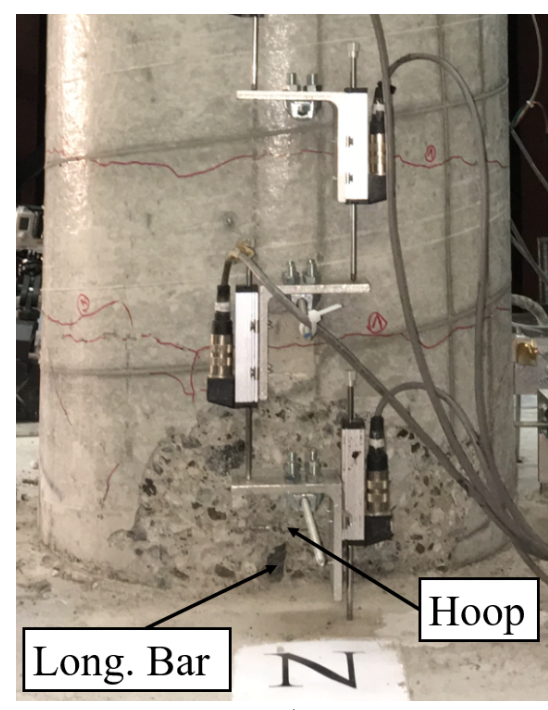

a)

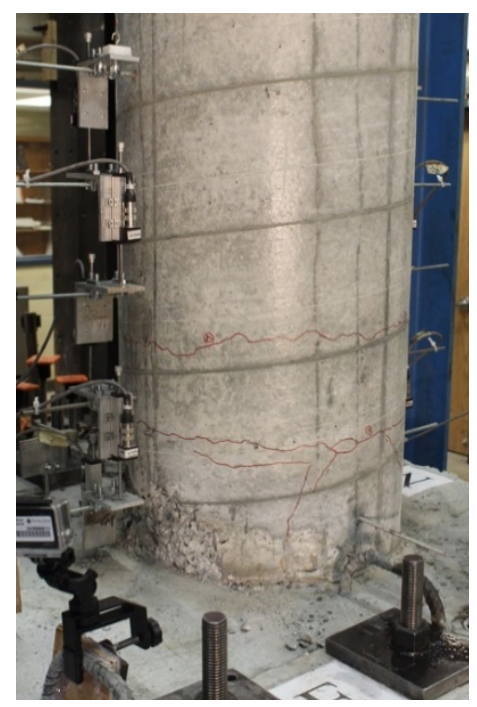

b)

Figure 3.33 Damage state of column C2C-M after applying motion amplitude A. a) North view, b) south-east view

Extensive core damage past the longitudinal bars and fracture of previously buckled reinforcing bars was observed during motion amplitude C (Figure 3.35). Each bar fractured at mid-height of the buckle length. Even though column C1C-L collapse during amplitude $\mathrm{C}$ of the Capitola record, column $\mathrm{C} 2 \mathrm{C}-\mathrm{M}$ did not. Nevertheless, the scale factor associated with motion amplitude $\mathrm{C}$ had no specific target displacement ductility, and therefore comparisons between specimens for this motion amplitude are 
meaningless. However, the mode of failure was different under the subduction ground motion, since the damage state of bar fracture was observed. This result will also be further discussed in the following section.

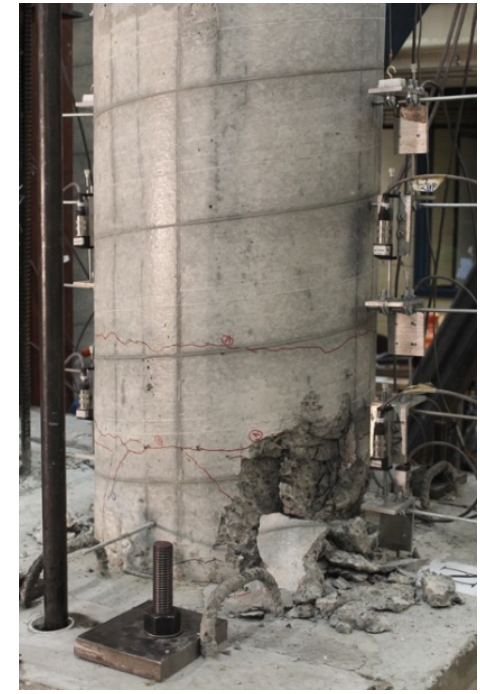

a)

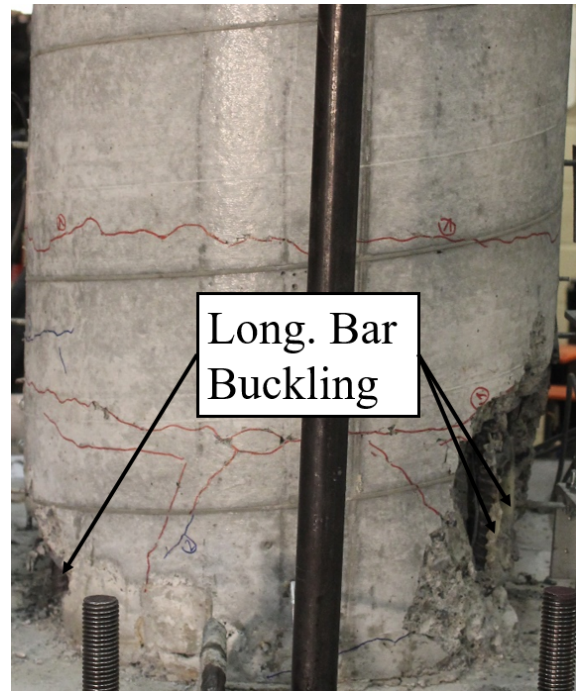

b)

Figure 3.34 Damage state of column C2C-M after applying motion amplitude B. a) North-east view, b) east view

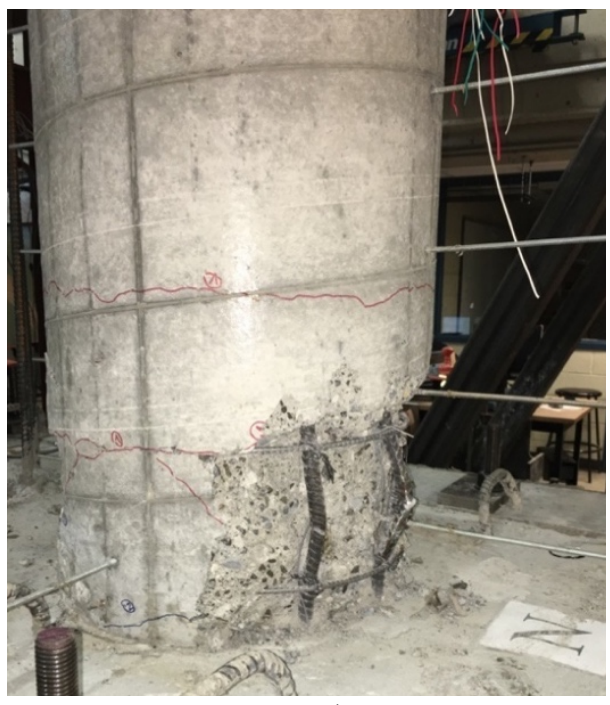

a)

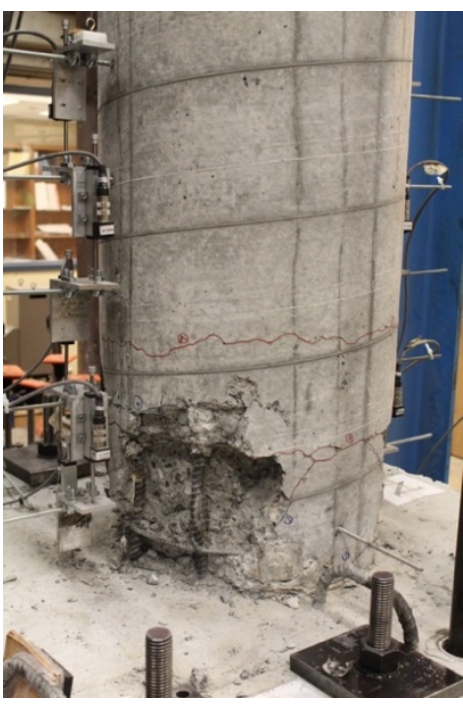

b)

Figure 3.35 Damage state of column C2C-M after applying motion amplitude C. a) North-east view, b) south-east view 


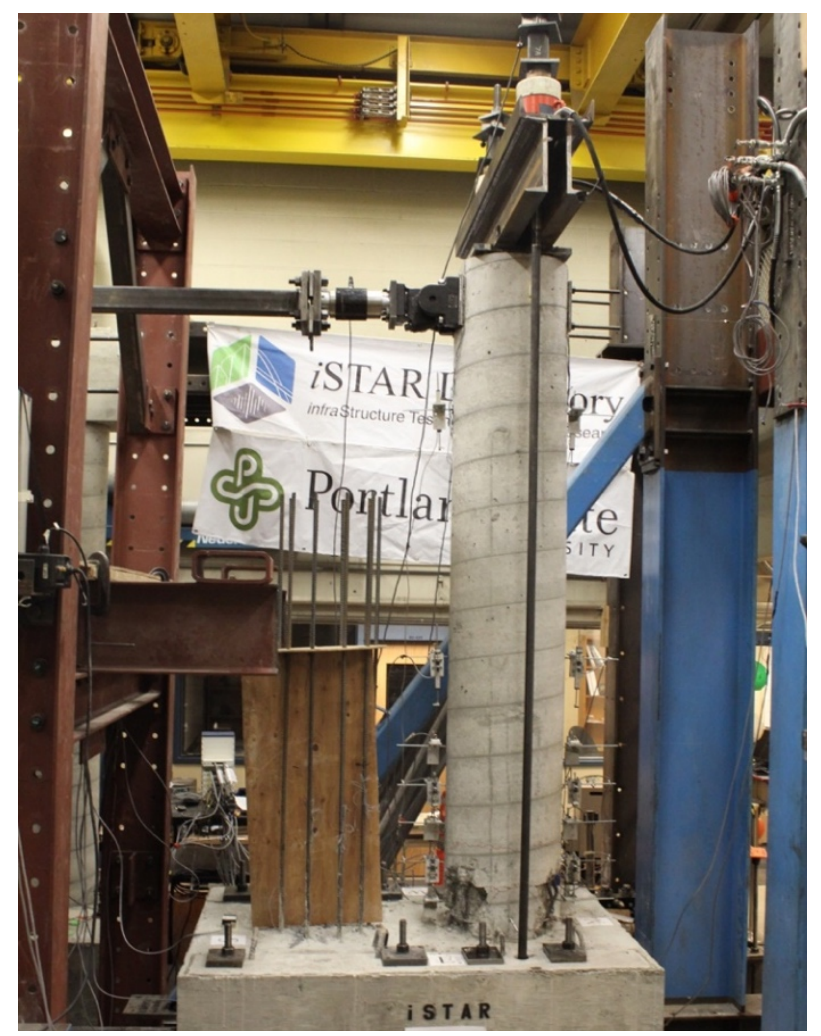

Figure 3.36 Final state of column C2C-M

As expected for a cantilever column, the damage was fully localized to the lower part of the column in the plastic hinge region. Figure 3.33 through Figure 3.35 show the different visual damage states for Column C2C-M after each ground motion amplitude. Figure 3.36 shows the specimen at completion of testing.

\subsubsection{Force-Displacement Relationship}

The measured hysteresis curves for column C2C-M for all ground motion amplitudes are shown in Figure 3.37 through Figure 3.39. Damage states are also identified on the force-displacement response histories. As was mentioned previously, the specimen was loaded in the north-south direction. This means that positive displacement 
direction corresponds to the north direction, and negative displacement direction is towards the south direction.

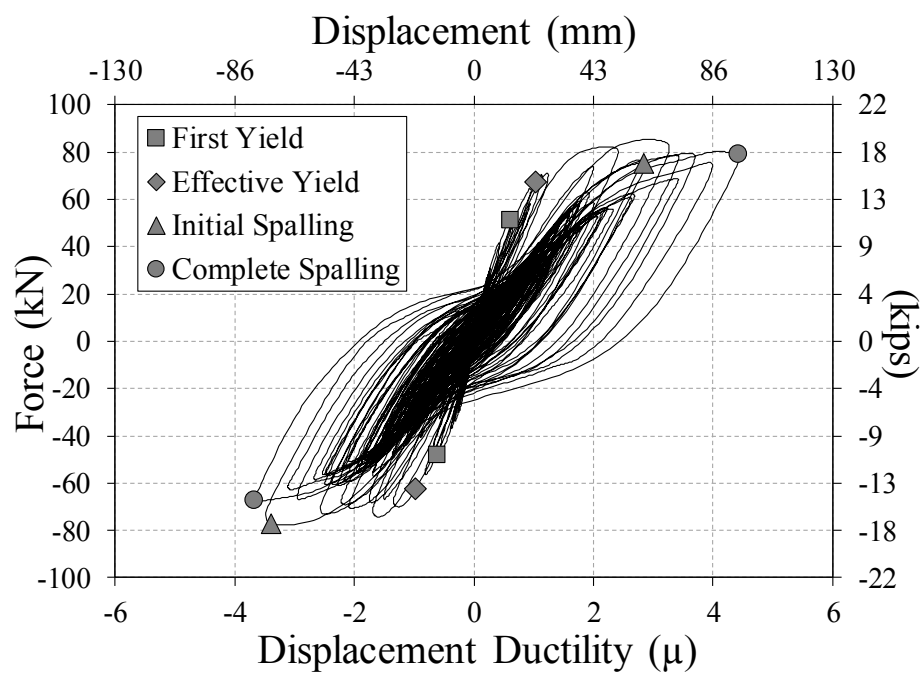

Figure 3.37 Hysteretic response for column C2C-M during motion amplitude A

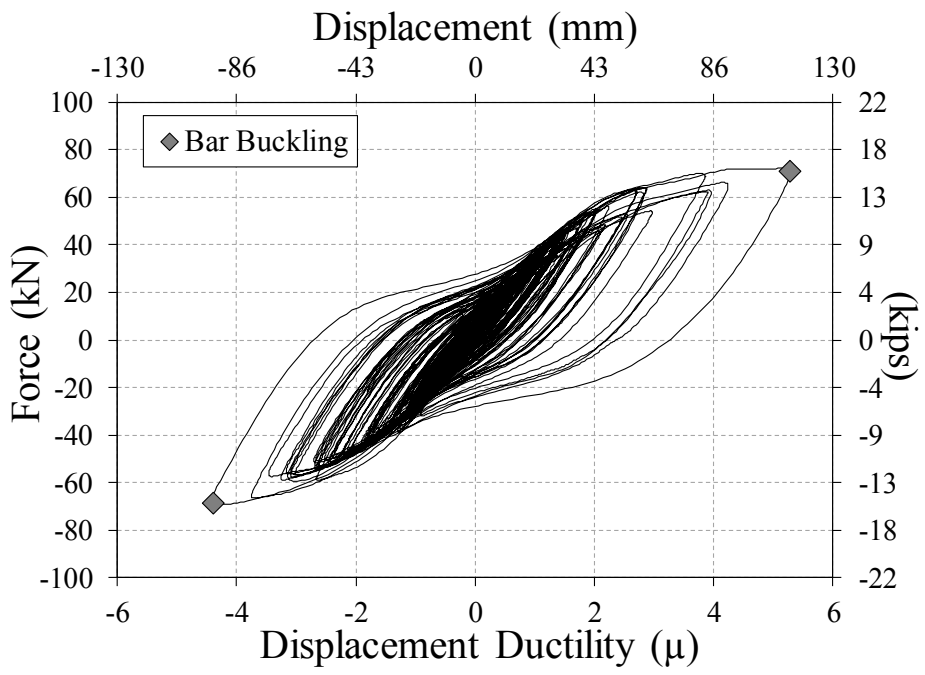

Figure 3.38 Hysteretic response for column C2C-M during motion amplitude B

Based on the experimental results, column $\mathrm{C} 2 \mathrm{C}-\mathrm{M}$ showed a hysteretic response typical of flexural behavior. For motion amplitude A, degradation of column stiffness was exhibited after first bar yielding. After the first motion amplitude, lateral strength of Column $\mathrm{C} 2 \mathrm{C}-\mathrm{M}$ dropped to $86 \%$ of the peak load due to the complete spalling of 
concrete cover and damage extending to the core concrete (Figure 3.37). For motion amplitude B (Figure 3.38), the specimen was not able to reach the target displacement ductility of six due to the clear degradation in column stiffness. Column's lateral strength dropped below $80 \%$ of the peak load due to longitudinal bar buckling and subsequent core concrete damage. The primary mode of failure was flexural hinging failure with buckling of longitudinal reinforcement, due to the low level of transverse reinforcement in the hinge zone. As in $\mathrm{C} 1 \mathrm{C}$-L, motion amplitude $\mathrm{C}$ was performed despite the extent of damage and caused the complete failure of the specimen. Column's lateral strength dropped to $70 \%$ of the peak load due to the rupture of previously buckled reinforcing bars and core concrete crushing (Figure 3.39). Despite the design deficiencies of column C2C$\mathrm{M}$, namely, low ratio of transverse reinforcement and no seismic detailing, this column exhibited a moderate ductile behavior and energy dissipation.

The maximum displacement for each earthquake motion amplitude, and its corresponding drift ratio and ductility level are presented Table 3.8. Reported displacement ductility levels are based on the effective yield displacement, which was found to be $0.85 \mathrm{in}$. This value was computed using the reduce stiffness method assuming an elasto-plastic idealization. These values are the peak displacement values and do not correspond to the peak lateral force values, which are also listed in Table 3.8. The displacement history of column $\mathrm{C} 2 \mathrm{C}-\mathrm{M}$ is shown in Figure 3.40. It is worth nothing that first bar fracture did not occur at the maximum absolute displacement demand, therefore the failure of column $\mathrm{C} 2 \mathrm{C}-\mathrm{M}$ can be attributed to the large number of applied cycles imposed by subduction zone ground motions. On the other hand, it is seen in Figure 3.40 
and Table 3.8 that column $\mathrm{C} 2 \mathrm{C}-\mathrm{M}$ experienced little residual displacement for all the motion amplitudes. Final residual displacement after motion amplitude $\mathrm{C}$ was $0.4 \mathrm{in}$.

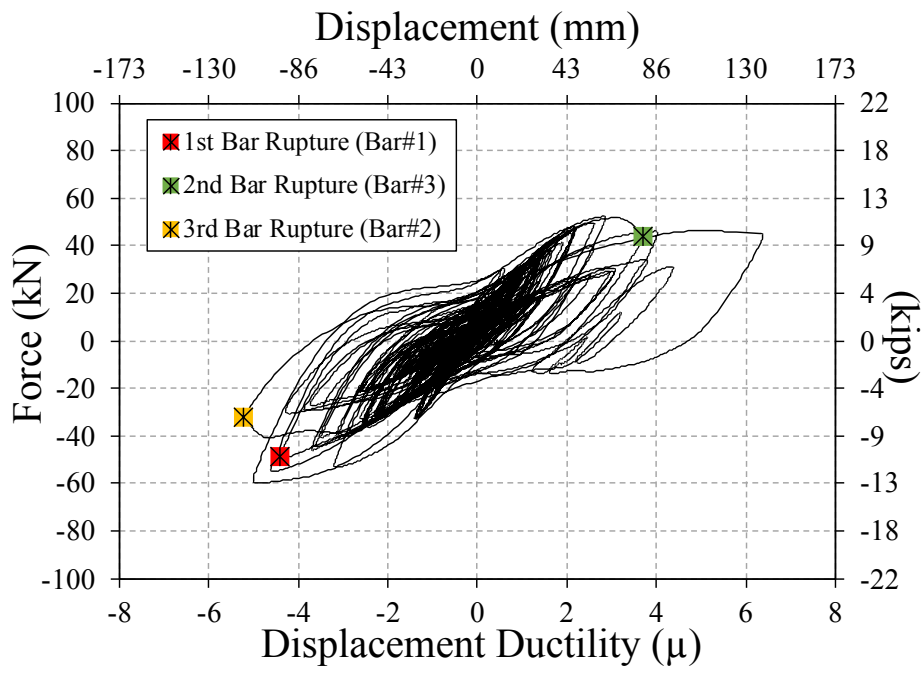

Figure 3.39 Hysteretic response for column C2C-M during motion amplitude C

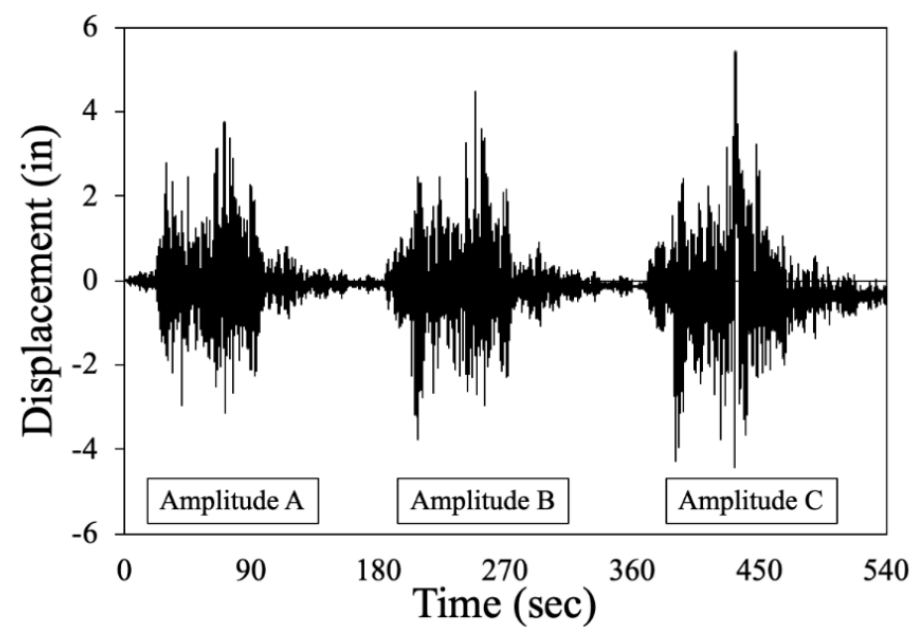

Figure 3.40 Column displacement history for column C2C-M 
Table 3.8 Summary of the measured performance for C2C-M

\begin{tabular}{c|c|c|c}
\hline Property & Amplitude A & Amplitude B & Amplitude C \\
\hline $\begin{array}{c}\text { Max. Displacement } \\
\text { in. }(\mathrm{mm})\end{array}$ & $3.8(96.3)$ & $4.5(114.0)$ & $5.4(137.8)$ \\
\hline $\begin{array}{c}\text { Max. Drift Ratio } \\
(\%)\end{array}$ & 3.95 & 4.68 & 6.35 \\
\hline $\begin{array}{c}\text { Max. Displacement } \\
\text { Ductility Level }(\mu)\end{array}$ & 4.5 & 5.3 & 5.65 \\
\hline $\begin{array}{c}\text { Residual } \\
\text { Displacement in. } \\
(\mathrm{mm})\end{array}$ & $0.02(0.5)$ & $0.15(3.8)$ & $0.40(10.2)$ \\
\hline $\begin{array}{c}\text { Max. Base Shear } \\
\text { kips }(\mathrm{kN})\end{array}$ & $19.2(85.3)$ & $16.2(72.1)$ & $13.5(52.4)$ \\
\hline
\end{tabular}

\subsubsection{Dynamic Properties}

Low-level white noise signals, as well as pulse tests, were performed before and in between earthquake motion amplitudes to monitor the change in the dynamic properties due to damage progression in the specimen. Natural periods were determined from Fourier analysis of the accelerations measured at the top of the column. The damping ratio was calculated from the free vibration portion of the accelerograms recorded at the top of the specimen using the decrement logarithmic method. Since the free-vibration portion of the accelerogram does not have a perfect exponential decay, the accelerogram was divided into various intervals for which a damping ratio was calculated. Then, a mean value was calculated for the damping ratio. A summary of the computed periods and mean damping ratio values and standard deviations are presented in Table 3.9. From the results, it is clear that the computed natural periods were lengthened with the increased level of excitation, what confirmed the deterioration of column stiffness. It can be seen the elongated period after motion amplitude B is almost 
twice the initial cracked period of the column. The variation in damping followed the same trend, i.e., it increased with the progression of the damage from $1.9 \%$ to $6.2 \%$. In general, good agreement was observed between the results of both tests.

Table 3.9 Measured dynamic properties for C2C-M

\begin{tabular}{c|c|c|c|c}
\hline \multirow{2}{*}{ Sequence } & \multicolumn{2}{|c|}{ Period, $T_{n}(\mathrm{sec})$} & \multicolumn{2}{c}{ Damping ratio, $\zeta(\%)$} \\
\cline { 2 - 5 } & Pulse test & W-N random test & $\mu_{X}$ & $\sigma_{X}$ \\
\hline Initial & 0.35 & 0.35 & 1.9 & 0.32 \\
\hline amplitude A & 0.58 & 0.59 & 4.5 & 0.16 \\
\hline amplitude B & 0.60 & 0.64 & 4.8 & 0.33 \\
\hline amplitude C & 0.83 & 0.87 & 6.2 & 0.72 \\
\hline
\end{tabular}

\subsubsection{Strains}

Figure 3.41 through Figure 3.44 show some of the recorded steel strain histories during earthquake motion amplitudes $\mathrm{A}$ and $\mathrm{B}$ for the tested specimen. This column was subjected to a larger number of strain cycles as compared to C1C-L. Based on the measured values, peak steel strain profiles were derived and plotted (Figure 3.45) for Specimen $\mathrm{C} 2 \mathrm{C}-\mathrm{M}$ along the plastic hinge. In these figures, some strain measurements are missing due to either damage during concrete pouring or failure during testing. From the Peak steel strain profile, it is seen that the strains were larger that the yield strain in the plastic hinge region. A maximum strain of $0.026 \mathrm{in} /$ in was measured in the south rebar during motion amplitude A, which corresponds to 15 times the yield strain $\left(\varepsilon_{y}\right)$. In general, strains were slightly larger in the south side of the column. 


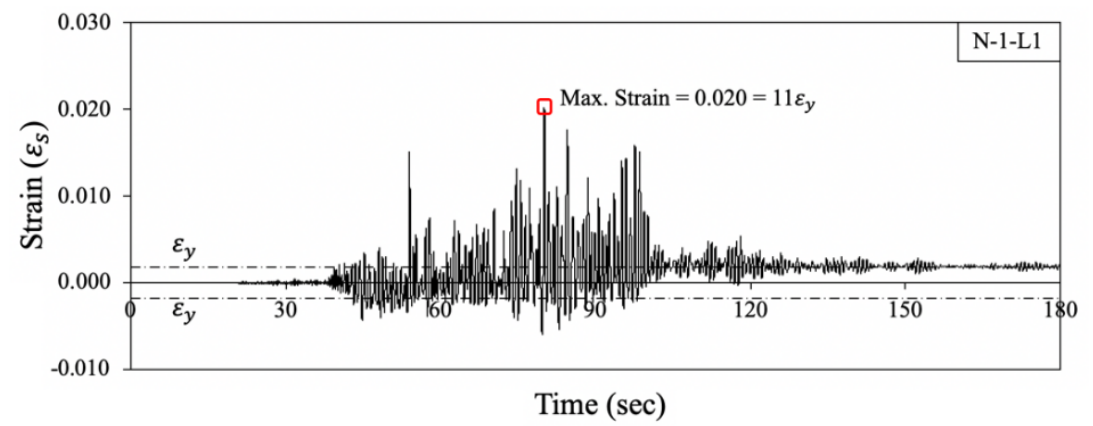

Figure 3.41 Steel strain history for strain gauge N-1-L2 for column C2C-M during motion amplitude $\mathrm{A}$

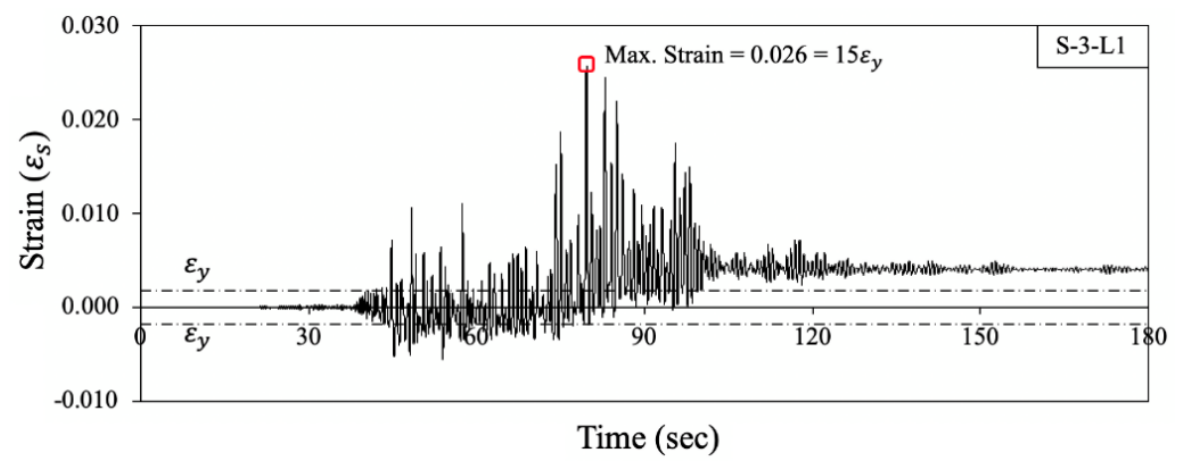

Figure 3.42 Steel strain history for strain gauge S-3-L1 for column C2C-M during motion amplitude A

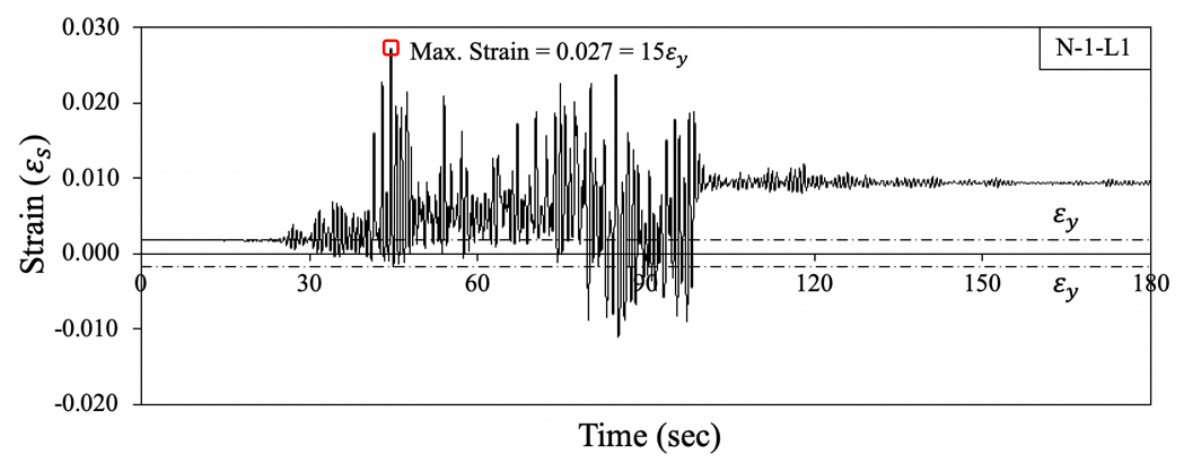

Figure 3.43 Steel strain history for strain gauge N-3-L2 for column C2C-M during motion amplitude B 


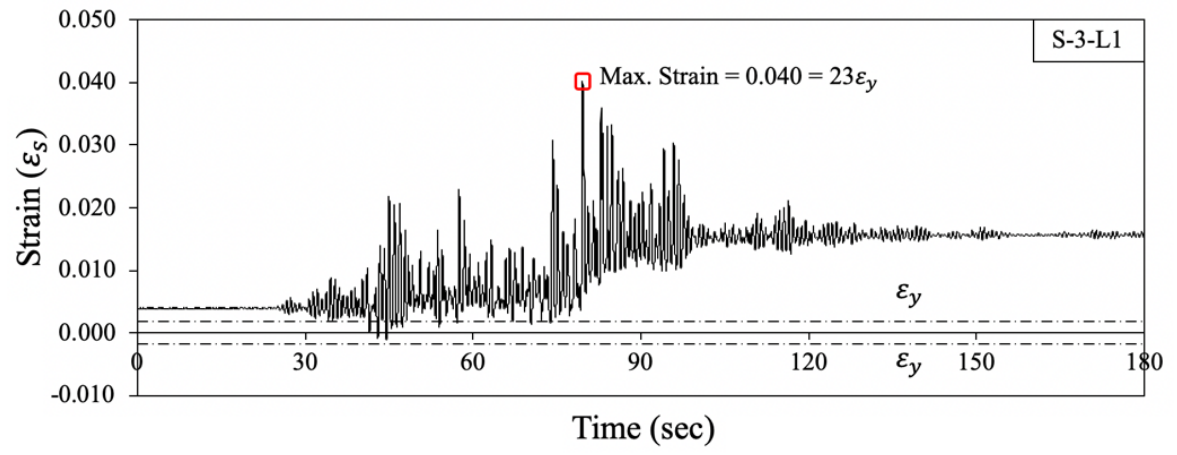

Figure 3.44 Steel strain history for strain gauge S-3-L1 for column C2C-M during motion amplitude B

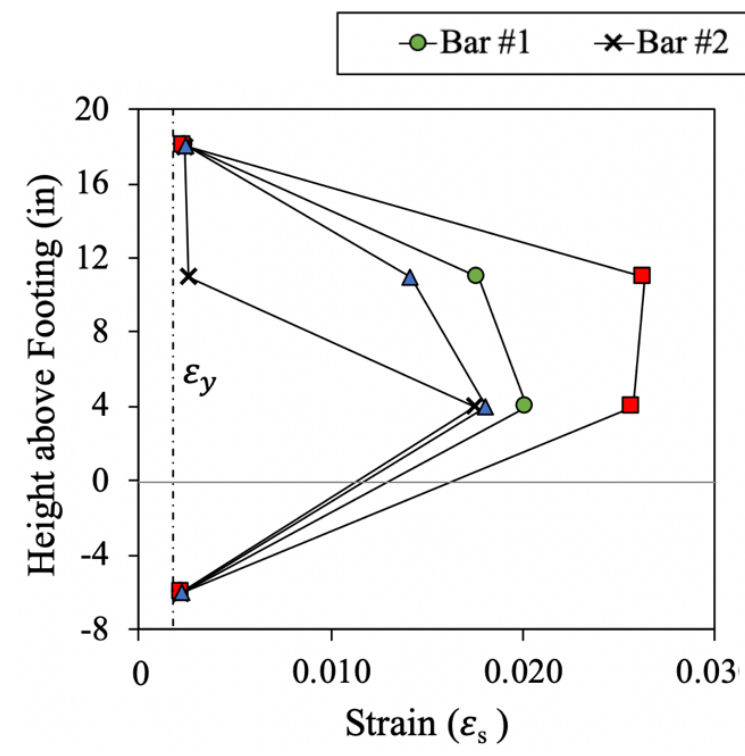

a)

\section{$\square-$ Bar \#3 $\triangle$-Bar\#4}

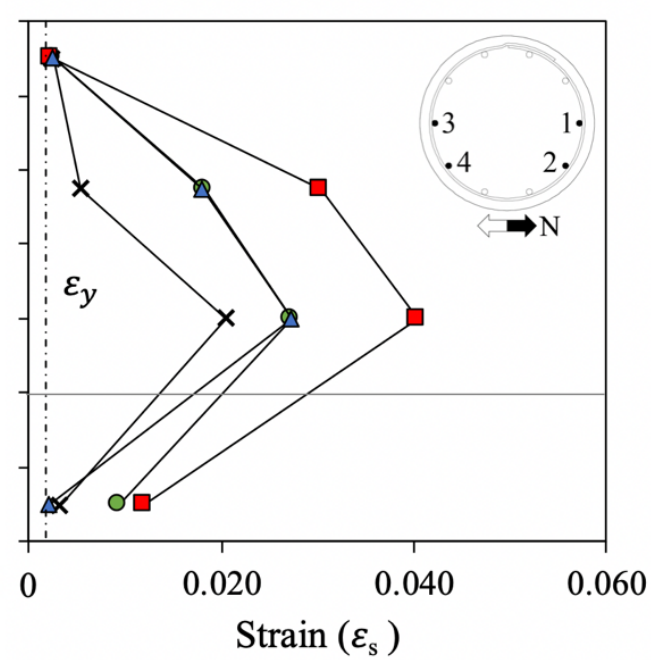

b)

Figure 3.45 Peak steel strain profile for column C2C-M. a) Amplitude A, b) Amplitude B

\subsubsection{Curvature}

As mentioned before, linear variable displacement transducers (LVDTs) were used to measure the average curvature along the height of the column. The peak curvature profile along the height is shown in Figure 3.46 for each motion amplitude. The points in the curvature profiles represent the curvature measured at the specific location and in correspondence to the maximum and minimum peak values of column 
displacement. The curvature profiles in each direction were symmetric, in agreement with the symmetry of the ground motion record. Also, as it was expected for columns tested on single curvature, large curvature values were measured at the base of the column, where the moments were larger and induced substantial nonlinear deformation. It is shown in the figure that the desired behavior was achieved in the plastic hinge region where the visible damage occurred (concrete cracks, spalling, core crushing, etc.)

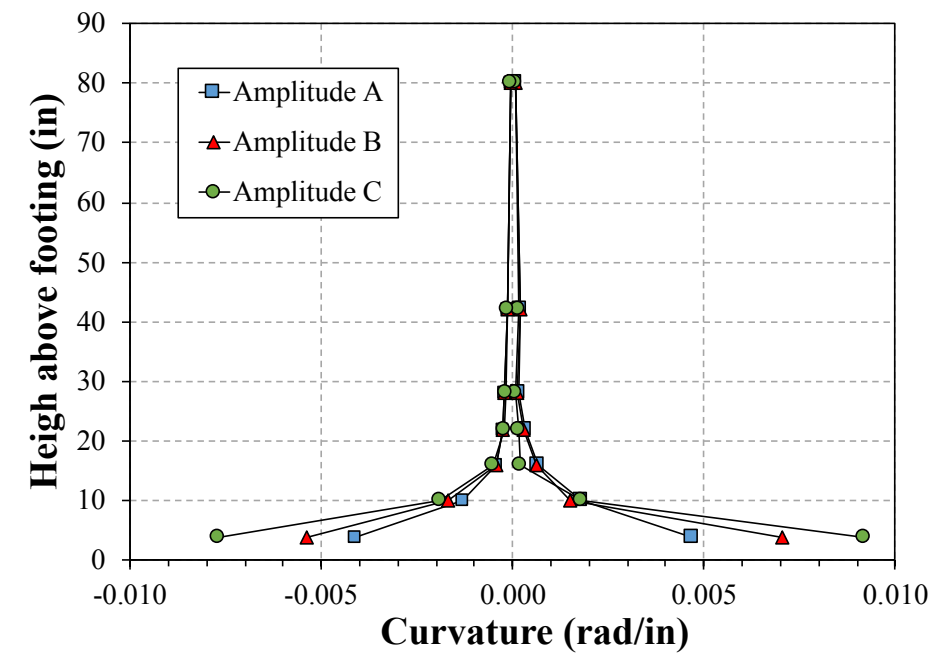

Figure 3.46 Average curvature profiles in column C2C-M

\subsubsection{Axial Forces}

Although the experiments were designed to keep the same level of axial force during the test sequence, some variations were observed in each earthquake motion amplitude during testing. The axial force history for specimen C1C-L is shown in Figure 3.47. The target force was 75 kips $(334 \mathrm{kN})$ for motion amplitude $\mathrm{A}$ and $\mathrm{B}$, and 60 kips $(267 \mathrm{kN})$ for motion amplitude $\mathrm{C}$. The maximum deviation from the target axial force was $-2.3 \%$ and $+23.2 \%$ in motion amplitude $\mathrm{A},-0.2 \%$ and $+22.8 \%$ in motion amplitude $\mathrm{B}$, and $-1.5 \%$ and $+27 \%$ in motion amplitude $\mathrm{C}$. Even though there were fluctuations in 
the applied axial force during testing, they were smaller than the ones observed for column C1C-L. The variations of the axial force may be attributed to the change in axial loads in the treaded rods as the specimen displaced. Normally, the accumulators compensate for this, but the hinging action of the load transfer beam created a less stiff axial load system. The less stiff system caused the hydraulic ram to displace further and faster than its ability to maintain constant axial load.

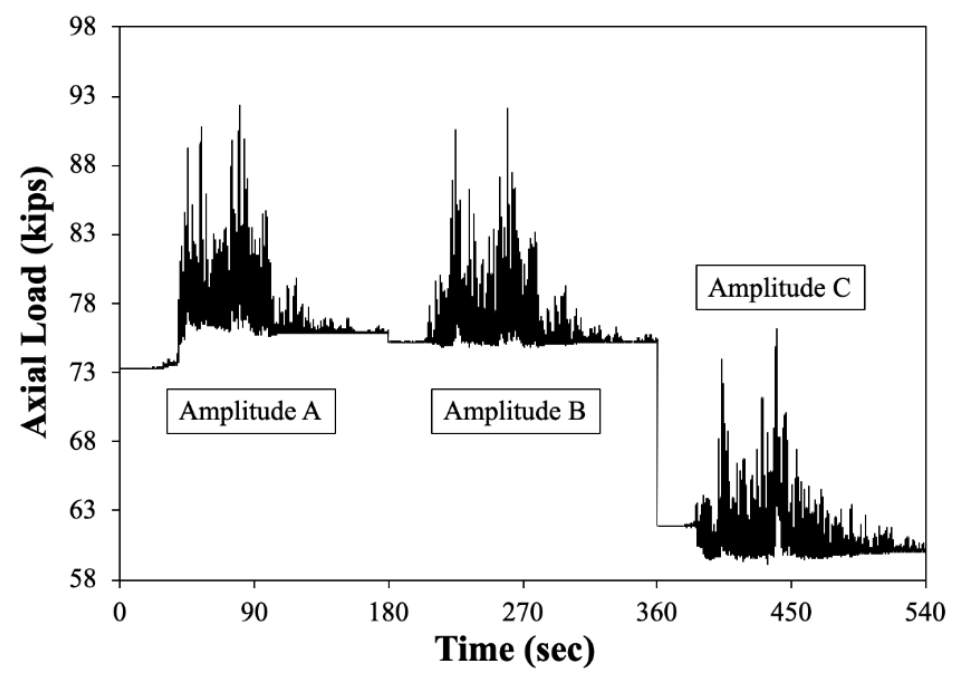

Figure 3.47 Axial force history for column C2C-M

\subsubsection{Shake Table Output Motions}

Comparison between the input and output motions is also presented for the Curico $\mathrm{X}$ record. For the input motion applied column $\mathrm{C} 2 \mathrm{C}-\mathrm{M}$, small differences between the target and achieved accelerations reproduced by the shake table were observed despite the fine-tuning applied to the system. Comparison of the target and recorded time history accelerations for each motion amplitude are shown in Figure 3.48(a). Table 3.10 shows the target and achieved values of peak table acceleration (PGA), velocity (PGV), and displacement (PGD) for each motion amplitude. In addition to the peak responses 
comparison, $5 \%$ damped elastic acceleration response spectrums were calculated from the target and achieved acceleration signals for each motion amplitude, as depicted in Figure 3.48(b). Also, it is shown in the same figures the measured initial and final period of the specimen. Although there are variations in the target and achieved elastic response accelerations for all the motions, these discrepancies are more important at the low and high period portion of the spectrum. The impact of these discrepancies is not significant since the potential specimen's response is in between that range of periods as it is shown in the spectrums. Therefore, the table performance is judged as satisfactory for the period range of interest.

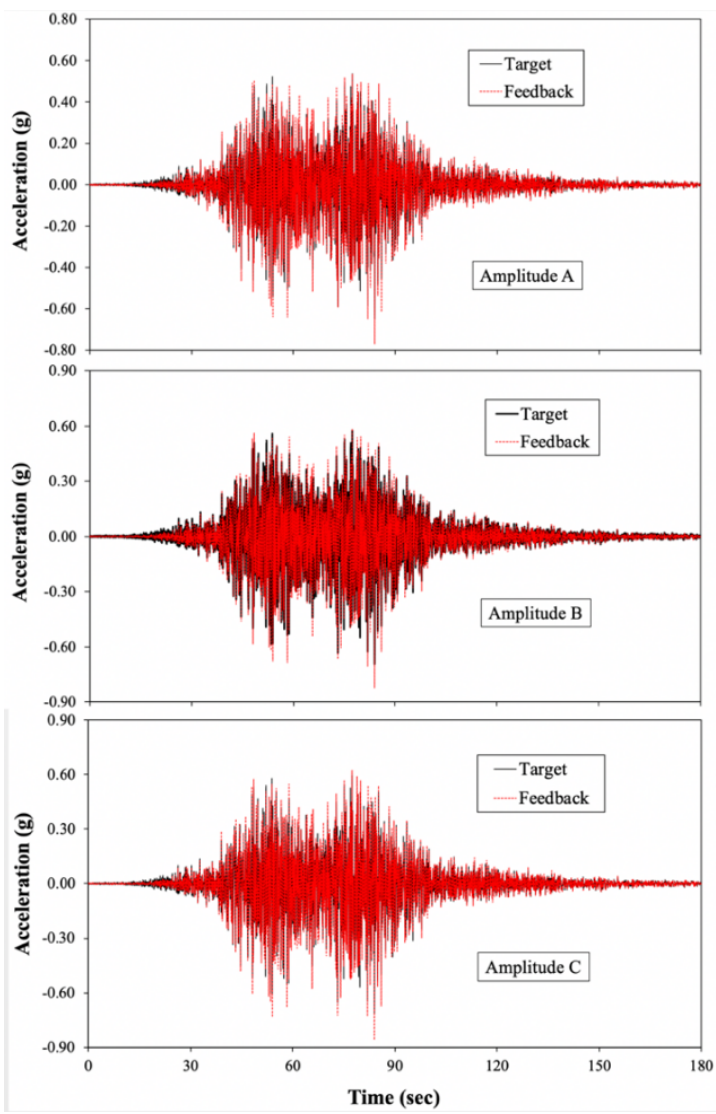

a)

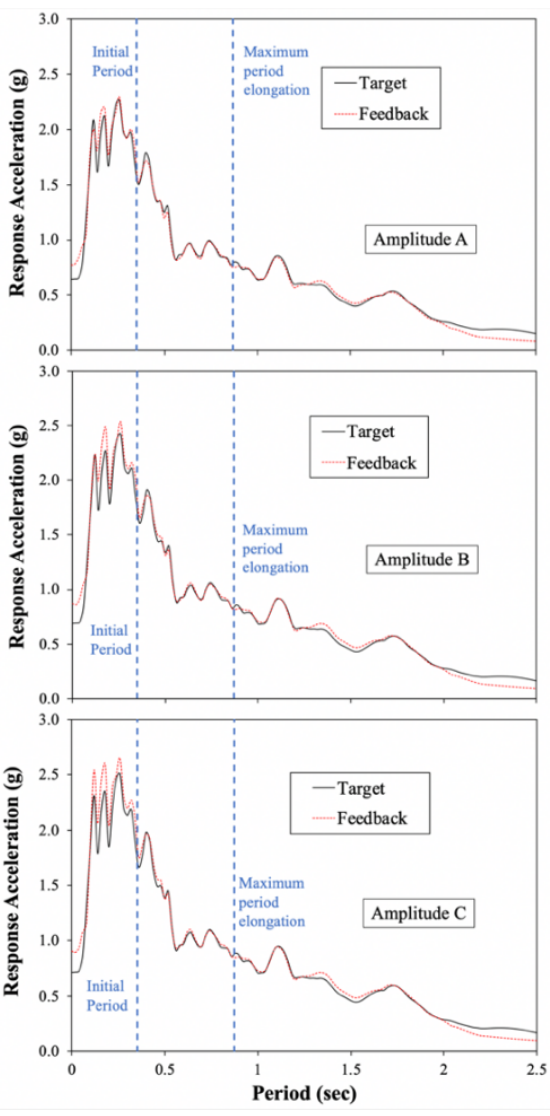

b)

Figure 3.48 Shake table performance for Curico X. a) Acceleration time histories, b) 5\% damped elastic response spectra 
Table 3.10 Target and achieved peak table responses for Curico X

\begin{tabular}{c|c|c|c|c|c|c}
\hline \multirow{2}{*}{ Amplitude } & \multicolumn{2}{|c|}{ PGA $(\mathrm{g})$} & \multicolumn{2}{c|}{ PGV (in/sec) } & \multicolumn{2}{c}{ PGD (in) } \\
\cline { 2 - 7 } & Target & Achieved & Target & Achieved & Target & Achieved \\
\hline A & 0.65 & 0.77 & 20.01 & 18.70 & 3.73 & 3.25 \\
\hline B & 0.69 & 0.83 & 21.41 & 20.27 & 4.00 & 3.48 \\
\hline C & 0.72 & 0.86 & 22.17 & 21.07 & 4.14 & 3.62 \\
\hline
\end{tabular}

\subsubsection{Column C3C-T}

Column C3C-T was the third column tested. It was subjected to the Iwaki Y record from the 2011 Tohoku earthquake (subduction). Test results and discussion are presented in the following subsections.

\subsubsection{Progression of Observed Damage}

The specimen was first subjected to amplitude A of the selected ground motion. During this amplitude motion, the damage on the column was characterized by horizontal cracks developed all around the circumference of the column at the first levels of transverse reinforcement. Three major horizontal cracks at 2 in, 8 in, and 14 in above the column base were observed during testing but were closed after shaking. The specimen also exhibited minimum vertical cracking in the plastic hinge region. Following first bar yielding, initial spalling of concrete cover occurred at motion amplitude A. The spalled height extended vertically 4.5 in from the interface, in the north and south faces of the specimen (Figure 3.49). Propagation of flexural cracks and spalling of concrete at the hinge zone was observed during the motion amplitude $\mathrm{B}$. The spalled region raised to a height of 8 in on the south side (Figure 3.50(b)). Crushing of concrete at the base of the column was observed on the north and south faces. The farthest longitudinal bars were visible on the north and south side, although no significant core damage had happened. 
Only the exposed bar at the south face buckled across a distance of 6 in, starting at 2 in above the footing surface (Figure 3.50(b)). Two failure modes occurred during this motion, namely, crushing of concrete and longitudinal bar buckling.

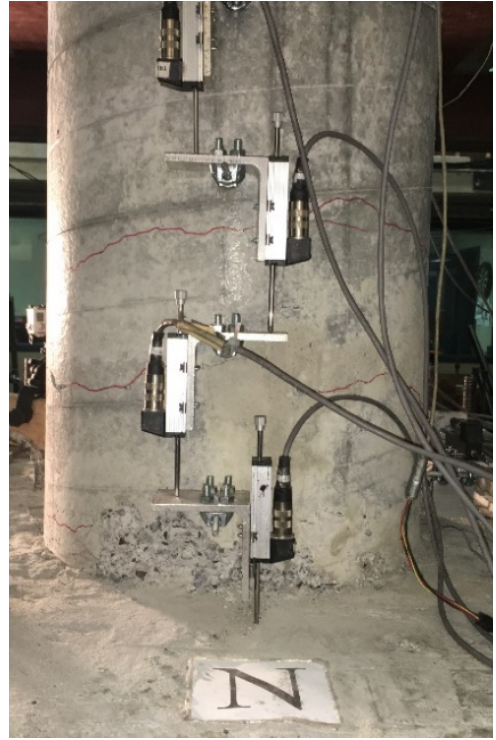

a)

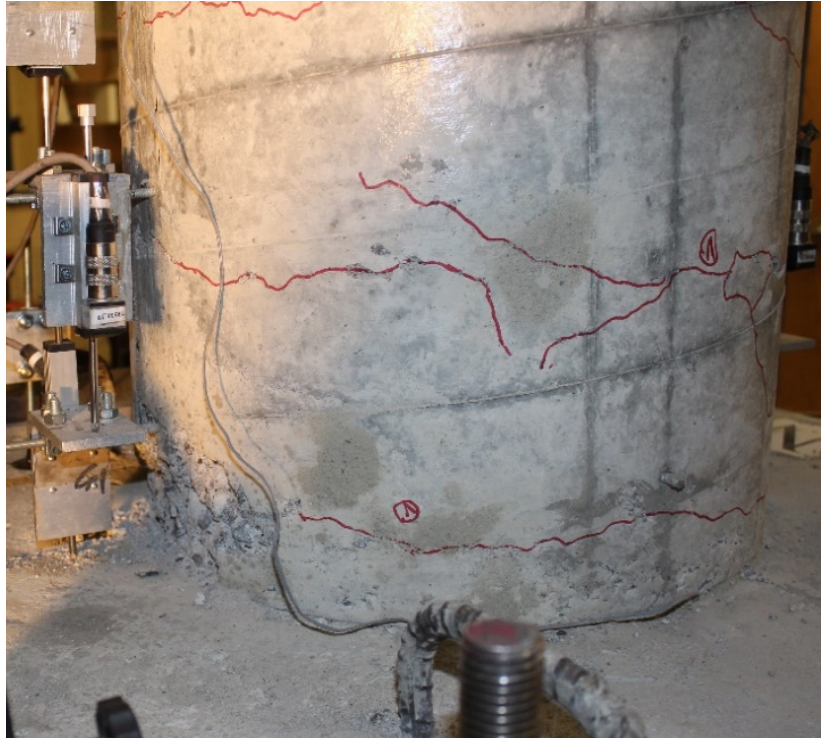

b)

Figure 3.49 Damage state of column C3C-T after applying motion amplitude A. a) North view, b) south-east view

Column C3C-T reached its final damage state during motion amplitude $\mathrm{C}$, as depicted in Figure 3.51. Extensive core damage past the longitudinal bars was observed on the south side. Fracture of the extreme longitudinal bar on the south side occurred during this motion. The two adjacent longitudinal bars on the south side were buckled across a distance of 6 in, starting at 2 in above the footing surface. Since the north side of the column had less visible damage than the south side, it was not possible to see if the longitudinal bars on the north side were buckled. The ultimate mode of failure was crushing of concrete, longitudinal bar buckling and longitudinal bar fracture. As observed in column $\mathrm{C} 2 \mathrm{C}-\mathrm{M}$, the mode of failure was different under the subduction ground motion 
as compared to the crustal demand. This result will be further discussed in the following section.

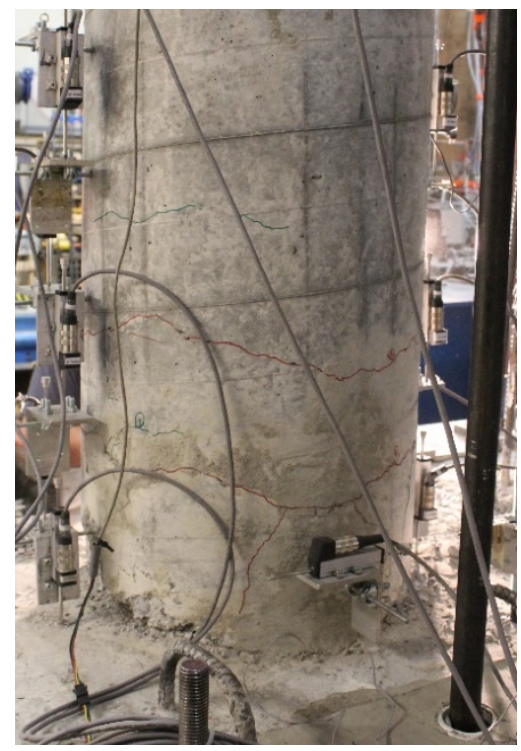

a)

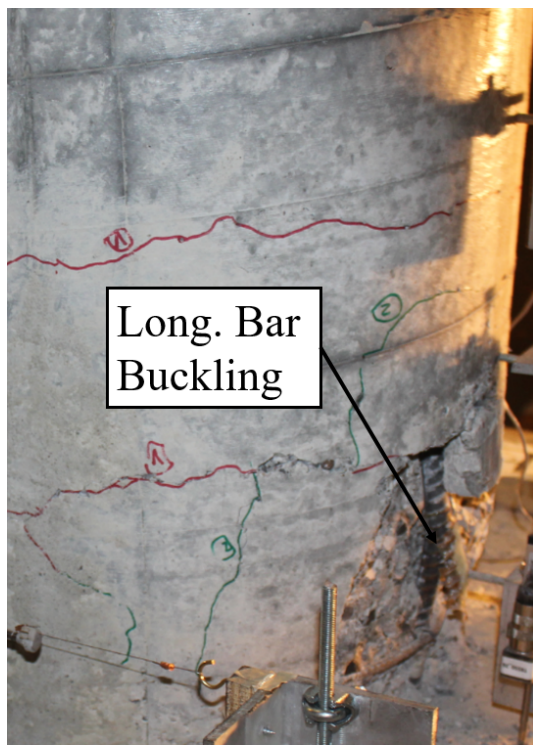

b)

Figure 3.50 Damage state of column C3C-T after applying motion amplitude B. a) North-east view, b) south-west view

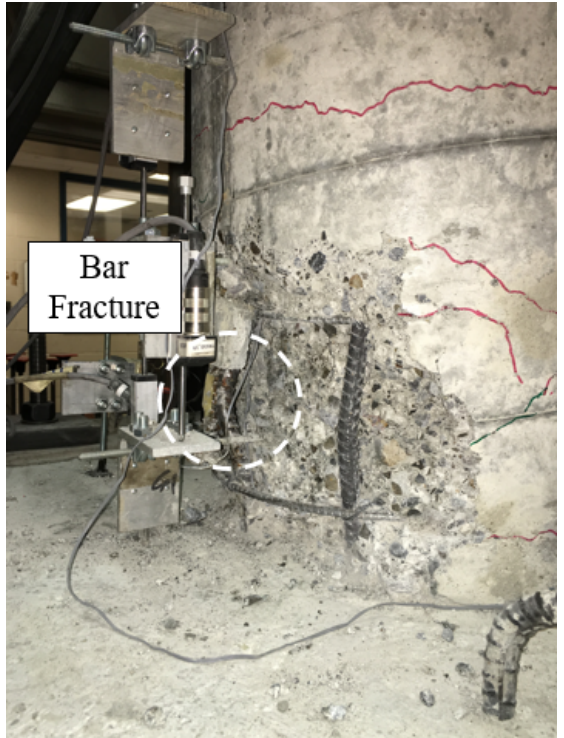

a)

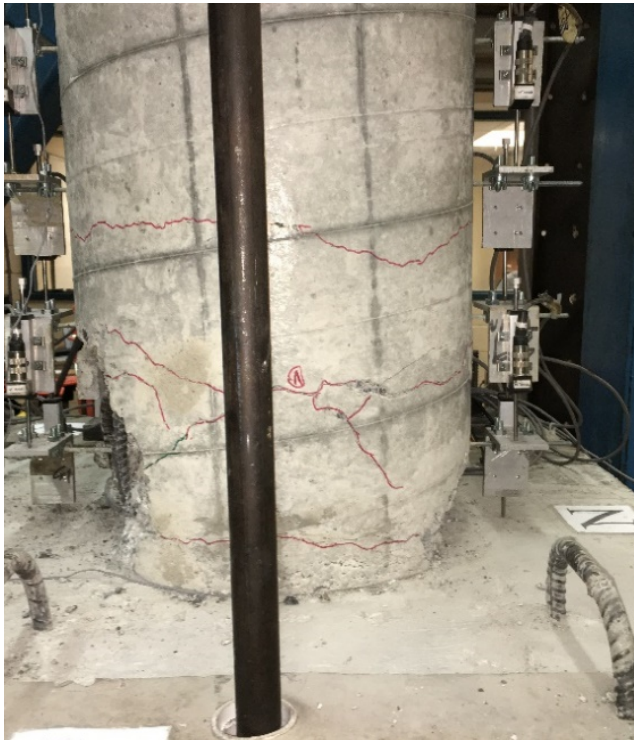

b)

Figure 3.51 Damage state of column C3C-T after applying motion amplitude C. a) South-east view, b) east view 
As expected for a cantilever column, the damage was fully localized to the lower part of the column in the plastic hinge region. Figure 3.49 through Figure 3.51 show the different visual damage states for Column C3C-T after each ground motion amplitude. Figure 3.52 shows the specimen at completion of testing.

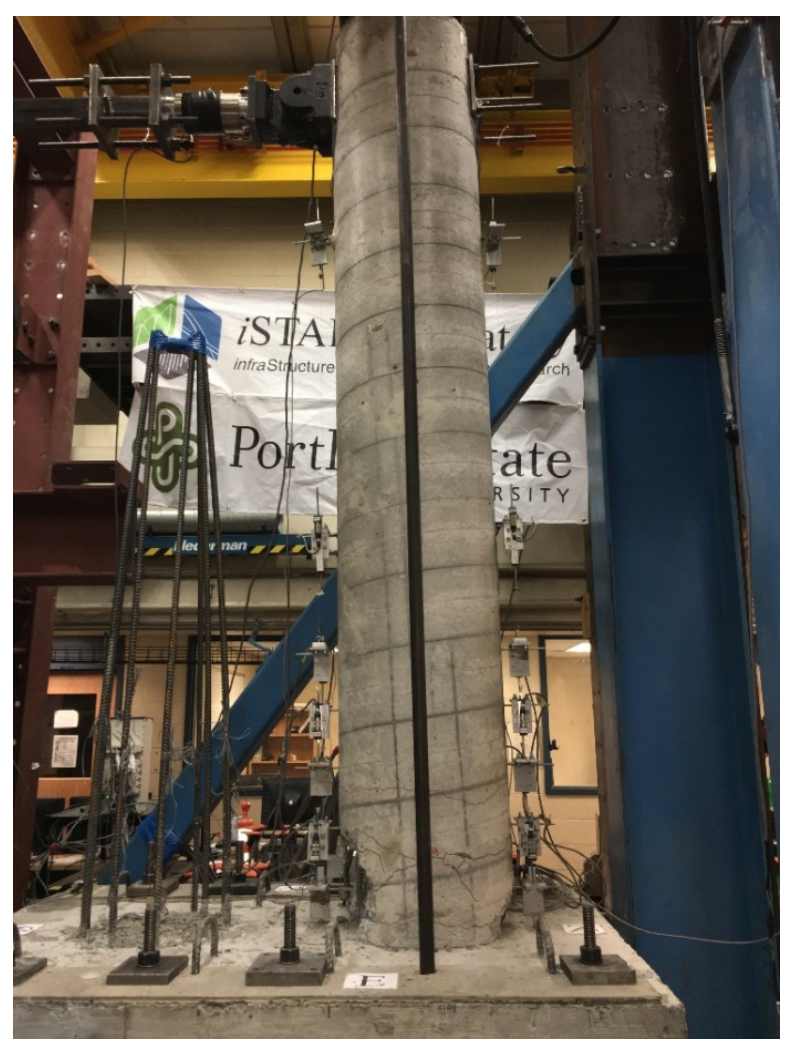

Figure 3.52 Final state of column C3C-T

\subsubsection{Force-Displacement Relationship}

The measured hysteresis curves for column C3C-T for all ground motion amplitudes are shown in Figure 3.53 through Figure 3.55. Damage states are also identified on the force-displacement response histories. As was mentioned previously, the specimen was loaded in the north-south direction. This means that positive displacement 
direction corresponds to the north direction, and negative displacement direction is towards the south direction.

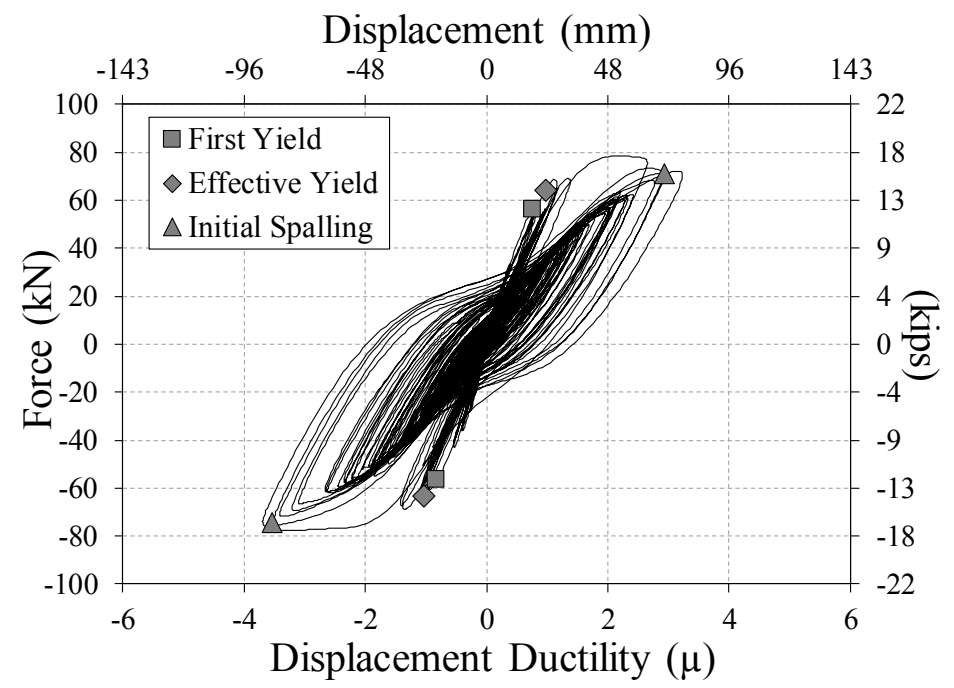

Figure 3.53 Hysteretic response for column C3C-T during motion amplitude A

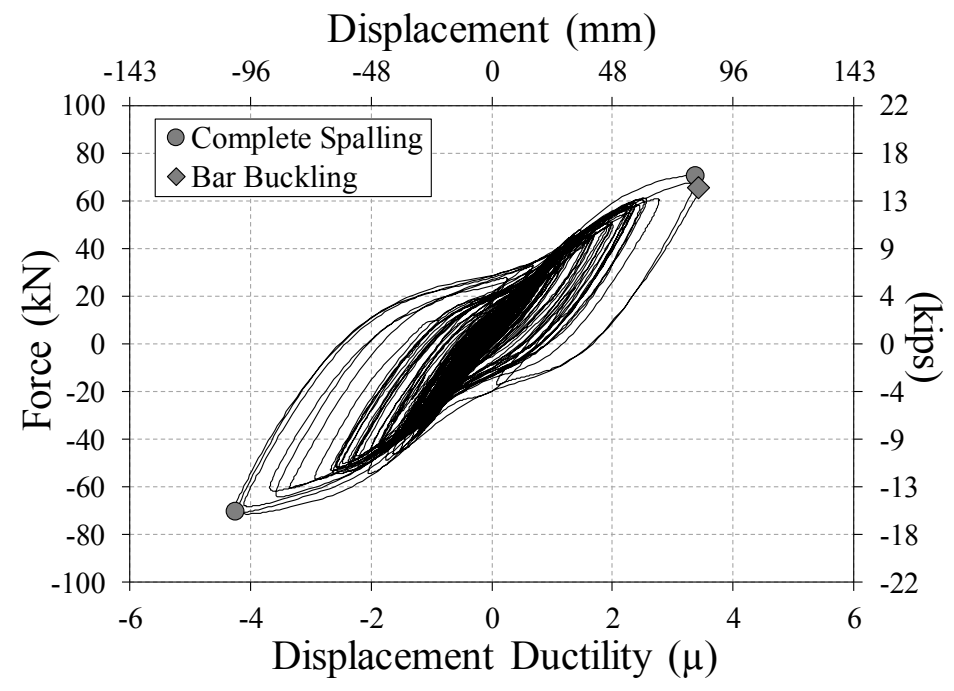

Figure 3.54 Hysteretic response for column C3C-T during motion amplitude B

Based on the experimental results, column $\mathrm{C} 3 \mathrm{C}-\mathrm{T}$ showed a hysteretic response typical of flexural behavior. For motion amplitude A, degradation of column stiffness was exhibited after first bar yielding. After the first motion amplitude, the specimen did not exhibit a significant reduction in its lateral strength (Figure 3.53). For motion 
amplitude B (Figure 3.54), the specimen was not able to reach the target displacement ductility of six due to the clear degradation in column stiffness. Column's lateral strength dropped below $90 \%$ of the peak load due to longitudinal bar buckling. The primary mode of failure was flexural hinging failure with buckling of longitudinal reinforcement, due to the low level of transverse reinforcement in the hinge zone. As in the previous test specimen, motion amplitude $\mathrm{C}$ was performed and caused the complete failure of the specimen. Column's lateral strength dropped to $75 \%$ of the peak load due to the rupture of previously buckled reinforcing bars and core concrete crushing (Figure 3.55). Despite the design deficiencies of column $\mathrm{C} 3 \mathrm{C}-\mathrm{T}$, namely, low ratio of transverse reinforcement and no seismic detailing, this column exhibited a moderate ductile behavior and energy dissipation.

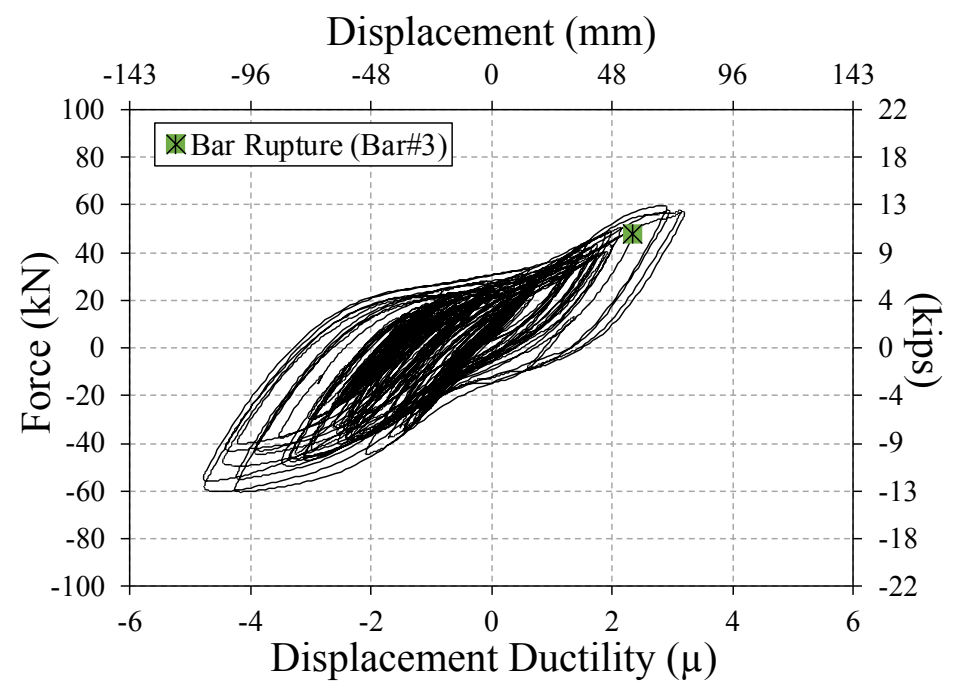

Figure 3.55 Hysteretic response for column C3C-T during motion amplitude C

The maximum displacement for each earthquake motion amplitude, and its corresponding drift ratio and ductility level are presented Table 3.11. These values are the peak displacement values and do not correspond to the peak lateral force values, which 
are also listed in Table 3.11. Reported displacement ductility levels are based on the effective yield displacement, which was found to be $0.94 \mathrm{in}$. This value was computed using the reduce stiffness method assuming an elasto-plastic idealization. It is worth noting that specimen $\mathrm{C} 3 \mathrm{C}-\mathrm{T}$ exhibited a higher tip displacement at first yield, and therefore a higher effective yield displacement, as compare to the other two specimens, which had almost identical initial yield displacement. This difference may indicate that bar slip was larger for C3C-T. The displacement history of column C2C-M is shown in Figure 3.56. It is worth nothing that first bar fracture did not occur at the maximum absolute displacement demand, therefore the failure of column C3C-T can be attributed to the large number of applied cycles imposed by subduction zone ground motions. On the other hand, it is seen in Figure 3.56 and Table 3.11 that column C3C-T experienced a higher residual displacement for all the motion amplitudes, as compared to the other two specimens. This result can be attributed to the longer duration and large number of applied cycles of the Iwaki Y record. Final residual displacement after motion amplitude C was 1.6 in.

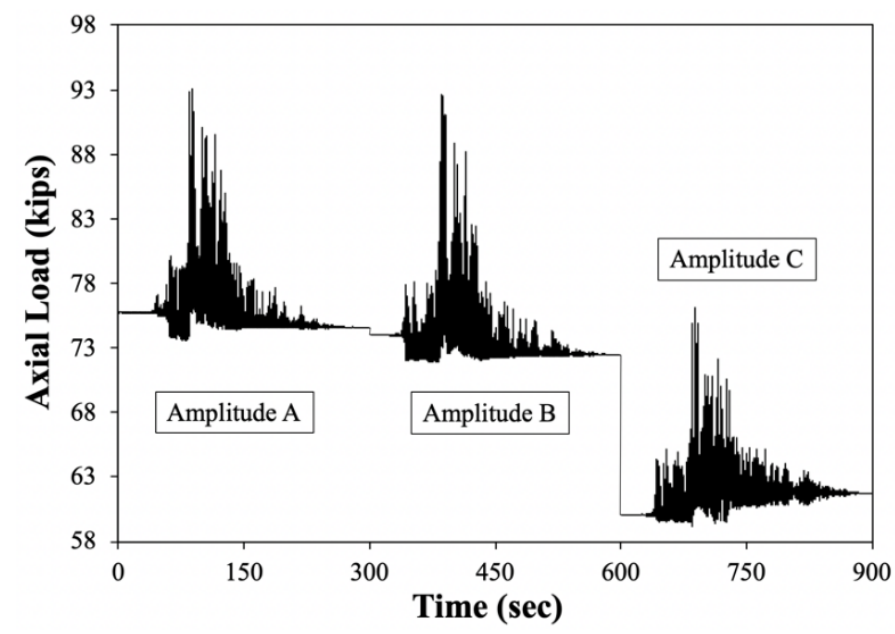

Figure 3.56 Column displacement history for column C3C-T 120 
Table 3.11 Summary of the measured performance for C3C-T

\begin{tabular}{c|c|c|c}
\hline Property & Amplitude A & Amplitude B & Amplitude C \\
\hline $\begin{array}{c}\text { Max. Displacement } \\
\text { in. (mm) }\end{array}$ & $3.5(88.3)$ & $4.0(102)$ & $4.5(114.1)$ \\
\hline $\begin{array}{c}\text { Max. Drift Ratio } \\
(\%)\end{array}$ & 3.62 & 4.18 & 4.68 \\
\hline $\begin{array}{c}\text { Max. Displacement } \\
\text { Ductility Level }(\mu)\end{array}$ & 3.7 & 4.3 & 4.8 \\
\hline $\begin{array}{c}\text { Residual } \\
\text { Displacement in. } \\
(\mathrm{mm})\end{array}$ & $0.16(4.1)$ & $0.28(7.1)$ & $1.56(39.6)$ \\
\hline $\begin{array}{c}\text { Max. Base Shear } \\
\text { kips (kN) }\end{array}$ & $17.6(78.4)$ & $16.2(72.2)$ & $13.6(60.4)$ \\
\hline
\end{tabular}

\subsubsection{Dynamic Properties}

Low-level white noise signals, as well as pulse tests, were performed before and in between earthquake motion amplitudes to monitor the change in the dynamic properties due to damage progression in the specimen. Natural periods were determined from Fourier analysis of the accelerations measured at the top of the column. The damping ratio was calculated from the free vibration portion of the accelerograms recorded at the top of the specimen using the decrement logarithmic method. Since the free-vibration portion of the accelerogram does not have a perfect exponential decay, the accelerogram was divided into various intervals for which a damping ratio was calculated. Then, a mean value was calculated for the damping ratio. A summary of the computed periods and mean damping ratio values and standard deviations are presented in Table 3.12. The uncracked natural period of the column model was 0.39 , which is about $11 \%$ greater than the uncracked natural period of $\mathrm{C} 1 \mathrm{C}-\mathrm{L}$ and $\mathrm{C} 2 \mathrm{C}-\mathrm{M}$. This difference might be attributed to the successive accommodation of the specimen before the actual testing. From the results, it is clear that the computed natural periods were 
lengthened with the increased level of excitation, what confirmed the deterioration of column stiffness. It can be seen the elongated period after motion amplitude B is almost twice the initial cracked period of the column. The variation in damping followed the same trend, i.e., it increased with the progression of the damage from $2.0 \%$ to $4.6 \%$. Comparing to column $\mathrm{C} 2 \mathrm{C}-\mathrm{M}$, period and damping lengthening was less pronounced for C3C-T. However, period lengthening was more pronounced for tests using the subduction earthquakes records. In general, good agreement was observed between the results of both tests.

Table 3.12 Measured dynamic properties for C3C-T

\begin{tabular}{c|c|c|c|c}
\hline \multirow{2}{*}{ Sequence } & \multicolumn{2}{|c|}{ Period, $T_{n}(\mathrm{sec})$} & \multicolumn{2}{c}{ Damping ratio, $\zeta(\%)$} \\
\cline { 2 - 5 } & Pulse test & W-N random test & $\mu_{X}$ & $\sigma_{X}$ \\
\hline Initial & 0.39 & 0.39 & 2.0 & 0.21 \\
\hline amplitude A & 0.60 & 0.60 & 4.1 & 0.28 \\
\hline amplitude B & 0.61 & 0.62 & 4.5 & 0.62 \\
\hline amplitude C & 0.65 & 0.66 & 4.6 & 0.70 \\
\hline
\end{tabular}

\subsubsection{Strains}

Figure 3.57 through Figure 3.60 show some of the recorded steel strain histories during different earthquake motion amplitudes for the tested specimen. This column was subjected to large number of strain cycles compared to $\mathrm{C} 2 \mathrm{C}-\mathrm{M}$. Based on the measured values, peak steel strain profiles were derived and plotted (Figure 3.61) for Specimen $\mathrm{C} 3 \mathrm{C}-\mathrm{T}$ along the plastic hinge. The used strain gauges were able to record most of the strain measurements; however, some stain data is missing due to either damage during concrete pouring or failure during testing. It is seen in the plots that the instrumented bars showed a residual strain equal or greater than the yielding strain for the first motion 
amplitude. Further, from the Peak steel strain profile, it is seen that the strains were larger that the yield strain in the plastic hinge region. A maximum strain of 0.020 in/in was measured in the south rebar during motion amplitude A, which corresponds to 11 times the yield strain $\left(\varepsilon_{y}\right)$ and $55 \%$ greater than the maximum strain recorded for column $\mathrm{C} 1 \mathrm{C}$ L.

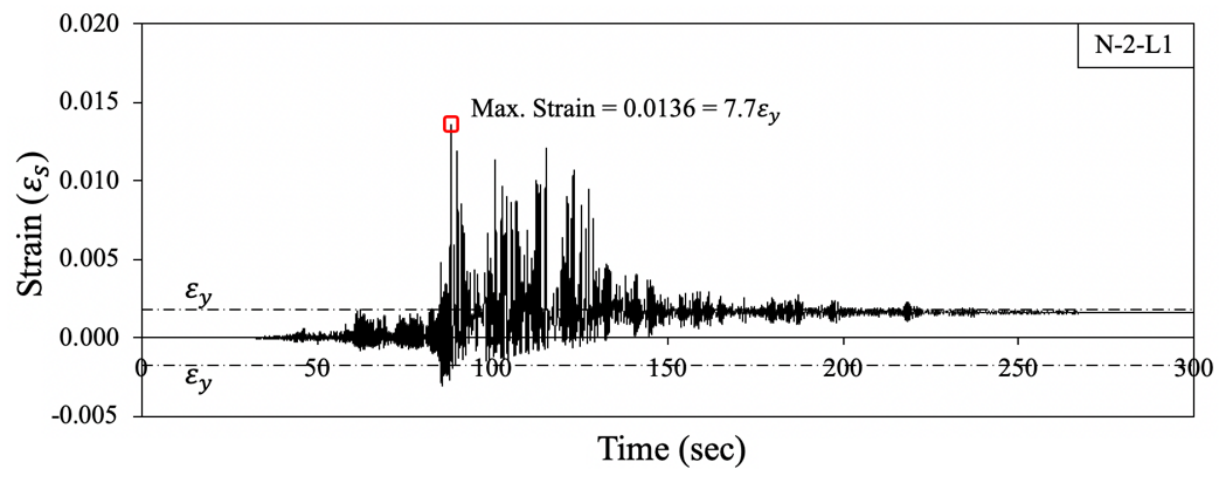

Figure 3.57 Steel strain history for strain gauge N-2-L1 for column C3C-T during motion amplitude $\mathrm{A}$

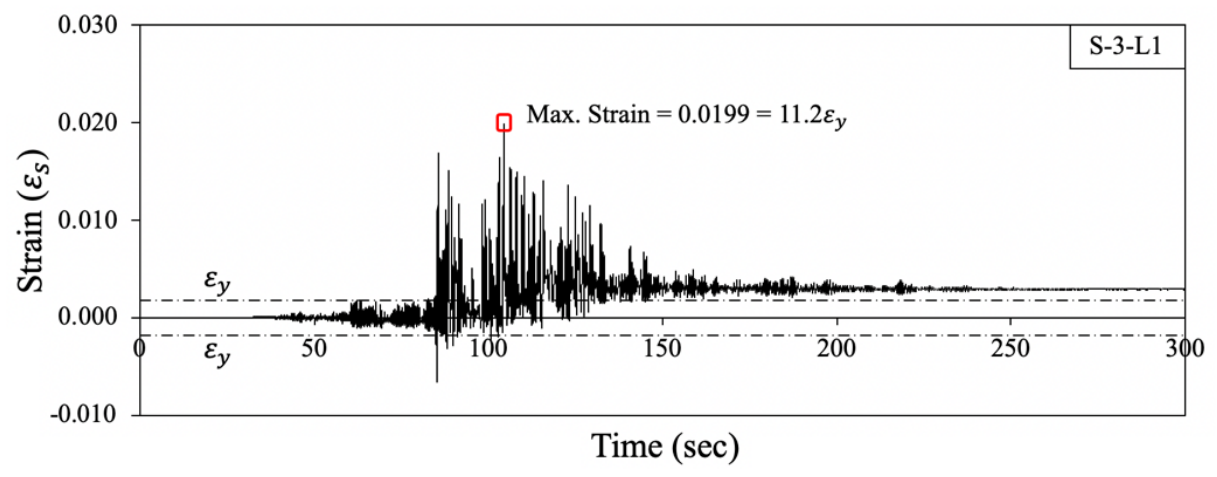

Figure 3.58 Steel strain history for strain gauge S-3-L1 for column C3C-T during motion amplitude A 


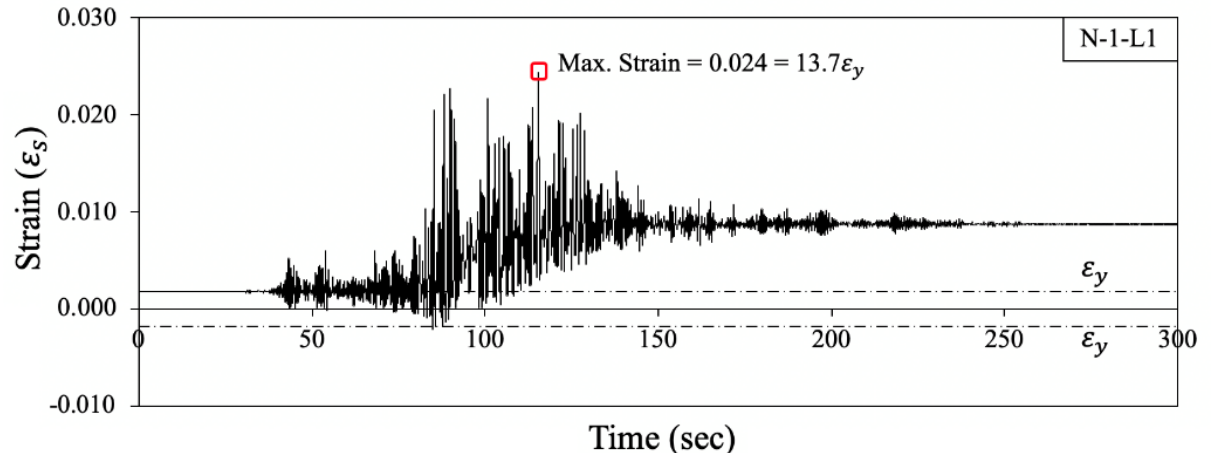

Figure 3.59 Steel strain history for strain gauge N-1-L1 for column C3C-T during motion amplitude B

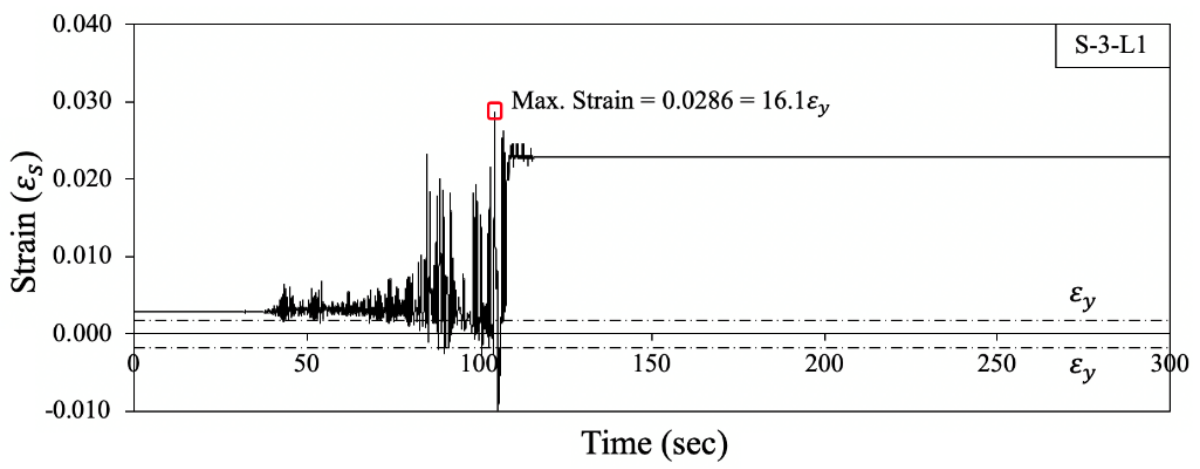

Figure 3.60 Steel strain history for strain gauge S-3-L1 for column C3C-T during motion amplitude B

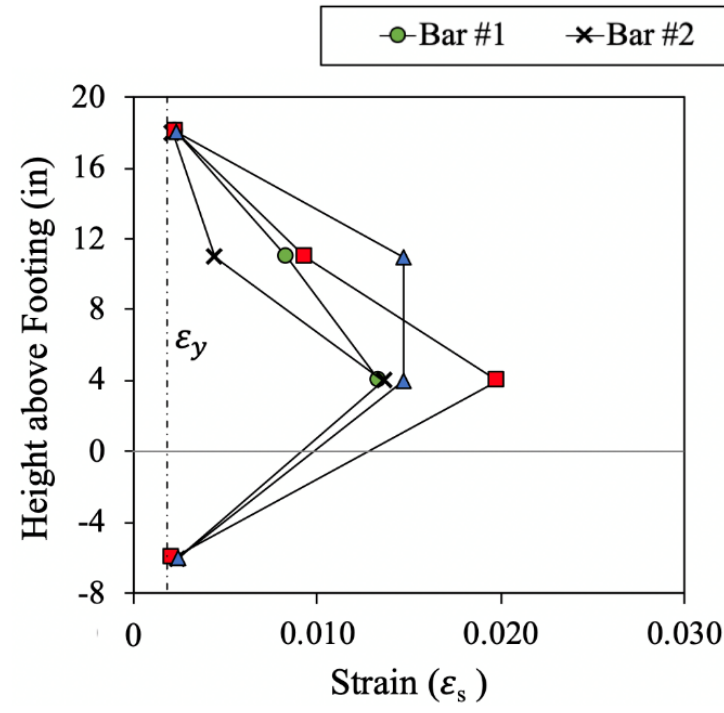

a)

\section{$\square-$ Bar \#3 $\triangle$-Bar \#4}

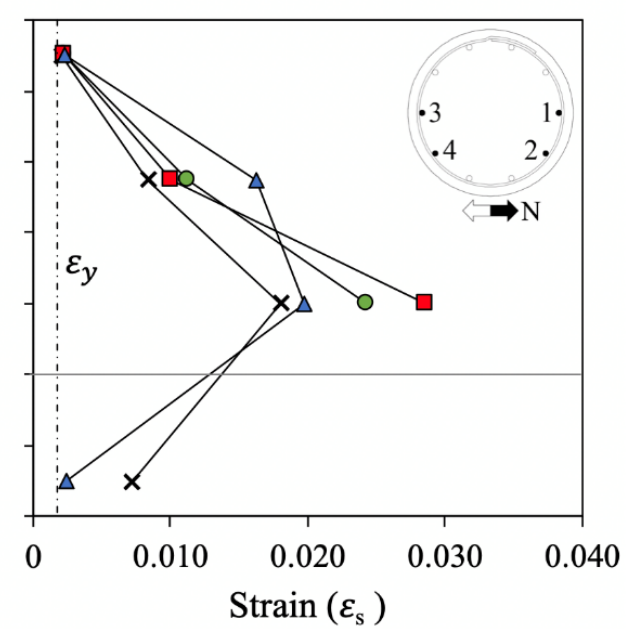

b)

Figure 3.61 Peak steel strain profile for column C3C-T. a) Amplitude A, b) Amplitude B 


\subsubsection{Curvature}

As mentioned before, linear variable displacement transducers (LVDTs) were used to measure the average curvature along the height of the column. The peak curvature profile along the height is shown in Figure 3.62 for each motion amplitude. The points in the curvature profiles represent the curvature measured at the specific location and in correspondence to the maximum and minimum peak values of column displacement. The curvature profiles in each direction were fairly symmetric, in agreement with the symmetry of the ground motion record. Also, as it was expected for columns tested on single curvature, large curvature values were measured at the base of the column, where the moments were larger and induced substantial nonlinear deformation. It is shown in the figure that the desired behavior was achieved in the plastic hinge region where the visible damage occurred (concrete cracks, spalling, core crushing, and bar buckling).

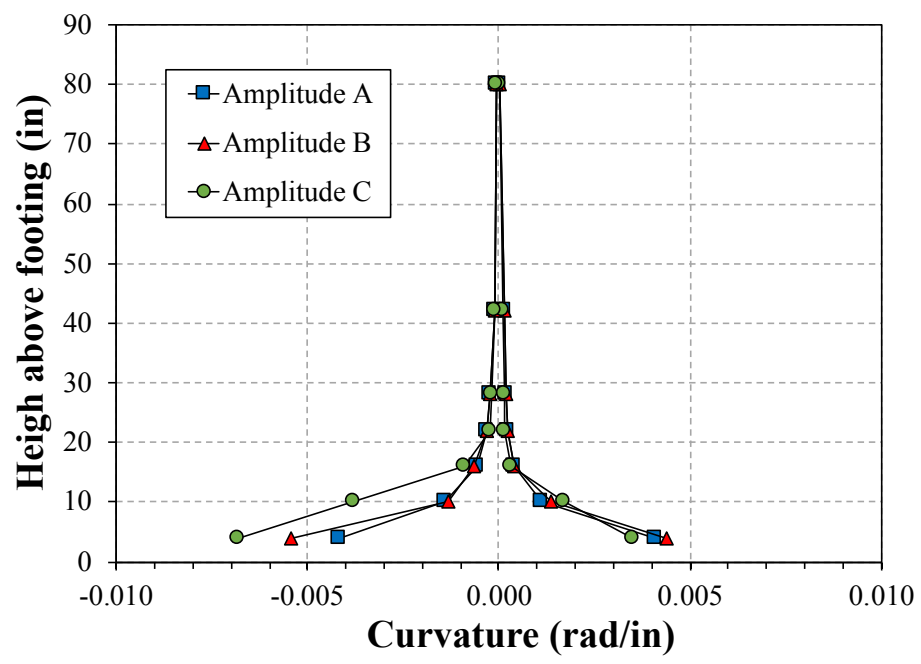

Figure 3.62 Average curvature profiles in column C3C-T 


\subsubsection{Axial Forces}

Although the experiments were designed to keep the same level of axial force during the test sequence, some variations were observed in each earthquake motion amplitude during testing. The axial force history for specimen C3C-T is shown in Figure 3.63. The target force was 75 kips $(334 \mathrm{kN})$ for motion amplitude A and B, and 60 kips (267 kN) for motion amplitude C. The maximum deviation from the target axial force was $-1.9 \%$ and $+24.1 \%$ in motion amplitude $\mathrm{A},-4.2 \%$ and $+23.5 \%$ in motion amplitude $\mathrm{B}$, and $-1.4 \%$ and $+26.8 \%$ in motion amplitude $\mathrm{C}$. The variations of the axial force may be attributed to the change in axial loads in the treaded rods as the specimen displaced. Normally, the accumulators compensate for this, but the hinging action of the load transfer beam created a less stiff axial load system. The less stiff system caused the hydraulic ram to displace further and faster than its ability to maintain constant axial load.

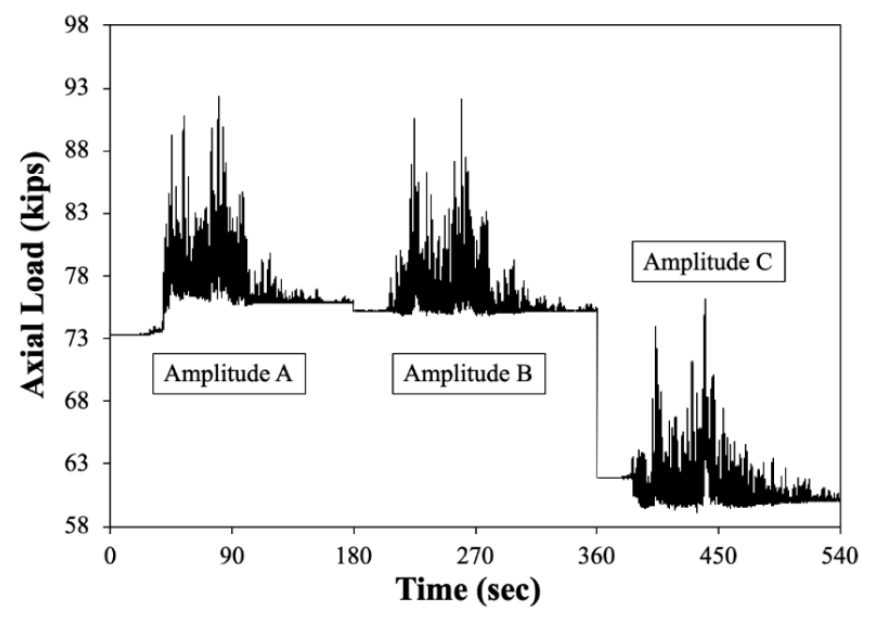

Figure 3.63 Axial force history for column C3C-T 


\subsubsection{Shake Table Output Motions}

Comparison between the input and output motions is also presented for the Iwaki Y record. For the input motion applied column C3C-T, small differences between the target and achieved accelerations reproduced by the shake table were observed despite the fine-tuning applied to the system. Comparison of the target and recorded time history accelerations for each motion amplitude are shown in Figure 3.64(a). Table 3.13 shows the target and achieved values of peak table acceleration (PGA), velocity (PGV), and displacement (PGD) for each motion amplitude. In addition to the peak responses comparison, 5\% damped elastic acceleration response spectrums were calculated from the target and achieved acceleration signals for each motion amplitude, as depicted in Figure 3.64(b). Also, it is shown in the same figures the measured initial and final period of the specimen. Although there are variations in the target and achieved elastic response accelerations for all the motions, these discrepancies are more important at the low and high period portion of the spectrum. The impact of these discrepancies is not significant since the potential specimen's response is in between that range of periods as it is shown in the spectrums. Therefore, the table performance is judged as satisfactory for the period range of interest. 


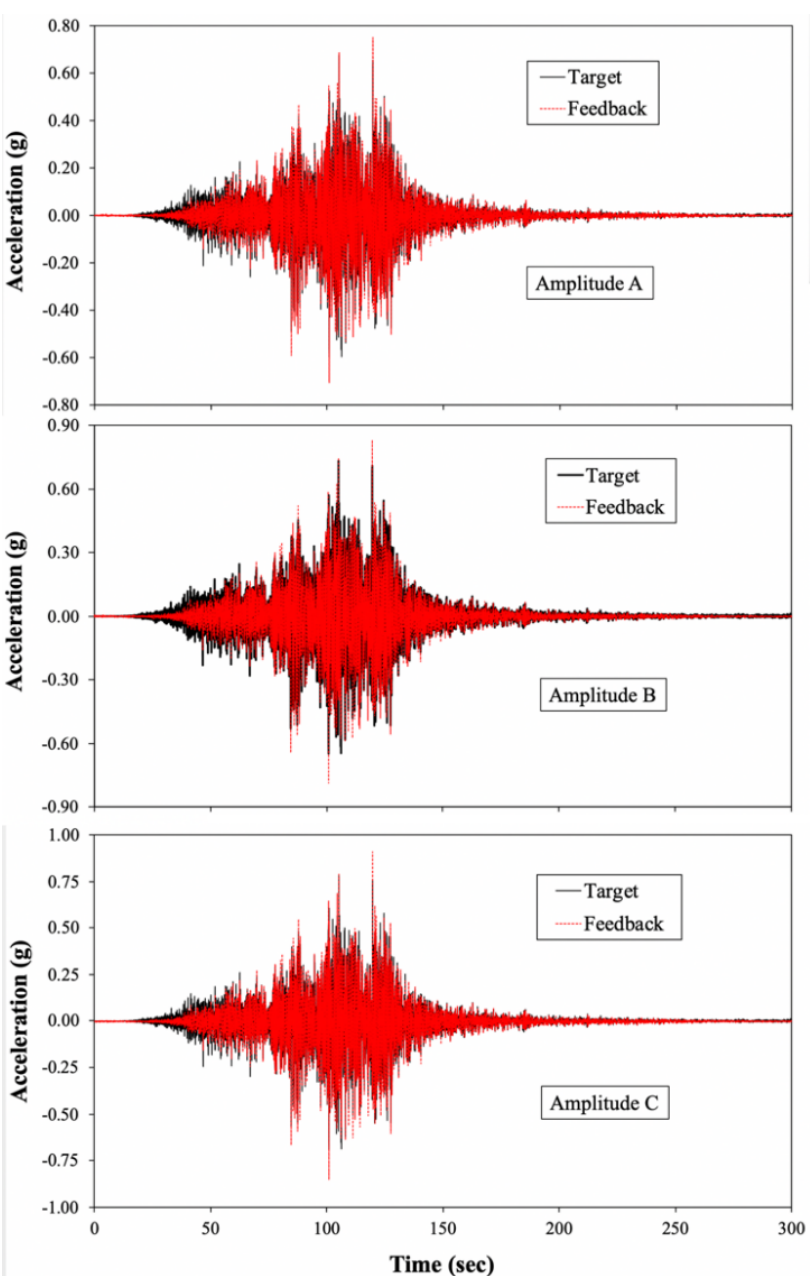

a)
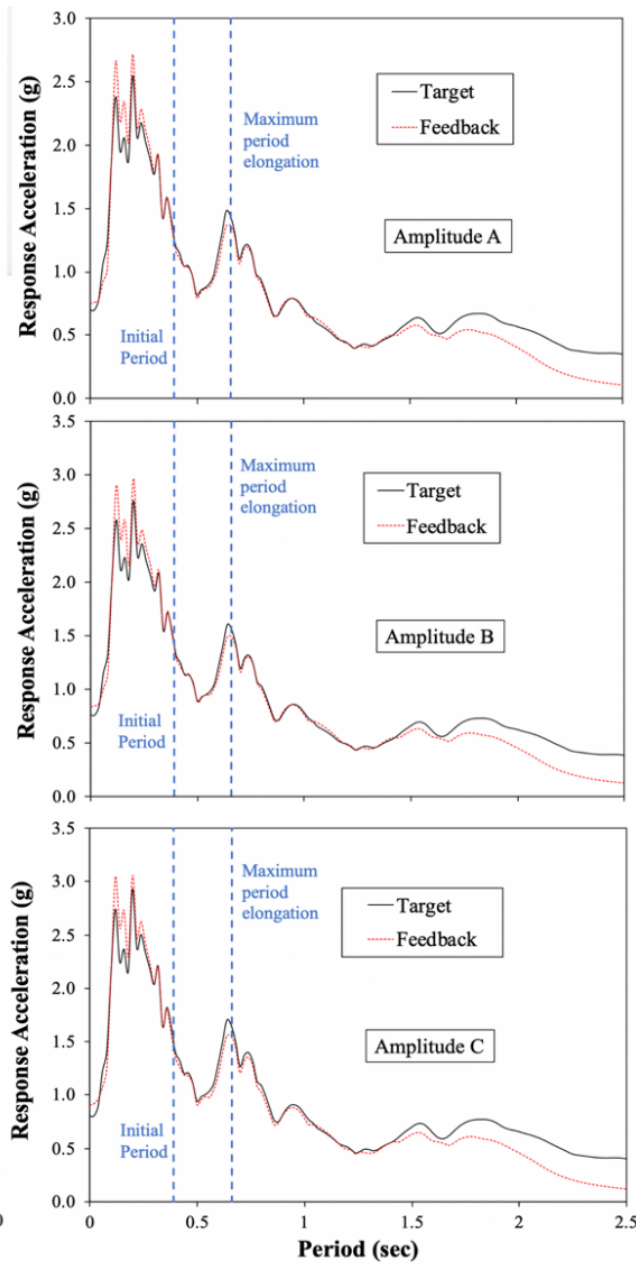

b)

Figure 3.64 Shake table performance for Iwaki Y. a) Acceleration time histories, b) 5\% damped elastic response spectra

Table 3.13 Target and achieved peak table responses for Iwaki Y

\begin{tabular}{c|c|c|c|c|c|c}
\hline \multirow{2}{*}{ Amplitude } & \multicolumn{2}{|c|}{ PGA $(\mathrm{g})$} & \multicolumn{2}{c|}{ PGV (in/sec) } & \multicolumn{2}{c}{ PGD (in) } \\
\cline { 2 - 7 } & Target & Achieved & Target & Achieved & Target & Achieved \\
\hline A & 0.67 & 0.75 & 17.71 & 21.00 & 4.26 & 3.39 \\
\hline B & 0.73 & 0.83 & 19.21 & 23.10 & 4.62 & 3.68 \\
\hline C & 0.78 & 0.91 & 20.41 & 24.14 & 4.91 & 3.84 \\
\hline
\end{tabular}




\subsubsection{Column C4S1-L}

Column C4S1-L was the fourth column tested. It was subjected to the Capitola X record from the 1989 Loma Prieta earthquake (crustal). In contrast to column C1C-L, this specimen was designed with a short lap splice at the expected hinge zone. Test results and discussion are presented in the following subsections.

\subsubsection{Progression of Observed Damage}

Before discussing the damage progression, it is worth mentioning that specimen C4S1-L was tested in a damaged condition due to high amplitude signal from the shake table system before running the correct motion. Damage was fully concentrated at the spliced region and characterized primarily by vertical cracks at the column base. Following first bar yielding, spalling of concrete cover occurred at the first earthquake motion amplitude. C4S1-L showed spalling of cover concrete at the base of the column on both sides and up to 4 in, but it was not significant compared to the Column C1C-L (and the other two specimens) mainly due to rocking at the cold joint in the columnfooting interface. Vertical cracks consistent with lap splice failure on the north and south side were also noticed, as shown in Figure 3.65. Motion amplitude B caused the complete collapse of the column, as shown in Figure 3.66. During testing, the splitting cracks along the spliced length suddenly opened due to the slippage of the spliced bars. The deterioration of the lap splice bond was immediately followed by concrete cover spalling and concrete crushing. Additionally, horizontal and vertical cracks propagated over the splice zone around the circumference of the column. Extensive damage in the column core was also observed, which reduced the axial capacity of the column. After 
completion of testing, all concrete cover along the entire splice length $\left(20 d_{b}\right)$ had spalled off.

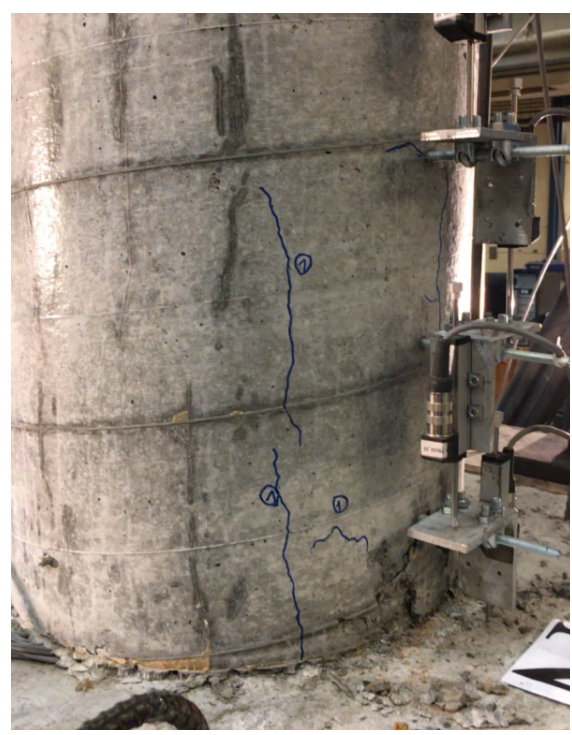

a)

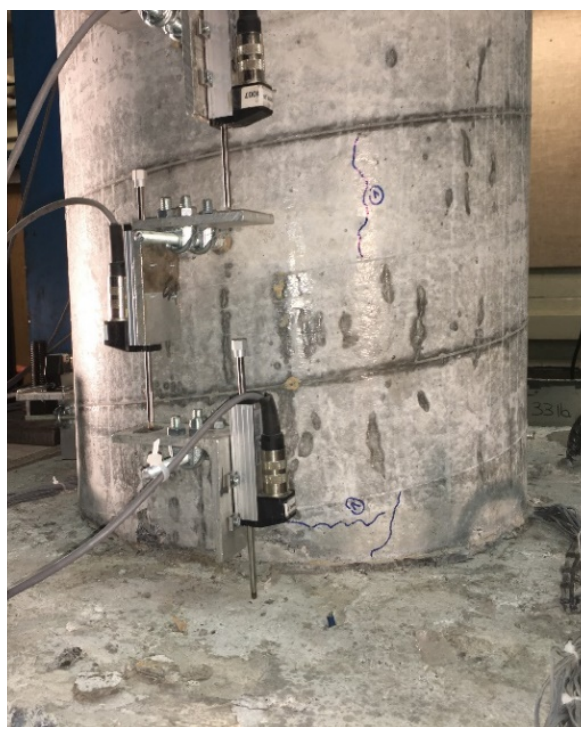

b)

Figure 3.65 Damage state of column C4S1-L after applying motion amplitude A. a) North-east view, b) south-east view

As expected for a cantilever column, the damage was fully localized to the lower part of the column in the plastic hinge region. Figure 3.65 and Figure 3.66 show the different visual damage states for Column C4S1-L after each ground motion amplitude. The primary mode of failure was lap splice failure followed by crushing of concrete core. Figure 3.67 shows the specimen at completion of testing. 


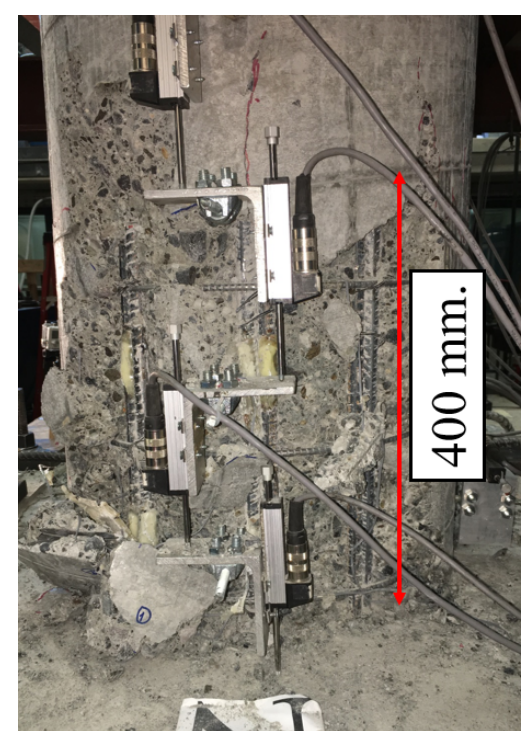

a)

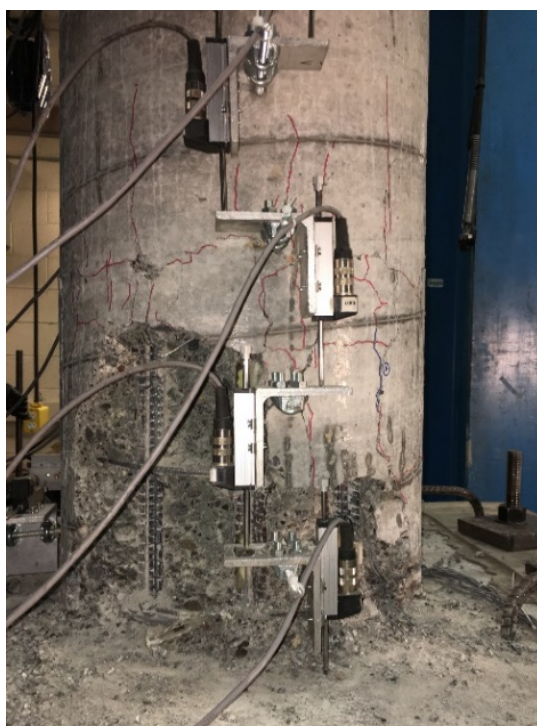

b)

Figure 3.66 Damage state of column C4S1-L after applying motion amplitude B. a) North view, b) south-west view

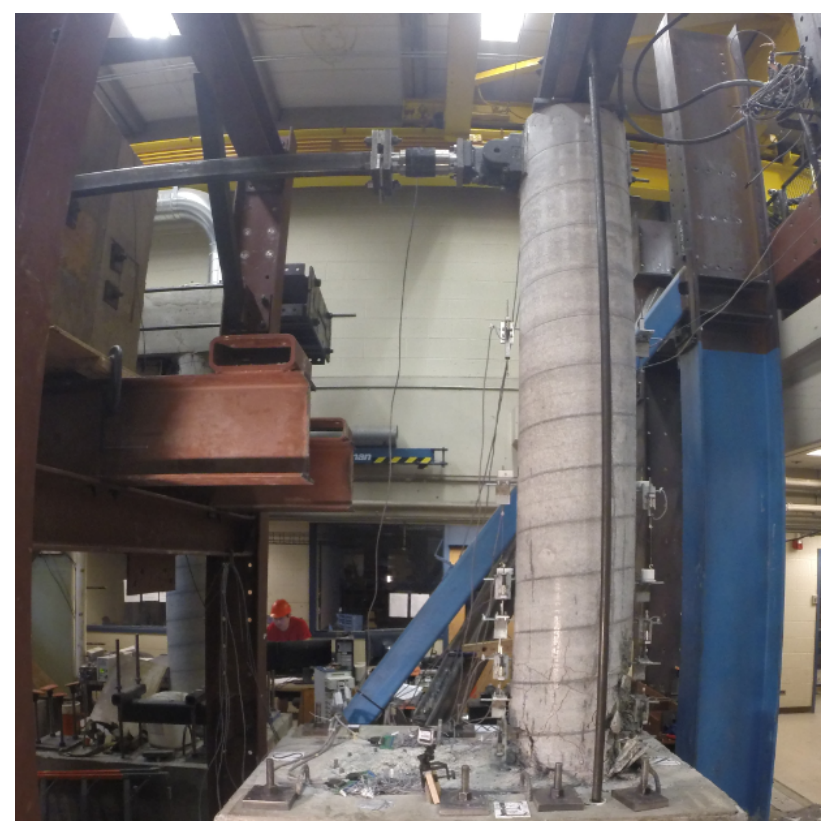

Figure 3.67 Final state of column C4S1-L

\subsubsection{Force-Displacement Relationship}

The measured hysteresis curves for column C4S1-L for all ground motion amplitudes are shown in Figure 3.68 and Figure 3.69. Damage states are also identified 
on the force-displacement response histories. As was mentioned previously, the specimen was loaded in the north-south direction, which means that positive displacement direction corresponds to the north direction, and negative displacement direction is towards the south direction.

For motion amplitude A, column C4S1-L showed a low level of ductility by reaching a displacement ductility of $\mu=2.3$ ( $\Delta=2.9$ in) on both directions (Figure 3.68). No significant reduction of lateral strength for this column was observed after the first motion amplitude; thus C4S1-L was able to sustain another amplitude increment. After a few cycles in motion amplitude B, lateral strength of C4S1-L degraded abruptly due to bond deterioration along the splice. This degradation is indicated in the hysteresis curve by the green dashed line (Figure 3.69). Results of column C4S1-L revealed the difference in column performance when varying the splice length. Furthermore, the response of column C4S1-L was characterized as non-ductile and limited energy dissipation capacity.

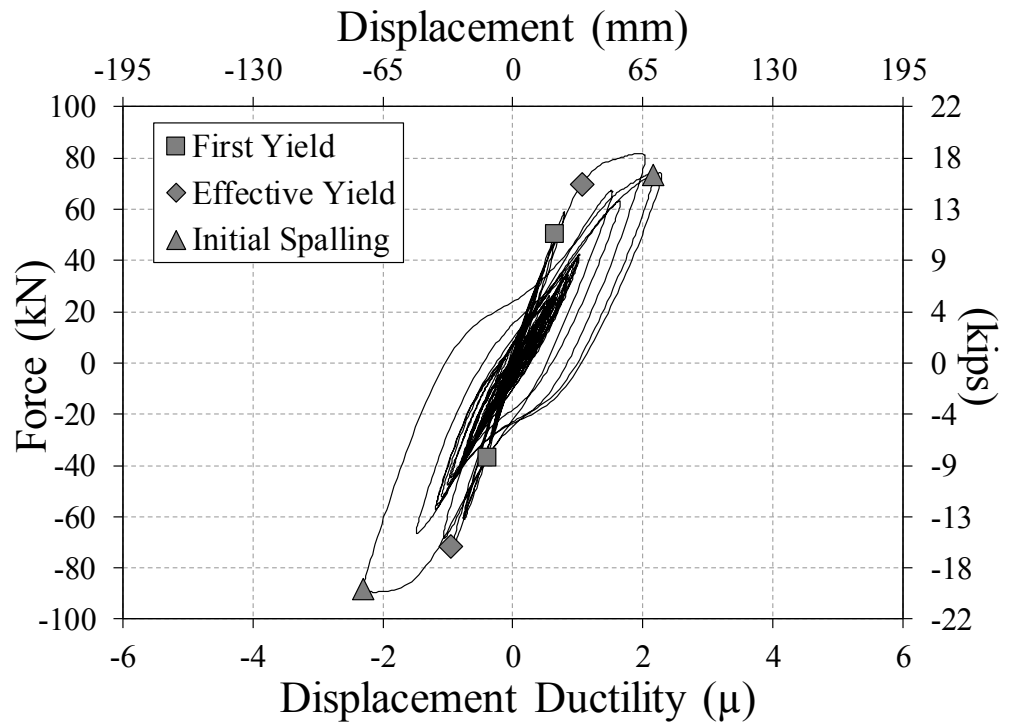

Figure 3.68 Hysteretic response for column C4S1-L during motion amplitude A 


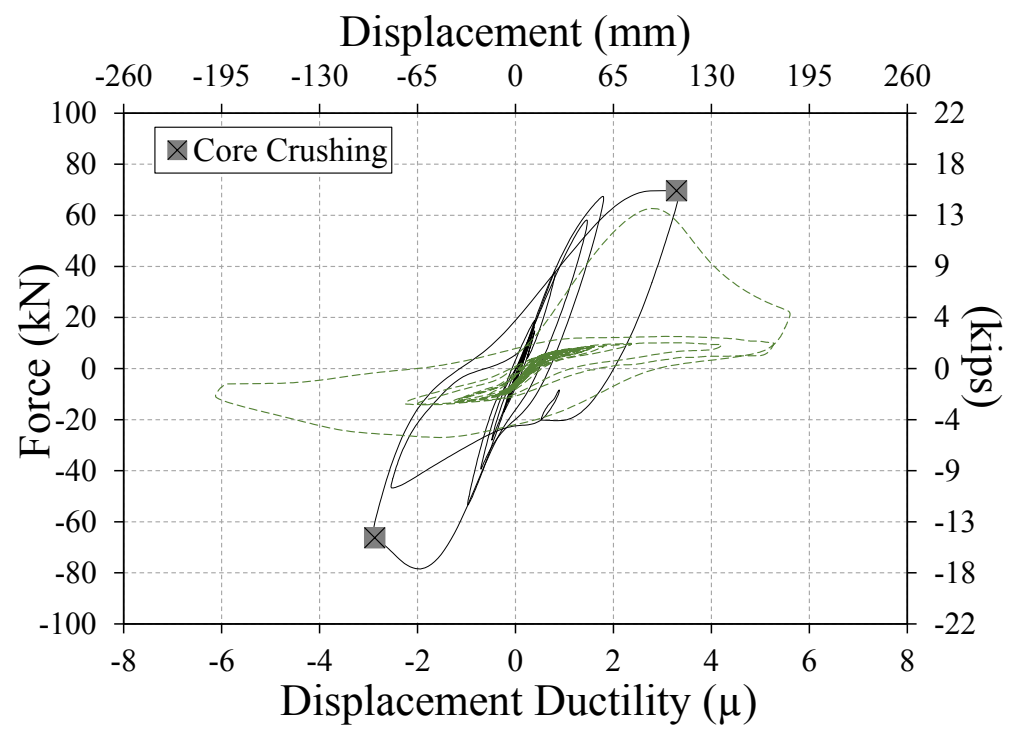

Figure 3.69 Hysteretic response for column C4S1-L during motion amplitude B

The maximum displacement for each earthquake motion amplitude, and its corresponding drift ratio and ductility level are presented in Table 3.14. These values are the peak displacement values and do not correspond to the peak lateral force values, which are also listed in Table 3.5. Reported displacement ductility levels are based on the effective yield displacement, which was found to be $1.28 \mathrm{in}$. This value was computed using the reduce stiffness method assuming an elasto-plastic idealization. Even though C4S1-L was detailed with a short lap splice, strain gauges placed on the extreme starter bars reached the yield strain. Given that the specimens were already damaged, it allowed the spliced bars to engage and transfer the forces to generate yield. The displacement history for column C4S1-L is shown in Figure 3.70. It also indicates the moment at which the lap-spice failure occurred (green dashed line). On the other hand, it is seen in Figure 3.70 and in Table 3.14 that column C4S1-L experienced little residual displacement for the first motion amplitude. 


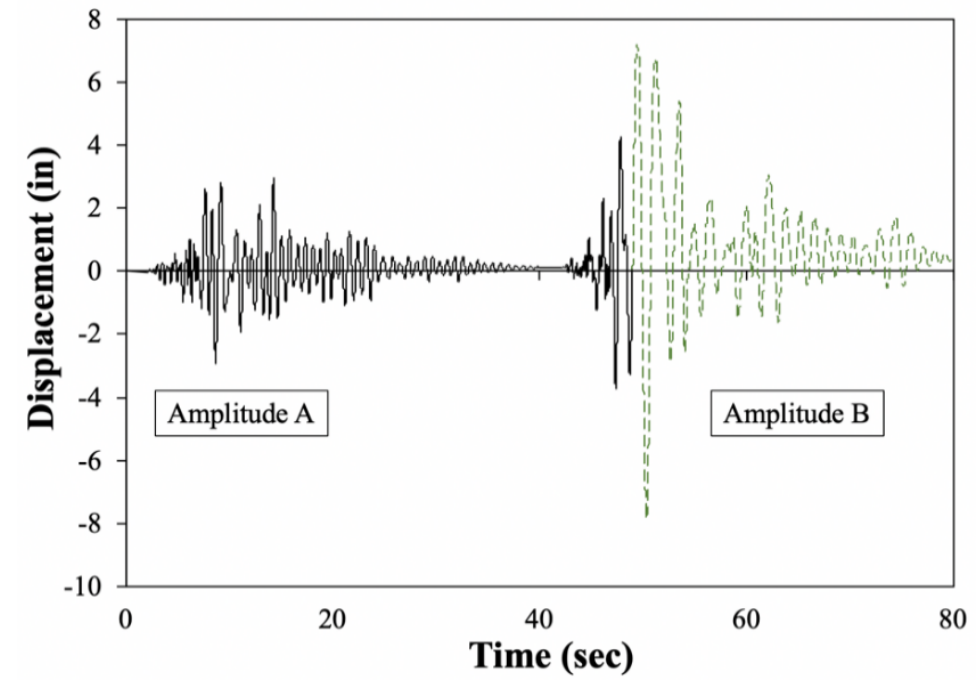

Figure 3.70 Column displacement history for column C4S1-L

Table 3.14 Summary of the measured performance for C4S1-L

\begin{tabular}{c|c|c}
\hline Property & Amplitude A & Amplitude B \\
\hline $\begin{array}{c}\text { Max. Displacement } \\
\text { in. }(\mathrm{mm})\end{array}$ & $2.9(74.6)$ & $7.8(199.1)$ \\
\hline $\begin{array}{c}\text { Max. Drift Ratio } \\
(\%)\end{array}$ & 3.06 & 8.17 \\
\hline $\begin{array}{c}\text { Max. Displacement } \\
\text { Ductility Level }(\mu)\end{array}$ & 2.3 & 6.1 \\
\hline $\begin{array}{c}\text { Residual } \\
\text { Displacement in. } \\
(\mathrm{mm})\end{array}$ & $0.13(3.3)$ & $0.42(10.7)$ \\
\hline $\begin{array}{c}\text { Max. Base Shear } \\
\text { kips }(\mathrm{kN})\end{array}$ & $20.1(89.4)$ & $17.6(78.5)$ \\
\hline
\end{tabular}

\subsubsection{Dynamic Properties}

Low-level white noise signals, as well as pulse tests, were performed before and in between earthquake motion amplitudes to monitor the change in the dynamic properties due to damage progression in the specimen. Natural periods were determined from Fourier analysis of the accelerations measured at the top of the column. The damping ratio was calculated from the free vibration portion of the accelerograms 
recorded at the top of the specimen using the decrement logarithmic method. Since the free-vibration portion of the accelerogram does not have a perfect exponential decay, the accelerogram was divided into various intervals for which a damping ratio was calculated. Then, a mean value was calculated for the damping ratio. A summary of the computed periods and mean damping ratio values and standard deviations are presented in Table 3.15. The uncracked natural period of the column model was $0.33 \mathrm{sec}$; however, it is not included in Table 3.15, because specimen C4S1-L was tested in a damaged condition due to high amplitude signal from the shake table system before running the correct motion. Therefore, the initial period reported in Table 3.15 represents the cracked natural period. From the results, it is clear that the computed natural periods were lengthened with the increased level of excitation, what confirmed the deterioration of column stiffness. The variation in damping followed the same trend, i.e., it increased with the progression of the damage from $3.2 \%$ to $5.5 \%$. In general, good agreement was observed between the results of both tests.

Table 3.15 Measured dynamic properties for C4S1-L

\begin{tabular}{c|c|c|c|c}
\hline \multirow{2}{*}{ Sequence } & \multicolumn{2}{|c|}{ Period, $T_{n}(\mathrm{sec})$} & \multicolumn{2}{c}{ Damping ratio, $\zeta(\%)$} \\
\cline { 2 - 5 } & Pulse test & W-N random test & $\mu_{X}$ & $\sigma_{X}$ \\
\hline Initial & 0.43 & 0.44 & 3.2 & 0.37 \\
\hline amplitude A & 0.59 & 0.60 & 4.2 & 0.92 \\
\hline amplitude B & 0.69 & 0.69 & 5.5 & 0.59 \\
\hline
\end{tabular}

\subsubsection{Strains}

Reinforcing steel strains were recorded in the plastic hinge region of the specimen using strain gauges. Figure 3.71 through Figure 3.74 show the recorded steel strain 
histories in two of the dowels during motion amplitudes A and B for the tested column. The designation used in the figures is as follows: column position (N: north or S: south), the dowel bar number (D1, D2, D3 or D4), and the location (level) along the height (F: footing, L1: level 1, L2: level 2, and L3: level 3). For example, N-D1-L2 corresponds to North - dowel bar \#2 - Level 2. The used strain gauges were able to record most of the strain measurements, though some data is missing because they malfunctioned, most likely due to damage during construction or during testing. There was some initial strain in the reinforcement prior to shake table testing due to the applied axial loading. For Column C4S1-L, three strain gauges were affixed to the column longitudinal bars and to the starter bars anchored into the footing. Also, it is known that longitudinal strain is equal to zero at the ends of the longitudinal reinforcement bars. Based on the measured and known values, peak steel strain profiles were derived and plotted (Figure 3.75) for Specimen C4S1-L along the splice length. Lateral strength degradation coincided with the initiation of bond deterioration along the splice length, and bond deterioration was most significant at the bottom of the splice length where the maximum moment occurred. After the bond deteriorated there was no mechanism for transferring the strains between the starter bars and the column bars. A maximum strain of 0.029 in/in was measured in the north dowel bar during motion amplitude A, which corresponds to 16.3 times the yield strain $\left(\varepsilon_{y}\right)$. 


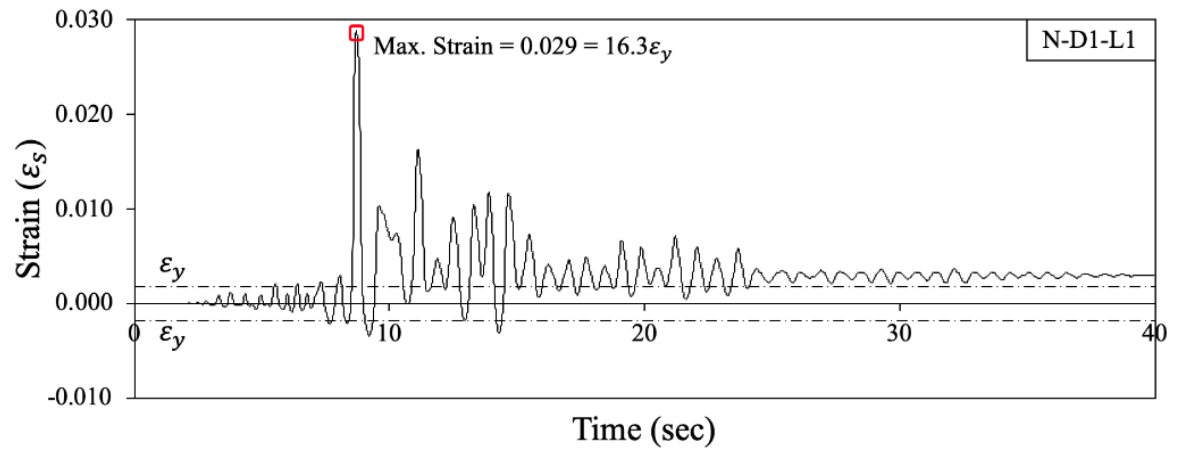

Figure 3.71 Steel strain history for strain gauge N-D1-L1 for column C4S1-L during motion amplitude $\mathrm{A}$

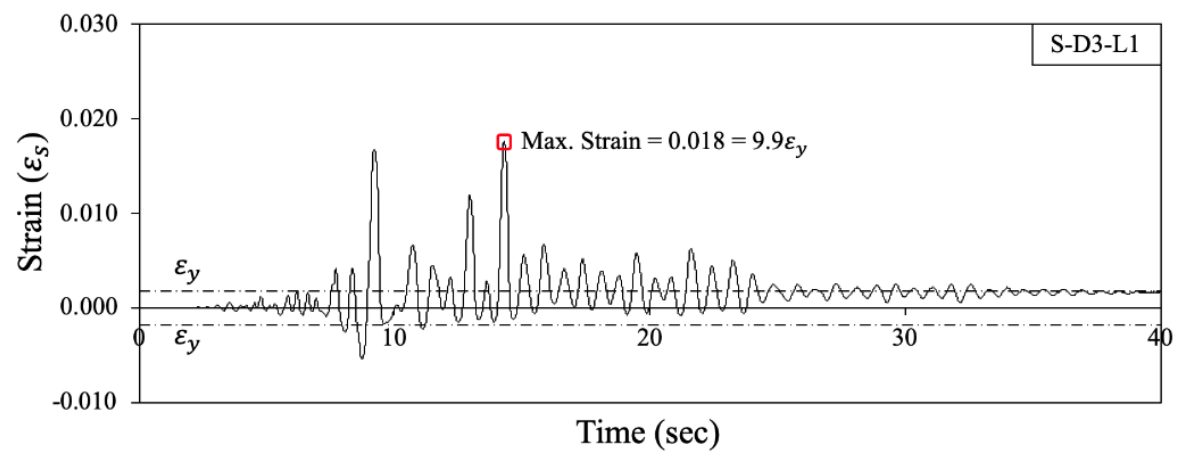

Figure 3.72 Steel strain history for strain gauge S-D3-L1 for column C4S1-L during motion amplitude $\mathrm{A}$

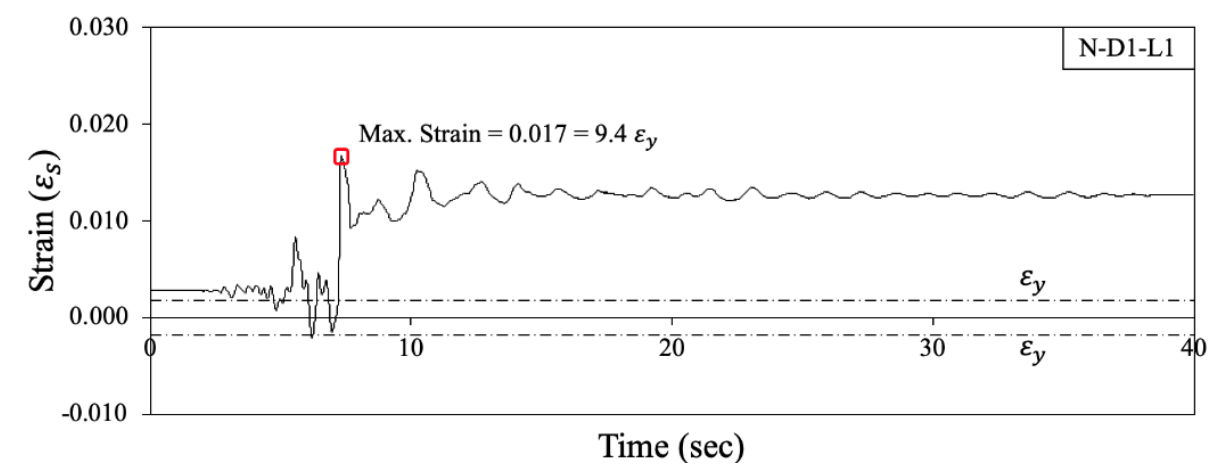

Figure 3.73 Steel strain history for strain gauge N-D1-L1 for column C4S1-L during motion amplitude B 


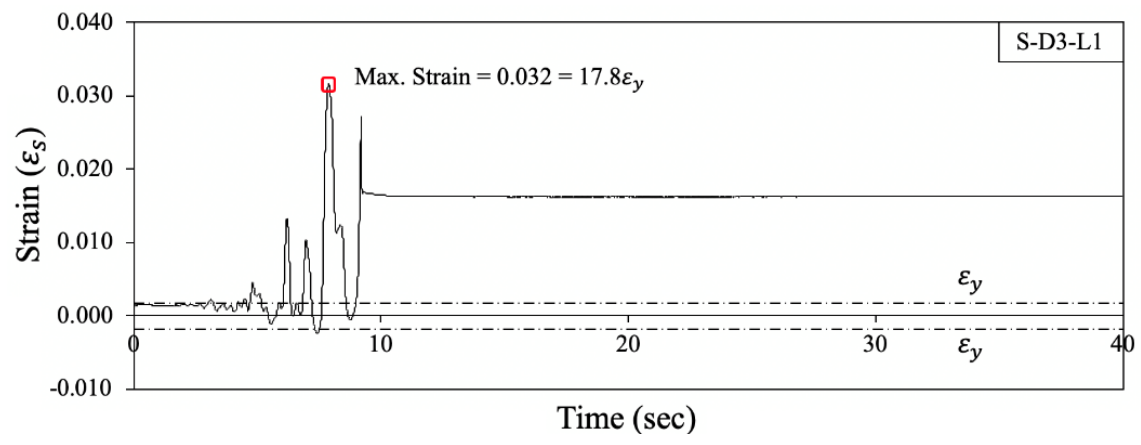

Figure 3.74 Steel strain history for strain gauge S-D3-L1 for column C4S1-L during motion amplitude B

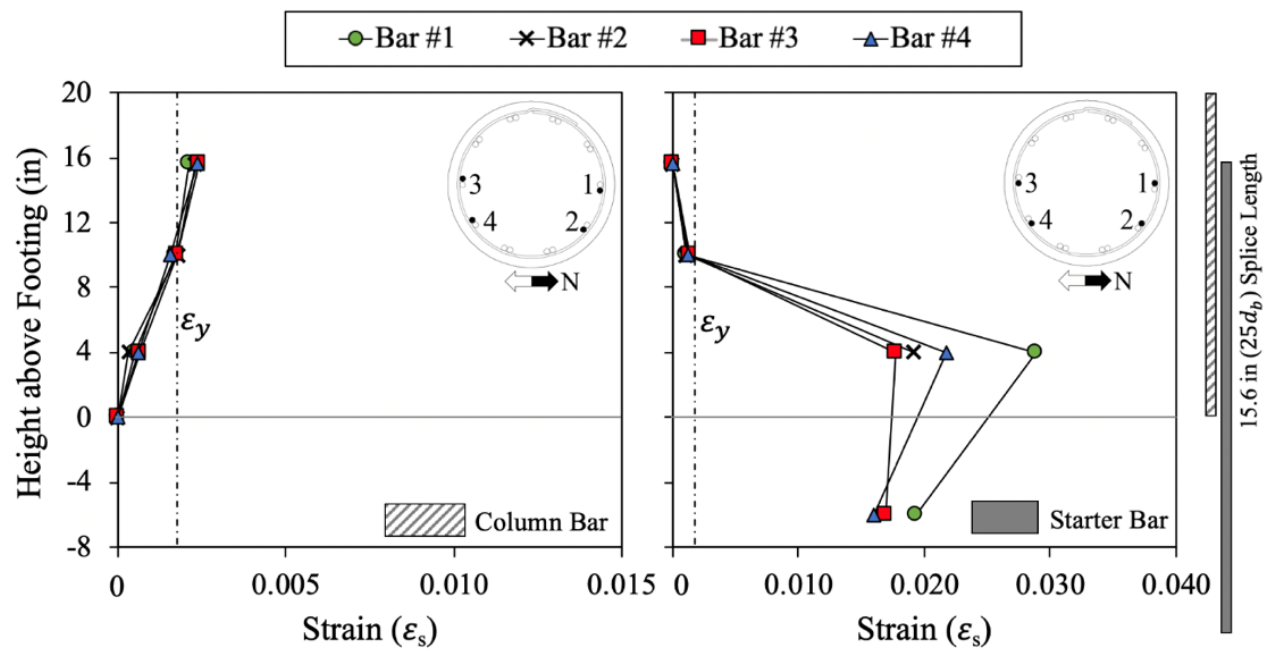

a)
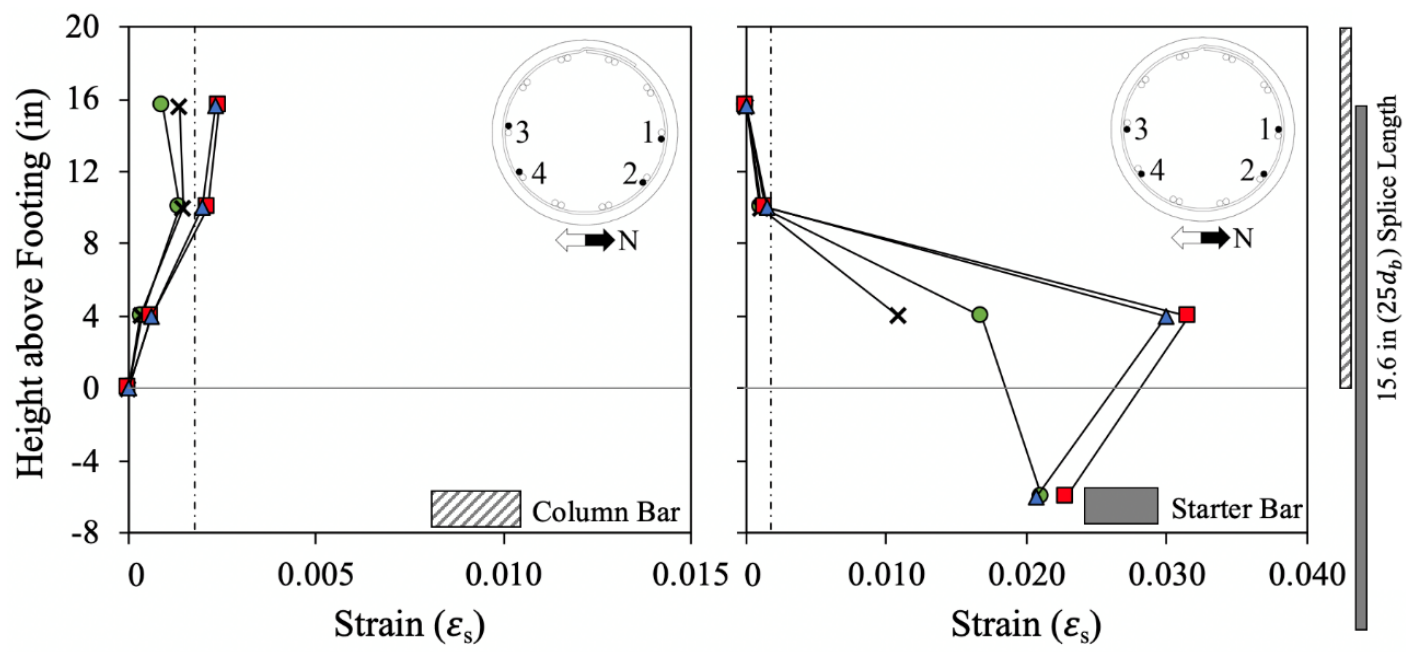

b)

Figure 3.75 Peak steel strain profile for column C4S1-L. a) Amplitude A, b) Amplitude B 


\subsubsection{Curvature}

As mentioned before, linear variable displacement transducers (LVDTs) were used to measure the average curvature along the height of the column. The peak curvature profile along the height is shown in Figure 3.76 for each motion amplitude. The points in the curvature profiles represent the curvature measured at the specific location and in correspondence to the maximum and minimum peak values of column displacement. During motion amplitude A, the low average curvatures in sections above the interface and within the splice agree with the minor damage observed in the column. For motion amplitude B, column curvatures were distributed along the spliced zone in the hinge where extensive damage was observed. However, it is seen in the figure that the average curvatures calculated at the column-footing interface were orders of magnitude higher because they include contributions from curvature and bond slip. Thus, the relative magnitudes demonstrate that the deformations from bond slip control the response at the interface, emphasizing the visual observations of bond-slip failures. These measurements further validated the visual observations of bond slip failure along the splice region of the column during the second motion. 


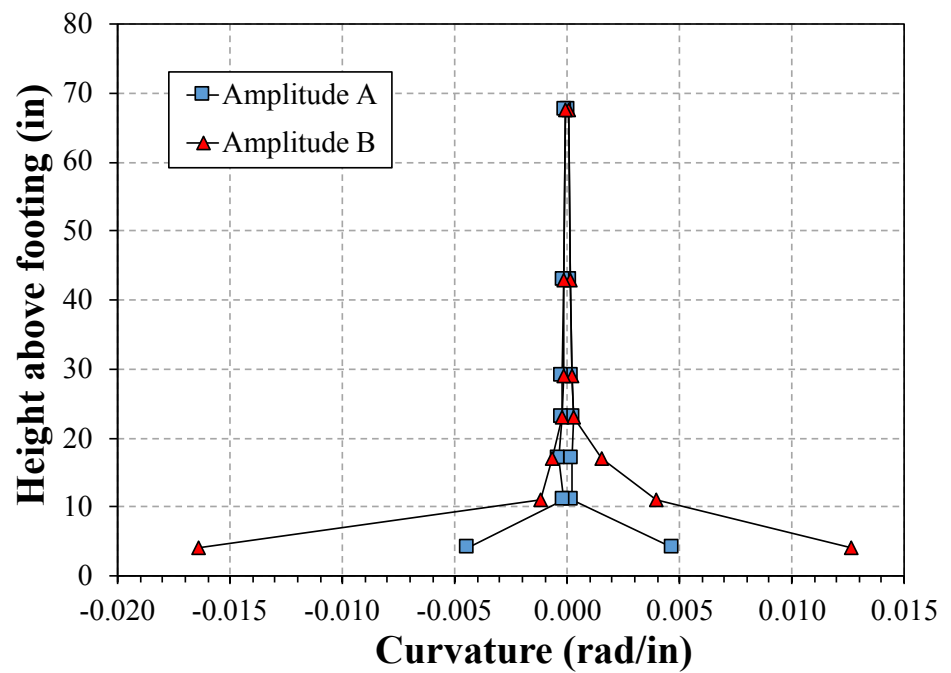

Figure 3.76 Average curvature profiles in column C4S1-L

\subsubsection{Axial Forces}

Although the experiments were designed to keep the same level of axial force during the test sequence, some variations were observed in each earthquake motion amplitude during testing. The axial force history for specimen C4S1-L is shown in Figure 3.77. The target force was 75 kips $(334 \mathrm{kN})$ for motion amplitude $\mathrm{A}$ and $\mathrm{B}$. The maximum deviation from the target axial force was $-16.6 \%$ and $+24.7 \%$ in motion amplitude $\mathrm{A}$, and $-44.3 \%$ and $+61.3 \%$ in motion amplitude $\mathrm{B}$. The variations of the axial force may be attributed to the change in axial loads in the treaded rods as the specimen displaced. Normally, the accumulators compensate for this, but the hinging action of the load transfer beam created a less stiff axial load system. The less stiff system caused the hydraulic ram to displace further and faster than its ability to maintain constant axial load. However, the high deviation from the target axial during motion amplitude B was due to the crushing of concrete core following the lap-splice failure, which led to the loss of axial capacity and relaxation of the post-tensioning rods. 


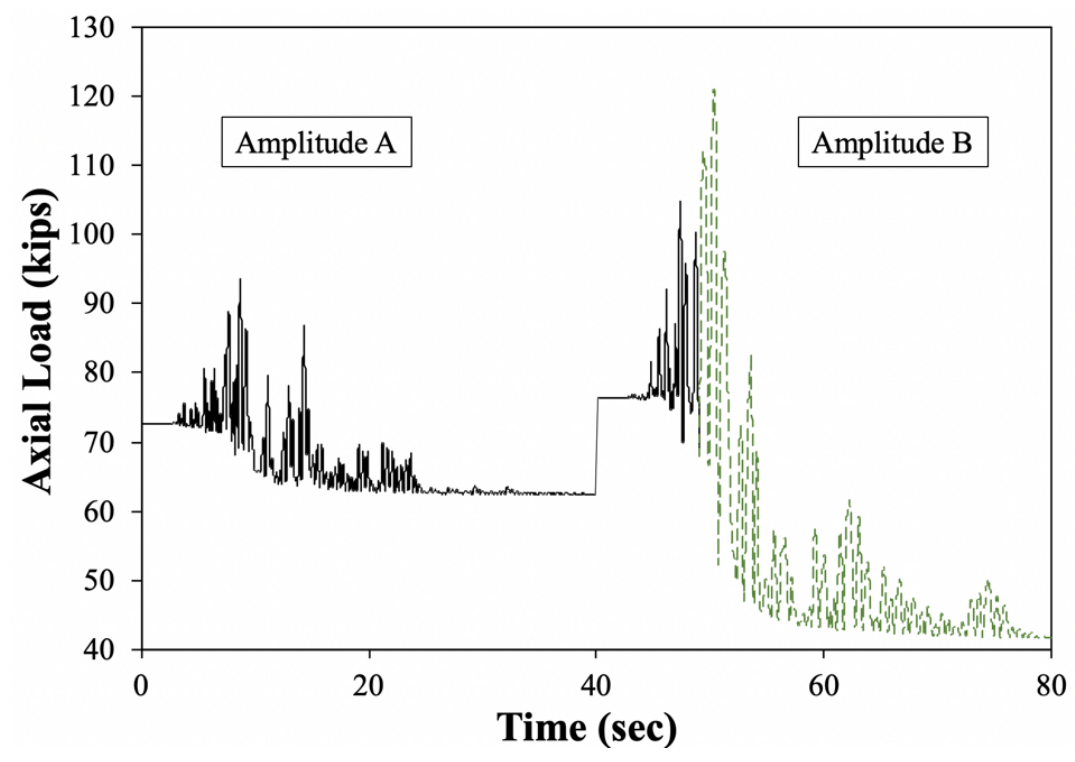

Figure 3.77 Axial force history for column C4S1-L

\subsubsection{Column C5S1-M}

Column C5S1-M was the fifth column tested. It was subjected to the Curico X record from the 2010 Maule earthquake (subduction). As in column C4S1-L, this specimen was designed with a short lap splice at the hinge zone with the aim of contrast the damage differences between reinforcement detailing. Test results and discussion are presented in the following subsections.

\subsubsection{Progression of Observed Damage}

As it happened with column C4S1-L, specimen C5S1-M was tested in a damaged condition due to high amplitude signal from the shake table system before running the correct motion. Column C5S1-M was only subjected to motion amplitude A and caused its complete failure. Considerable damage was observed in C5S1-M and characterized by horizontal and vertical cracks that propagated over the spliced length all around the circumference of the column, as shown in Figure 3.78(a). The vertical cracks were well 
defined due to slippage of the longitudinal bars and gave place to extensive crushing of core concrete at the base of the column on the north and south side, as depicted in Figure 3.78(b). This phenomenon was followed by extensive damage in the column core which reduced the axial capacity of the column. There was also visible rocking at the cold joint between the column and the footing. This specimen suffered more damage than $\mathrm{C} 2 \mathrm{C}-\mathrm{M}$ for the same motion amplitude. Interestingly, C4S1-L and C5S1-M showed the same severe lap-splice failure, but for a different motion amplitude, i.e., C4S1-L failed at motion amplitude B whereas C5S1-M failed at motion amplitude A. This outcome will be further discussed in a following section.

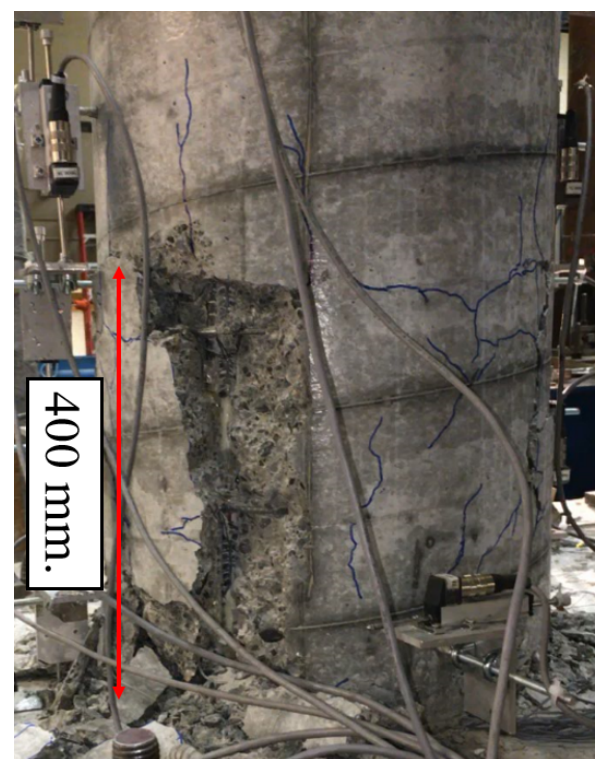

a)

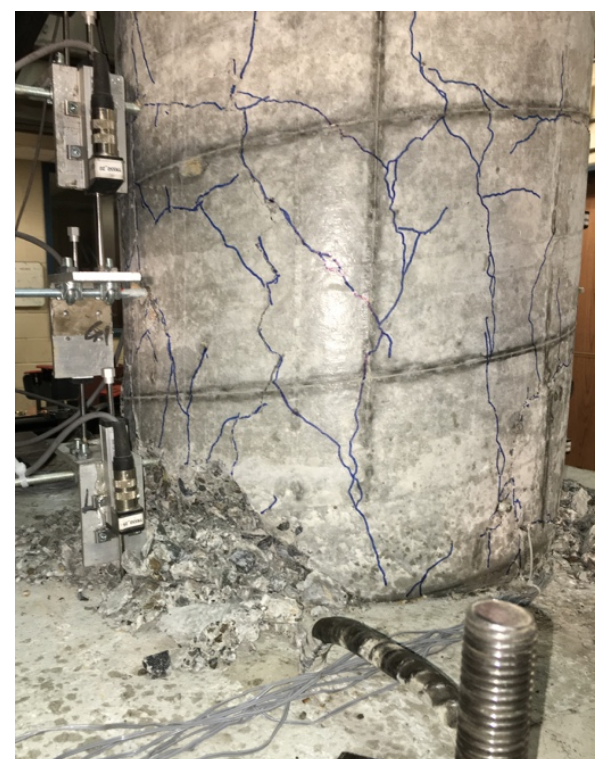

b)

Figure 3.78 Damage state of column C5S1-M after applying motion amplitude A. a) North-west view, b) south-east view 


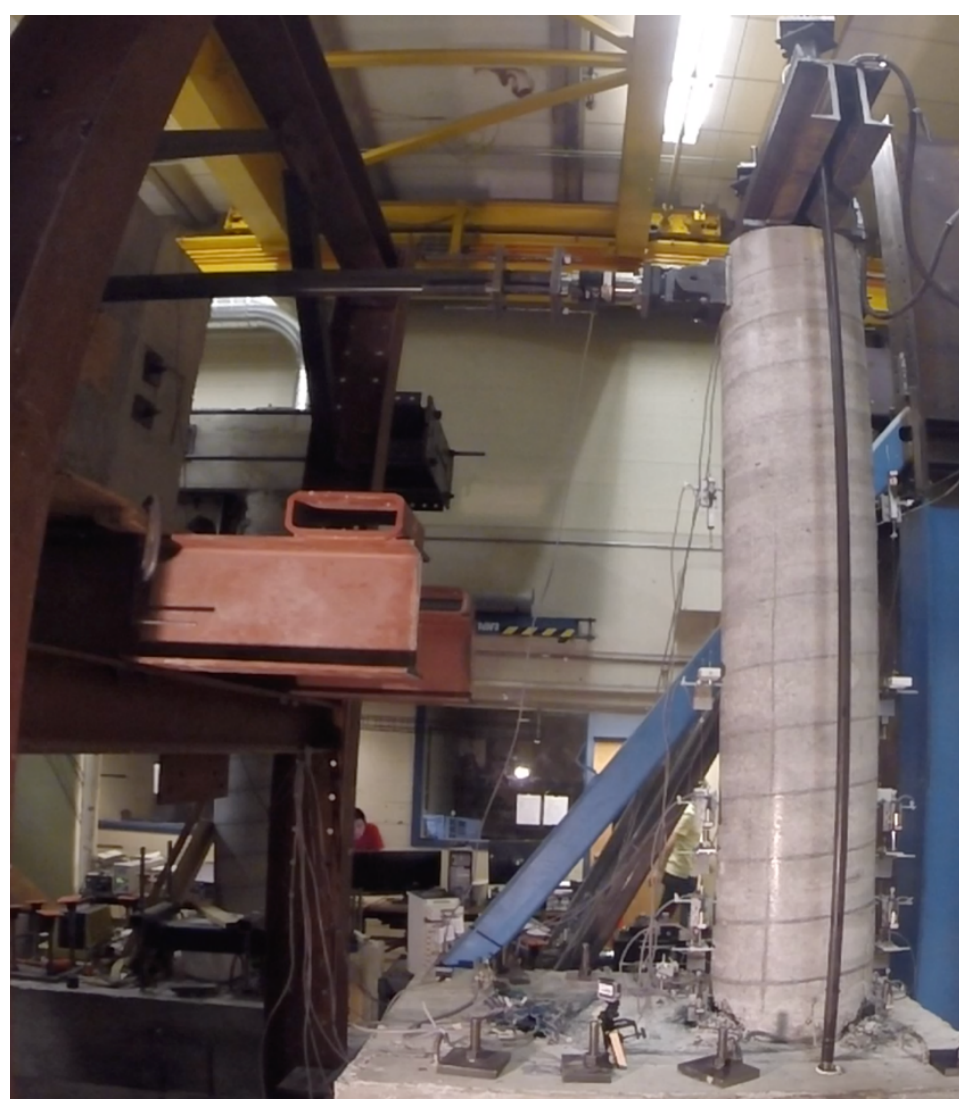

Figure 3.79 Final state of column C5S1-M

As expected for a cantilever column, the damage was fully localized to the lower part of the column in the plastic hinge region. Figure 3.79 shows the specimen at completion of testing.

\subsubsection{Force-Displacement Relationship}

The measured hysteresis curve for column C5S1-M is shown in Figure 3.80. Damage states are also identified on the force-displacement response history. As was mentioned previously, the specimen was loaded in the north-south direction. This means that positive displacement direction corresponds to the north direction, and negative displacement direction is towards the south direction. 


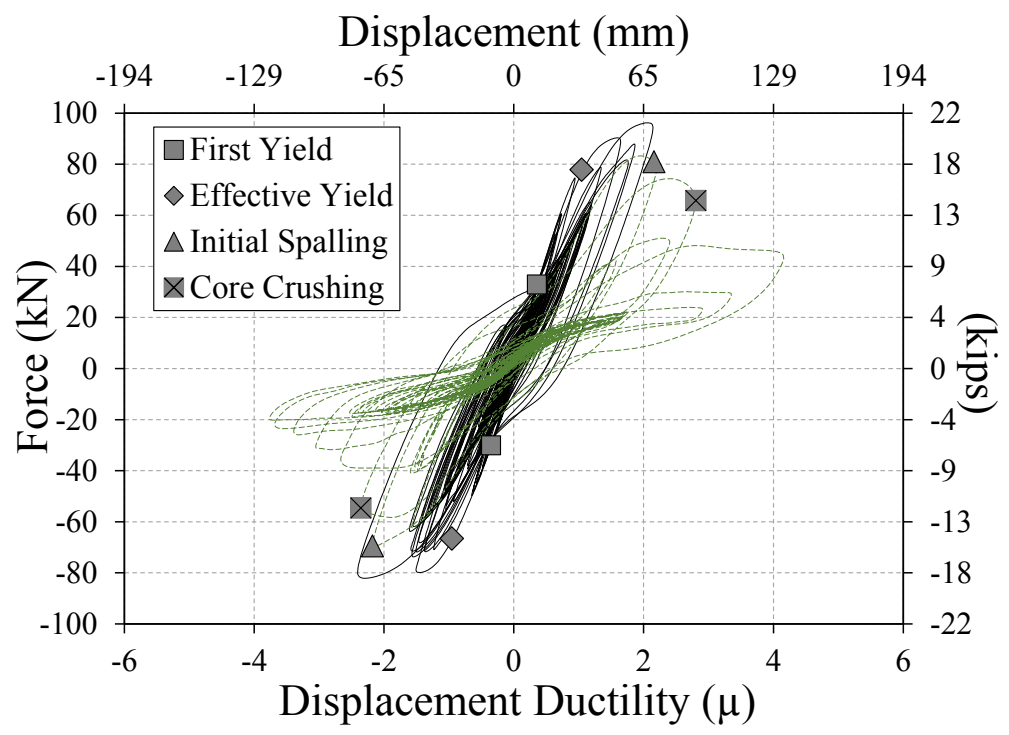

Figure 3.80 Hysteretic response for column C5S1-M during motion amplitude A C5S1-M showed a response similar to that of specimen C4S1-L before reaching the peak lateral strength capacity during motion amplitude A. However, C5S1-M exhibited sudden lateral strength degradation just after reaching its peak capacity. This sudden lateral strength degradation was due to slippage of the spliced bars and crushing of core concrete, and is indicated in the hysteresis curve by the green dashed line. The primary mode of failure for C5S1-M was lap splice failure in amplitude A. Results of column C5S1-M revealed the difference in column performance when varying the splice length and its location and the duration of the earthquake record. Furthermore, the response of column C5S1-M was characterized as non-ductile and limited energy dissipation capacity. 


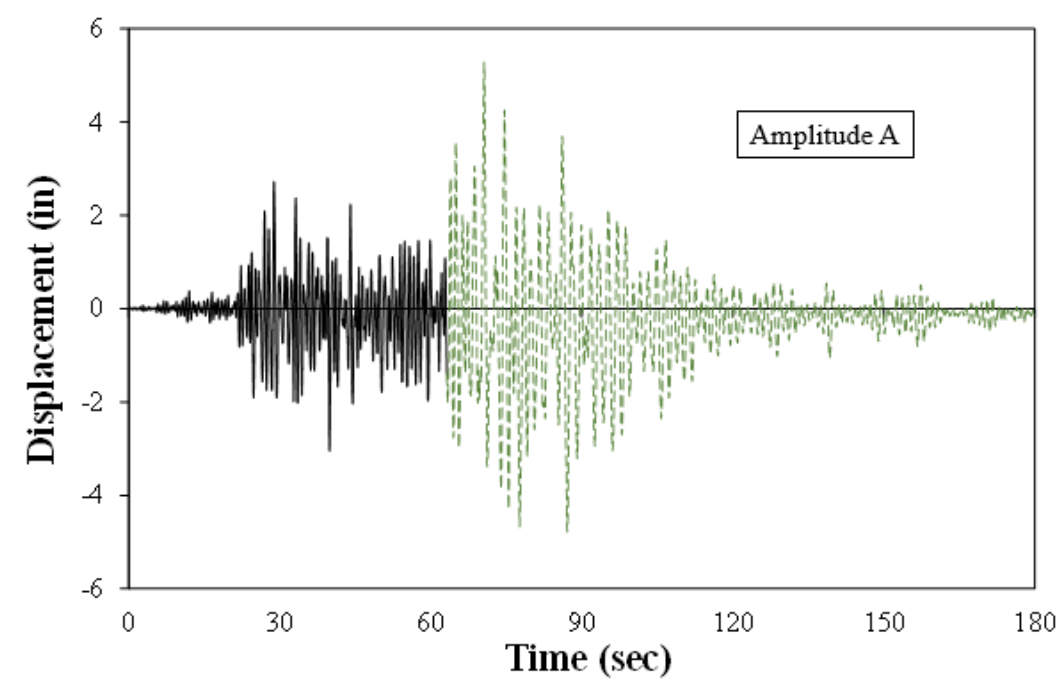

Figure 3.81 Column displacement history for column C5S1-M

Table 3.16 Summary of the measured performance for C5S1-M

\begin{tabular}{c|c}
\hline Property & Amplitude A \\
\hline $\begin{array}{c}\text { Max. Displacement } \\
\text { in. (mm) }\end{array}$ & $5.3(134.2)$ \\
\hline $\begin{array}{c}\text { Max. Drift Ratio } \\
(\%)\end{array}$ & 5.50 \\
\hline $\begin{array}{c}\text { Max. Displacement } \\
\text { Ductility Level }(\mu)\end{array}$ & 4.2 \\
\hline $\begin{array}{c}\text { Residual } \\
\text { Displacement in. } \\
(\mathrm{mm})\end{array}$ & $0.14(3.6)$ \\
\hline $\begin{array}{c}\text { Max. Base Shear } \\
\text { kips (kN) }\end{array}$ & $21.6(96.2)$ \\
\hline
\end{tabular}

The maximum displacement for each earthquake motion amplitude, and its corresponding drift ratio and ductility level are presented in Table 3.16. These values are the peak displacement values and do not correspond to the peak lateral force values, which are also listed in Table 3.8. Reported displacement ductility levels are based on the effective yield displacement, which was found to be $1.27 \mathrm{in}$. This value was computed using the reduce stiffness method assuming an elasto-plastic idealization. Even though 
C4S1-L was detailed with a short lap splice, strain gauges placed on the extreme starter bars reached the yield strain. Since the specimens were already damaged, it allowed the spliced bars to engage and transfer the forces, and so yielding of longitudinal reinforcement occurred. The displacement history for column C5S1-M is shown in Figure 3.81. It also indicates the moment at which the lap-spice failure occurred (green dashed line). On the other hand, it is seen in Figure 3.81 and Table 3.16 that column C5S1-M experienced little residual displacement for motion amplitude A.

\subsubsection{Dynamic Properties}

Low-level white noise signals, as well as pulse tests, were performed before and in between earthquake motion amplitudes to monitor the change in the dynamic properties due to damage progression in the specimen. Natural periods were determined from Fourier analysis of the accelerations measured at the top of the column. The damping ratio was calculated from the free vibration portion of the accelerograms recorded at the top of the specimen using the decrement logarithmic method. Since the free-vibration portion of the accelerogram does not have a perfect exponential decay, the accelerogram was divided into various intervals for which a damping ratio was calculated. Then, a mean value was calculated for the damping ratio. A summary of the computed periods and mean damping ratio values and standard deviations are presented in Table 3.17. The uncracked natural period of the column model was $0.35 \mathrm{sec}$; however, it is not included in Table 3.17, because specimen C5S1-M was tested in a damaged condition due to high amplitude signal from the shake table system before running the correct motion. Therefore, the initial period reported in Table 3.17 represents the cracked 
natural period. From the results, it is clear that the computed natural periods were lengthened with the increased level of excitation, what confirmed the deterioration of column stiffness. The variation in damping followed the same trend, i.e., it increased with the progression of the damage from $2.5 \%$ to $5.0 \%$. In general, good agreement was observed between the results of both tests.

Table 3.17 Measured dynamic properties for C5S1-M

\begin{tabular}{c|c|c|c|c}
\hline \multirow{2}{*}{ Sequence } & \multicolumn{2}{|c|}{ Period, $T_{n}(\mathrm{sec})$} & \multicolumn{2}{c}{ Damping ratio, $\zeta(\%)$} \\
\cline { 2 - 5 } & Pulse test & W-N random test & $\mu_{X}$ & $\sigma_{X}$ \\
\hline Initial & 0.46 & 0.46 & 2.5 & 0.44 \\
\hline amplitude A & 0.68 & 0.70 & 5.0 & 0.27 \\
\hline
\end{tabular}

\subsubsection{Strains}

The recorded steel strain histories of two strain gauges on the dowel bars are shown in Figure 3.82 and Figure 3.83. Based on the measured and known values, peak steel strain profiles were derived and plotted (Figure 3.84) along the splice length. Lateral strength degradation coincided with the initiation of bond deterioration along the splice length, and bond deterioration was most significant at the bottom of the splice length where the maximum moment occurred. It is worth noting that bond deterioration occurred during motion amplitude A for this column, whereas for Column C4S1-L occurred during motion amplitude B. This result can be attributed to the large number of strain cycles imposed by subduction records compared to crustal records. 


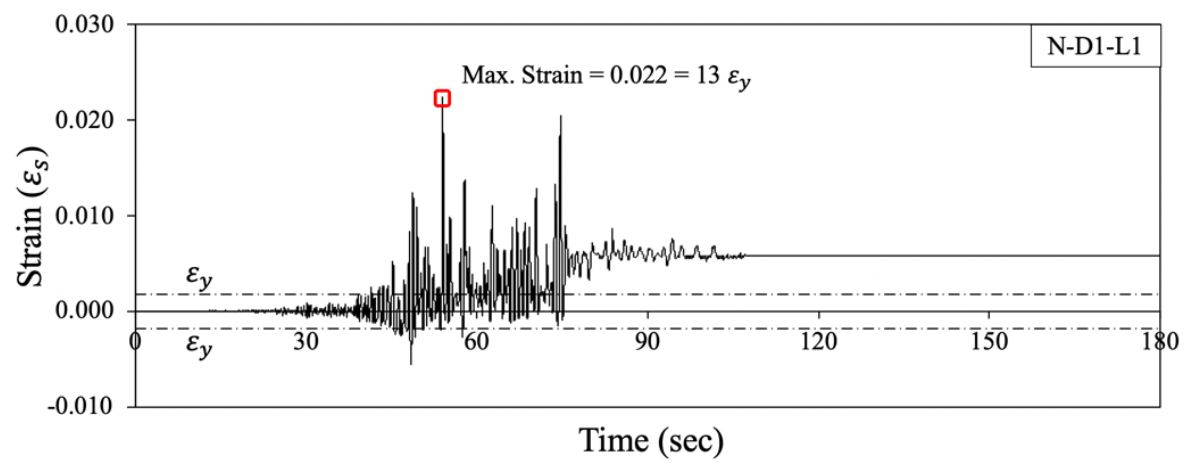

Figure 3.82 Steel strain history for strain gauge N-D1-L2 for Column C5S1-M during motion amplitude $\mathrm{A}$

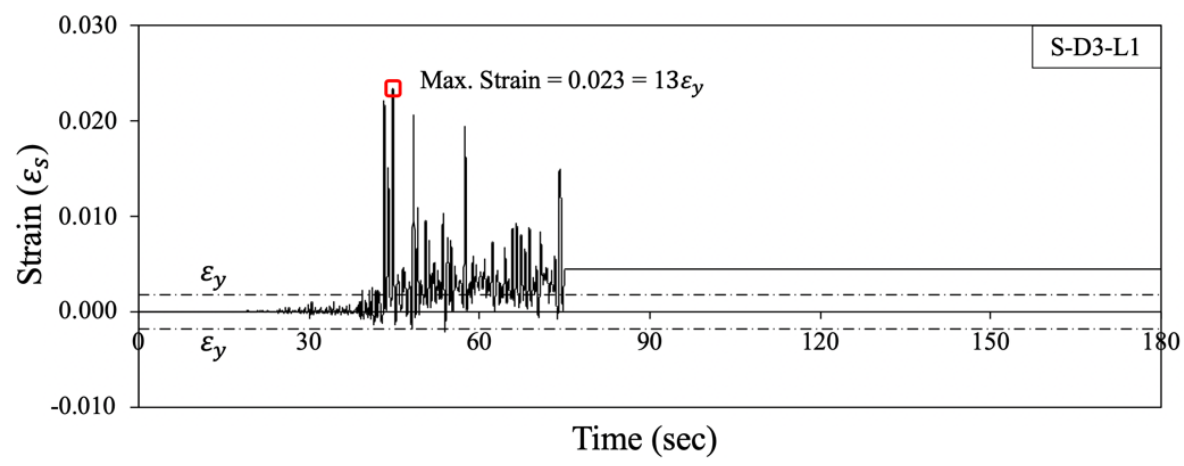

Figure 3.83 Steel strain history for strain gauge S-D3-L1 for Column C5S1-M during motion amplitude $\mathrm{A}$

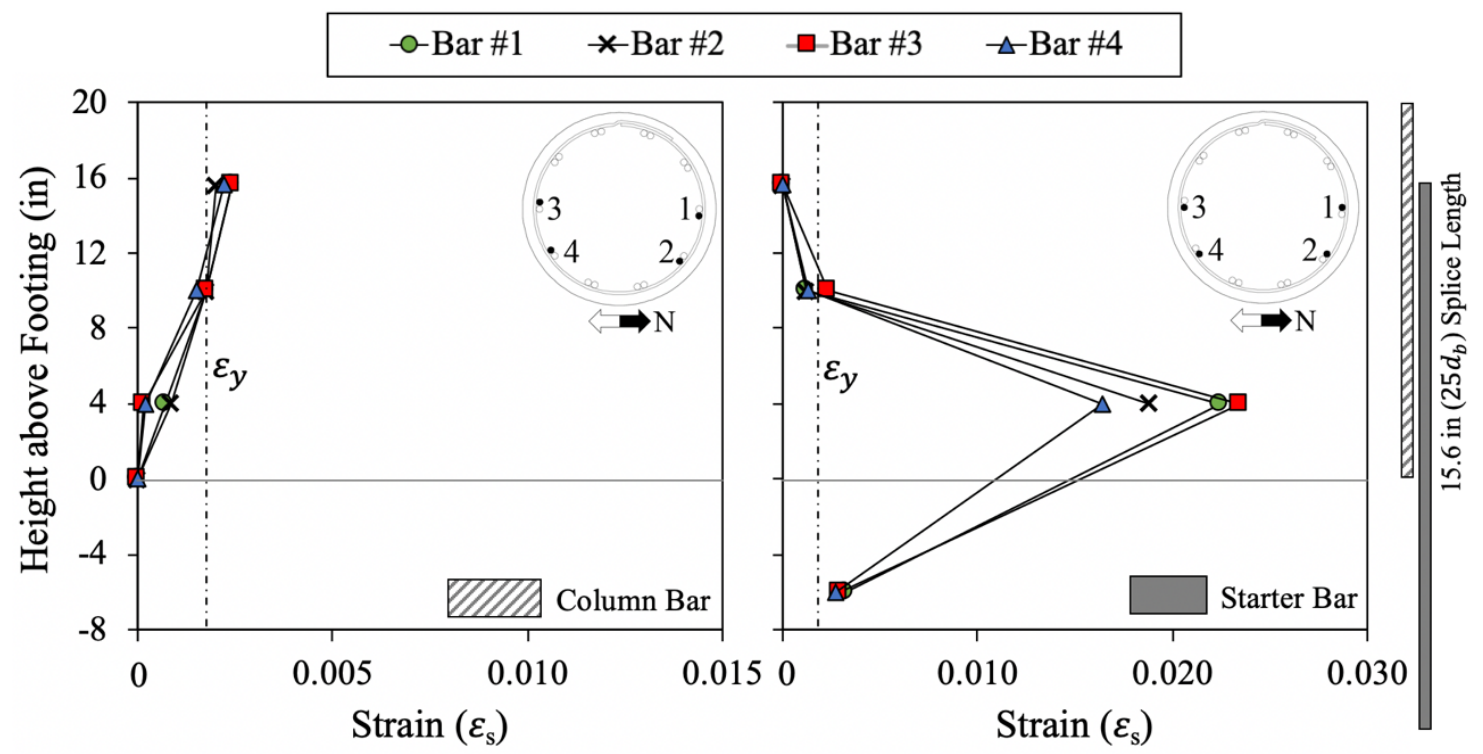

Figure 3.84 Peak steel strain profile for Column C5S1-M 


\subsubsection{Curvature}

As mentioned before, linear variable displacement transducers (LVDTs) were used to measure the average curvature along the height of the column. The peak curvature profile along the height is shown in Figure 3.85. The points in the curvature profiles represent the curvature measured at the specific location and in correspondence to the maximum and minimum peak values of column displacement. It is seen in the figure that the average curvatures calculated at the column-footing interface were orders of magnitude higher because they include contributions from curvature and bond slip. Thus, the relative magnitudes demonstrate that the deformations from bond slip control the response at the interface, validating the visual observations of bond slip failure along the splice region of the column during the second motion.

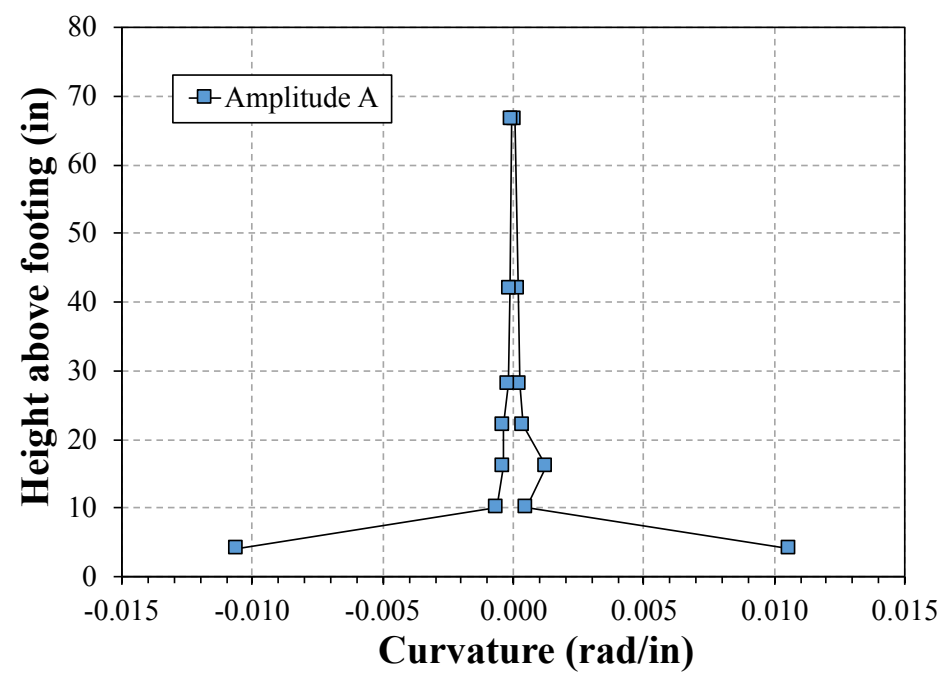

Figure 3.85 Average curvature profiles in column C5S1-M

\subsubsection{Axial Forces}

Although the experiments were designed to keep the same level of axial force during the test sequence, some variations were observed during testing. The axial force 
history for specimen C5S1-M is shown in Figure 3.86. The target force was 75 kips (334 $\mathrm{kN}$ ) for motion amplitude $\mathrm{A}$, and the maximum deviation from the target axial force was $-22.6 \%$ and $+57.3 \%$. The variations of the axial force may be attributed to the change in axial loads in the treaded rods as the specimen displaced. Normally, the accumulators compensate for this, but the hinging action of the load transfer beam created a less stiff axial load system. The less stiff system caused the hydraulic ram to displace further and faster than its ability to maintain constant axial load. However, the high deviation from the target axial during motion amplitude B was due to the crushing of concrete core following the lap-splice failure, which led to loss of axial capacity and relaxation of the post-tensioning rods.

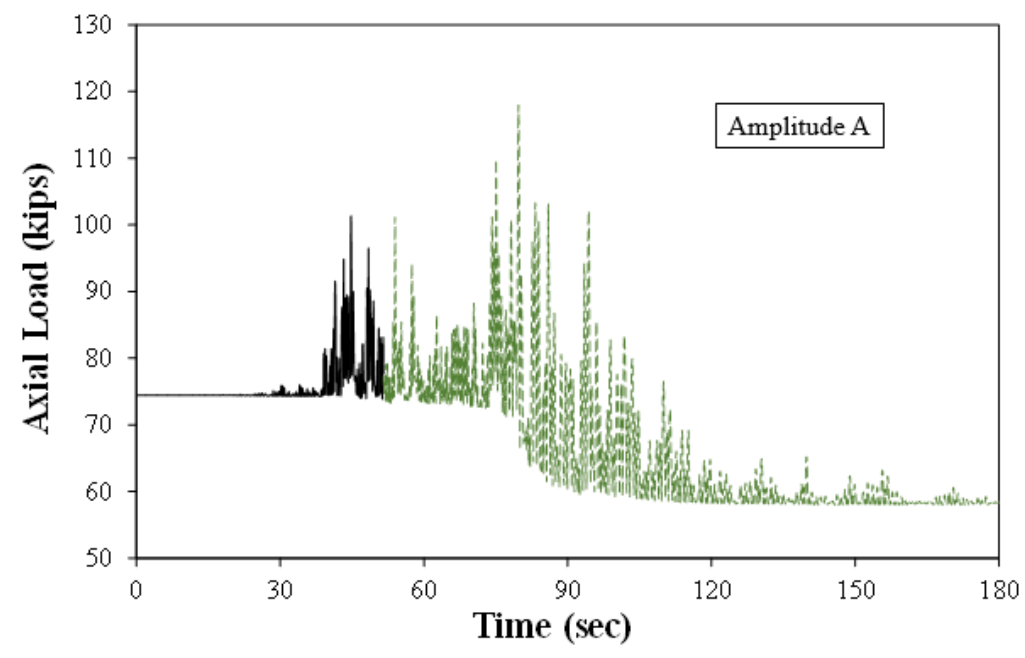

Figure 3.86 Axial force history for column C5S1-M

\subsubsection{Column C6S2-M}

Column C6S2-M was the sixth column tested. It was subjected to a subduction ground motion from the 2010 Maule earthquake (Curico X record). As compared to specimen C5S1-L, this specimen was designed with a longer lap splice at the hinge zone 
with the aim of contrast the damage differences between reinforcement detailing. Test results and discussion are presented in the following subsections.

\subsubsection{Progression of Observed Damage}

Column C6S2-M-M was only subjected to motion amplitude A. Column damage consisted of spalling of the concrete cover up to 4 inches in height on the north and south sides along with vertical cracks along the lap splice at the column base. After the test, crushing of concrete at the column base was observed, exposing column reinforcement on the north side only (Figure 3.87). In contrast to the observed damage in column C5S1M, column C6S2-M did not show splitting cracks at the hinge zone since this specimen was provided of a longer spliced length. Furthermore, this specimen exhibited less damage than $\mathrm{C} 2 \mathrm{C}-\mathrm{M}$ for the same motion amplitude, and it was due to the rocking at the cold joint between the column and footing. Even though the specimen was able to sustain an increased earthquake level, it was decided to save the column for retrofit purposes.

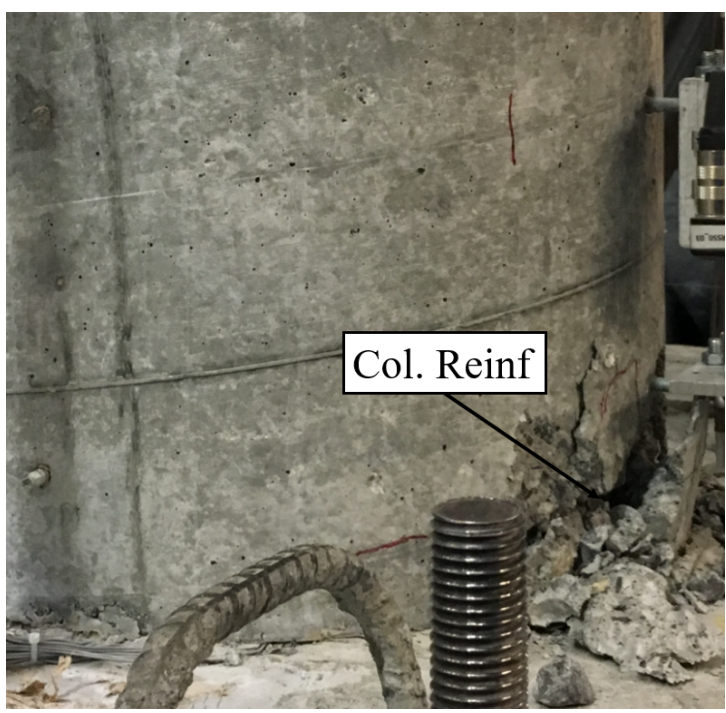

a)

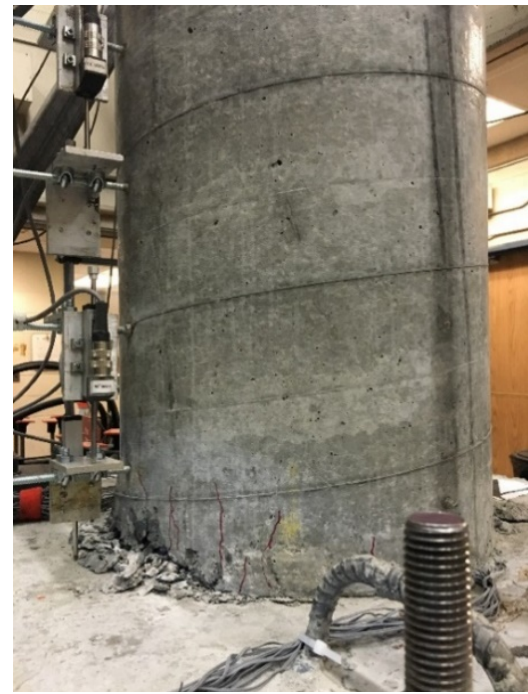

b)

Figure 3.87 Damage state of column C6S2-M after applying motion amplitude A. a) North-east view, b) south-east view 
As expected for a cantilever column, the damage was fully localized to the lower part of the column in the plastic hinge region. Figure 3.88 shows the specimen at completion of testing.

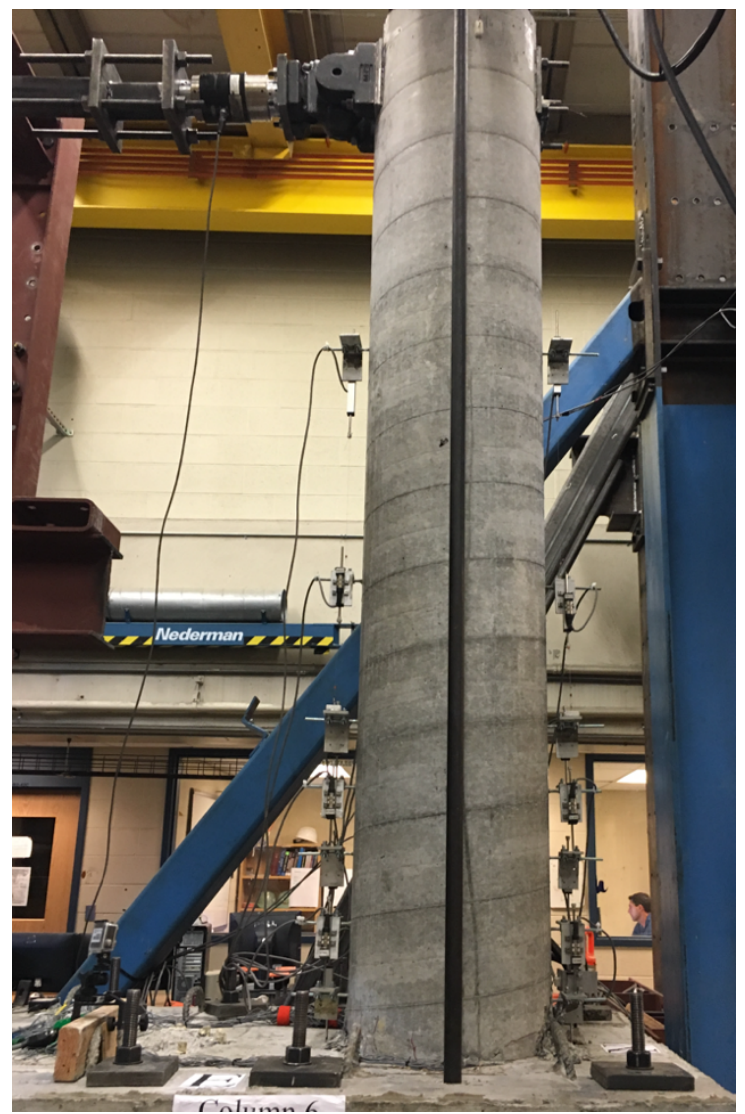

Figure 3.88 Final state of column C6S2-M

\subsubsection{Force-Displacement Relationship}

The measured hysteresis curve for column C6S2-M for motion amplitude A is shown in Figure 3.89. Damage states are also identified on the force-displacement response histories. As was mentioned previously, the specimen was loaded in the northsouth direction. This means that positive displacement direction corresponds to the north direction, and negative displacement direction is towards the south direction. 
Based on the experimental results, column $\mathrm{C} 2 \mathrm{C}-\mathrm{M}$ showed a hysteretic response typical of flexural behavior. Despite the design deficiencies of column C6S2-M, namely, lap splice at hinge zone, low ratio of transverse reinforcement and no seismic detailing, C6S2-M showed a moderate ductile behavior as compared to the other two specimens in the same test series for amplitude A, with more stable hysteretic loops and higher energy dissipation capacity, similar to $\mathrm{C} 2 \mathrm{C}-\mathrm{M}$ in the first motion amplitude. C6S2-M reached a peak lateral load of 19.9 kip at a displacement ductility of $\mu=3.1$ ( $\Delta=2.9$ in). After the earthquake motion, there was no significant loss in column strenght.

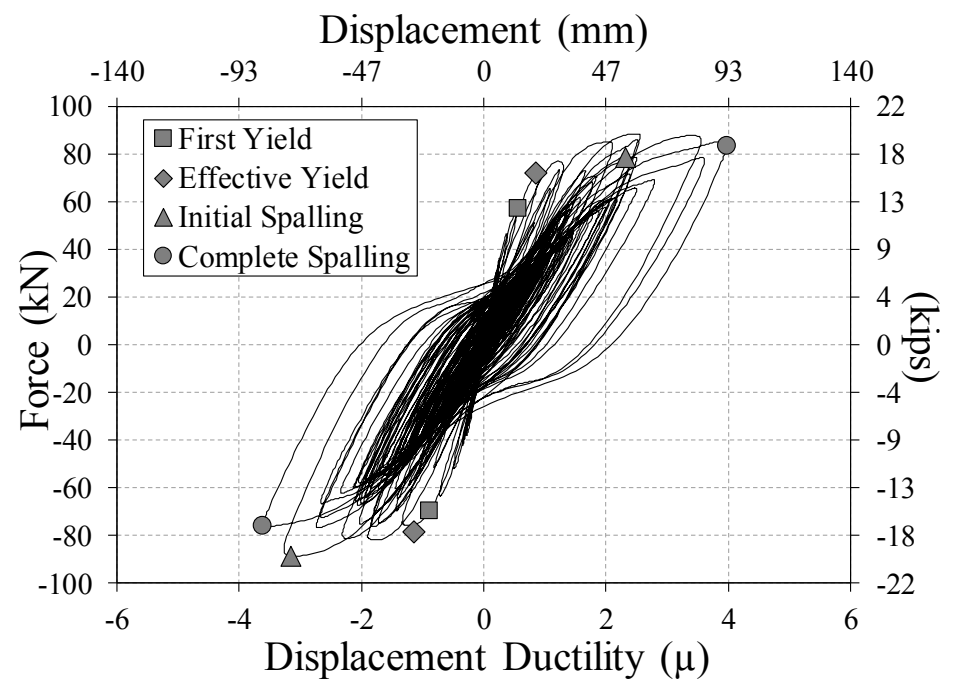

Figure 3.89 Hysteretic response for column C6S2-M during motion amplitude A

The maximum displacement for each earthquake motion amplitude, and its corresponding drift ratio and ductility level are presented Table 3.18. These values are the peak displacement values and do not correspond to the peak lateral force values, which are also listed in Table 3.18. Reported displacement ductility levels are based on the effective yield displacement, which was found to be $0.92 \mathrm{in}$. This value was computed using the reduce stiffness method assuming an elasto-plastic idealization. The 
displacement history of column C6S2-M is shown in Figure 3.90. On the other hand, it is seen in Figure 3.90 and Table 3.18 that column C2C-M experienced little residual displacement after motion amplitude A. Final residual displacement after motion amplitude $\mathrm{C}$ was 0.02 in.

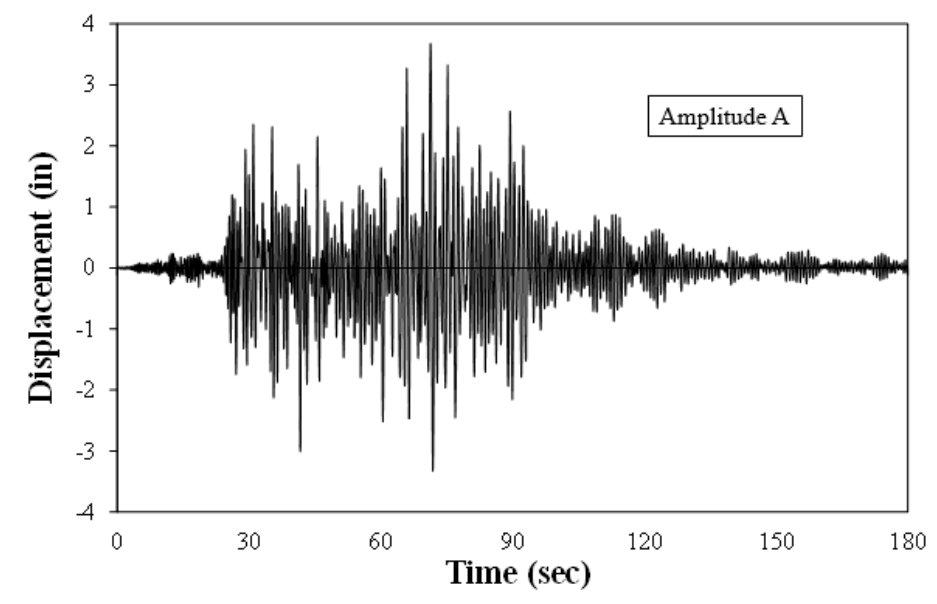

Figure 3.90 Column displacement history for column C6S2-M

Table 3.18 Summary of the measured performance for C6S2-M

\begin{tabular}{c|c}
\hline Property & Amplitude A \\
\hline $\begin{array}{c}\text { Max. Displacement } \\
\text { in. (mm) }\end{array}$ & $3.7(93.4)$ \\
\hline $\begin{array}{c}\text { Max. Drift Ratio } \\
(\%)\end{array}$ & 3.83 \\
\hline $\begin{array}{c}\text { Max. Displacement } \\
\text { Ductility Level }(\mu)\end{array}$ & 4.0 \\
\hline $\begin{array}{c}\text { Residual } \\
\text { Displacement in. } \\
(\mathrm{mm})\end{array}$ & $0.02(0.5)$ \\
\hline $\begin{array}{c}\text { Max. Base Shear } \\
\text { kips (kN) }\end{array}$ & $19.9(88.7)$ \\
\hline
\end{tabular}

\subsubsection{Dynamic Properties}

Low-level white noise signals, as well as pulse tests, were performed before and in between earthquake motion amplitudes to monitor the change in the dynamic 
properties due to damage progression in the specimen. Natural periods were determined from Fourier analysis of the accelerations measured at the top of the column. The damping ratio was calculated from the free vibration portion of the accelerograms recorded at the top of the specimen using the decrement logarithmic method. Since the free-vibration portion of the accelerogram does not have a perfect exponential decay, the accelerogram was divided into various intervals for which a damping ratio was calculated. Then, a mean value was calculated for the damping ratio. A summary of the computed periods and mean damping ratio values and standard deviations are presented in Table 3.19. From the results, it is clear that the computed natural periods were lengthened with the increased level of excitation, what confirmed the deterioration of column stiffness. The variation in damping followed the same trend, i.e., it increased with the progression of the damage from $1.4 \%$ to $4.4 \%$. In general, good agreement was observed between the results of both tests.

Table 3.19 Measured dynamic properties for C6S2-M

\begin{tabular}{c|c|c|c|c}
\hline \multirow{2}{*}{ Sequence } & \multicolumn{2}{|c|}{ Period, $T_{n}(\mathrm{sec})$} & \multicolumn{2}{c}{ Damping ratio, $\zeta(\%)$} \\
\cline { 2 - 5 } & Pulse test & W-N random test & $\mu_{X}$ & $\sigma_{X}$ \\
\hline Initial & 0.33 & 0.33 & 1.4 & 0.18 \\
\hline amplitude A & 0.56 & 0.57 & 4.4 & 0.04 \\
\hline
\end{tabular}

\subsubsection{Strains}

The recorded steel strain histories of two strain gauges on the dowel bars are shown in Figure 3.91 and Figure 3.92. Recall that this column was detailed with a longer splice length at its bottom compared to C4S1-L and C5S1-M. The used strain gauges were able to record most of the strain measurements, though some data is missing 
because they malfunctioned, most likely due to damage during construction or during testing. There was some initial strain in the reinforcement prior to shake table testing due to the applied axial loading. For Column C6S2-M, three strain gauges were affixed to the column longitudinal bars and to the starter bars anchored into the footing. Also, it is known that longitudinal strain is equal to zero at the ends of the longitudinal reinforcement bars. Based on the measured and known values, peak steel strain profiles were derived and plotted (Figure 3.93) along the splice length. Even though the column was subjected to a large number of strain cycles compared to C4S1-L, the increase in splice length prevented the initiation of bond deterioration and therefore there was no degradation in lateral strength. A maximum strain of $0.037 \mathrm{in} / \mathrm{in}$ was measured in the south dowel bar during motion amplitude A, which corresponds to 20.7 times the yield $\operatorname{strain}\left(\varepsilon_{y}\right)$.

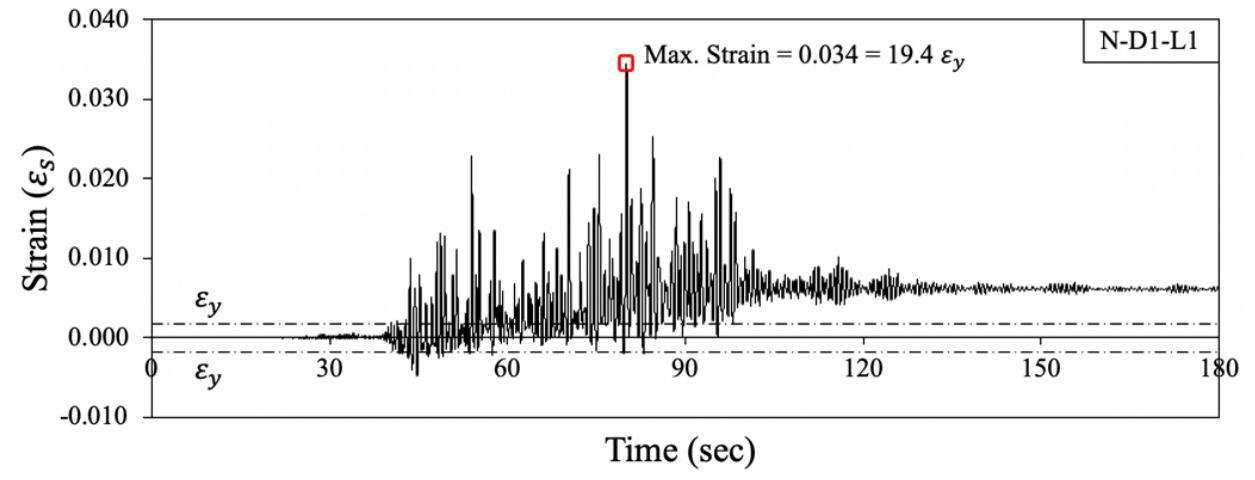

Figure 3.91 Steel strain history for strain gauge N-1-L2 for column C6S2-M during motion amplitude A 


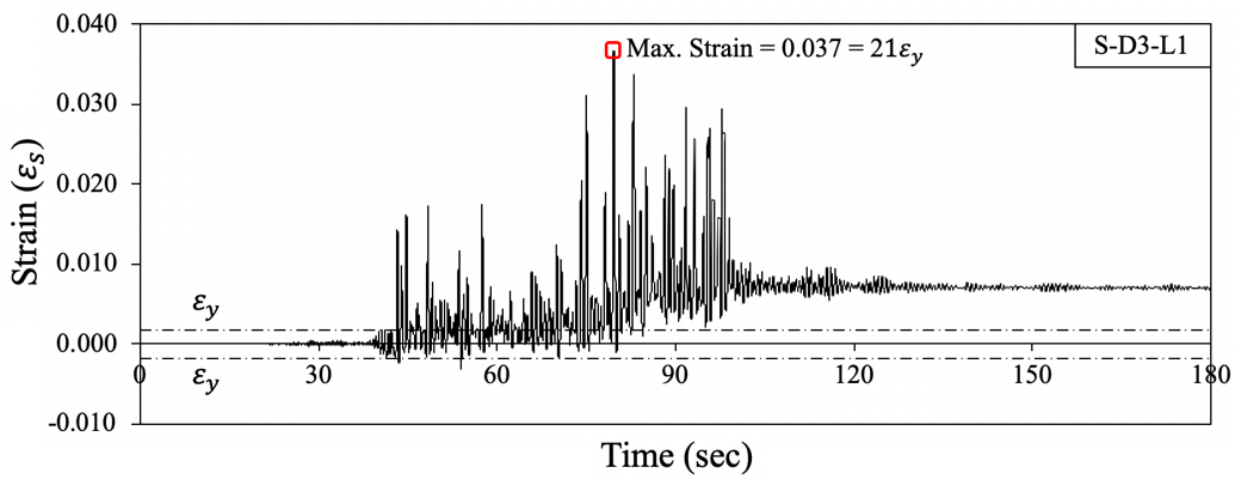

Figure 3.92 Steel strain history for strain gauge S-3-L1 for column C6S2-M during motion amplitude A

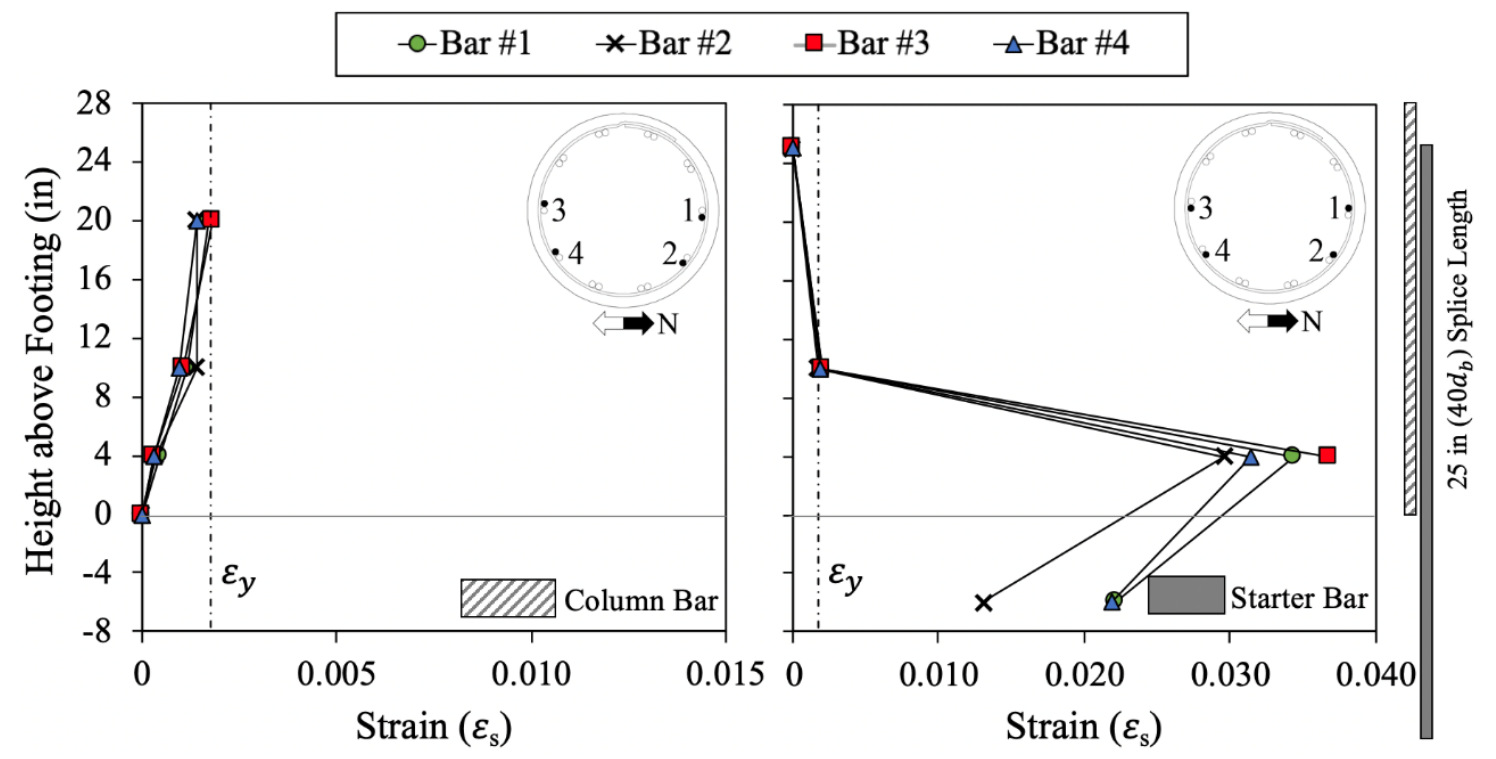

Figure 3.93 Peak steel strain profile for column C6S2-M

\subsubsection{Curvature}

As mentioned before, linear variable displacement transducers (LVDTs) were used to measure the average curvature along the height of the column. The peak curvature profile along the height is shown in Figure 3.94. The points in the curvature profiles represent the curvature measured at the specific location and in correspondence to the maximum and minimum peak values of column displacement. During motion 
amplitude A, the low average curvatures in sections above the interface and within the splice agree with the minor damage observed in the column. The average curvatures calculated at the column-footing interface were orders of magnitude higher because they include contributions from curvature and bond slip. However, these values are lower compared to Specimen C5S1-M and agree with the minor damage observed in Column C6S2-M. This result demonstrates that the increase in the splice length prevented the development of bond slip failure along the splice region.

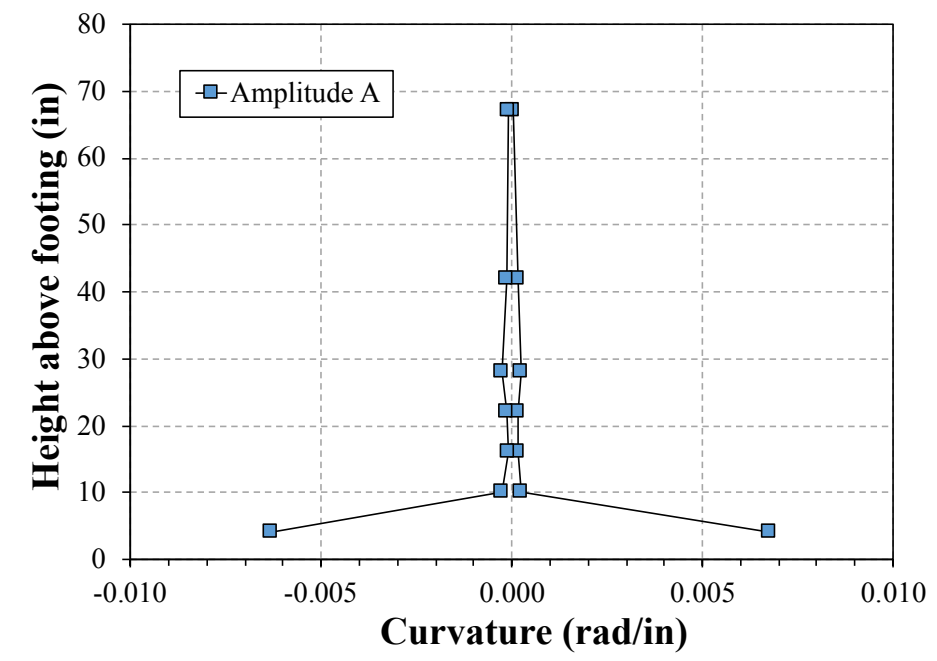

Figure 3.94 Average curvature profiles in column C6S2-M

\subsubsection{Axial Forces}

Although the experiments were designed to keep the same level of axial force during the test sequence, some variations were observed during testing. The axial force history for specimen C6S2-M is shown in Figure 3.95. The target force was 75 kips (334 $\mathrm{kN}$ ), and the maximum deviation from the target axial force was $-3.1 \%$ and $+42.6 \%$. The variations of the axial force may be attributed to the change in axial loads in the treaded rods as the specimen displaced. Normally, the accumulators compensate for this, but the 
hinging action of the load transfer beam created a less stiff axial load system. The less stiff system caused the hydraulic ram to displace further and faster than its ability to maintain constant axial load.

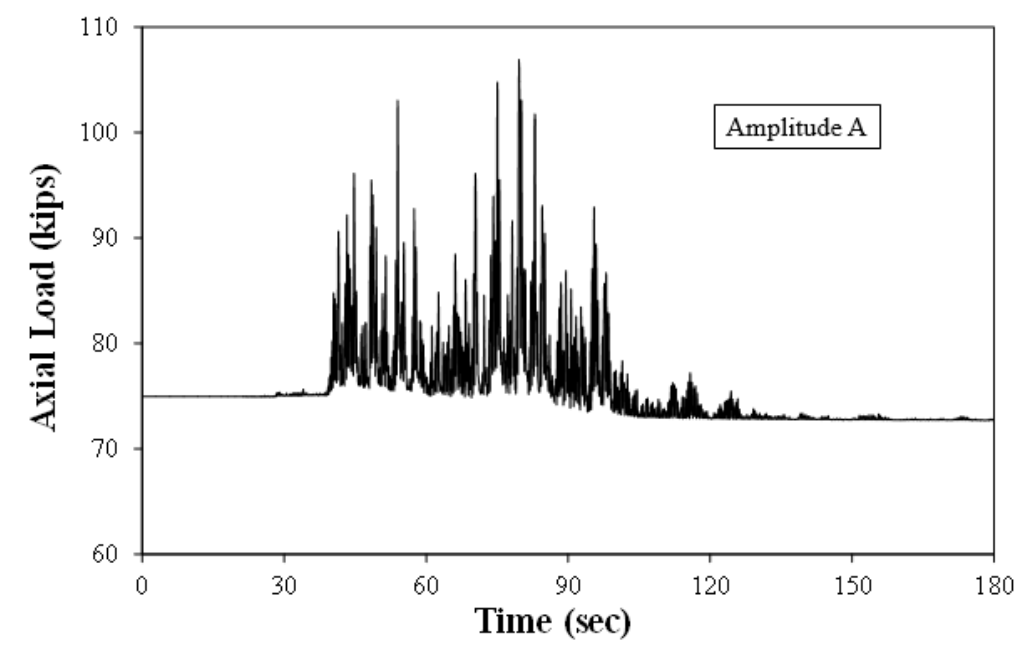

Figure 3.95 Axial force history for column C6S2-M

\subsection{Column Comparisons}

Since all the columns had the same dimensions, it was possible to make comparisons between the various columns tested. This section compares the test results from the experimental program. Recall that the main objective of the experimental program was to study the effect of subduction zone earthquake demands on the performance of substandard RC bridge columns. The following subsections present global and local behavior comparisons.

\subsubsection{Backbone Curve Comparison}

Backbone curves were used to visualize the effects of subduction zone earthquakes in the overall load and displacement capacity that the specimens exhibited. Figure 3.96 compares the backbone curves for each ground motion amplitude in terms of ductility. In 
the first motion amplitude, specimens with continuous longitudinal reinforcement exhibited moderate displacement ductility around $\mu=4$ and almost similar lateral strength, but higher stiffness degradation was exhibited by column C2C-M. After the second motion amplitude, none of the specimens were able to reach the target displacement ductility of 6 , mainly due to the degraded lateral stiffness. However, only specimens $\mathrm{C} 2 \mathrm{C}-\mathrm{M}$ and $\mathrm{C} 3 \mathrm{C}-\mathrm{T}$ failed during motion amplitude B due to longitudinal bar buckling, therefore reaching their maximum displacement ductility. This result may indicate that subduction ground motions reduce the maximum displacement ductility at failure and can be atrributed to the large number of applied cycles. Degradation in lateral strength after both motion amplitudes was also greater for columns subjected to sunduction ground motion records, dropping about $20 \%$ of their peak lateral strength.

In the second test series, two observations can be made based on the lap-splice length. Comparing specimens C4S1-L and C5S1-M underscores the difference in performance caused by the increased duration of the ground motion. Responses of both specimens are characterized as non-ductile, that is, no displacement ductility was exhibited. Difference in the peak lateral strength between these two specimens was likely a result of the fluctuation in the applied axial load in C5S1-M. Up to a displacement ductility of $\mu=2$, both specimens exhibited a similar effective lateral stiffness. However, the increase in the number of loading cycles triggered the lap-splice failure mode in column C5S1-M during the first ground motion amplitude, and similar to those described in the literature (Priestley et al., 1996). In the second motion amplitude, C4S1-L lost its lateral load-carrying capacity due to lap splice failure. The moderate ductile performance 
of C6S2-M was the result of a long, but still deficient, lap splice length $\left(40 \mathrm{~d}_{b}\right)$. This specimen reached a displacement ductility of $\mu=4$ and had a decrease in its lateral strength of $16 \%$ of the peak lateral load. As can be seen in Figure 3.96(a), the backbone curve for $\mathrm{C} 6 \mathrm{~S} 2-\mathrm{M}$ was similar to the one for $\mathrm{C} 2 \mathrm{C}-\mathrm{M}$. Again, the difference in lateral strength between these two specimens may be attributed to the fluctuation in the applied axial load in $\mathrm{C} 6 \mathrm{~S} 2-\mathrm{M}$.

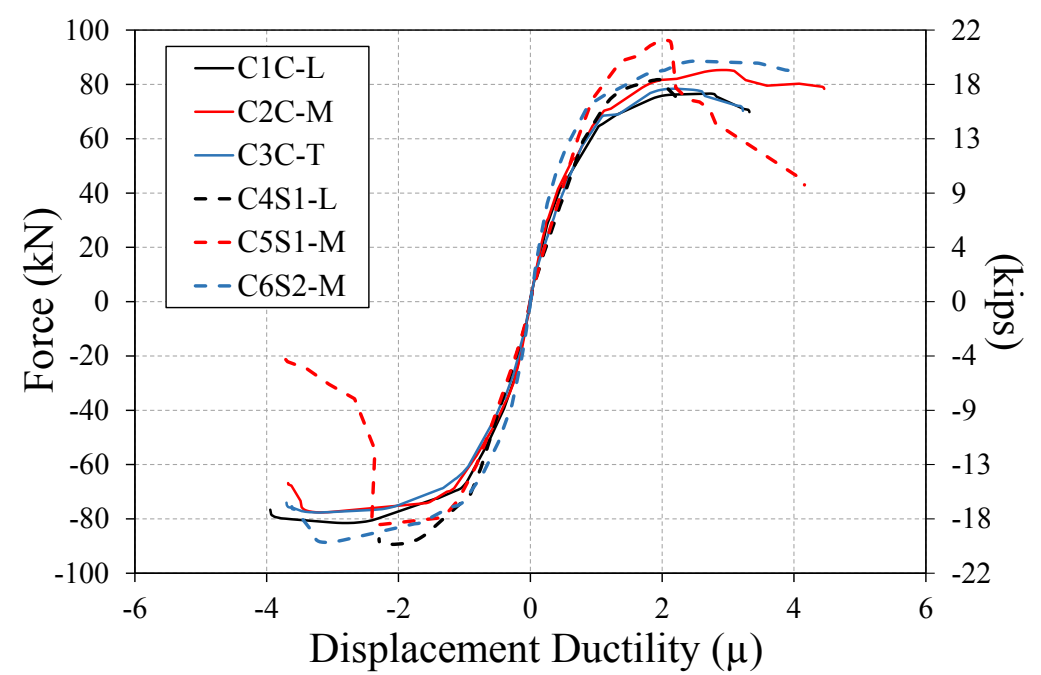

a)

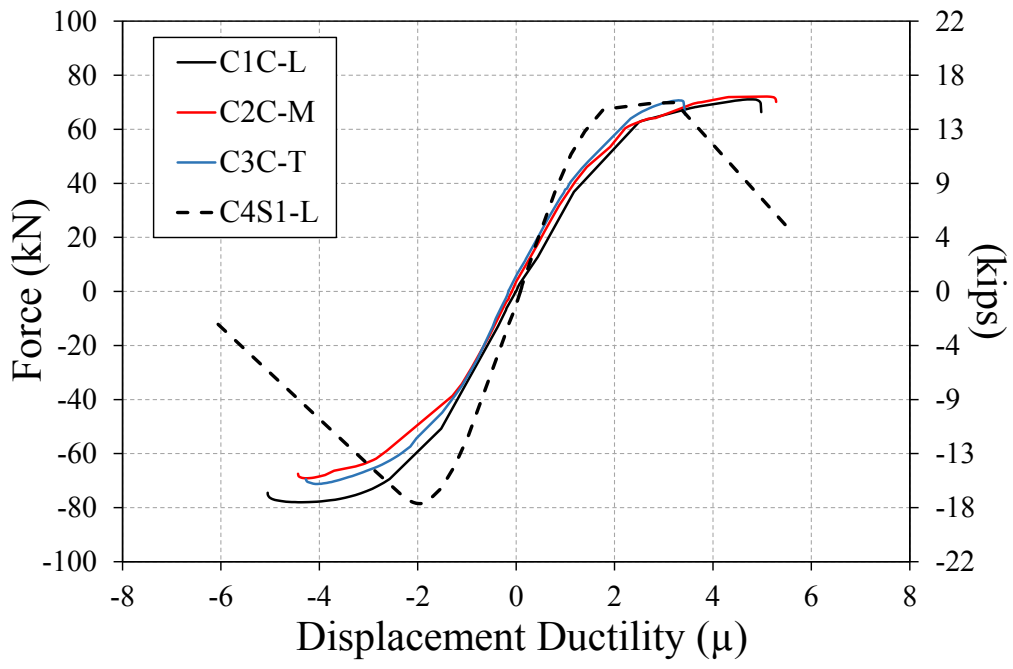

b)

Figure 3.96 Backbone curves comparison for all specimens. a) Amplitude A, b) Amplitude B 161 


\subsubsection{Energy Dissipation and Stiffness Degradation}

RC bridge components exhibit some level of stiffness degradation under seismic events. This key parameter is usually the result of cracking, cover spalling, loss of bond, crushing of concrete, and yielding and buckling of reinforcement. The level of stiffness degradation depends, among others, on the loading history (e.g., number of cycles) and, as a result, it will influence the natural period of the structure. In this study, the effective lateral stiffness $\left(k_{\text {eff }}\right)$ was used as the stiffness degradation parameter and was estimated from the recorded force and displacement values during each ground motion amplitude. Two large displacement cycles were chosen from the column displacement history. The effective lateral stiffness was then computed by measuring the slope of the line that connected the maximum and minimum displacements in each cycle in the hysteretic curves. Figure 3.97 shows the degradation in the lateral stiffness for the tested columns after each motion amplitude. Specimens subjected to subduction ground motions exhibited higher degradation than the ones under crustal record after the first motion amplitude. This can be attributed to extend of the spalled region at hinge, especially for specimen $\mathrm{C} 2 \mathrm{C}-\mathrm{M}$. After motion amplitude B, specimens of the first test series showed a slightly decrease in the lateral effective stiffness but still greater for subduction demands. In the second test series, specimen C5S1-M exhibited a higher degradation in lateral stiffness due to lap splice failure that led to lateral instability, after motion amplitude A. Even though specimen C4S1-L exhibited a loss in its lateral effective stiffness due to lap splice failure, this situation was achieved in the second ground motion amplitude. These 
outcomes reveal that subduction ground motions also affect the stiffness degradation parameter.

The energy dissipation was calculated by integrating the area within the loops of the hysteretic curves. Figure 3.98 shows the cumulative energy dissipation versus the number of cycles for all specimens for each ground motion amplitude. As can be seen, subduction ground motions lead to more energy dissipation that the crustal record. Thus, besides the column's hysteretic behavior, the increased number of loading cycles imposed by subduction records greatly increase the cumulative energy dissipated. Similar trend is observed between ground motion amplitudes, accompanied by a slightly increase in the cumulative energy dissipated energy. For columns subjected to subduction ground motions, specimen C5S1-M dissipated less energy due to lap splice failure. Conversely, an increase in the spliced length at the hinge zone resulted in an energy dissipation capacity similar to specimen with continuous longitudinal reinforcement.

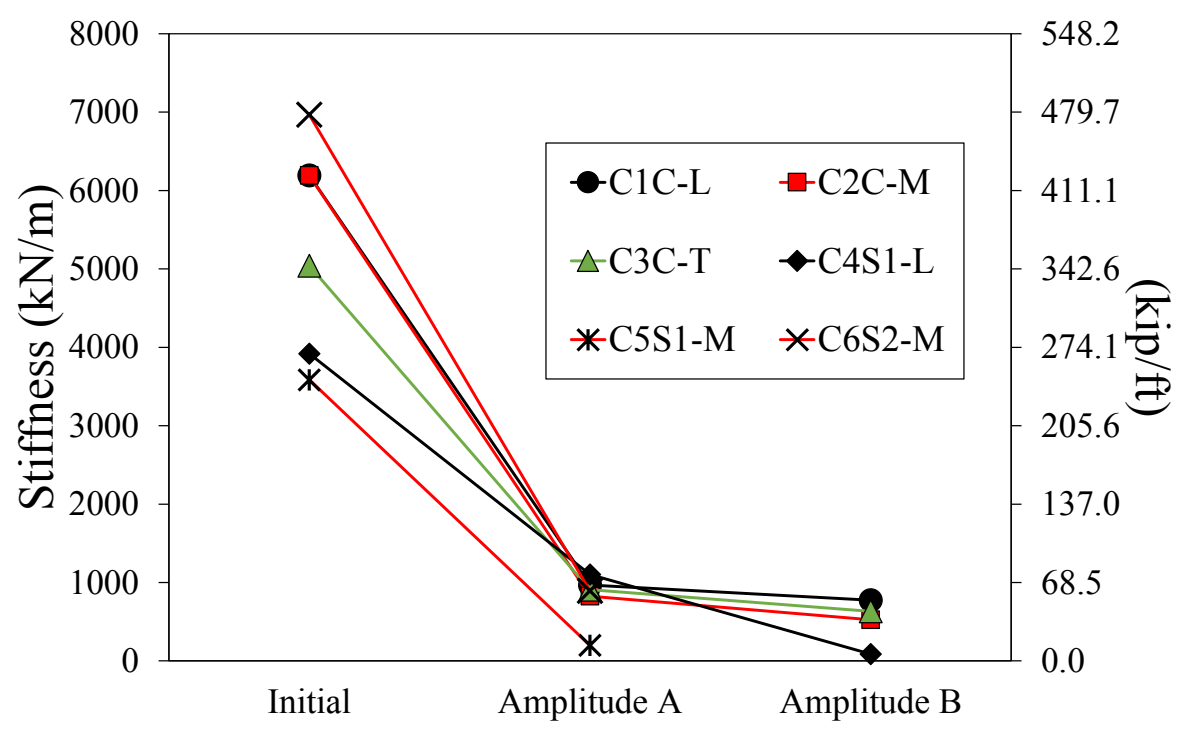

Figure 3.97 Lateral stiffness degradation after each ground motion amplitude for all specimens. a) Amplitude A, b) Amplitude B 


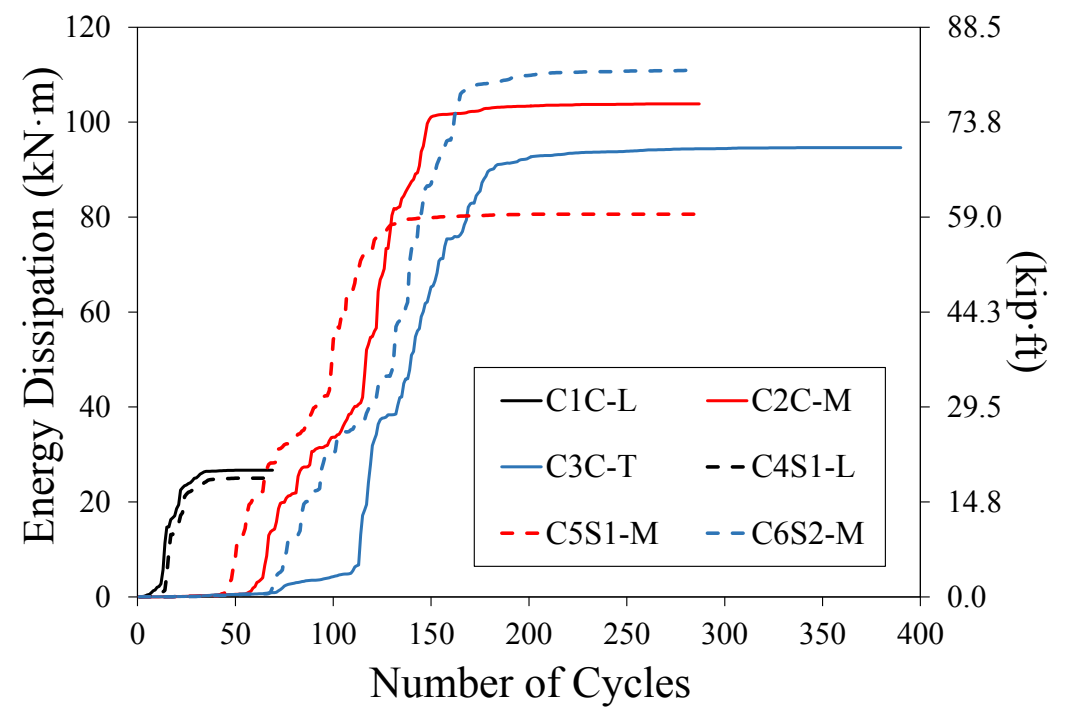

a)

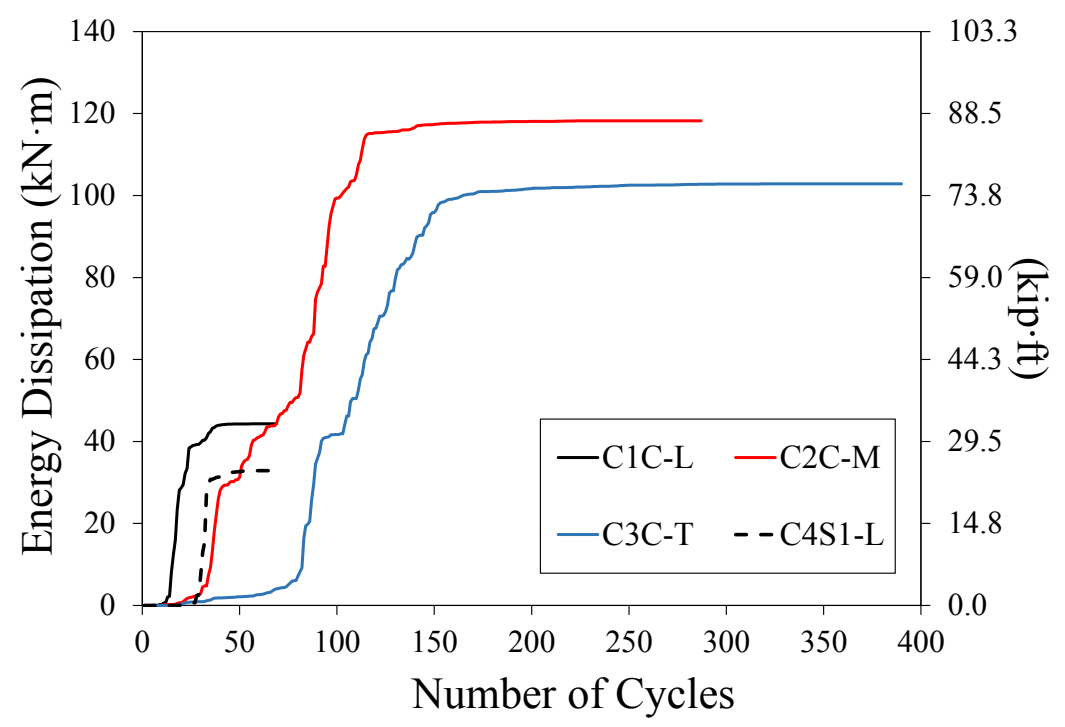

b)

Figure 3.98 Energy dissipation comparison for all specimens. a) Amplitude A, b) Amplitude B

\subsubsection{Cumulative Damage}

Load reversals imposed by earthquakes tend to increment the damage and degradation of the strength and stiffness. They also affect the displacement capacity of structural elements. A correlation can be assumed between damage and the number of 
inelastic cycles underwent by structural component (Stephens \& Yao, 1987). Several models can be found in the literature to address this relationship, but for simplicity, the Cumulative Displacement Ductility Factor (CDD) (Park, 1989) was used as damage index. This index is computed by accumulating the ratio of plastic deformation under an excursion to the yield displacement, given by Eq. (2.5)

$$
C D D=\sum_{i=1}^{N} \frac{\Delta_{p i}}{\Delta_{y}}=\sum_{i=1}^{N} \mu_{i}
$$

Where $\mathrm{N}$ corresponds to the number of inelastic cycles, equal to the number of cycles above ductility one $(\mu=1.0)$. Care should be taken when assessing the effects of several load reversals on the structure via CDD index. A structure undergoing a different number of cycle histories can result in same CDD index. Pre-peak and all inelastic excursion cycles were obtained to study the effect of each demand on column performance. Table 3.20 lists the number of inelastic cycles for pre-peaks and all excursion, as well as the CDD index for each motion amplitude for all columns. From the table, it is clear that subduction earthquakes records imposed a larger number of inelastic cycles than the crustal motion. The hysteresis of each column resulted in CDD index much greater for subduction records. These indexes are in agreed with the visual observation were more damage was observed in specimens tested under subduction records. Furthermore, the increase in the number of inelastic cycles affects the material behavior, resulting in core concrete damage and buckling of longitudinal reinforcement. These results confirm the fact that subduction earthquakes affect the mode of failure of bridge columns. 
Table 3.20 Cumulative Displacement Ductility

\begin{tabular}{c|c|c|c|c}
\hline \multirow{2}{*}{ Specimen } & Amplitude & $\begin{array}{c}\text { N.I.C } \\
\text { (Pre-peaks) }\end{array}$ & NIC & CDD \\
\hline \multirow{2}{*}{ C1C-L } & $\mathrm{A}$ & 10 & 20 & 63 \\
\cline { 2 - 5 } & $\mathrm{B}$ & 8 & 16 & 72 \\
\hline \multirow{2}{*}{ C2C-M } & $\mathrm{A}$ & 37 & 59 & 205 \\
\cline { 2 - 5 } & $\mathrm{B}$ & 38 & 59 & 219 \\
\hline \multirow{2}{*}{ C3C-T } & $\mathrm{A}$ & 22 & 47 & 170 \\
\cline { 2 - 5 } & $\mathrm{B}$ & 26 & 50 & 186 \\
\hline \multirow{2}{*}{ C4S1-L } & $\mathrm{A}$ & 6 & 6 & 19 \\
\cline { 2 - 5 } C5S1-M & $\mathrm{B}$ & 4 & 11 & 52 \\
\hline C6S2-M & $\mathrm{A}$ & 32 & 41 & 144 \\
\hline
\end{tabular}

\subsection{Performance Assessment based on Material Strains}

Performance based seismic design philosophy is based on the approach that ensures certain performance states for the construction of new bridges or for retrofitting existing bridges. The performance states such as Operational and Life Safety are usually defined qualitatively based on the damage state in the structure. However, the quantitative definition of these qualitative damage states and hence the performance limit needs to be defined with respect to an engineering deformation criterion i.e. strain, displacement and curvature. Material strain values such as concrete compressive strain and steel tensile strain are inherent material properties that can be related to the limit states and have been commonly used in the literature (Kowalsky, 2000). Past research has been dedicated for the development of strain-based limit states for reinforced concrete bridge columns, as shown in Table 3.21. As can be seen, in the two-level performance criteria required by the Oregon Department of Transportation for SDC D (ODOT, 2017), 
a structural performance is described with two discrete performance levels and two seismic hazards, herein Operational Performance Level for a full rupture Cascadia Subduction Zone Earthquake and Life Safety Performance Level for a 1000-year return period event. Then, each performance level is quantified in terms of concrete and steel strains, where $\varepsilon_{s}$ is the reinforcing steel strain, $\varepsilon_{\text {sh }}$ is the reinforcing steel strain at the onset of strain hardening, $\varepsilon^{\mathrm{R}}$ su is the reduced ultimate tensile strain in the reinforcing steel, $\varepsilon_{\mathrm{cc}}$ is the strain in the confined section of columns, and $\varepsilon_{\mathrm{cu}}$ is the ultimate concrete strain computed using Mander's model. From the table, it can be seen that the difference in concrete strain between the Operational and the Life Safety performance level is only 0.003 , and caused by the low concrete confinement, typical of RC columns built prior to 1970. Goodnight and Kowalsky (2015) experimentally evaluated the performance strain limit recommended by Kowalsky (2000) in a test matrix of 30 well-detailed RC bridge columns, and the results also presented in Table 3.21. Serviceability strain limits were maintained, while new strain limit expression for Life Safety were developed for the compressive strain at initial yielding of confinement steel and the tension strain preceding bar buckling. These two expressions we evaluated using the columns' properties of this study and are presented in Table 3.21. Nonetheless, the expressions proposed by Goodnight were obtained from well-detailed concrete columns detailed with spiral reinforcement and low pitch. Therefore, comparing these limits to ODOT's criteria, the difference in compressive strain between the Operational and Life Safety performance is greater. On the other hand, well agreement was found between the measured peak tensile 
strain preceding bar buckling and the new expression developed by the authors. The values reported by in Table 3.21 will be compared to the ones developed in this study.

Table 3.21 Quantitative limit state in terms of strains

\begin{tabular}{c|c|c|c}
\hline $\begin{array}{c}\text { Performance } \\
\text { Level }\end{array}$ & $\begin{array}{c}\text { Kowalski } \\
(2000)\end{array}$ & $\begin{array}{c}\text { Oregon Department of } \\
\text { Transportation } \\
(2017)\end{array}$ & $\begin{array}{c}\text { Goodnight et.al } \\
(2015)\end{array}$ \\
\hline $\begin{array}{c}\text { Operational } \\
\text { (Serviceability) }\end{array}$ & $\begin{array}{c}\varepsilon_{\mathrm{c}}=0.004 \\
\varepsilon_{\mathrm{s}}=0.015\end{array}$ & $\begin{array}{c}\varepsilon_{\mathrm{c}}=0.005 \\
\varepsilon_{\mathrm{s}}=2 \cdot \varepsilon_{\mathrm{sh}}=0.03\end{array}$ & $\begin{array}{c}\varepsilon_{\mathrm{c}}=0.004 \\
\varepsilon_{\mathrm{s}}=0.015\end{array}$ \\
\hline $\begin{array}{c}\text { Life Safety } \\
\text { (Damage Control) }\end{array}$ & $\begin{array}{c}\varepsilon_{\mathrm{cu}} \approx 0.018 \\
\varepsilon_{\mathrm{s}}=0.06\end{array}$ & $\begin{array}{c}\varepsilon_{\mathrm{cc}}=0.9 \cdot \varepsilon_{\mathrm{cu}} \approx 0.008 \\
\varepsilon_{\mathrm{s}}=\varepsilon_{\mathrm{su}}^{\mathrm{R}}=0.09\end{array}$ & $\begin{array}{c}\text { spiral } \\
\varepsilon_{\text {cyield }}=0.017 \\
\varepsilon_{\text {bark }}^{\text {barling }}\end{array}$ \\
\hline
\end{tabular}

In order to relate the global performance levels to component's levels, a five-level component performance is shown in Table 3.22. This table is based on the work carried out by Hose \& Seible (1999) and adapted to correspond with ODOT's performance criteria. The table also shows the corresponding performance criteria suggested by Hose \& Seible, in which the Operational level corresponds to first yielding and minor cracking, and Life Safety corresponds to the onset of spalling.

In the following subsections, each limit state is described for all the test specimens to determine if ground motion duration affects the point at which each limit state happened. For each of the five limit states, experimental longitudinal reinforcement tensile strain, concrete compressive strain, drift ratio, and ductility are presented in Table 3.23. The reported values are the average of each direction. It is worth mentioning that extreme concrete compressive strains of the columns were obtained using the results from the strains in the reinforcing steel and a linear strain profile for a circular section. In the 
case of spliced specimens, tensile strain measurements in the starter bars were used; meanwhile, both dowels and longitudinal reinforcement strain gauge data were used for the compression strains.

Table 3.22 Component performance levels adapted from Hose \& Seible (1999)

\begin{tabular}{c|c|c|c|c|c}
\hline Level & $\begin{array}{c}\text { Damage } \\
\text { Classification }\end{array}$ & $\begin{array}{c}\text { Component } \\
\text { Performance }\end{array}$ & $\begin{array}{c}\text { Repair } \\
\text { Description }\end{array}$ & $\begin{array}{c}\text { Hose \& } \\
\text { Seible } \\
\text { Global } \\
\text { Performance }\end{array}$ & $\begin{array}{c}\text { ODOT's } \\
\text { Performance } \\
\text { Levels }\end{array}$ \\
\hline I & No & Cracking & No Repair & $\begin{array}{c}\text { Fully } \\
\text { Operational }\end{array}$ & - \\
\hline II & Slight & First Yield & $\begin{array}{c}\text { Possible } \\
\text { Repair }\end{array}$ & Operational & - \\
\hline III & Moderate & $\begin{array}{c}\text { Onset of } \\
\text { Spalling }\end{array}$ & $\begin{array}{c}\text { Minimum } \\
\text { Repair }\end{array}$ & Life Safety & Operational \\
\hline IV & Extensive & $\begin{array}{c}\text { Complete } \\
\text { Spalling }\end{array}$ & Repair & $\begin{array}{c}\text { Near } \\
\text { Collapse }\end{array}$ & - \\
\hline V & $\begin{array}{c}\text { Local } \\
\text { Failure/Collapse }\end{array}$ & $\begin{array}{c}\text { Bar Buckling } \\
\text { Crushing }\end{array}$ & Replacement & Collapse & Life Safety \\
\hline
\end{tabular}

\subsubsection{Cracking}

This damage state was related to strains by computing the cracking moment, following the procedure described in ACI 318 (ACI, 2014). This cracking moment was compared to the moment at the column base. This methodology was used because it was not possible to visually identify the point at which each specimen developed the first crack. Visual inspection of residual cracking was done after each motion amplitude. Residual crack widths were different depending on the demand duration. Test specimens subjected to subduction ground shaking showed residual cracks widths about $6.3 \mathrm{~mm}$ (0.27 in.) to $7.9 \mathrm{~mm}(0.31 \mathrm{in}$.), whereas under crustal shaking residual cracks widths were 
about $3.4 \mathrm{~mm}(0.13 \mathrm{in}$.). First test series showed horizontal cracking pattern at the transverse reinforcement level. Second test series showed vertical cracks along the spliced bars.

\subsubsection{First Yield of Longitudinal Reinforcement}

This damage state was not feasible to be qualitatively described because the nature of test procedure. Conversely, the instrumentation allowed describing this limit state quantitatively. Concrete compressive strains values were similar among the specimens tested and ranged from -0.001 to -0.0016 . From Table 3.23 , it can be seen that the effects of subduction ground motion duration have no impact on the material strain describing the limit state of first yield. Following this limit state, stiffness degradation was exhibited for all specimens.

\subsubsection{Concrete Cover Spalling}

The initiation and extent of spalling are key parameters for performance assessment. The onset of spalling, related to the ODOT's Serviceability performance level, is a good indicator of local damage, and would require minimum repair (Hose \& Seible, 1999). The extent of spalling along the column height is also important, as it determines the minimum length over which a significant repair would be required.

Concrete compressive strain at the onset of spalling limit state were found to be higher in the specimens subjected to subduction records and ranged from -0.0064 to 0.0082, compare to -0.006 for the crustal ground motion. Same situation was found for the tensile strains in the reinforcing steel. Specimens under subduction records exhibited higher strains and ranged from 0.016 to 0.02 , whereas specimens under crustal motion 
had tensile strains 0.01 and 0.013 (C1C-L and C4S1-L, respectively). According to Table 3.21, ODOT cites a concrete compressive strain of -0.005, while Goodnight (2015) quotes a strain value of -0.004 . From the test results, all specimens exceeded the concrete compressive strain limits for the Serviceability Performance Level suggested by the authors. Also, from the table, Goodnight cites a tensile steel strain of 0.015 for the same performance level, where specimens under subduction records only exceeded it.

Visual inspection of the test specimens revealed that extent of the spalling region varies depending on the duration of the ground motion. The increased number of loading cycles imposed by subduction records was traduced in a larger spalled area, which may require a more extensive repair method. After the first ground motion amplitude, C2C-M exhibited significant spalling (length of the spalled region about one-half of the crosssection depth), whereas specimen C1C-L exhibited spalled region of one-eighth of the cross-section depth. For short lap-spliced specimens, sudden failure did not allow the extent of concrete spalling and exhibited severe core crushing. Specimen C6S2-M exhibited a spalled region length similar to C3C-T after motion amplitude A (about 130 $\mathrm{mm})$, but the damage extended to core concrete exposing the column reinforcement at the base. Tensile steel and concrete compressive strains were similar among the specimens that exhibited the complete spalling limit state (Table 3.23); however, these strains were reached at different ground motion amplitudes, i.e. specimens under subduction records exhibited the complete spalling in motion amplitude A, whereas $\mathrm{C} 1 \mathrm{C}-\mathrm{L}$ reached this limit in the second motion amplitude. 


\subsubsection{Bar Buckling and Core Crushing}

Level five of the component performance level adversely affect the seismic response and failure of the bridge column may be expected, leading to replacement of the structural component. Subduction ground motions limited the deformation capacity of nonspliced specimens by longitudinal bar buckling and followed by core crushing. Bar buckling occurred after reversal from peak tensile strain of 0.035 in $\mathrm{C} 2 \mathrm{C}-\mathrm{M}$ and 0.029 in C3C-T. From Table 3.23, it can be seen that for subduction records these values exceed the expression proposed by Goodnight for the Life Safety performance level, which ratify the hypothesis that more structural damage is expected from long duration earthquakes (Raghunandan \& Leil, 2013). Furthermore, results indicate that increased number of loading cycles imposed by long-duration subduction demands have a strong influence on bar buckling. This outcome is in agreed with previous research, which has shown that the

cyclic history has a strong influence on bar buckling (Kunnath, 1997), (Goodnight et al., 2013).

Core crushing limit state was defined for specimens that showed sudden lapsplice failure with extensive core damage. From Table 3.23, it can be observed that the strain limits for C4S1-L and C5S1-M are similar, however they occurred at different motion amplitudes. Although the limit strains for column C5S1-M does not exceed the expression presented in Table 3.23 during motion amplitude A for the Life Safety performance level, its loss of load-carrying capacity indicates possible replacement or more invasive repair techniques may be needed. 
Table 3.23 Summary of limit state strains for all specimens

\begin{tabular}{|c|c|c|c|c|c|c|c|}
\hline \multirow{2}{*}{$\begin{array}{c}\text { Limit } \\
\text { State } \\
\end{array}$} & \multirow[b]{2}{*}{ Measure } & \multicolumn{6}{|c|}{ Specimen } \\
\hline & & C1C-L & C2C-M & $\mathrm{C} 3 \mathrm{C}-\mathrm{T}$ & C4S1-L & C5S1-M & C6S2-M \\
\hline \multirow{4}{*}{ Cracking } & $\varepsilon_{\mathrm{s}}$ & 0.00014 & 0.00013 & 0.0002 & - & - & 0.00013 \\
\hline & $\varepsilon_{\mathrm{c}}$ & 0.0002 & 0.0002 & 0.0002 & - & - & 0.0002 \\
\hline & Drift (\%) & 0.09 & 0.10 & 0.14 & - & - & 0.11 \\
\hline & $\mu$ & 0.1 & 0.1 & 0.1 & - & - & 0.1 \\
\hline \multirow{4}{*}{$\begin{array}{l}\text { First } \\
\text { Yield }\end{array}$} & $\varepsilon_{\mathrm{s}}$ & 0.0018 & 0.0018 & 0.0018 & 0.0018 & 0.0018 & 0.0018 \\
\hline & $\varepsilon_{\mathrm{c}}$ & 0.0010 & 0.0011 & 0.0011 & 0.0010 & 0.0012 & 0.0016 \\
\hline & Drift (\%) & 0.55 & 0.56 & 0.81 & 0.61 & 0.50 & 0.69 \\
\hline & $\mu$ & 0.6 & 0.6 & 0.8 & 0.5 & 0.4 & 0.7 \\
\hline \multirow{4}{*}{$\begin{array}{l}\text { Onset of } \\
\text { Spalling }\end{array}$} & $\varepsilon_{\mathrm{s}}$ & 0.01 & 0.016 & 0.015 & 0.013 & 0.017 & 0.02 \\
\hline & $\varepsilon_{\mathrm{c}}$ & 0.0059 & 0.0071 & 0.0082 & 0.0060 & 0.0064 & 0.0065 \\
\hline & Drift (\%) & 3.08 & 2.76 & 3.17 & 2.97 & 2.87 & 2.63 \\
\hline & $\mu$ & 3.5 & 3.1 & 3.2 & 2.2 & 2.2 & 2.7 \\
\hline \multirow{4}{*}{$\begin{array}{l}\text { Complete } \\
\text { Spalling }\end{array}$} & $\varepsilon_{\mathrm{s}}$ & 0.024 & 0.026 & 0.024 & - & - & 0.029 \\
\hline & $\varepsilon_{\mathrm{cc}}$ & 0.0096 & 0.0097 & 0.0096 & - & - & 0.0098 \\
\hline & Drift (\%) & 4.33 & 3.59 & 3.74 & - & - & 3.65 \\
\hline & $\mu$ & 4.9 & 4.1 & 3.8 & - & - & 3.8 \\
\hline \multirow{4}{*}{$\begin{array}{c}\text { Bar } \\
\text { Buckling / } \\
\text { Core } \\
\text { Crushing }\end{array}$} & $\varepsilon_{\mathrm{s}}$ & - & 0.035 & 0.029 & 0.026 & 0.027 & - \\
\hline & $\varepsilon_{\mathrm{cc}}$ & - & 0.013 & 0.011 & 0.011 & 0.010 & - \\
\hline & Drift (\%) & - & 4.28 & 3.36 & 4.12 & 3.42 & - \\
\hline & $\mu$ & - & 4.8 & 3.4 & 3.2 & 2.6 & - \\
\hline
\end{tabular}

Finally, correlation between damage levels and engineering demand parameters is presented in Table 3.24. Chosen engineering demand parameter, i.e., steel and concrete strains, will be used in the Chapter 5. The values listed represent the average of the values presented in Table 3.23 for the tested substandard reinforced concrete columns. 
Table 3.24 Substandard RC bridge column performance parameters (Limit States)

\begin{tabular}{c|c|c|c|c}
\hline Level & $\begin{array}{c}\text { Performance Level } \\
\text { (this study) }\end{array}$ & Limit State & $\varepsilon_{\mathrm{s}}$ & $\varepsilon_{\mathrm{c}}\left(\varepsilon_{\mathrm{cc}}{ }^{1}\right)$ \\
\hline I & - & Cracking & 0.00015 & 0.0002 \\
\hline II & Fully Operational & First Yield & 0.0018 & 0.0012 \\
\hline III & Operational & $\begin{array}{c}\text { Onset of } \\
\text { Spalling }\end{array}$ & 0.016 & 0.006 \\
\hline IV & Life Safety & $\begin{array}{c}\text { Complete } \\
\text { Spalling }\end{array}$ & 0.026 & $0.010^{1}$ \\
\hline V & Near Collapse & $\begin{array}{c}\text { Bar Buckling/ } \\
\text { Core Crushing }\end{array}$ & 0.032 & $0.012^{1}$ \\
\hline
\end{tabular}

${ }^{1} \varepsilon_{c c}$ : concrete compressive strain at the column core 


\section{CHAPTER 4. Numerical Investigation of the Effects of Subduction Earthquakes on the Seismic Performance of Substandard RC Bridge Columns}

\subsection{Introduction}

Numerical models calibrated against experimental results are crucial for predicting the seismic performance of structures when subjected to strong ground motions, especially subduction zone ground motions which are the focus of this study. In this sense, this chapter focuses on the nonlinear modeling of substandard RC bridge columns. The results of the numerical modeling were compared to the shake table experimental results described in Chapter 3. The OpenSees software (Open System for Earthquake Engineering Simulation) was utilized for modeling and analyzing the response of the tested specimens by performing nonlinear dynamic analyses. OpenSees is an open-source software framework for developing applications to simulate the performance of structural and geotechnical systems subjected to earthquakes created at the Pacific Earthquake Engineering Research Center (OpenSees, 2013). This software possesses advanced capabilities for modeling and analyzing the linear and nonlinear behavior of structural systems using a variety of constitutive material models, elements and solution algorithms. Additionally, the performance assessment of a full-scale single reinforced concrete bridge column when subjected to subduction zone ground motions is investigated by performing nonlinear time history analyses. 


\subsection{Numerical Model of Large-Scale Bridge Columns}

In this section, a numerical model is evaluated to characterize the behavior of the large-scale RC bridge columns that were part of the experimental program presented in Chapter 3. The large-scale bridge columns specimens consisted of a circular cross-section of 18 in. diameter fixed connected to a foundation block, and a height of 96 in. Even though some specimen differed in the detailing used in the column design, i.e., location and length of the lap-spliced longitudinal starter bars in the footing, one numerical model was considered with some minor modifications to account for the differences in the damage observed. OpenSees (2013) was utilized for numerical simulations.

The large-scale bridge columns used in the experimental program were modeled using a distributed-plasticity approach as described in Chapter 2. In this approach, the RC column is modeled using a force-based beam-column element with distributed plasticity where yielding is allowed at any integration point along the element length (Neuenhofer \& Filippou, 1997). The total length of the element was divided into five integration points. Gauss-Lobatto integration method was used to evaluate the element response at those integration points. The cross section of the column was represented by a finite number of fibers representing the uniaxial characteristics of the concrete cover (unconfined), concrete core (confined) and reinforcing steel. A radial discretization scheme was used to discretize the cross-section, and it was as follow: 16 radial core divisions, 18 transverse core divisions, 2 radial unconfined cover division, and 18 transverse cover division. The core concrete, cover concrete, and longitudinal steel fibers each have a uniaxial stress-strain model associated with them. The Concrete 04 with 
degraded linear unloading/realoading stiffness and tensile strength with exponential decay was used in this study to model both unconfined and confined concrete. The unconfined concrete properties were based on the test day concrete strength for each specimen (Table 3.1). The confined concrete properties were calculated according to Mander et al. (1988).

The reinforcing steel was modeled using the Hysteretic uniaxial material given its capabilities for capturing the pinching of force and deformation observed in column with lap splices. Moreover, this model is capable of reproducing the strength and stiffness degradation due to concrete crushing, bar buckling and bar fracture. The parameters required to define the material model include: three points for the tri-linear stress-strain envelope, and four optional values to include pinching and damage in the hysteresis model. The stress-strain values for the reinforcing steel used in the numerical model represent the measured values from the experimental study and were obtained from tensile testing of a coupon (Table 3.1). The parameters pinchx and pinchy were set equal to 1.0 in order to represent the observed pinching behavior of the columns. To account for the differences in the final damage state between specimens with short lap splice at the column base and the specimens without lap splice or a relatively long but still inadequate lap splice at the base, the damage parameters of this model were adjusted based on the experimental results. The parameter damage1, which accounts for the damage due to ductility, was set equal to 0.008 for the specimens with short lap splice at the column base (i.e., C4S1-L and C5S1-M) and 0.003 for the other specimens. The parameter damage2, which accounts for the damage due to energy was set equal to 0.005 
for the specimens with short lap splice and 0.002 for the other specimens. The degraded unloading stiffness parameter based on ductility was set equal to 0.3 for all specimens.

As it is known, the lateral deformation of a reinforced concrete column is the sum of the displacement components due to bending, slip and shear. Flexural behavior is accurately captured by the fiber-based model, but it does not include the shear and slip components. Shear deformations were introduced into the model through the use of the section aggregator command, in which a constant shear modulus equal to $0.2 E_{\mathcal{C}}$ was used following the recommendations of Elwood and Eberhard (2009). To account for the flexibility in the lateral response of the specimens due to bond slip, a zero-length section following the model proposed by Ghannoum (2007) and the recommendations of Mehary et al. (2018) were used. In this bond-slip model, the zero-length fiber section is modeled using an equivalent stress-slip relationship for the concrete and steel fibers, as described in Chapter 2. It is worth noting that the concrete and steel fibers in the zero-length element had the same fiber discretization scheme used in the force-based beam-column element. The slip displacement at yield ( $\mathrm{sy})$ in the steel fibers were obtained from the measurements of the LVDTs located at the base of the columns. Values of $0.032 \mathrm{in}$, 0.029 in., 0.045 in., 0.060 in., 0.067 in., and 0.039 in. for the slip displacement at yield was used in the distributed-plasticity model for C1C-L, C2C-M, C3C-T, C4S1-L, C5S1M, and C6S2-M respectively. The Steel02 Giuffre-Menegotto-Pinto model with isotropic hardening was used to model the steel fibers in the bond-slip zero-length element because its easy implementation. Uniaxial concrete material model Concrete 04 was used for the confined and unconfined concrete in the bond-slip zero-length element. 
To avoid discontinuities in the steel stresses and neutral axis location between the fibers of the nonlinear beam/column element and the bond-slip section (Ghannoum, 2007), the concrete strains were modified by a scale factor, $\mathrm{SF}_{\mathrm{conc}}=\mathrm{sy}_{\mathrm{y}} / \varepsilon_{\mathrm{y}}$. Even though this scale factor is dimensionally incorrect, it allows an increase in the concrete strains to maintain compatibility between the fiber sections. Scale factors of 18, 17, 25, 34, 38, and 22 were employed for C1C-L, C2C-M, C3C-T, C4S1-L, C5S1-M, and C6S2-M respectively.

Even though the Hysteretic material model is able to reproduce the damage in the specimen due to ductility and energy, it does not account for the failure of reinforcing bars due to low-cycle fatigue. To account for this effect, the fatigue material developed in OpenSees was wrapped to the parent Hysteretic material assigned to the reinforcing bars and discussed previously. The fatigue material quantifies the accumulated damage in the reinforcing bars due to low-cycle fatigue using a modified rainflow cycle counter to track strain amplitudes. The cycle counter is used in concert with Miner's rule (Miner, 1945) and based on Coffin-Manson low-cycle fatigue relationships ( (Manson \& Hirschberg, 1963), and (Coffin, 1962)). Once the fatigue material model reaches a damage level of 1.0 , the force (or stress) of the parent material (Hysteretic) becomes zero. Two parameters are required to define this material model: $\mathrm{m}$, which represents the slope of Coffin-Manson curve in log-log space, and $\varepsilon o$, which represent the value of strain at which one cycle will cause failure. The parameter $m$ was set equal to -0.458 for all specimens. Parameter $\varepsilon O$ was set equal to 0.191 for C4S1-L and C5S1-M, and 0.125 for the rest of the specimens. 
Nonlinear time history analysis was conducted in OpenSees using the same numerical model for the specimen and the IMS described in Chapter 2. An illustration of the OpenSees model is depicted in Figure 2.11. In order to replicate the measured behavior of the specimens, the acceleration histories recorded by the shake table at each motion amplitude of the test protocol were used as the input ground motion. To account for the progressive damage observed in the experiments, the recorded acceleration histories at each motion amplitude were concatenated into a single record. Similarly, the recorded history of compressive axial load was applied at the top of the nonlinear beamcolumn element model. Damping characteristics in OpenSees are modeled using linear viscous damping following the Rayleigh model, in which the damping matrix at each time step is calculated as the linear combination of the mass $(M)$ and stiffness $(K)$ matrices. According to Charney (2008), the damping matrix for nonlinear analysis can be selected in three different ways: stiffness-only proportional, mass-only proportional and mass and stiffness proportional. Several studies have shown that for nonlinear time history analysis, the simulation of the viscous damping as the linear combination of the mass and initial stiffness matrices following Rayleigh method, results in underestimations of the structure displacements at high levels of deformation, when the hysteretic energy dissipation is low ((Sakai \& Unjoh, 2006); (Petrini et al., 2008); (Charney, 2008)). Instead, tangent stiffness-proportional viscous damping appears to be more appropriate for the nonlinear time history analysis and results in increased displacements at high ductility demands. Accordingly, a further refinement in the computational model was made by using a tangent stiffness-only proportional viscous damping with the damping 
coefficient indicated in Chapter 3 for the first natural period of each specimen. Results of the OpenSees simulation in terms of force-displacement hysteresis curves, displacement history and longitudinal steel strains are presented in the following for each specimen.

\subsubsection{Results for Specimens with Continuous Reinforcement}

The numerical model was calibrated against the results from the experimental shake table tests of RC bridge column specimens. The parameter used to resemble the seismic performance of the specimens with continuous reinforcement at the hinge zone were discussed in the previous section. As it was mentioned before, the measured material properties and the accumulated histories of acceleration recorded by the shake table were used as the input ground motions. The history of compressive axial load recorded by load cells on top of the column during the test was applied to the model specimen. Figure 4.1 illustrate the measured and calculated seismic performance of specimens $\mathrm{C} 1 \mathrm{C}-\mathrm{L}$ and $\mathrm{C} 2 \mathrm{C}-\mathrm{M}$ in terms of force-displacement hysteresis for each of the ground motion amplitudes used in the experimental program. Comparisons for Column $\mathrm{C} 3 \mathrm{C}-\mathrm{T}$ as well as measured and calculated seismic performance in terms of displacement histories for each motion amplitude are presented in the Appendix. From the results, it is noted that in general the numerical model replicated the stiffness, strength and displacement characteristics of the tested specimens reasonably well for all the ground motion amplitudes considered in the experimental campaign. Since low-cycle fatigue in the reinforcing bars is a major part of this model, strain histories were also compared (Figure 4.2 through Figure 4.4). The bar strains are slightly higher in the beginning of the 
first motion amplitude from the model than the experimental results, however they closely match the test values after the first peak tensile strain.
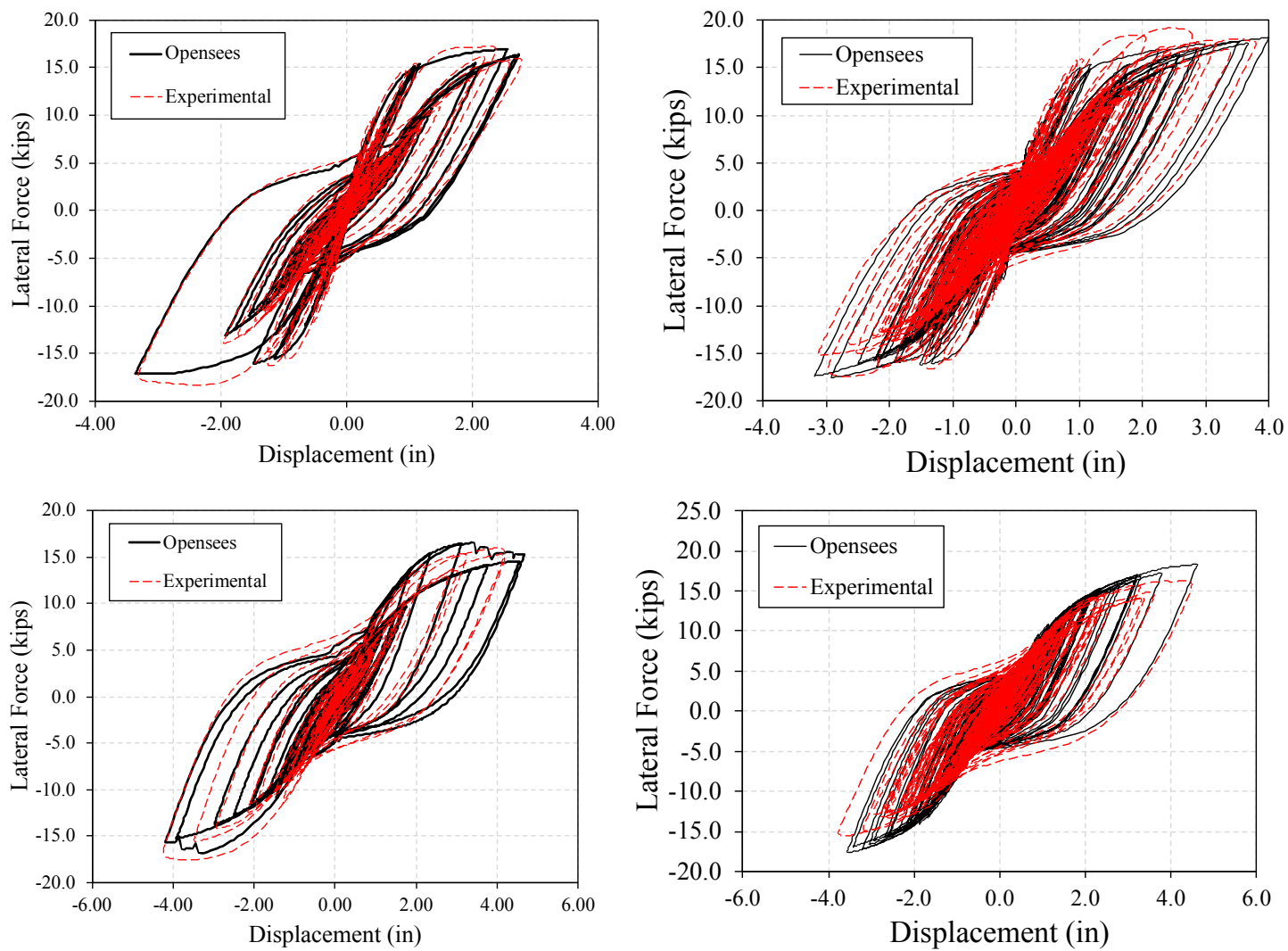

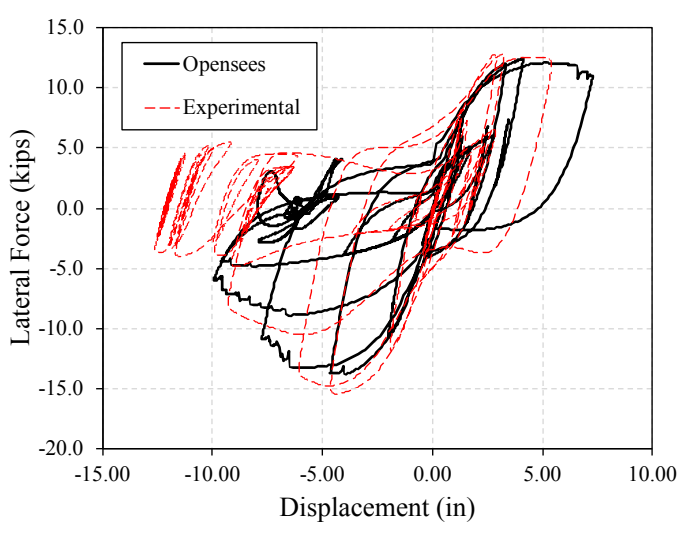

a)

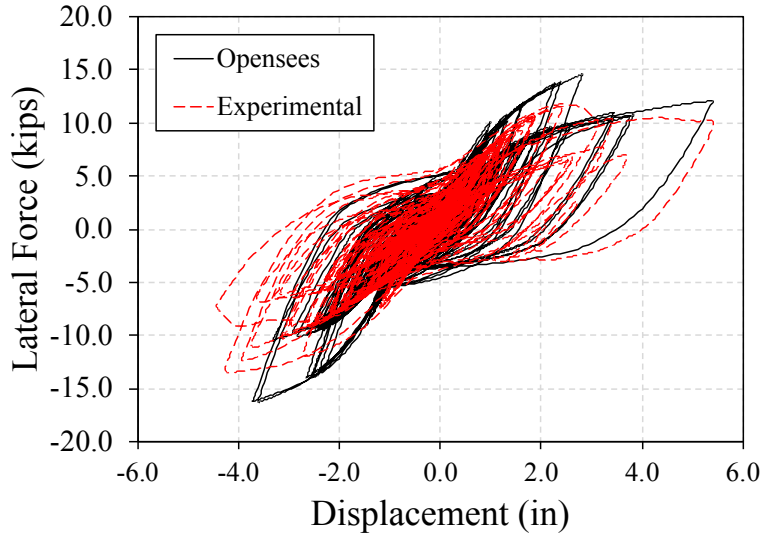

b)

Figure 4.1 Hysteresis curves comparison for all motion amplitudes. a) C1C-L, b) C2C-M 


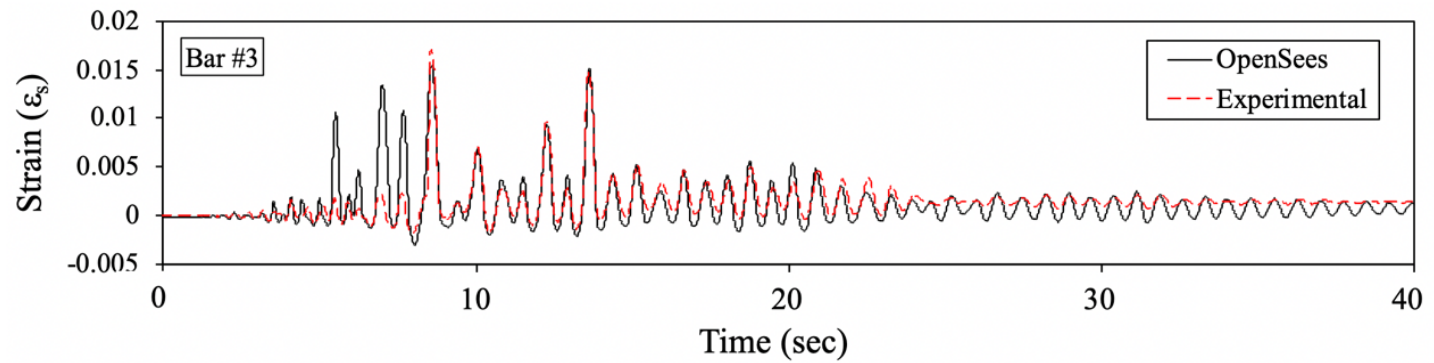

a)

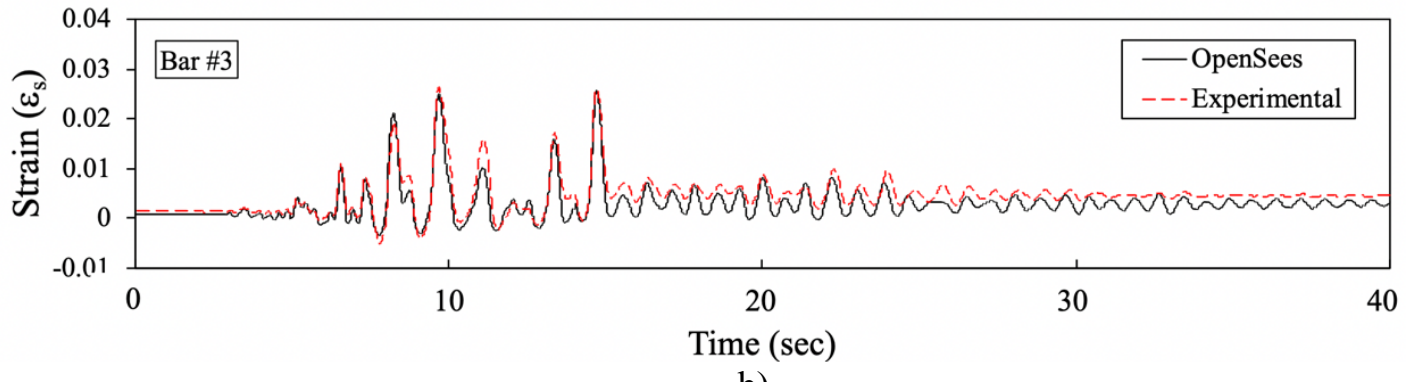

b)

Figure 4.2 Steel strain history comparison for Column C1C-L - Bar \#3. a) Amp.A, b) Amp.B

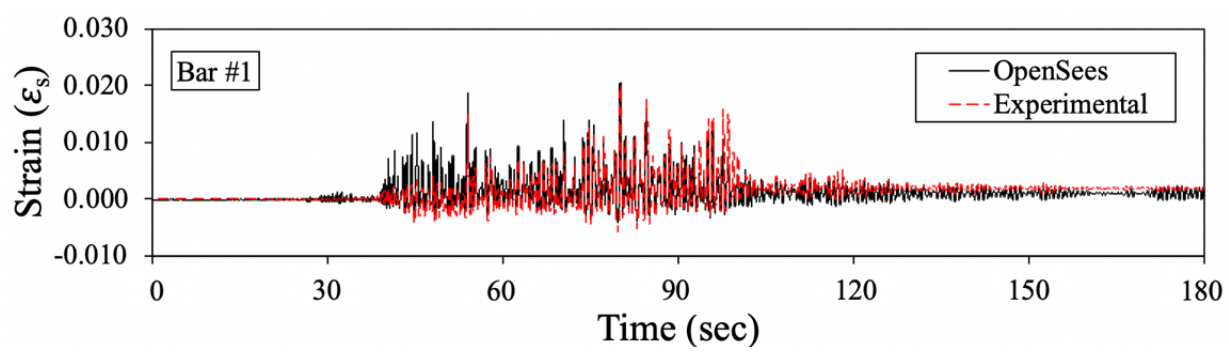

a)

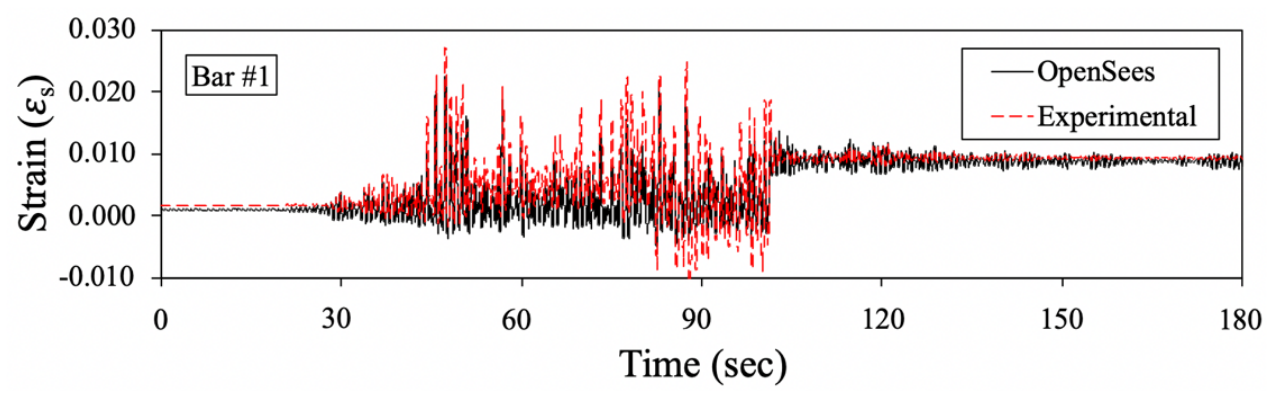

b)

Figure 4.3 Steel strain history comparison for Column C2C-L - Bar \#1. a) Amp.A, b) Amp.B 


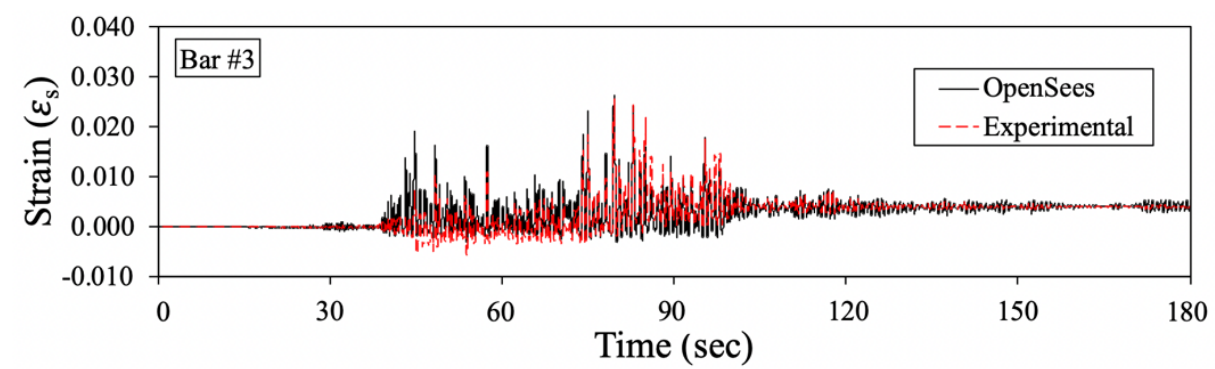

a)

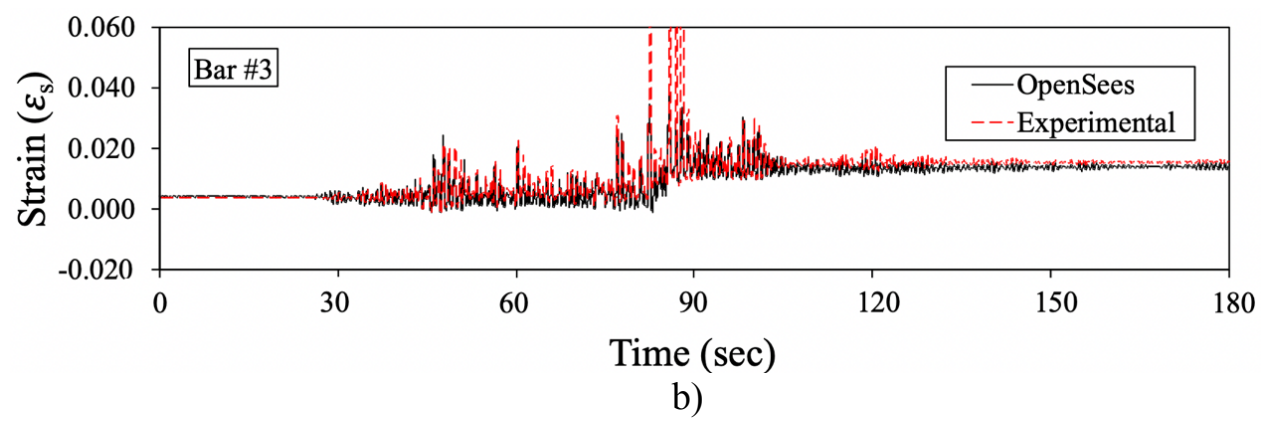

Figure 4.4 Steel strain history comparison for Column C2C-L - Bar \#3. a) Amp.A, b) Amp.B

\subsubsection{Results for Specimens with Lap Splice at Hinge Zone}

The parameter used to resemble the seismic performance of the specimens with lap splice at the hinge zone were discussed in the previous section. As it was mentioned before, the measured material properties and the accumulated histories of acceleration recorded by the shake table were used as the input ground motions. The history of compressive axial load recorded by load cells on top of the column during the test was applied to the model specimen. Figure 4.5 illustrate the measured and calculated seismic performance of specimens C4S1-L, C5S1-M, and C6S2-M in terms of forcedisplacement hysteresis for each of the ground motion amplitudes used in the experimental program. Measured and calculated seismic performance in terms of displacement histories for each motion amplitude are presented in the Appendix. It is worth mentioning that the calibration parameter obtained for specimens with continuous 
reinforcement at the hinge zone were used for Column C6S2-M, in order to reflect the observed performance during testing. From the results, it is noted that in general the numerical model replicated the stiffness, strength and displacement characteristics of the tested specimens reasonably well for all the ground motion amplitudes considered in the experimental campaign. Although there are differences between the measured and calculated response for specimen $\mathrm{C} 5 \mathrm{~S} 1 \mathrm{-M}$, the model was able to the strength deterioration once the lap-splice failure occurred. Even though low-cycle fatigue in the reinforcing bars is a major part of this model, loss of strain gauges during the lap splice failure in specimens C4S1-L. and C5S1-M hindered comparison between measured and calculated strain histories. However, prior to bond failure there was a good match between the experimental and simulated strain histories (Figure 4.6 through Figure 4.8). Similarly, good correlation was found between the measured and calculated strain histories for specimen $\mathrm{C} 6 \mathrm{~S} 2-\mathrm{M}$, which was provided of longer but still inadequate splice length at the hinge zone.

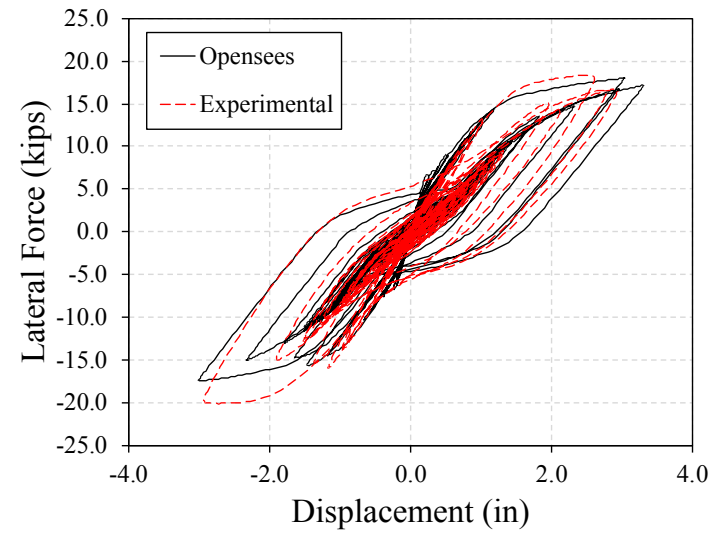

a)

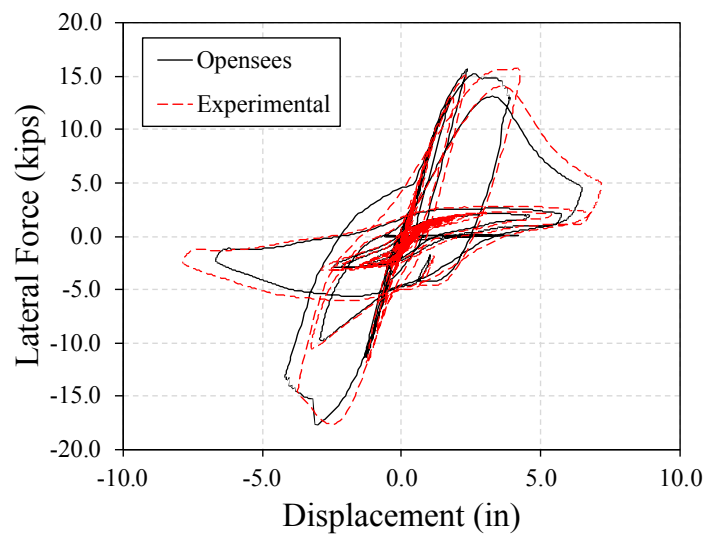

b) 


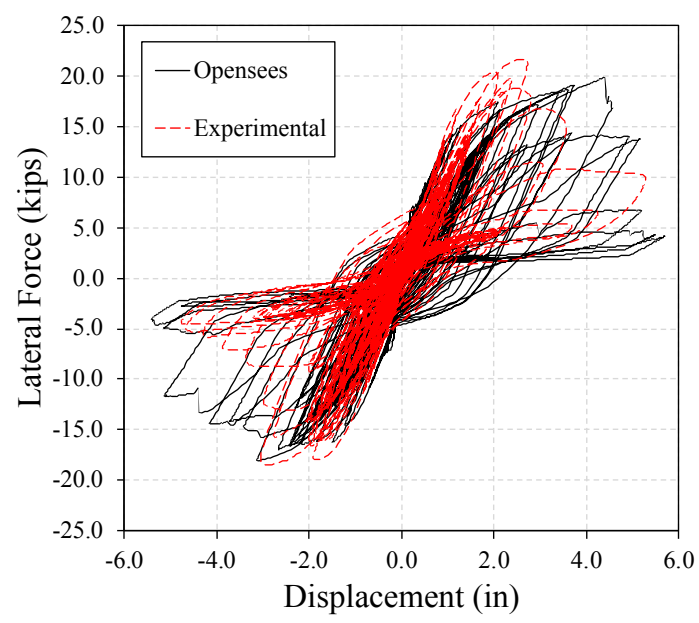

c)

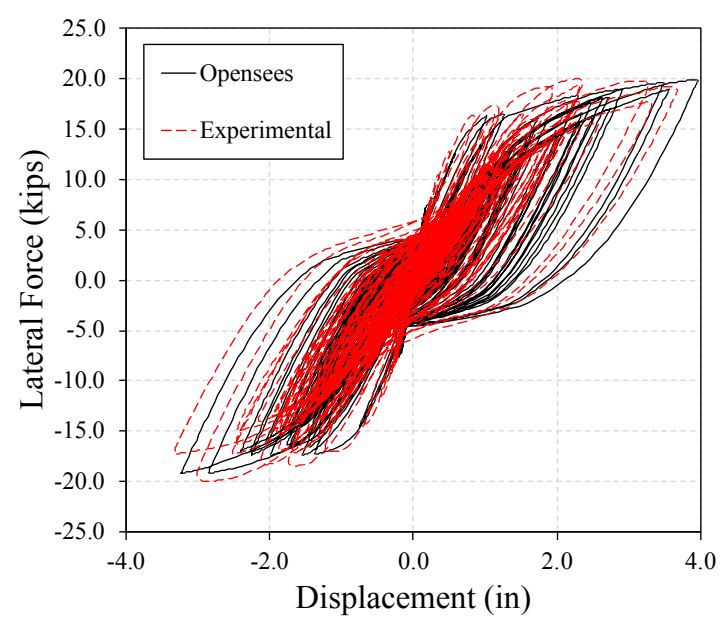

d)

Figure 4.5 Hysteresis curves comparison for lap-spliced specimen. a) C4S1-L during Amp.A, b) C4S1-L during Amp.B, c) C5S1-M during Amp.A, d) C6S2-M during Amp.A

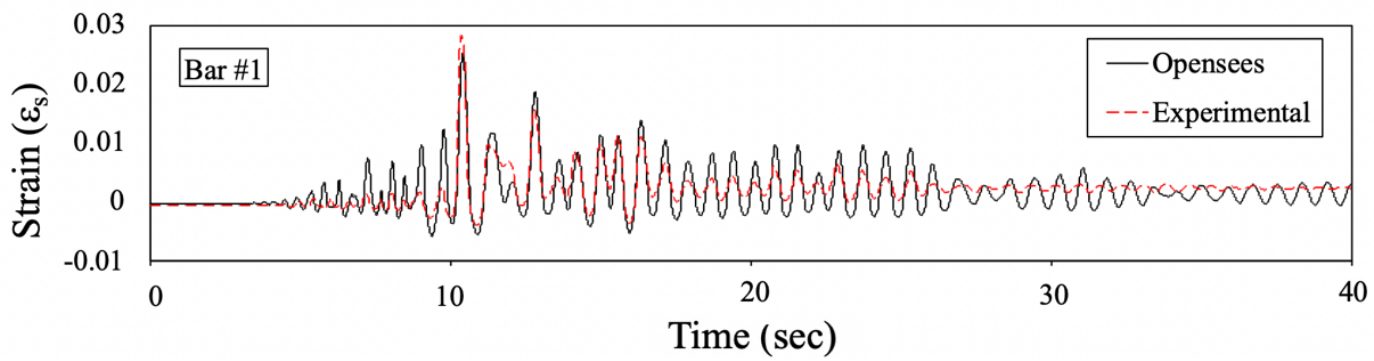

a)

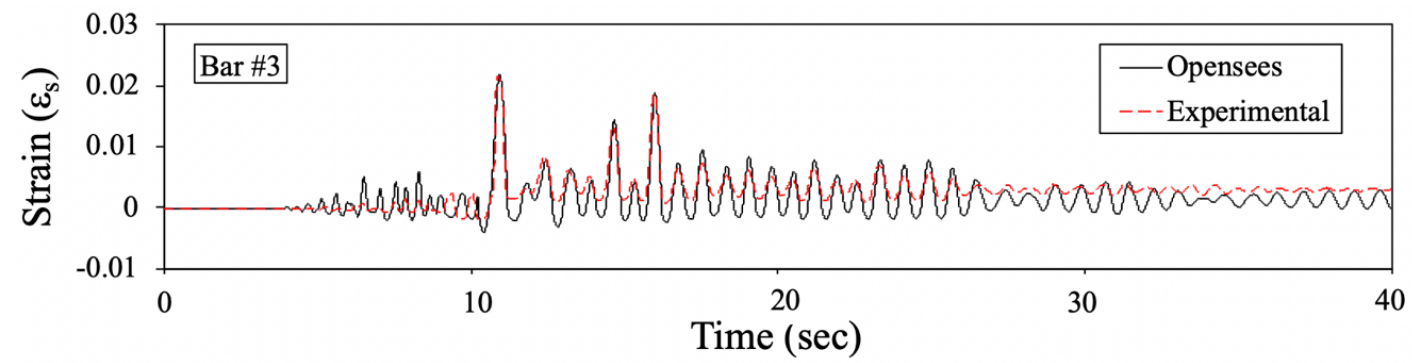

b)

Figure 4.6 Steel strain history comparison for Column C4S1-L in Amp.A. a) Bar \#1, b) Bar \#3 


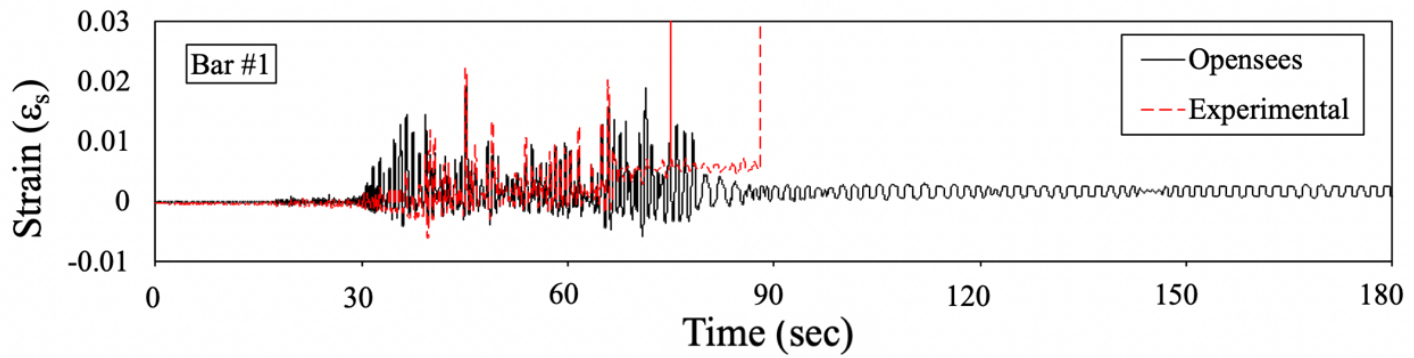

a)

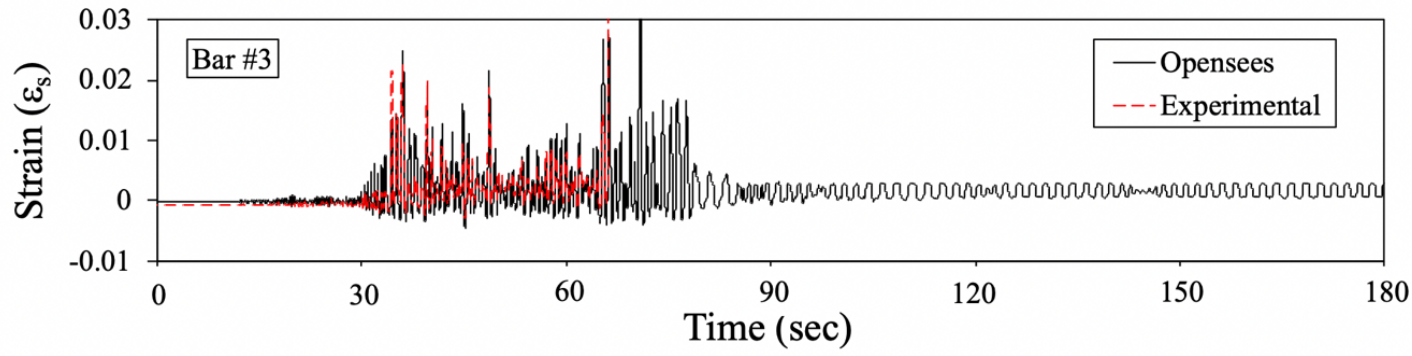

b)

Figure 4.7 Steel strain history comparison for Column C5S1-M in Amp.A. a) Bar \#1, b) Bar \#3

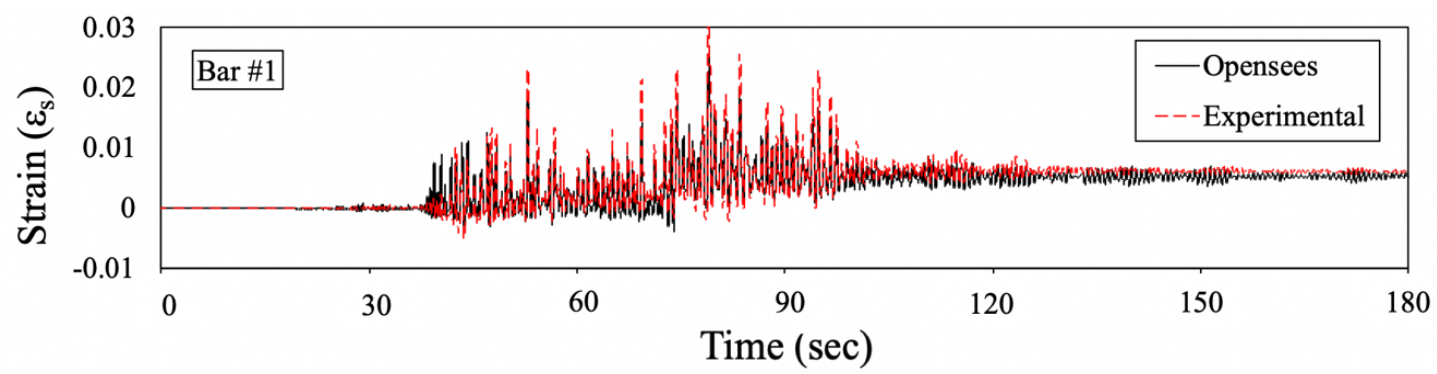

a)

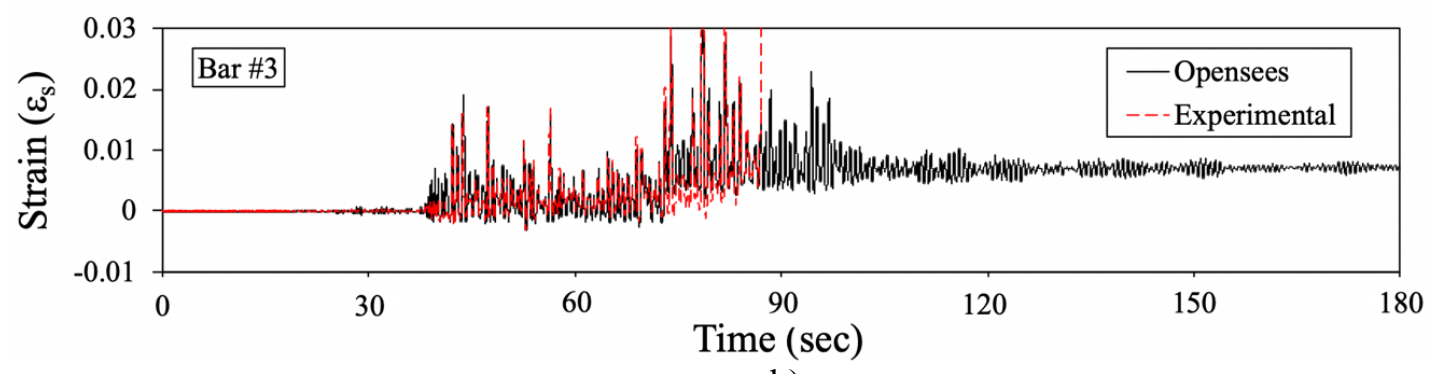

b)

Figure 4.8 Steel strain history comparison for Column C6S2-M in Amp.A. a) Bar \#1, b) Bar \#3 
The good match between the calibrated model and experimental results gave confidence for utilizing the calibrated parameters in the nonlinear dynamic analysis of a full-scale single bridge column, which is presented in the next section.

\subsection{Numerical Study of a Full-Scale Single RC Bridge Column}

According to modern seismic design philosophies, bridges under severe earthquake demands are designed to undergo substantial amounts of ductile inelastic deformation while sustaining their vertical loads without collapse. Concrete superstructures are usually designed to remain elastic while the inelastic deformations are accommodated in the substructure through the formation of plastic hinges. However, in RC bridges constructed in 1950 to mid-1970 in the Pacific Northwest, the bridge substructure was designed and built with minimum seismic considerations. This resulted in inadequate transverse reinforcement and confinement, no seismic detailing, and presence of lap-splices within plastic hinge zones. Since most of the bridges of that era are still in operation, and given that a megathrust earthquake is impending in the Pacific Northwest, the structural performance of these bridges should be well understood during the earthquake loading. To this end, computer simulations and numerical modeling have become crucial tools that enable design engineers and researchers to estimate system response performance.

In this section, the seismic performance of a full-scale model of the specimen part of the experimental program presented in Chapter 3 is studied through nonlinear time history analysis. The refined numerical model presented in the previous section was used to perform the nonlinear analyses. As it was explained in Chapter 3, the selection of the 
column model used in the experimental campaign was based on the $1 / 2$-scale model tested by Bazaez (2017) and laboratory constraints, and may represent an RC bridge column part of a single or multi-column bridge bent constructed in the 1950 to 1970 in the State of Oregon, USA. Boundary conditions for the full-scale column, fixed at the base and free at the top, may corresponded to a single-column bent subjected to ground excitation in the transverse direction of the bridge deck.

From the representative RC bridge assessed by Bazaez (2017) and depicted in Figure 3.1, column diameter corresponded to 3-ft. A longitudinal reinforcement ratio, $\rho_{l}$ of $1.2 \%$, which is just above the minimum required by AASHTO $\left(\rho_{l}>1 \%\right)$ (AASHTO, 2009), and consisted of eight, Grade 40 \#11 bars concentrically spaced around the column in a single layer. A transverse reinforcement ratio of $\rho_{t}$ of $0.2 \%$, which does not meet current requirements $\left(\rho_{t}>0.5 \%\right)$, represent the same reinforcement ratios found in the representative bridge bent (Figure 3.1) and corresponds to \#4 hoops, spaced at 12 in. on center. Moreover, from Figure 3.1, lap splices of $40 \mathrm{~d}_{\mathrm{b}}$ can be found at the base of columns and no seismic detailing was specified. The specified material properties for the representative bridge were $3.3 \mathrm{ksi}$ as compressive strength of concrete at 28 days and Grade 40 steel. To keep consistency with the experimental program, an axial load ratio of $0.01 \mathrm{~A}_{\mathrm{g}} \mathrm{f}_{\mathrm{c}}$ was used.

An illustration of the numerical model considered in this part of the study is depicted in Figure 2.14. In this model, the mass of the superstructure is lumped at the top of the column model. Given the presence of a relatively long but still inadequate lap splice at the column base, the calibrated parameters of C6S2-L were used for the 
numerical model. Fixed based support was assumed at the base of the column.

Fundamental period of $0.56 \mathrm{sec}$ was computed for the full-scale column. Three unscaled ground motions were used to evaluate the performance of the full-scale RC bridge column, and corresponded to the ones used in the experimental program as input for the shake table. The ground motions were selected from crustal and subduction sources, and are listed in Table 3.2. The good match between the calibrated model and experimental results gave confidence for utilizing the parameters in the nonlinear dynamic analysis of the full-scale column.

The results of the nonlinear analysis in terms of force-displacement hysteresis curves and steel strains are shown in Figure 4.9 and Figure 4.10, respectively. As it was observed in the experimental program, the full-scale column did not exhibit clear strength deterioration as well as hysteretic pinching, despite its detailing deficiencies. This can be attributed to the relatively long lap-splice length at the column base which allows the engagement and stress transfer between the starter bars and column reinforcement. Furthermore, it can be seen that the subduction ground motions, namely, Curico and Iwaki, subjected the full-scale column to many more inelastic cycles of small amplitude as compared to the crustal ground motion. This agrees with the results of the experimental program. 


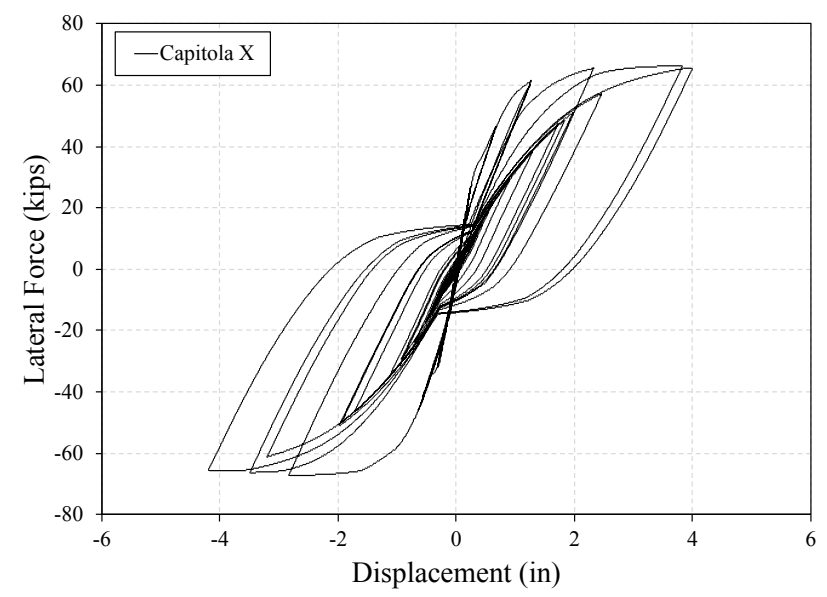

a)

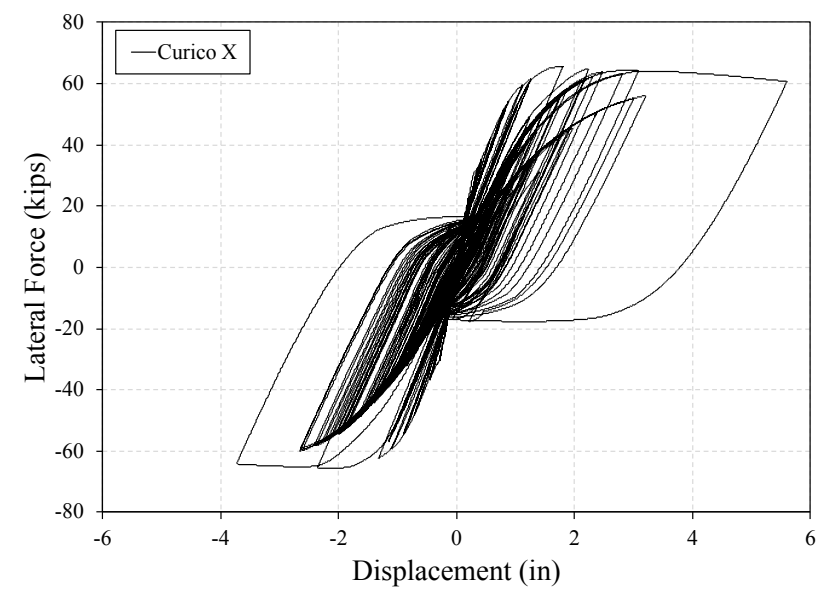

b)

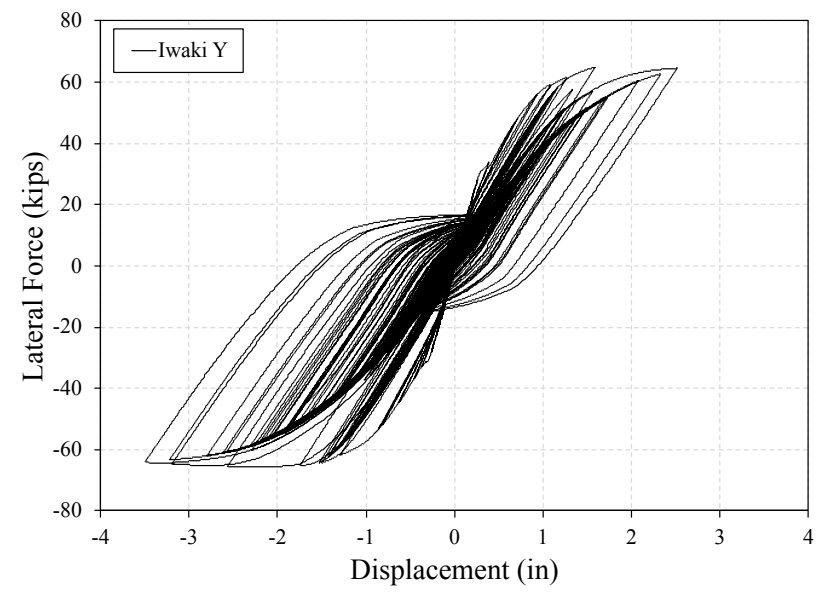

c)

Figure 4.9 Results of NLTHA for the full-scale single bridge column 
A comparison with the two-level performance criteria required by the state of Oregon, which is based on strain limitations for concrete and steel, is also depicted in Figure 4.10. This figure demonstrates that for subduction zone earthquake demands Operational performance level of response can be achieved for ground motions targeting the 1000-year event. Even though the Life Safety performance level of response was not achieved for any of the unscaled demands considered in this part of the study, the high value of tensile steel strain reported by ODOT (2017) (Table 3.23), may need to be revised based on the experimental and numerical results, since it does not represent the occurrence of bar buckling prior to the rupture of the rebar. This observation is addressed in the Chapter 5.

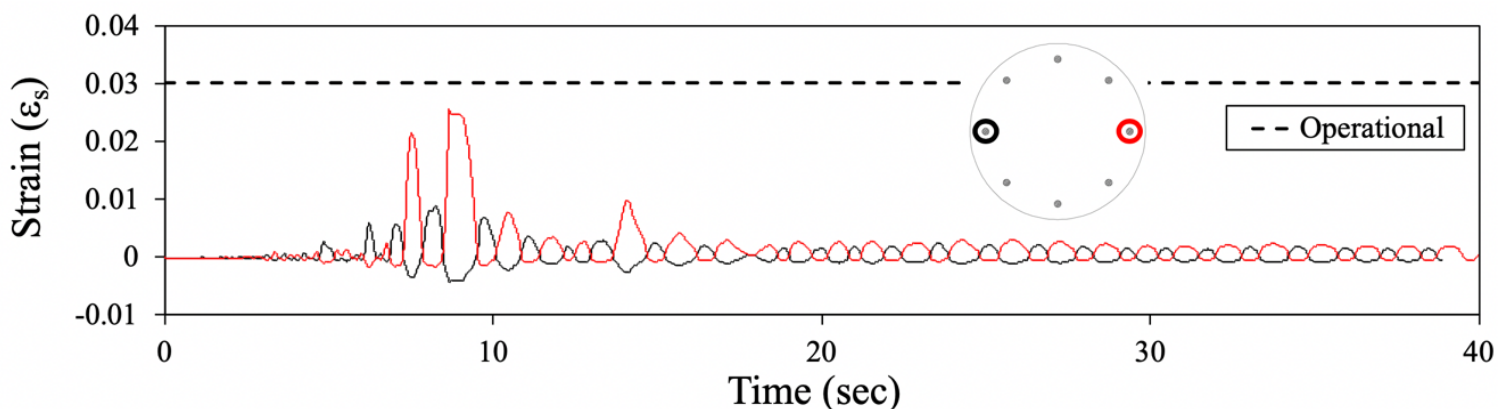

a)

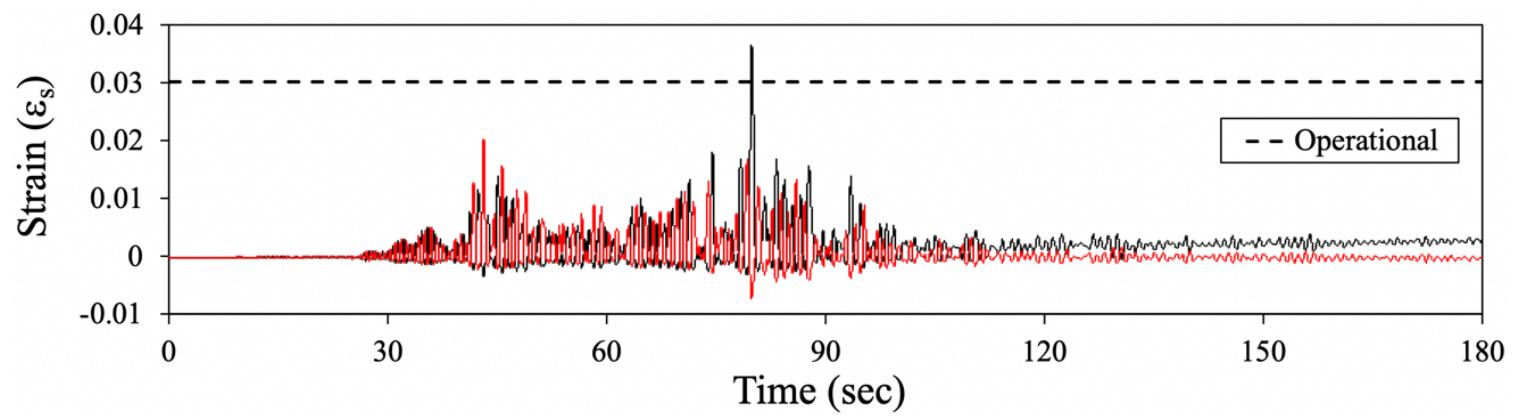

b)

Figure 4.10 Steel strain histories for the full-scale single bridge column 


\section{CHAPTER 5. Preliminary Recommendations of Performance Limits States for RC Bridge Columns Including the Effects of Subduction Earthquakes}

\subsection{Introduction}

Past earthquakes that have occurred in seismically active areas, show that bridge structures are one of the most vulnerable and expensive components of highway transportation systems. Despite of the potential high damage of subduction-zone ground motions, current seismic design provisions, performance assessments, and retrofit strategies do not explicitly consider the effects of this type of earthquakes. Their effects are not captured by the single acceleration design value used by modern seismic design codes. This is because megathrust earthquakes are not frequent compared to crustal earthquakes. Therefore, available recorded crustal ground motion dominate the database used for current seismic design and analytical studies. However, the occurrence of recently devastating seismic events in Chile and Japan, have increased the quantity and availability of long duration, large magnitude subduction zone ground motions to better study this subject.

Current seismic design standards are moving towards a performance-based design methodology, where a reinforced concrete bridge is designed to meet specified performance objectives which is a pair of structural performance level and a seismic demand level. Then, performance levels describe key damage states that need to be quantified through engineering limit states, such as material strain, displacement 
ductility, among others. Under this scenario, it becomes necessary to include the effects of subduction zone earthquakes in the evaluation and design process of reinforced concrete bridges. For this purpose, this chapter aims to investigate the effects of subduction zone earthquake in the seismic performance of substandard RC bridge columns by performing seismic fragility analysis. The OpenSees model, calibrated against the results of the experimental campaign presented in Chapter 3, is used for this purpose. Incremental dynamic analysis (IDA) is employed using ground motion from subduction and crustal sources. IDA is subjecting the model to a ground motion with a specific intensity, and the history and the peak material strain responses of the column are measured. The ground motion is then scaled to increasing intensity levels and the nonlinear dynamic analysis is repeated until collapse.

\subsection{Performance-Based Seismic Design}

Although damage is expected when designing a structure by current seismic codes, the extent of the damage is not a direct consideration in design. For this reason, design codes and retrofit manual are moving towards to a performance-oriented seismic design, where reinforced concrete bridges are designed to achieve different levels of performance when subjected to different levels of seismic demand (Figure 5.1). A performance level is a specific damage state that is presented qualitatively in terms of physical damage (Lehman et al., 2004). However, the quantitative definition of these qualitative damage states and hence the performance limit needs to be defined with respect to an engineering deformation criteria, i.e. peak strain, displacement, and curvature. Material strain values such as concrete compressive strain and steel tensile 
strain are inherent material properties that can be related to the limit states and have been commonly used in the literature (Kowalsky, 2000). This link between damage state and strain limit allows engineer to know what performance is intended (i.e., what damage is prevented).

Past research has been dedicated for the development of strain-based limit states for reinforced concrete bridge columns, as shown in Table 3.21. As can be seen, in the two-level performance criteria required by the Oregon Department of Transportation for SDC D (ODOT, 2017), a structural performance is described with two discrete performance levels and two seismic hazards, namely Operational Performance Level for a full rupture Cascadia Subduction Zone Earthquake and Life Safety Performance Level for a 1000-year return period event. From the table, it can be seen that ODOT's performance strain limits show a difference in concrete strain between the Operational and the Life Safety performance level of only 0.003 , which was caused by the low concrete confinement, typical of RC columns built prior to 1970. Goodnight and Kowalsky (2015) experimentally evaluated the performance strain limit recommended by Kowalsky (2000) and those results are also presented in Table 3.21. Serviceability strain limits were maintained, while new strain limit expression for Life Safety were developed for the compressive strain at initial yielding of confinement steel and the tension strain preceding bar buckling. Contrary to ODOT criteria, the difference in compressive strain between the Operational and Life Safety performance is greater. This great margin is attributed the database used by Goodnight, which consisted of well-detailed concrete 
columns. On the other hand, good agreement was found between the measured peak tensile strain preceding bar buckling and the new expression developed by the authors.

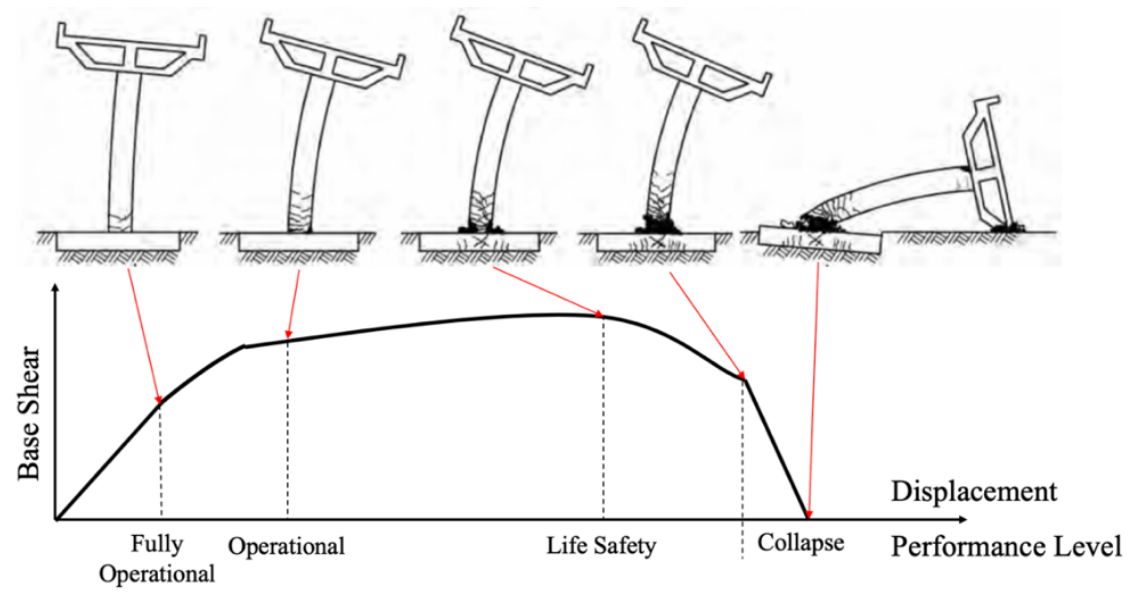

Figure 5.1 Visualization of Performance Based Seismic Design (Adapted from (NCHRP, 2013))

In Section 3.7, each performance limit state was evaluated based on the experimental results reported in this study. Based on the experimental results, strain limits were derived for each performance level for substandard RC columns (Table 3.24). As it was observed in the experimental program, subduction zone earthquake demands induce more number of inelastic cycles compared to crustal demands, and that affected the occurrence of bar buckling, which is not considered by current ODOT's performance limit states. In general, current material strain in most codes do not consider the cumulative damage effects from subduction zone seismic events. An option to address this is to put strain limit data into a probabilistic PBSD format where the damage states and performance levels are linked through fragility or probability of occurrence curves.

\subsection{Seismic Fragility Analysis}

Seismic fragility analysis as emerged as an important seismic risk assessment methodology for highway transportation systems. With the advancement of the 
performance-based earthquake engineering, design criteria are moving towards fragility curves as a means of describing the performance at different levels of seismic input intensity (Mackie \& Stojadinovic, 2005). Fragility curves are defined as statistical probability distributions that indicate the probability of a component, element, or a system to reach or exceed a specific damage state (DS) as a function of engineering demand parameters (EDP), ((Porter et al., 2007); (FEMA P-58 , 2012)). The fragility of a structure or component can be expressed as a conditional probability that a defined limit state (LS) is exceeded for a given level of ground motion intensity (IM), as follows:

$$
\text { Fragility }=P[L S \mid I M]
$$

Different methods and approaches have been developed by different researchers for developing fragility curves such as judgmental, field observations, advance analysis using analytical models as well as hybrid methods. In this study, analytical fragility curves are developed through nonlinear time history analysis (NLTHA). In spite of being one of the most computationally expensive methods, NLTHA is the most reliable method for generating fragility curves (Shinozuka et al., 2000). This method allows the consideration of geometric nonlinearity and material inelasticity to predict the large displacement behavior and the collapse load of bridges accurately under dynamic loading. To reduce the requirement of a large number of ground motions for fragility assessment using NLTHA, the IDA method was used instead. This method is a special type of NLTHA where ground motions are incrementally scaled and a series of analyses is performed at different intensity levels (Vamvatsikos \& Cornell, 2012). Intensity levels are selected to cover the entire range of structural response, from elastic behavior through 
yielding to the failure limit state. Although the actual application of the fragility analyses may vary, all applications follow the basic approach depicted in Figure 5.2.

\subsection{Fragility Analysis for Full-Scale RC Bridge Column}

The main objective of this study is to obtain fragility curves for the full-scale RC bridge column presented in Section 4.3, which may represent a column part of a single or multi-column bridge bent built prior to 1970 in the Pacific Northwest of the United States. Bridges of that era were designed with poor seismic detailing and provided of lap splices whiting plastic hinge zones. The probabilistic fragility analysis of the full-scale substandard RC bridge column was carried out through IDA in OpenSees (2013). The good match between experimental and numerical results of the tested specimens gave confidence for using the calibration parameters in the numerical model of the full-scale column presented in Section 4.3.

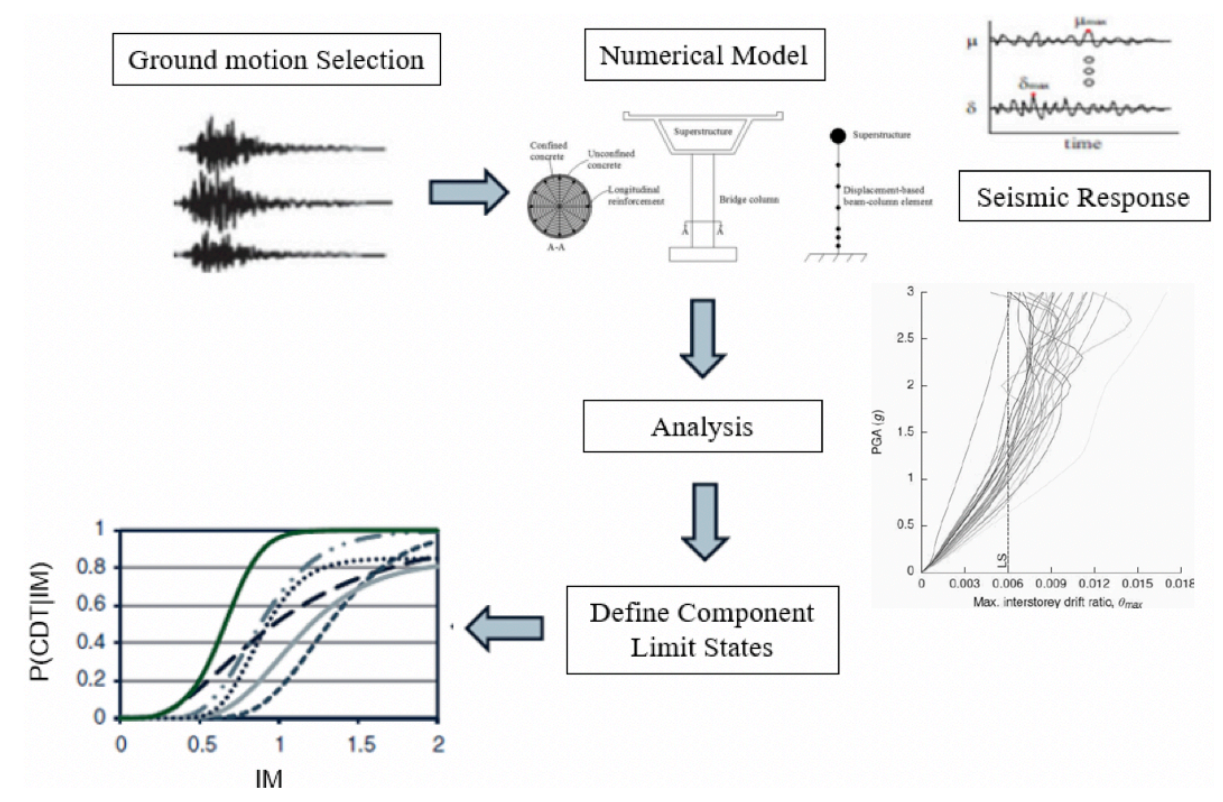

Figure 5.2 Schematic representation of the NLTHA procedure used for Fragility Analysis 


\subsubsection{Selected Earthquake Ground Motions}

Subduction and crustal ground motion records were considered as input for the NLTHA, to contrast the difference in performance under these two demand sources. The subduction set consisted of 20 ground motions, of which 10 are from the Maule, Chile earthquake M8.8 (U. Chile, 2010), and 10 from the Tohoku earthquake M9.0 (K-Net, 2011), in an effort to represent a potential Cascadia earthquake scenario. The crustal set consisted of 20 ground motions that were chosen from the FEMA P695 far-field record (FEMA P695, 2009), (COSMOS, 2012)). The seismic actions were represented by means of 5\% damped elastic response spectra as shown in Figure 5.3. Table 5.1 summarizes the selected ground motion, which also includes the peak ground acceleration (PGA) and duration of each record.

The duration of strong ground motion is widely recognized as an important characteristic affecting the response structures, however there is no wide consensus about what is the proper duration definition of a ground motion. Among the numerous definitions of ground motion duration available in the literature (Bommer \& MartinezPereira, 1999), (Hancock \& Bommer, 2006) (Foschaar et al., 2012), the 5-95\% significant duration (Trifunac \& Brady, 1975) metric was used in this part of the study. This metric is calculated as the interval between the times at which $5 \%$ and $95 \%$ of the Arias Intensity of the ground motion have been recorded, representing $90 \%$ of the total accumulated energy. As expected, subduction ground motions have longer duration than crustal ground motion. The significance of this excitation duration is particularly important for nonlinear structures as the number of response cycles is directly related to 
duration. Further, structural systems or components become increasingly vulnerable given the increasing number of cyclic reversals caused by long duration motions (Bertero et al., 1977), (Marsh \& Gianotti, 1995). Also, longer duration may lead to more damage and risk of collapse (Raghunandan \& Leil, 2013), (Chandramohan et al., 2013).
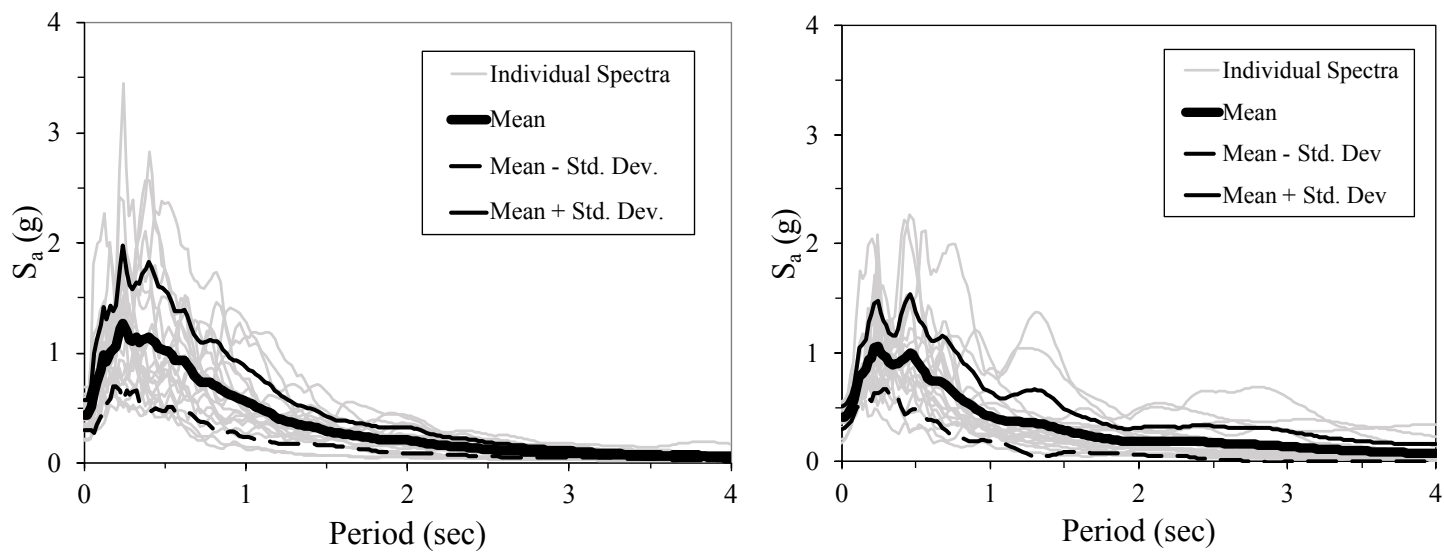

Figure 5.3 Elastic response spectra of the ground motions used in this study (5\% damping ratio).

a) Subduction set, b) Crustal set

\subsubsection{Damage States}

Realization of performance-based seismic design requires methods to quantify the degree of damage. Damage states (DS) for a bridge component should provide a useful qualitative and quantitative representation for that component. These DS are often based on visual representations but they need to be quantified. This can be accomplished through engineering demand parameters that may be expressed by limiting values of quantities such as peak strains. Each damage state may be associated with one or more engineering demand parameter, each of which must be evaluated in order to assess the performance level. In this work, the engineering demand parameters (EDPs) used for measuring the damage state of the substandard bridge column were the steel and concrete strains. The EDP values are based on available literature (NCHRP, 2013) and the 
experimental results presented in Chapter 3. The values for the EDP are shown in Table 5.2. The steel strain for the damage state of collapse was adapted to the strain at the onset of longitudinal bar buckling according to the experimental results reported in Chapter 3 .

Table 5.1 Summary of the selected ground motions records

\begin{tabular}{|c|c|c|c|c|}
\hline Earthquake & Station & $\begin{array}{l}\text { PGA }_{x} \\
(g)\end{array}$ & $\begin{array}{l}\mathrm{PGA}_{y} \\
(\mathrm{~g}) \\
\end{array}$ & $\begin{array}{c}\text { Significant Duration } \\
(\mathrm{sec}) \mathrm{X}(\mathrm{Y})\end{array}$ \\
\hline $\begin{array}{l}\text { Maule, Chile } \mathrm{M}_{\mathrm{w}}=8.8 \\
\text { (subduction) }\end{array}$ & $\begin{array}{l}\text { Constitucion } \\
\text { Curico } \\
\text { Matanzas } \\
\text { Viña Centro } \\
\text { Viña Salto } \\
\end{array}$ & $\begin{array}{l}0.54 \\
0.41 \\
0.34 \\
0.33 \\
0.34 \\
\end{array}$ & $\begin{array}{l}0.35 \\
0.47 \\
0.29 \\
0.22 \\
0.35 \\
\end{array}$ & $\begin{array}{l}60(65) \\
52(50) \\
68(70) \\
25(29) \\
34(30) \\
\end{array}$ \\
\hline $\begin{array}{l}\text { Tohoku, Japan } \mathrm{M}_{\mathrm{w}}= \\
9.0 \text { (subduction) }\end{array}$ & $\begin{array}{l}\text { Iatate } \\
\text { Sukagawa } \\
\text { Takahagi } \\
\text { Iwaki } \\
\text { Mizusawa }\end{array}$ & $\begin{array}{l}0.51 \\
0.50 \\
0.60 \\
0.30 \\
0.35 \\
\end{array}$ & $\begin{array}{l}0.58 \\
0.69 \\
0.53 \\
0.39 \\
0.21 \\
\end{array}$ & $\begin{array}{l}100(98) \\
52(50) \\
68(70) \\
64(85) \\
93(97) \\
\end{array}$ \\
\hline $\begin{array}{l}\text { Cape Mendocino } \\
\text { (crustal) } \\
\text { Chi-Chi, Taiwan } \\
\text { (crustal) } \\
\text { Fruili, Italy (crustal) } \\
\text { Imperial Valley } \\
\text { (crustal) } \\
\text { Kobe, Japan (crustal) } \\
\text { Loma Prieta (crustal) } \\
\text { Northridge (crustal) } \\
\text { San Fernando (crustal) } \\
\text { Superstition Hills } \\
\text { (crustal) }\end{array}$ & $\begin{array}{l}\text { Rio Dell Overpass } \\
\text { CHY101 } \\
\text { Tolmezzo } \\
\text { El Centro Array } 11 \\
\text { Nishi-Akashi } \\
\text { Capitola } \\
\text { Gilroy Array \#3 } \\
\text { Canyon Country-WLC } \\
\text { LA - Hollywood } \\
\text { Poe Road (temp) }\end{array}$ & $\begin{array}{l}0.36 \\
0.51 \\
0.53 \\
0.56 \\
0.41 \\
0.21 \\
0.45 \\
\end{array}$ & $\begin{array}{l}0.38 \\
0.50 \\
0.44 \\
0.37 \\
0.48 \\
0.17 \\
0.30 \\
\end{array}$ & $\begin{array}{c}15(11) \\
30(26) \\
4(5) \\
9(8) \\
10(11) \\
12(13) \\
6(11) \\
6(6) \\
10(11) \\
14(14) \\
\end{array}$ \\
\hline
\end{tabular}


Table 5.2 Damage states and EDPs for fragility curves

\begin{tabular}{c|c|c|c|c}
\hline Damage Level & II & III & IV & V \\
\hline $\begin{array}{c}\text { Damage } \\
\text { Description }\end{array}$ & Slight & Moderate & Extensive & Collapse \\
\hline $\begin{array}{c}\text { Physical } \\
\text { Description }\end{array}$ & $\begin{array}{c}\text { First yield of } \\
\text { tensile } \\
\text { reinforcement }\end{array}$ & $\begin{array}{c}\text { Onset of } \\
\text { Spalling }\end{array}$ & $\begin{array}{c}\text { Complete } \\
\text { (extended) spalling }\end{array}$ & $\begin{array}{c}\text { Bar buckling } \\
\text { Bar fracture } \\
\text { Concrete crushing }\end{array}$ \\
\hline $\begin{array}{c}\text { Steel Strain Limit } \\
\text { (in./in.) }\end{array}$ & 0.0018 & 0.016 & 0.026 & $0.032\left(0.06^{2}\right)$ \\
\hline $\begin{array}{c}\text { Concrete Strain } \\
\text { Limit (in./in.) }\end{array}$ & 0.001 & 0.006 & 0.01 & 0.012 \\
\hline
\end{tabular}

\subsubsection{Fragility Curves}

Incremental dynamic analysis was employed to perform the fragility based seismic performance of the substandard RC bridge column, following the procedure depicted in Figure 5.2. The column model was subjected to two orthogonal horizontal components of the two ground motions sources listed in Table 5.1. As it was mentioned before, the nonlinear time history analyses were performed in OpenSees at increasing levels of ground motion intensity. Based on the peak tensile strain of the longitudinal reinforcement and peak concrete compressive strain demand in column, each damage limit state of the column is assessed. To correlate seismic damage and hazard levels of ground motion, peak ground acceleration (PGA) was chose as the intensity measure (IM) as suggested by Padgett et al. (2008). Then, using the IDA method the fragility curves can be obtained using the damage probability at a given IM level. This damage probability is calculated as the ratio of the number of damage cases "ni" for the damage

\footnotetext{
${ }^{2}$ Strain limit for bar fracture in \#11 - \#18 rebars (AASHTO, 2009), (Priestley et al., 2007)
} 
state " $\mathrm{i}$ " over the number of total simulation cases $\mathrm{N}$ as shown in Eq. (5.2) (Zhang \& Huo, 2009).

$$
P\left[D I \geq L S_{i} \mid I M\right]=\frac{n_{i}}{N} \quad(i=1-4)
$$

In this study, the lognormal cumulative probability distribution function was fitted to the data in order to generate the IDA fragility curves using the maximum likelihood method as suggested by Baker (2015). The exceedance probability values provide the median and dispersion values of the cumulative lognormal probability distribution function used in Eq. (5.3).

$$
P\left[D I \geq L S_{i} \mid I M\right]=\int_{0}^{I M} \frac{1}{i m \sqrt{2 \pi} \xi_{I M}} \exp \left\{-\frac{\left(\ln (\mathrm{im})-\lambda_{I M}\right)^{2}}{2 \xi_{I M}^{2}}\right\} d(i m)
$$

Where, $\lambda_{I M}$ and $\xi_{I M}$ are the standard deviation and mean value of IM to reach the specified damage state based on the log-normal distribution.

Figure 5.4 and Figure 5.5 show the peak tensile strain and peak compressive concrete strain fragility curves for the full-scale substandard RC bridge column, subjected to subduction and crustal records using PGA as intensity measures. Additionally, the median values and dispersion for the moderate and collapse damage states are shown in Table 5.3 and Table 5.4. In order to compare to the dual-level performance criteria used by ODOT (2017), only the Moderate (associated to onset of concrete spalling) and Collapse (associated to bar buckling and concrete crushing) damage states fragility curves are presented. As expected, the substandard column is more vulnerable to subduction ground motions. When considering the peak tensile strain as the EDP, the 
probability of exceeding moderate damage for $\mathrm{PGA}=0.4 \mathrm{~g}$ is about $80 \%$ for subduction records and $60 \%$ for crustal ground motions (Figure 5.4(a)). The same trend is observed in the collapse limit state where the probability of damage is about $15 \%$ for crustal sources and $50 \%$ for subduction sources. This can be explained by the increased number of cycles imposed by subduction ground motions which trigger the initiation of longitudinal bar buckling. Further, this high probability of damage agrees with the results of the experimental program. Same trend is observed when considering the peak compressive concrete strain as the EDP. The probability of exceeding moderate damage for $\mathrm{PGA}=0.4 \mathrm{~g}$ is about $20 \%$ for crustal records and $55 \%$ for subduction ground motions (Figure 5.5(a)). Conversely, for the collapse limit state probability of damage is almost similar (Figure 5.5(b)). This outcome is consistent with the concrete strain limit state proposed by ODOT for Life Safety, since concrete strains at this performance level at low due to low concrete confinement, which is typical of $\mathrm{RC}$ bridge columns built prior to 1970 . As can be seen, for a PGA of $0.4 \mathrm{~g}$, the probability of damage is about $5 \%$ for crustal sources and $15 \%$ for subduction sources. Additionally, the fragility curves also show that the RC bridge column presents a low probability to reach collapse damage limit state for PGAs less than $0.3 \mathrm{~g}$. Thus, results show that RC bridge substructures are more vulnerable to subduction zone earthquakes than for crustal earthquakes.

Furthermore, the results show that considering a lower peak tensile strain for the collapse damage states is suitable when assessing the performance of substandard RC substructures. 


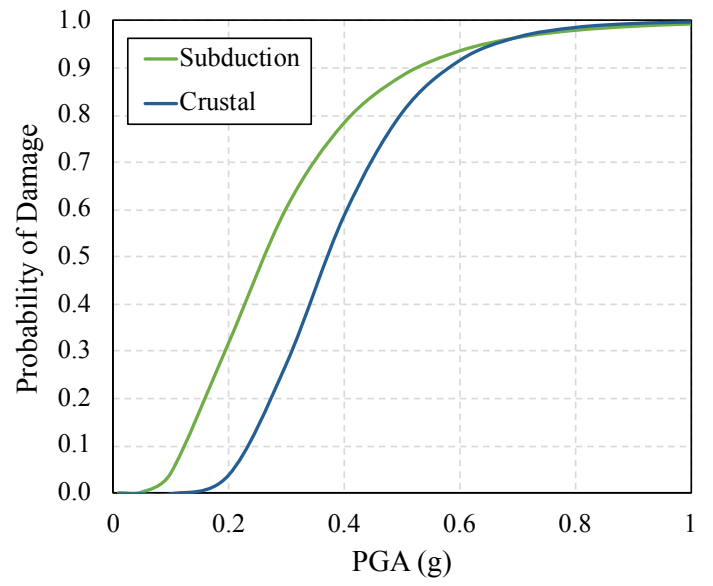

a)

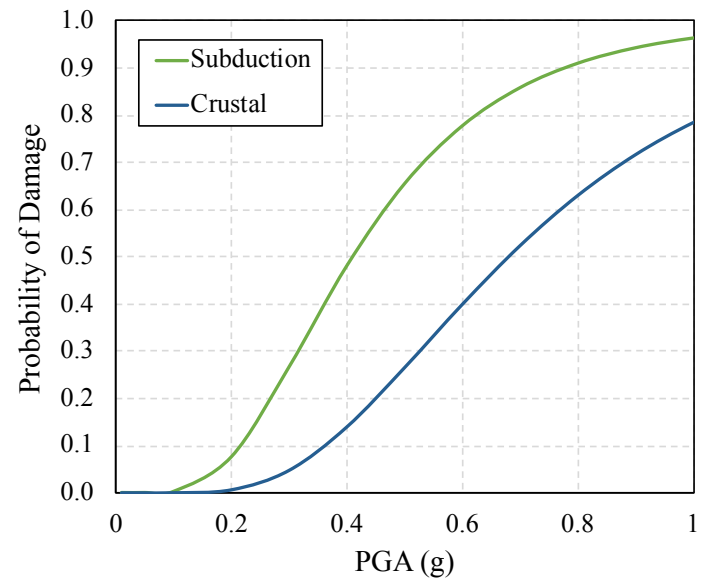

b)

Figure 5.4 Peak tensile steel strain fragility curves. a) Moderate, b) Collapse

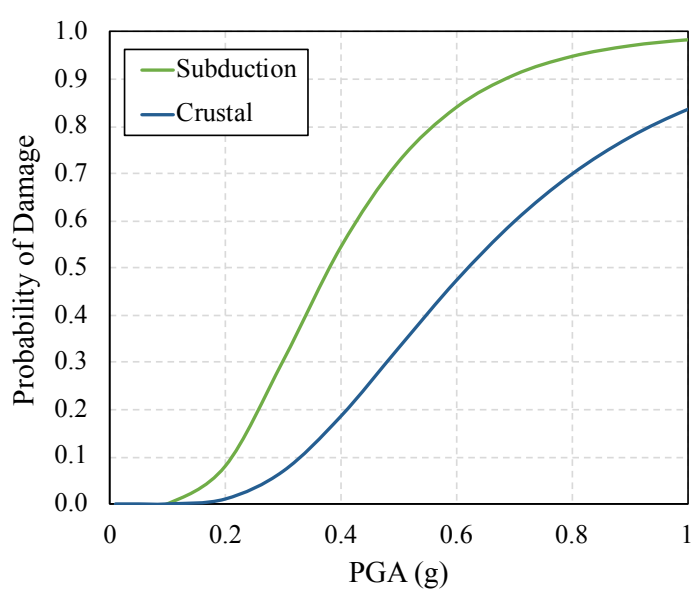

a)

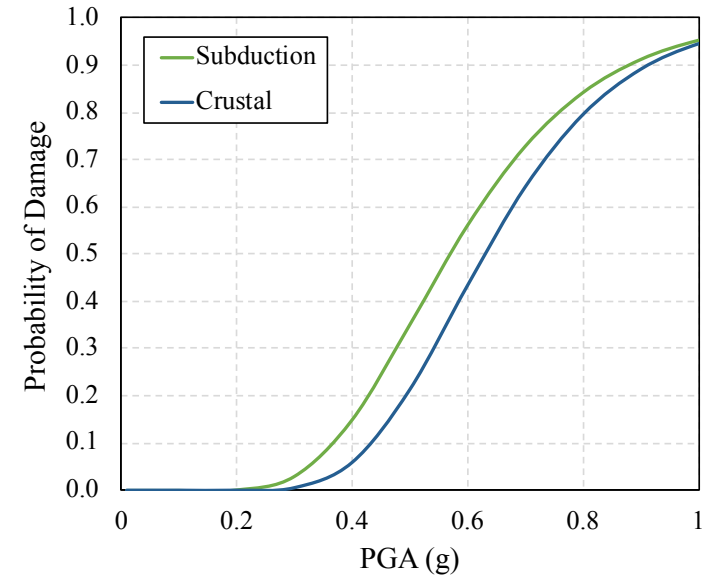

b)

Figure 5.5 Peak compressive concrete strain fragility curves. a) Moderate, b) Collapse

Table 5.3 Fragility curve values for full-scale RC bridge column under subduction records

\begin{tabular}{c|c|c|c|c}
\hline \multirow{2}{*}{ Parameter } & \multicolumn{2}{|c|}{ Steel Strain Damage States } & \multicolumn{2}{c}{ Concrete Strain Damage States } \\
\cline { 2 - 5 } & Moderate & Collapse & Moderate & Collapse \\
\hline Median & 0.26 & 0.59 & 0.38 & 0.57 \\
\hline Dispersion & 0.55 & 0.25 & 0.46 & 0.34 \\
\hline
\end{tabular}


Table 5.4 Fragility curve values for full-scale RC bridge column under crustal records

\begin{tabular}{c|c|c|c|c}
\hline \multirow{2}{*}{ Parameter } & \multicolumn{2}{|c|}{ Steel Strain Damage States } & \multicolumn{2}{c}{ Concrete Strain Damage States } \\
\cline { 2 - 5 } & Moderate & Collapse & Moderate & Collapse \\
\hline Median & 0.37 & 0.83 & 0.62 & 0.63 \\
\hline Dispersion & 0.35 & 0.35 & 0.49 & 0.29 \\
\hline
\end{tabular}

\subsection{Strain Limit Sates Recommendations}

From the results of the fragility based seismic performance of the full-scale substandard RC bridge column, it is seen that RC bridges are more vulnerable to subduction zone earthquakes than for crustal earthquakes. The same analytical framework using the same or an extended set of ground motions can be used with different column and bridge configurations in order to come up with a full performancebased design procedure that takes into consideration the effects of subduction zone earthquakes in the seismic performance of bridges. However, based on the presented experimental and numerical work on a single column configuration, it is recommended to consider the effects of subduction zone earthquakes in the performance assessment of substandard bridges, or when choosing ground motions for nonlinear time-history analysis, especially in regions prone to this type of seismic actions. Thus, for substandard bridges not yet retrofitted or upgraded seismically, the following performance limit recommendation is proposed: for the damage state of collapse, which is related to the ODOT's Life Safety performance level, the maximum strain in the longitudinal reinforcement should be reduced from 0.09 (in./in.) to a value of 0.032 (in./in.) for locations where subduction zone earthquakes are expected, to take into consideration the 
occurrence of bar buckling. Material strain limits for other performance levels are consistent with the results of this study and should be kept without modification.

The recommendation previously proposed is for existing bridges not yet retrofitted or seismically upgraded. Engineers should follow the procedure here indicated in order to consider the strain limit recommendation. For an existing structure evaluation, bridges shall be modelled as close to the actual as-built structural condition as possible. First, an elastic analysis is performed to analyze the strength limit states as well as the service and fatigue limit states. Then, in order to capture the effects of subduction earthquakes, nonlinear static analysis (Pushover Analysis) is required as minimum, although nonlinear time history analysis is preferred. In this nonlinear model, steel and concrete strain shall be determined by the engineer to evaluate the Performance Criteria. In particular, the steel strain demand obtained from the analysis can be compared to the steel limit strain proposed in this study for the Life Safety Performance Level. If the limit is exceeded, provisions with the structure shall be taken given the impending Cascadia Subduction Zone earthquake in the Pacific Northwest of the United States. 


\section{CHAPTER 6. Conclusions and Recommendation for Further Research}

\subsection{Conclusions}

A new inertial mass system (IMS) for dynamic testing using shaking tables was developed and presented. In order to assess the effects of the system on the force and stiffness of specimens, the dynamic equation of motion of the shaking table and the mass system was developed. The inertial loading system was used in shaking table testing of six substandard RC bridge column models, and the results have been shown to provide evidence on the effectiveness of the proposed system. Further, the new system was compared numerically to two popular mass system used in the literature. Numerical simulations have shown that the proposed IMS can reproduce the seismic performance of cantilever columns as if the mass was placed directly on top of the specimen, which represent the ideal scenario for testing these type of bridge components. Therefore, based on the observed behavior and the advantages identified (safety for attaining collapse performance level, small out-of-plane displacements and reduced time for test preparation), the external device can be used suitably as an inertial loading system for dynamic testing using shaking tables.

Six lightly reinforced bridge columns typical to Oregon were subjected to shaking table loading to investigate the effect of subduction ground motion. Two sets of three columns were subjected to one crustal and two subduction zone earthquakes. The first set corresponded to circular columns with continuous reinforcement, all of them with same material properties and dimensions. The seismic performance of these specimens showed 
moderate ductility and was controlled by flexural behavior. For all these specimens, the hysteresis curves were stable and showed acceptable energy dissipation. The second set of specimens included lap splice in the plastic hinge region; two specimens with short lap splice $\left(25 \mathrm{~d}_{\mathrm{b}}\right)$ and one column with relatively long but still deficient lap splice $\left(40 \mathrm{~d}_{\mathrm{b}}\right)$. Specimens with short lap splice showed anticipated lap-splice failure characterized by sudden degradation of their lateral load-carrying capacity after reaching their flexural strength and non-ductile response.

The experimental results also showed that subduction ground motion reduce the displacement capacity of the column as compared to a crustal record. This result is demonstrated by buckling of the longitudinal bars observed in specimens $\mathrm{C} 2 \mathrm{C}-\mathrm{M}$ and C3C-T under subduction demands, which define the failure stage of these specimens. Conversely, failure was not achieved in column C1C-L after the second motion amplitude. For short lap-spliced specimens, the primary mode of failure was consistently a lap-splice failure, however this failure occurred at different motion amplitudes. Specimen C5S1-M failed after motion amplitude A, whereas C4S1-L failed during motion amplitude B. This result also indicates that more damage is expected under subduction motion demands compared to crustal ones. As for the lap splice length, a deficient yet a relatively long lap splice length can improve the performance of the column to the point of moderately ductile behavior despite the duration of the ground motion.

The measured dynamic properties of the specimens (periods and damping) changed with the damage progression. The periods gradually lengthened with increasing 
levels of excitation as a consequence of the stiffness degradation. Moreover, the variation in damping also increased within successive motion amplitudes. The period lengthening was more pronounced for tests using the subduction earthquakes records and can have an impact on the post-earthquake response of the damaged structures including strong aftershocks. As for the dissipated energy, it was found that the total cumulative energy dissipated was significantly affected by the duration of the ground motion due to increased number of loading cycles.

To satisfy the aims of performance-based seismic design, damage levels that interrupt the serviceability of the structure or require repair techniques could be related to engineering criteria. The results of experimental program presented in this work were analyzed to assess the performance levels of substandard bridge columns. Performance strain limits recommendations from ODOT's requirements for new bridges were evaluated using measured reinforcement strains. Based on ODOT's performance criteria, buckling or concrete core crushing represents the point at which repair is necessary, interrupting the use of the structure, but not necessarily posing a safety concern. Dynamic test results exhibited bar buckling after reversal from peak tensile strain for subduction ground motions, while complete spalling of cover concrete was observed for the crustal motion. For the Operational performance level, computed concrete compressive strains were higher than the values proposed by ODOT and Goodnight, being higher for the specimens under subduction motion demands. As for steel tensile strains, the computed strains were higher than the values cited by Goodnight only for the specimens subjected to subduction ground motions. For the Life Safety performance level, recorded tensile 
strain preceding bar buckling exceed the expression proposed by Goodnight for subduction records and were found to be 0.035 and 0.029 , for $\mathrm{C} 2 \mathrm{C}-\mathrm{M}$ and $\mathrm{C} 3 \mathrm{C}-\mathrm{T}$ respectively. This finding ratifies the hypothesis that more structural damage is expected from subduction ground motions. Furthermore, results indicate that increased number of loading cycles imposed by subduction demands has a strong influence on bar buckling. For specimens with short lap-splice length at hinge, core crushing limit state was defined due to lap-splice failure with extensive core damage. Even though computed concrete compressive strains for these specimens were similar, the limit state occurred at different motion amplitudes. Despite that the concrete compressive strain for C5S1-M does not exceed for the Life Safety performance level, its loss of load-carrying capacity revealed the vulnerability of seismically deficient columns subjected to subduction demands.

Finally, from the test results can be concluded that ground motion duration affects the performance of reinforced concrete bridge columns. Base on the experimental evidence presented in this study, more structural damage is expected under subduction seismic events. This outcome supported by the values of CDD index for each specimen, which were considerably higher for subduction demands. Furthermore, the material strain support this finding, since current strain limit states for the two-level performance criteria evaluated herein were exceeded when considering subduction ground motions.

The numerical model calibrated against experimental test results was able to reasonably reproduce the initial stiffness, and strength as well as the stiffness degradation of the specimens. The distributed plasticity model also includes the effect of low-cycle fatigue on the reinforcing bars and showed very good agreement with the measured steel 
strains. Additionally, degradation parameters were needed to appropriately capture the damage on substandard columns. Since pre-1970 columns were built with poor seismic detailing and lap splices within plastic hinge zones, it is suggested that values of 0.008 and 0.005 for the damage 1 and damage2, respectively, to represent the behavior of columns. These higher damage parameters should be incorporated in areas where subduction earthquakes are expected.

Fragility curves that were developed using nonlinear analysis showed that the full-scale substandard bridge column considered in this study is more susceptible to subduction zone ground motions than crustal ground motions. As a result, the analyses of the fragility curves revealed the necessity of revising the steel strain limits for the collapse damage state, which is related to longitudinal bar buckling. The fragility curves confirmed the finding of the experimental program, revealing that subduction zone earthquakes impose more inelastic cycles that trigger the initiation of longitudinal bar buckling. Moreover, based on the experimental and numerical results, subduction zone ground motion effects are considered to have a significant effect on the performance of bridge columns. Therefore, it is recommended to consider the effects of subduction zone earthquakes in the performance assessment of substandard bridges, or when choosing ground motions for nonlinear time-history analysis, especially in regions susceptible to be struck by subduction zone mega earthquakes. Finally, for substandard bridges not yet retrofitted or upgraded seismically, the following performance limit recommendation is proposed: for the damage state of collapse, which is related to the ODOT's Life Safety performance level, the maximum strain in the longitudinal reinforcement should be 
reduced from 0.09 (in./in.) to a value of 0.032 (in./in.) for locations where subduction zone earthquakes are expected, to take into consideration the occurrence of bar buckling. Material strain limits for other performance levels are consistent with the results of this study and should be kept without modification.

\subsubsection{Recommendations for Further Research}

Future research on the effects of subduction zone earthquakes in the seismic performance of bridges are summarized below:

Since there is a vast inventory of bridges that have been built with no enough attention for seismic demands in the State of Oregon, it is recommended to perform a prioritization of the bridges in inventory, based upon the proposed steel strain limit for ODOT's Life Safety performance level. Thus, a ranking of the bridges for retrofitting and seismic upgrading can be obtained for the decision makers, and so the limited costs for infrastructure upgrading are managed.

Due to laboratory constraints, the experimental program only considered horizontal components of the ground motions for the shake table tests. It is recommended to include the vertical component in future experimental campaigns, since the vertical components of recent mega subduction earthquakes have caused more damage than the other components.

Even though there has been shake table experiments on bridge bents and full bridges, it is recommended to conduct experiments on full bridges including subduction ground motion as input for the shake table. 
In this study, rigid foundation was considered for both experimental and numerical studies. It is recommended to study the effects of subduction ground motions in bridges with flexible foundations or including soil-structure interaction, such as soil liquefaction.

More investigation on the effect of subduction ground motions on structural response is required based on specific characterization of the seismic hazard in regions expected to be struck by the type of seismic events. Also, include different column design parameters, such as aspect ratio, level of axial load, hoop size and spacing, that would affect the comparison between crustal and subduction demands.

$>$ Fragility curves are a reliable tool for risk assessment. It is recommended to perform probability fragility seismic assessment on full bridges to contrast the difference in damage induced by subduction and crustal earthquakes, and further validate the results presented in this study. 


\section{REFERENCES}

AASHTO, 2009. Guide Specifications for LRFD Seismic Bridge Design, s.1.: American Association of State Highway and Transportation Officials.

ACI, 2014. Building Code Requirements for Structural Concrete and Commentary.

Detroit, MI: ACI 318-14.

American Association of State and Highway Transportation Officials, A., 1996. Standard Specifications for Highway Bridges. 16th edition ed. Washington, DC.: American Association of State and Highway Transportation Officials.

Andrawes, B., Shin, M. \& Wierschem, N., 2010. Active Confinement of Reinforced Concrete Bridge Columns Using Shape Memory Alloys. Journal of Bridge Engineering, 15(1), pp. 81-89.

ASTM A1064, 2017. Standard Specification for Carbon-Steel Wire and Welded Wire Reinforcement, Plain and Deformed, for Concrete. West Conshohocken, PA: s.n. ASTM A615, 2018. Standard Specification for Deformed and Plain Carbon-Steel Bars for Concrete Reinforcement. West Conshohocken, PA: s.n.

Atwater, B. et al., 2005. The orphan tsunami of 1700: Japanese clues to a parent earthquake in North America. U.S. Geological Survey Professional Paper 1707, p. 135 . 
Bairrao, R. \& Vaz, C., 2000. Shaking table testing of civil engineering structures-the LNEC 3D simulator experience. Auckland, New Zeland, Proceedings of the 12th World Conference on Earthquake Engineering.

Baker, J., 2015. Efficient analytical fragility function fitting using dynamic structural analysis.. Earthquake Spectra, 31(1), pp. 579-599.

Bazaez, R., 2017. Achieving Operational Seismic Performance of RC Bridge Bents Retro\#\$ed with Buckling-Restrained Braces. s.l.:PhD Dissertation, Department of Civil and Environmental Engineering, Portland State University, Portland, OR.

Bazaez, R. \& Dusicka, P., 2016. Cyclic Loading for RC Bridge Columns Considering Subduction Megathrust Earthquakes. Journal of Bridge Engineering, 21(5), p. 04016009 .

Berry, M. \& Eberhard, M., 2008. Performance Modeling Strategies for Modern Reinforced Concrete Bridge Columns, University of California, Berkeley: Pacific Earthquake Engineering Research Center, PEER Report 2007/07.

Bertero, V., Popov, E., Wang, T. \& Vallenas, J., 1977. Seismic Design Implications of Hysteretic Behavior of Reinforced Concrete Structural Walls. India, s.n., pp. Vol II, 1898-1904.

Bommer, J. \& Martinez-Pereira, A., 1999. The effective duration of earthquake strong motion. Journal of Earthquake Engineering, 3(2), pp. 127-72.

Boroscheck, R., Contreras, V., Kwak, D. \& Stewart, J., 2012. Strong Ground Motion Attributes of the 2010 Mw 8.8 Maule, Chile, Earthquake. Earthquake Spectra, Volume 28, pp. 519-538. 
Bubkle, I. y otros, 2012. Structural Performance of Bridges in the Offshore Maule of 27 february. Earthquake Spectra, 28(S1), pp. S333-S552.

Caccese, V. \& Harris, H., 1990. Earthquake simulation testing of small-scale reinforced concrete structures. ACI Structural Journal, 87(1), pp. 72-79.

Caltrans, 1996. Earthquake Retrofit Guidelines for Bridges, SAcramento, California: California Department of Transportation.

Caltrans, 2013. Seismic Design Criteria, Sacramento, California: California Department of Transportation.

Carrillo, J., Gonzalez, G. \& Llano, L., 2012. Evaluation of Mass-Rig Syatems for Shake Table Experiments. Dyna, 79(176), pp. 159-167.

Chai, Y. H., Priestley, M. J. N. \& Seible, F., 1991. Seismic Retrofit of Circular Bridge Columns for Enhanced Flexural Performance. ACI Structural Journal, 88(5), pp. $572-584$.

Chai, Y., Priestley, M. \& Seible, F., 1991. Flexural Retrofit of Circular Reinforced Concrete Bridge Columns by Steel Jackets, University of California, San Diego: Department of Applied Mechanics and Engineering Sciences.

Chandramohan, R., Baker, J. \& Deierlein, G., 2016. Quantifying the influence of ground motion duration on structural collapse capacity using spectrally equivalent records. Earthquake Spectra, 32(2), pp. 927-950.

Chandramohan, R., Lin, T., Baker, J. \& Deierlein, G., 2013. Influence of ground motion spectral shape and duration on seismic collapse risk. Tokyo, Japan, 10th International Conference on Urban Earthquake Engineering. 
Charney, F. A., 2008. Unintended Consequences of Modeling Damping in Structures. ASCE Journal of Structural Engineering, 134(4), pp. 581-592.

Chopra, A., 2001. Dynamic of Structures. Theory and Applications to Earthquake Engineering. Second Edition. s.1.:Prentice-Hall.

Coffin, L. F., 1962. Low cycle fatigue-A review. Applied Material Research, 1(3), pp. $129-141$.

Coffman, H., Marsh, M. \& Brown, C., 1991. Seismic Durability of Retrofitted R.C. Columns, s.1.: Washington State Department of Transportation.

Computers and Structures Inc., C., 2011. SAP2000 Integrated Software for Structural Analysis and Design. Berkeley, CA: s.n.

COSMOS, 2012. Consortium of Organizations for Strong Motion Observation Systems. [Online] Available at: http://www.cosmos-eq.org/

Dobry, R., Idriss, I. \& Ng, E., 1978. Duration characteristics of horizonal components of strong-motion earthquake records. Bulletin of the Seismological Society of America, 68(5), pp. 1487-1520.

EERI, 2010. Learning from Earthquakes: The Mw 8.8 Chile Earthquake of February 27, 2010, Oakland, CA: Earthquake Engineering Research Institute (EERI).

Elwood, K. \& Eberhard, M., 2009. Effective Stiffness of Reinforced Concrete Columns. ACI Structural Journal, 106(4), pp. 476-484.

FEMA P-58 , 2012. Seismic Performance Assessment of Buildings, Washington, D.C.: Federal Emergency Management Agency. 
FEMA P695, 2009. Quantification of Building Seismic Performance Factors,

Washington, D.C.: Federal Emergency Managment Agency.

FHWA, 2006. Seismic Retrofitting Manual for Highway Structures: Part 1-Bridges, Department of Transportation, McLean, Virginia: Federal Highway Administration. Publication No. FHWA-HRT-06-032.

Foschaar, J., Baker, J. \& Deierlein, G., 2012. Preliminary assessment of ground motion duration effects on structural collapse. Lisboa, Portugal, In: 15th World Conference on Earthquake Engineering.

Ghannoum, W. M., 2007. Experimental and Analytical Dynamic Collapse Study of a Reinforced Concrete Frame with Light Transverse Reinforcement. s.1.:PhD Dissertation, Department of Civil and Environmental Engineering, University of California, Berkeley.

Gobierno de Chile, 2010. Plan de Reconstrucion Terremoto y Maremoto del 27 de Febrero de 2010, s.1.: s.n.

Goldfinger, C. et al., 2008. Late Holocene Rupture of the Northern San Andreas Fault and Possible Stress Linkage to the Cascadia Subduction Zone. Bulletin of the Seismological Society of America, 98(2), pp. 861-889.

Goodnight, J. \& Kowalsky, M., 2015. Strain Limit States for Circular RC Bridge Columns. Earthquake Spectra, 32(10.1193/030315EQS036M.).

Goodnight, J., Kowalsky, M. \& Nau, J., 2013. Effect of Load History on Performance Limit States of Circular Bridge Columns. Journal of Bridge Engineering, 18(Special Section), pp. 1383-1396. 
Hachem, M., Mahin, S. \& Moehle, J., 2003. Performance of circular reinforced concrete bridge columns under bidirectional earthquake loading, Berkeley, CA: Pacific Earthquake Engineering Research Center.

Hancock, J. \& Bommer, J., 2006. A state-of-knowledge review of the influence of strongmotion duration on structural damage. Earthquake Spectra, 22(3), pp. 827-45.

Hancock, J. \& Boomer, J., 2007. Usign Spectral Matched Records to Explore the Influence of Strong-Motion Duration on Inelastic Structural Response. Soil Dynamics Earthquake Engineering, 27(4), pp. 291-299.

Haroun, M. A. \& Elsanadedy, H. M., 2005. Fiber-Reinforced Plastic Jackets for Ductility Enhancement of Reinforced Concrete Bridge Columns with Poor Lap-Splice Detailing. Journal of Bridge Engineering, 10(6), pp. 749-757.

Heaton, T. \& Hartzell, S., 1986. Source Characteristics of Hypothetical Subduction Earthquakes in the Northwestern United States. Bulletin of the Seismological Society of America, 76(3), pp. 675-708.

Heaton, T. \& Kanamori, H., 1984. Seismic Potential Associated with Subduction in the Northwestern United States. Bulletin of the Seismological Society of America, 74(3), pp. 933-941.

Hose, Y. \& Seible, F., 1999. Performance Evaluation Database for Concrete Bridge Components and systems under Simulated Seismic Loads, San Diego: Pacific Earthquake Engineering Research Center. 
Hoshikuma, J. \& Guangfeng, Z., 2013. Performance of Seismic Retrofitted Highway Bridges Based on Observation of Damage due to the 2011 Great East Japan Earthquake. Journal of JSCE, Volume 1, pp. 343-352.

Hou, H. \& Qu, B., 2015. Duration effect of spectrally matched ground motions on seismic demands of elastc perfectly plastic SDOFS. Engineering Structures, Volume 90, pp. 48-60.

Iervolino, I., Manfredi, G., Cosenza, E. \& Iervolino, I., 2006. Ground Motion Duration Effects on Nonlinear Seismic Response. Earthquake Engineering and Structural Dynamics, 35(1), pp. 21-38.

Japan Road Association, J., 2002. Design Specifications of Bridges, Part V. Seismic Design. s.1.:s.n.

Japan Society of Civil Engineers (JSCE), 2011. Report on Reconnaissance Damage Investigation to Civil Engineering Facilities Resulted from 2011 Great East Japan Earthquake, Tokyo, Japan: Earthquake Engineering Research Committee.

Kawashima, K. \& Buckle, I., 2013. Structural Performance of Bridges in the Tohoku-Oki Earthquake. Earthquake Spectra, Volume 29, pp. S315-S338.

Kawashima, K. et al., 2011. Damages of Bridges during 2011 Great East Japan Earthquake. Tsukuba Science City, Japan, UJNR.

Kawashima, K. \& Unjoh, S., 1997. The damage of highway bridges in the 1995 Hyogoken-nanbu earthquake and its impact on Japanese seismic design. Journal of Earthquake Engineering, Volume 1, pp. 505-541. 
Kawashima, K. U. S., Hoshikuma, J. \& Kosa, K., 2010. Damage Characteristics of Bridges due to 2010 Chile Earthquake. [Online] Available at: http://www.pwri.go.jp/eng/ujnr/tc/g/pdf/26/26-6-2_hoshikuma.pdf [Accessed 2018].

K-Net, 2011. Kyoshin Network Database, National Research Institute for Earth Science and Disaster Prevention. [Online] Available at: http://www.k-net.bosai.go.jp/ [Accessed 2018].

Kowalsky, M., 2000. Deformation limit states for circular reinforced concrete bridge columns. Journal of Structural Engineering, 126(8), pp. 869-878.

Kunnath, S., El-Bahy, A., Taylor, A. \& Stone, W., 1997. Cumulative Seismic Damage of Reinforced Concrete Damage Piers, Gaithersburg, MD: Building and Fire Research Laboratory, National Institute of Standards and Technology.

Laplace, P., Snaders, D., Saiid, M. \& Douglas, B., 1999. Shake Table Testing of Flexure Dominated Reinforced Concrete Bridge Columns, Reno, NV: Center for Earthquake Engineering Research.

Lehman, D. \& Moehle, J., 2000. Seismic Performance of Well-Confined Concrete Bridge Columns, University of California, Berkeley: Pacific Earthquake Engineering Research Center, Report No. PEER 1998/01.

Lehman, M. et al., 2004. Experimental Evaluation of the Seismic Performance of Reinforced Concrete Bridge Columns. Journal of Structural Engineering, 130(6), pp. 869-879. 
Leonard, L., Currie, C., Mazzotti, S. \& Hyndman, R., 2010. Rupture are amd displacement of past Cascadia great earthquakes from coastal coseismic subsidence. Geological Society of America, Volumen 122, pp. 2079-2096.

Mackie, K. \& Stojadinovic, B., 2005. Fragility basis for california highway overpass bridge seismic decision making, Berkeley, CA: Pacific Earthquake Engineering Research Center. University of California.

MacRae, G., Hodge, C., Priestley, M. J. N. \& Seible, F., 1994. Shake Table Tests of AsBuilt and Retrofitted Configuration, California, San Diego: Structural System Research project. Report No. SSRP/18.

Mander, J., Priestley, M. \& Park, R., 1988. Theoretical Stress-Strain Model for Confined Concrete. Journal of Structural Engineering, 114(3), pp. 1804-1826.

Manson, S. S. \& Hirschberg, M. H., 1963. Fatigue behavior in strain cycling in the lowand intermediate-cycle range.. New York, The 10th Sagamore Army Research Conference: Fatigue-An Interdisciplinary Approach, pp. 13-16.

Marsh, M. L. \& Gianotti, C. M., 1995. Inelastic Structural Response to Cascadia Subduction Zone Earthquakes. Earthquake Spectra, 11(1), pp. 63-89.

Mehary, S., Dusicka, P. \& Bazaez, R., 2018. Effect of Subduction Earthquake-Based Loading History on Substandard RC Square Columns. Journal of Bridge Engineering, pp. 23(3), 04017146.

Miner, M. A., 1945. Cumulative damage in fatigue. Journal of applied mechanics, 12(3), pp. 159-164. 
Ministerio de Obras Públicas, M., 2002. Manual de Carreteras (Manual of Highways), Volumen $N^{\circ} 3$, Instrucciones y Criterios de Diseño. s.1.:Dirección de Vialidad, Ministerio de Obras Públicas, Chile (in Spanish)..

Mohammed, M., 2016. Thesis: Effect of Earthquake Duration on Reinforced Concrete Bridge Columns, Reno, Nevada: University of Nevada, Reno.

Mosalam, K., Naito, C. \& Khaykina, S., 2002. Bidirectional cyclic performance of reinforced concrete bridge column-superstructure subassemblies. Earthquake Spectra, 18(4), pp. 663-687.

NCHRP, N. C. H. R. P., 2013. Performance-Based Seismic Bridge Design, Synthesis 440, Washington, D.C.: Transportation Research Board, National Academy of Sciences.

Neuenhofer, A. \& Filippou, F., 1997. Evaluation of nonlinear frame finite-element.. Journal of Structural Engineering, 123(7), pp. 958-966.

ODOT, 2015. Bridge Design and Drafting Manual. Section 1 - Design, Oregon: Oregon Department of Transportation.

ODOT, 2017. Bridge Design and Drafting Manual, Salem, Oregon: Oregon Department of Transportation.

OpenSees, 2013. Open System for Earthquake Engineering Simulation, Pacific Earthquake Engineering Research Center, University of California, Berkeley V. 2.5.0. [Online] Available at: http://OpenSees.berkeley.edu 
Ou, Y. et al., 2013. Ground Motion Duration Effects on Hysteretic Behavior of Reinforced Concrete Bridge Columns. Journal of Structural Engineering, 140(3), p. 04013065 .

Özer, E., Soyöz, S. \& Çelebi, M., 2012. Effect of Strong Ground Motion Duration on Structural Damage. Lisboa, Portugal, 15th World Conference on Earthquake Engineering.

Padgett, J., Nielson, B. \& DesRoches, R., 2008. Selection of optimal intensity measures in probabilistic seismic demand models of highway bridge portfolios. Earthquake Engineering \& Structural Dynamics, 37(5), pp. 711-725.

Park, R., 1989. Evaluation of ductility of structures and structural assemblages form laboratory testing. Bull. N. Z. Natl. Soc. Earthquake Eng., 22(3), pp. 155-166.

Petrini, L., Maggi, C., Priestley, M. J. N. \& Calvi, G. M., 2008. Experimental Verification of Viscous Damping Modeling for Inelastic Time History Analyzes. Journal of Earthquake Engineering, 12(1), pp. 125-145.

Porter, K., Kennedy, R. \& Bachman, R., 2007. Creating fragility functions for performance-based earthquake engineering. Earthquake Spectra, 22(2), pp. 471489.

Priestley, M. J. N., Calvi, G. M. \& Kowalsky, M. J., 2007. Displacement-based seismic design of structures. s.1.:IUSS Press, Italy..

Priestley, M. J. N., Seible, F. \& Calvi, G. M., 1996. Seismic Design and Retrofit of Bridges. New York: Wiley. 
Priestley, M., Seible, F. \& Uang, C., 1994. The Northridge Earthquake of January 17, 1994: Damage Analysis of Selected Freeway Bridges, University of California, San Diego: Structural Systems Research Project, Report SSRP-94/06.

Raghunandan, M. \& Leil, A., 2013. Effect of Ground Motion Duration on EarthquakeInduced Structural Collapse. Structural Safety, Volume 41, pp. 119-133.

Ranf, R., Eberhard, M. \& Stanton, J., 2006. Effects of Displacement History on Failure of Lightly Confined Bridge Columns. ACI Special Publication, 236(2), pp. 23-42.

Sakai, J., Mahin, S. \& Espinoza, A., 2005. Earthquake Simulator Tests on Reducing Residual Displacements of Reinforced Concrete Bridge Columns, Berkeley, CA: Pacific Earthquake Engineering Research Center.

Sakai, J. \& Unjoh, S., 2006. Earthquake Simulation Test of Circular Reinforced Concrete Bridge Columns under Multidirectional Seismic Excitations. Earthquake Engineering and Engineering Vibrations, 5(1), pp. 103-110.

Schanack, F., Valdebenito, G. \& Alvial, J., 2012. Seismic Damage to Bridges during the 27 February 2010 Magnitude 8.8 Chile Earthquake. Earthquake Spectra, 28(1), pp. 301-315.

Schoettler, M., Restrepo, J., Guerrini, G. \& Duck, D., 2015. A Full-Scale, Single-Column Bridge Bent Tested by Shake-Table Excitation, Berkeley, CA: Pacific Earthquake Engineering Research Center.

Scott, M. \& Fenves, G., 2006. Plastic Hinge Integration Methods for Force-Based BeamColumn Elements. Journal of Structural Engineering, 132(2), pp. 244-252. 
Sezen, H., 2002. Seismic Response and Modeling of Reinforced Concrete Building Columns, University of California, Berkeley: PhD. Dissertation, Department of Civil and Environmental Engineering, .

Shinozuka, M., Feng, M. Q., Kim, H. \& Kim, S., 2000. Nonlinear Static Procedure for Fragility Curve Development. Journal or Engineering Mechanics, December, 126(12), pp. 1287-1295.

Simons, M. et al., 2011. The 2011 Magitude 9.0 Tohoku-Oki Earthquake: Mosaicking the Megathrust from Seconds to Centuries. Science, Volume 332, pp. 1421-1425.

Sozen, M., Monteiro, P., Moehle, J. \& Tang, H., 1992. Effects of Cracking and Age on Stiffness of Reinforced Concrete Walls Resisting In-Plane Shear. Orlando, FL, Proceedings, Fourth Symposium on Current Issues Related to Nuclear Power Plant Structures, Equipment and Piping.

Stapleton, S., McDaniel, C., Cofer, W. \& McLean, D., 2005. Performance of Lightly Confined Reinforced Concrete Columns in Long-Duration Subduction Zone Earthquakes. Journal of the Transportation Research Board, Issue 1928, pp. 185192.

Stephens, J. \& Yao, J., 1987. Damage assessment using response measurements. Journal of Structural Engineering, 113(4), pp. 787-801.

Taucer, F., Spacone, E. \& Filippou, F., 1991. A fiber beam-column element for seismic response analysis of reinforced concrete structures, Berkeley, CA: Earthquake Engineering Research Center, College of Engineering, University of California. Report 91-17. 
Thompson, T. J., 2004. Thesis: The effects of long-duration earthquakes on concrete bridges with poorly confined columns, Washington: Washington State University.

Trifunac, M. \& Brady, A., 1975. A study on the duration of strong earthquake ground motion. Bulletin of the Seismological Society of America, 65(3), pp. 581-626.

U. Chile, 2010. University of Chile, Earthquakes of Chile. [Online] Available at: http://terremotos.ing.uchile.cl/registros/164

U.S. Department of Transportation (FHWA), 2011. Postearthquake Reconnaissance Report on Transportation Infrastructure. [Online] Available at: Infrastructure Impact of the February 27, 2010, Offshore Maule Earthquake in Chile

Unjoh, S., 2012. Repair and Retrofit of Bridges Damaged by the 2010 Chile Maule Earthquake. Tokyo, Japan, Proceedings of the International Symposium on Engineering Lessons Learned from the 2011 Great East Japan Earthquake.

USGS, 1999. United States Geologycal Survey. [Online] Available at: https://pubs.usgs.gov/gip/dynamic/understanding.html [Accessed 2018].

USGS \& Wilshire, H., 1989. USGS page-Oakland. [Online] Available at: http://pubs.usgs.gov/dds/dds-29/screens/022sr.jpeg [Accessed 2018].

Vamvatsikos, D. \& Cornell, A., 2012. Incremental dynamic analysis. Earthquake Engineering \& Structural Dynamics, 31(3), pp. 491-514.

WSDOT, 2006. Bridge Design Manual M 23-50, s.1.: s.n.

Zhang, J. \& Huo, Y., 2009. Evaluating effectiveness and optimum design of isolation devices for highway bridges using the fragility function method. Engineering Structures, 31(8), pp. 1648-1660. 
Zhao, J. \& Sritharan, S., 2007. Modeling of strain penetration effects in fiber-based analysis of reinforced concrete structures. ACI structural journal, 104(2), p. 133. 


\section{APPENDIX. Additional Result from Numerical Model Calibration}

This appendix shows additional results of the numerical calibrations of test specimens presented in Chapter 4.

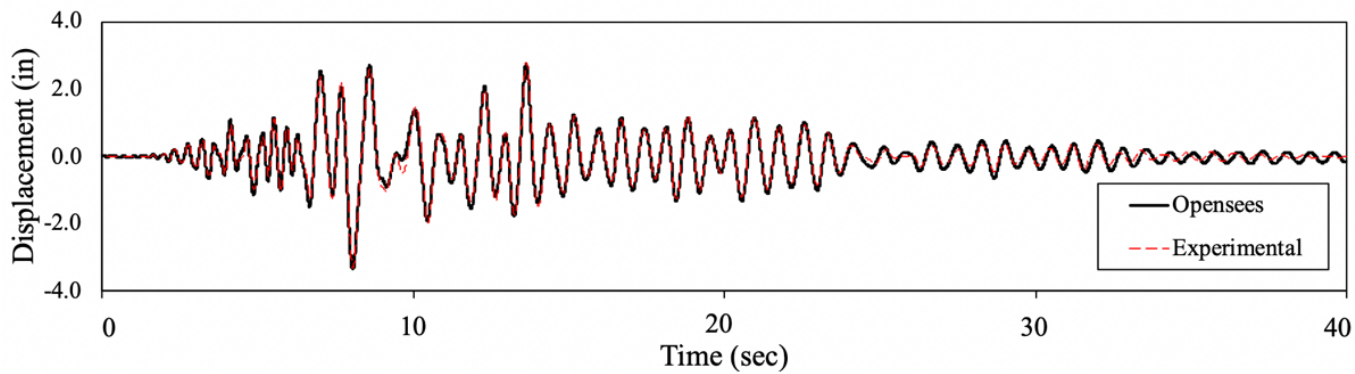

a)

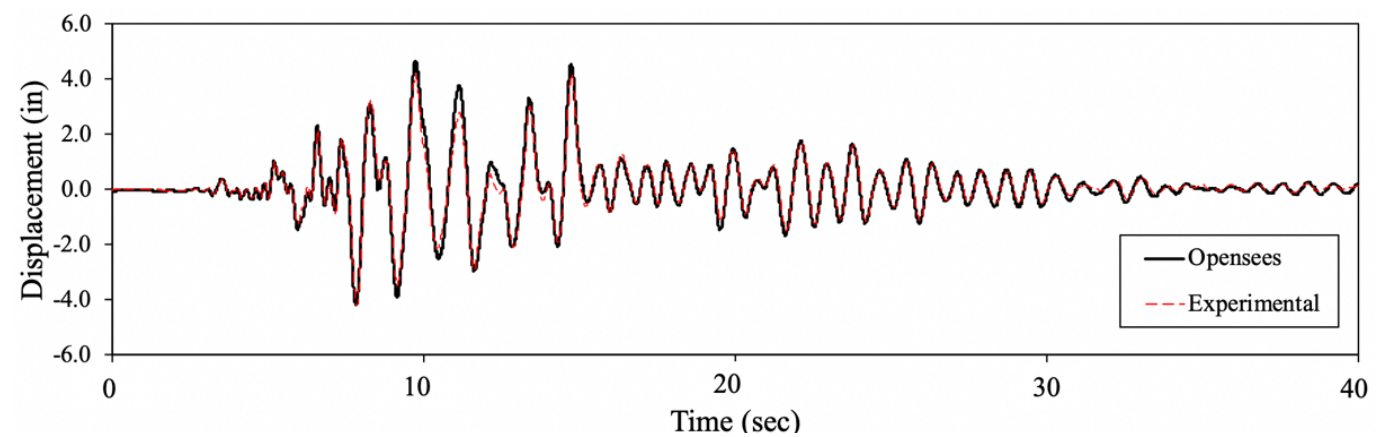

b)

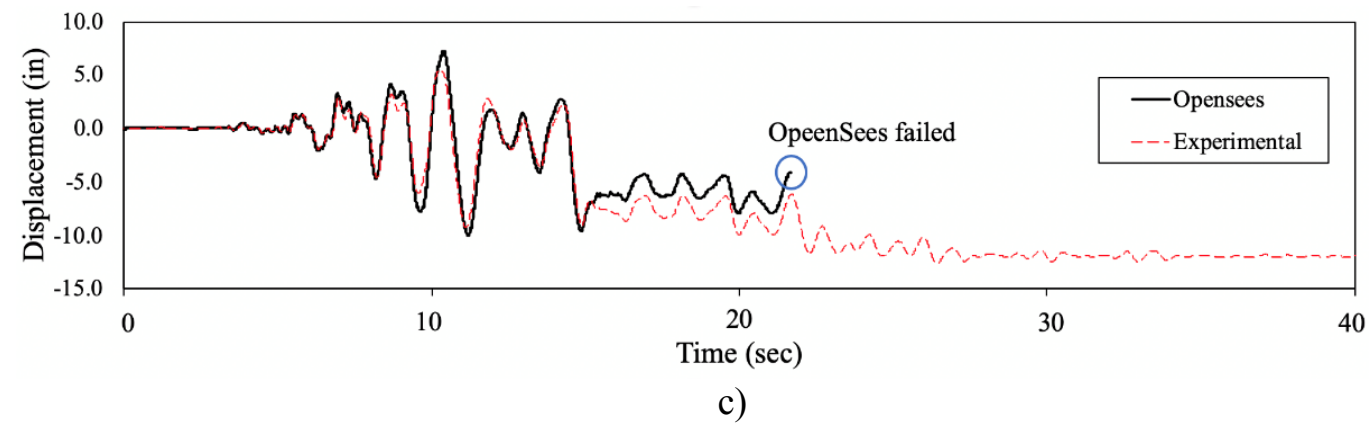

Figure 0.1 Displacement histories comparison for Column C1C-L for all amplitude motions 


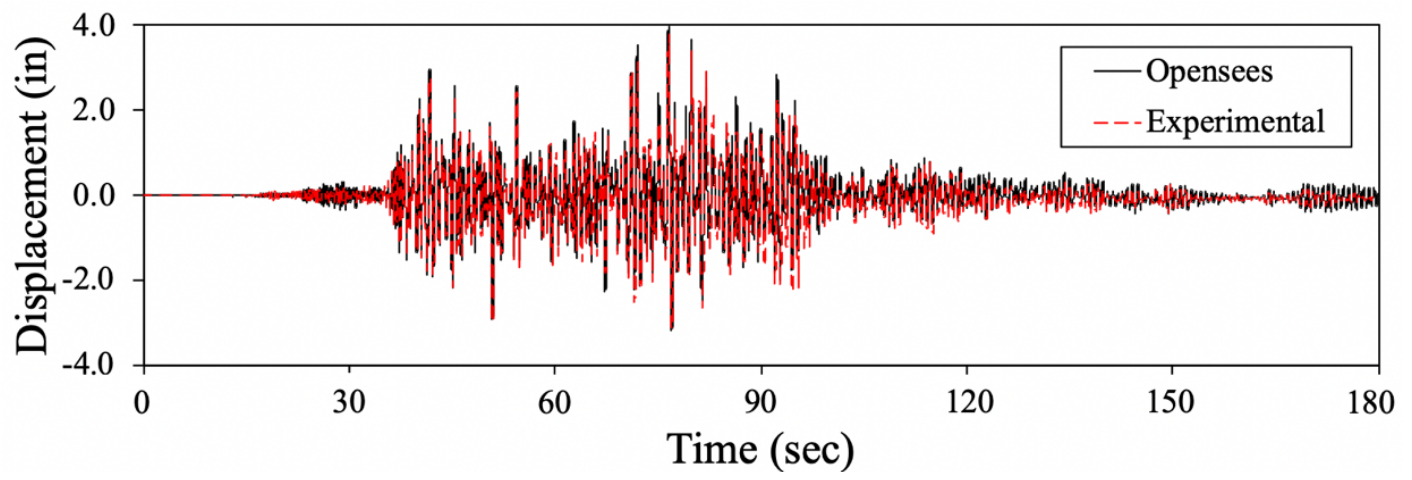

a)

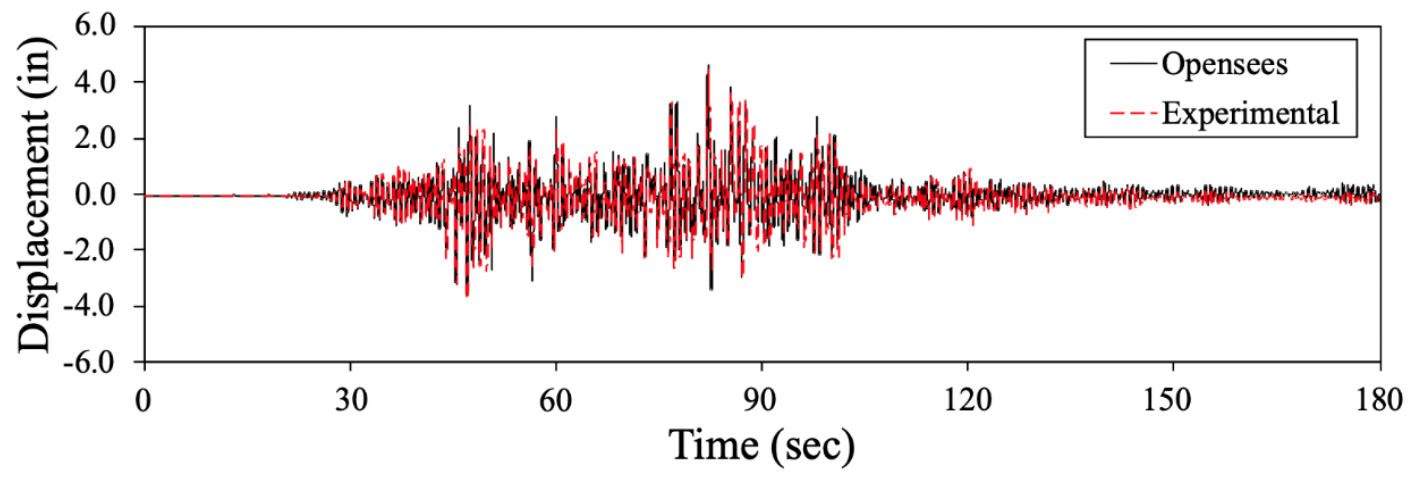

b)

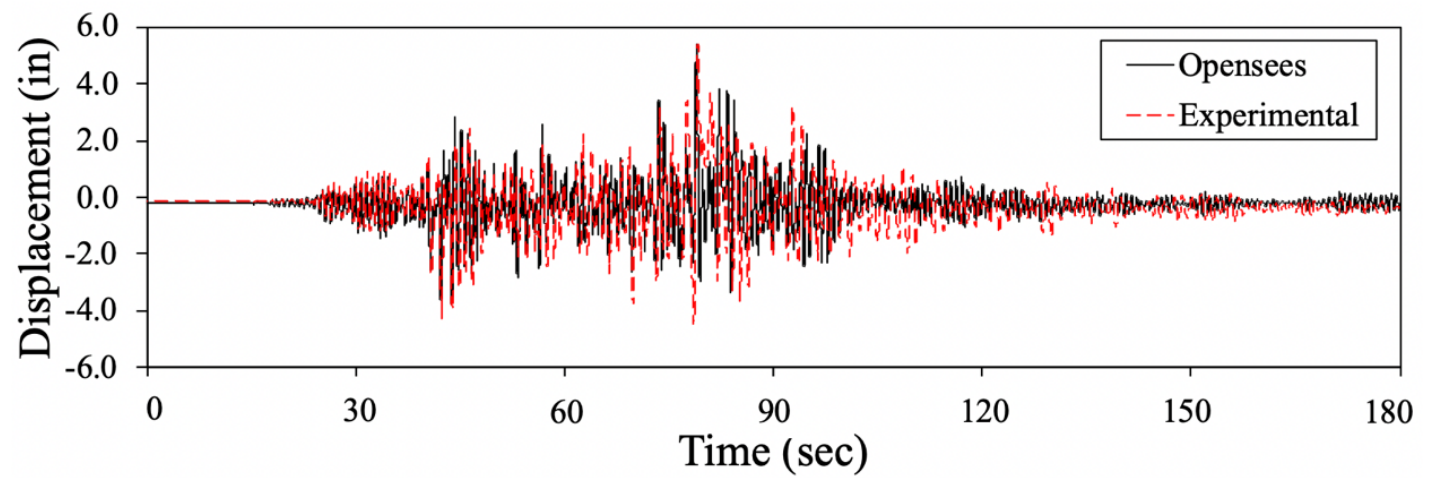

c)

Figure 0.2 Displacement histories comparison for Column C2C-M for all amplitude motions 


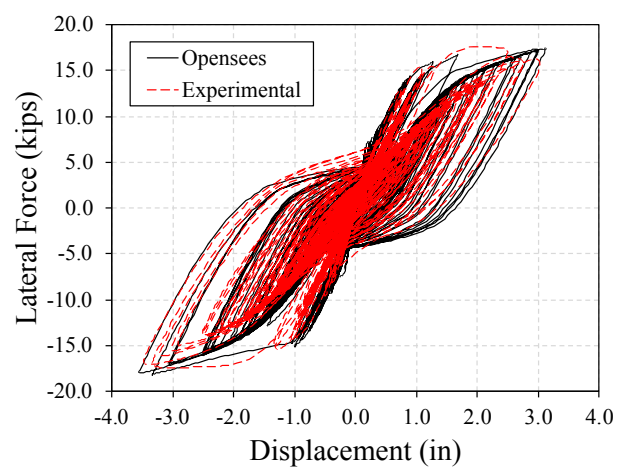

a)

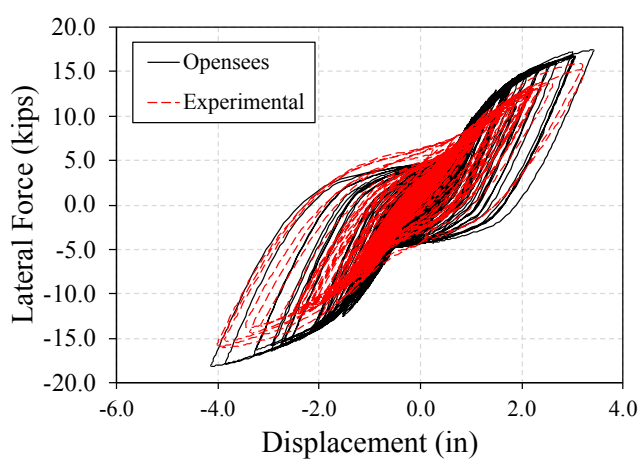

b)

Figure 0.3 Hysteresis curve comparison for Column C32C-T for all amplitude motions

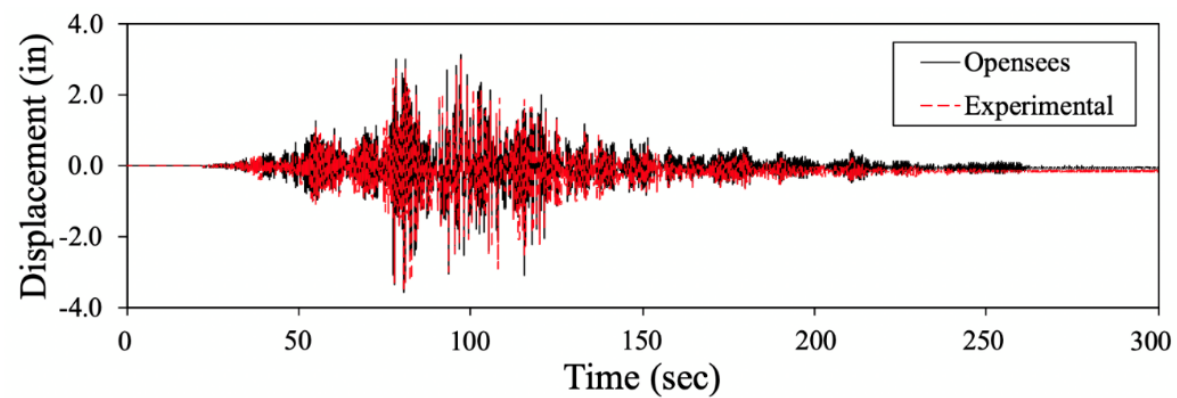

a)

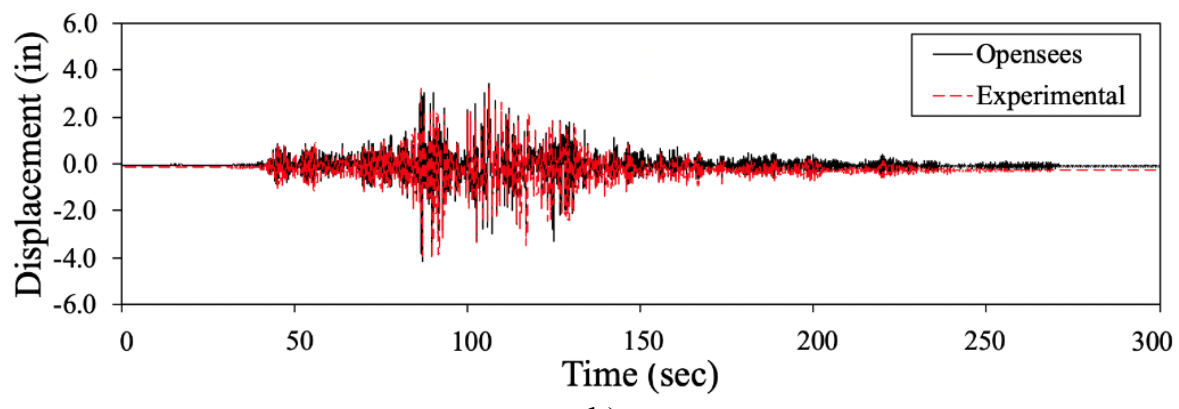

b)

Figure 0.4 Displacement histories comparison for Column C3C-T. a) Amp.A, b) Amp.B 


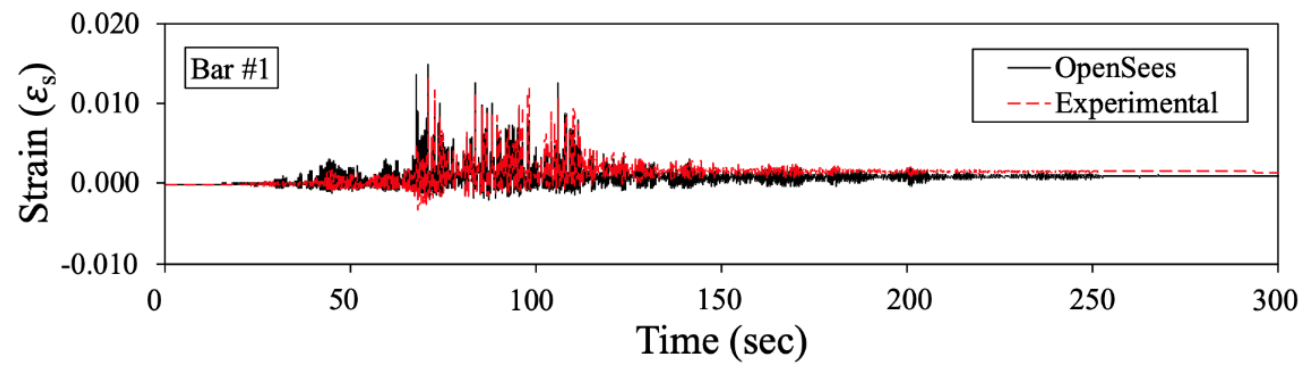

a)

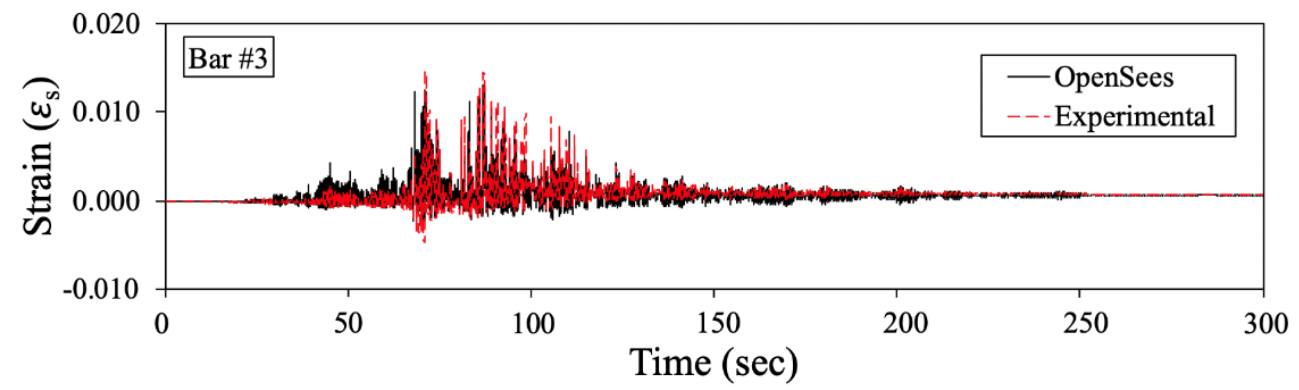

b)

Figure 0.5 Steel strain histories comparison for Column C3C-T in Amp.A. a) Bar\#1, b) Bar\#3

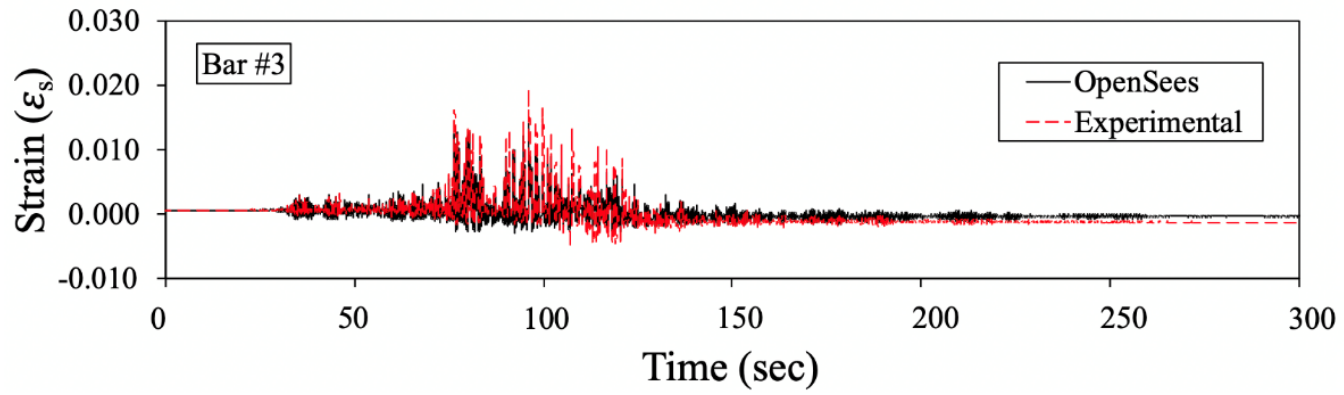

a)

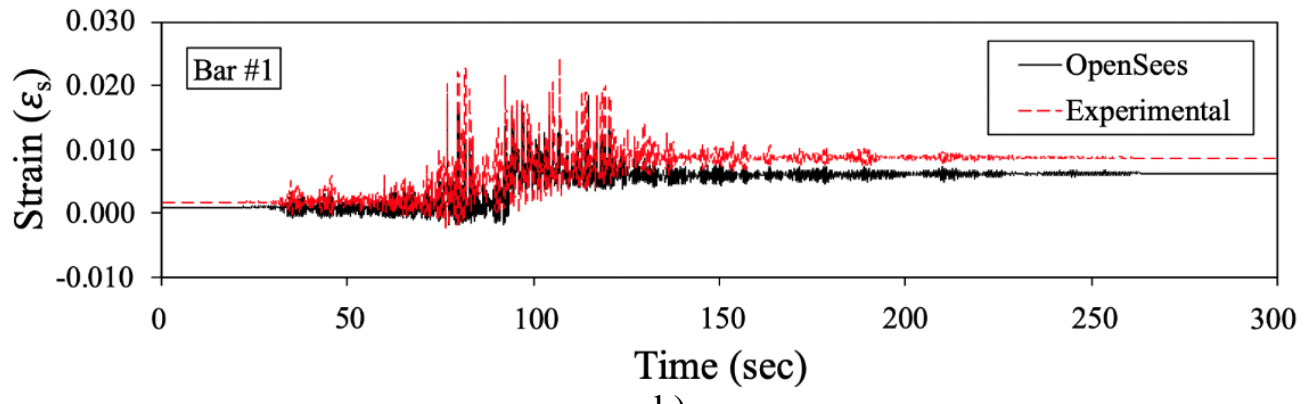

b)

Figure 0.6 Steel strain histories comparison for Column C3C-T in Amp.B. a) Bar\#1, b) Bar\#3 


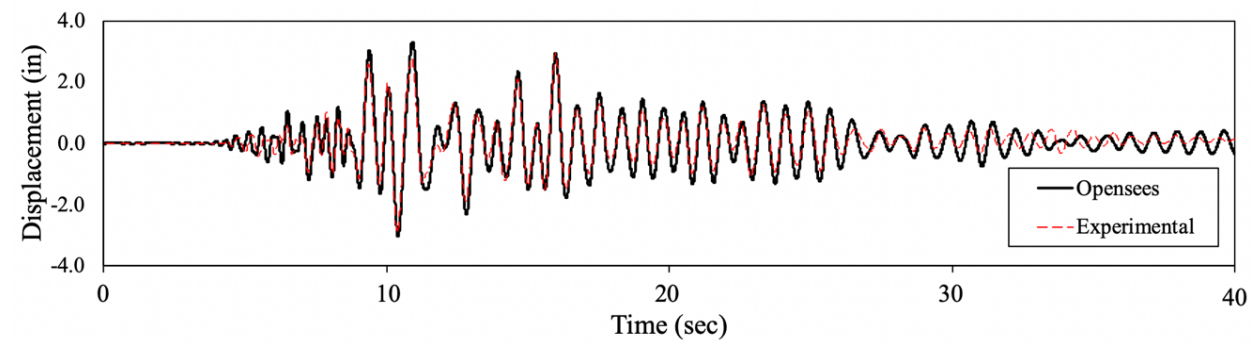

a)

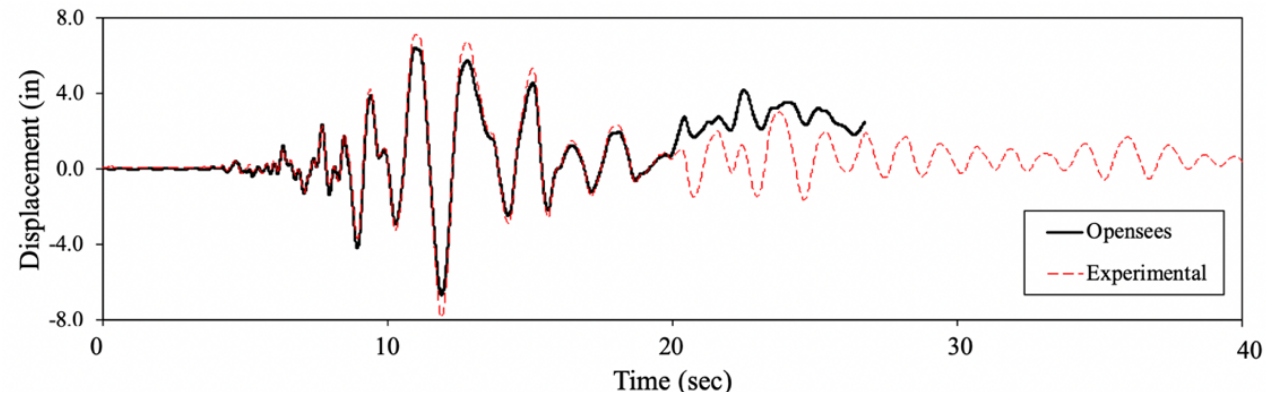

b)

Figure 0.7 Displacement histories comparison for Column C4S1-L. a) Amp.A, b) Amp.B

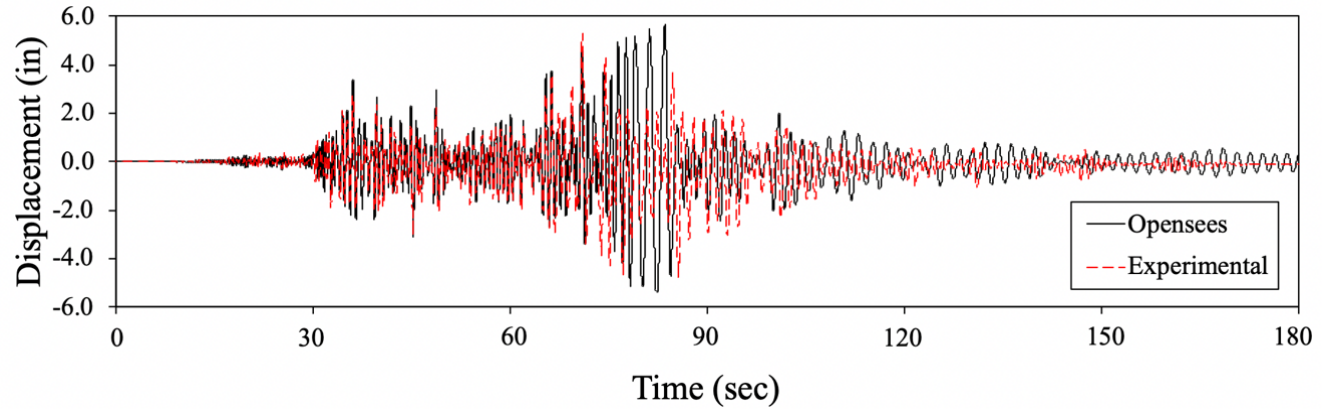

Figure 0.8 Displacement histories comparison for Column C5S1-M during Amp.A

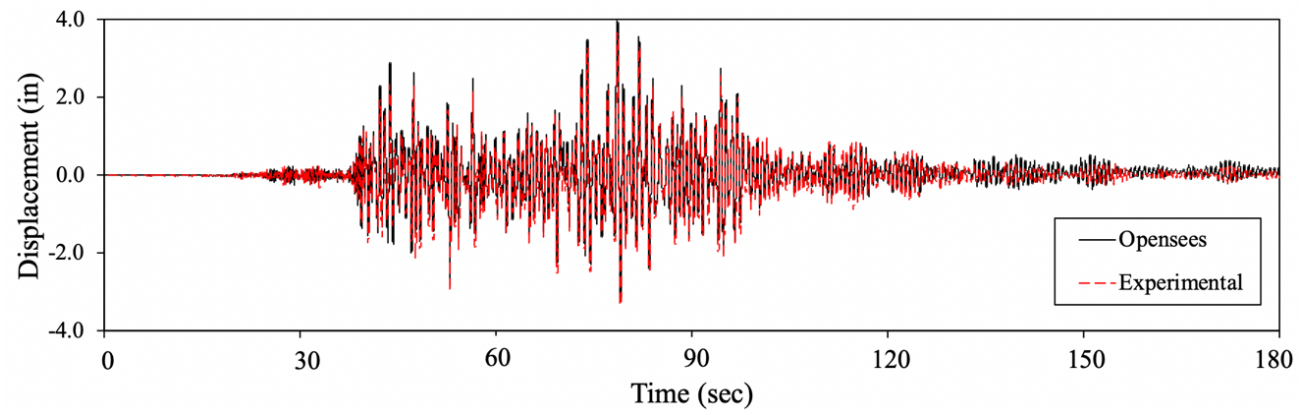

Figure 0.9 Displacement histories comparison for Column C6S2-M during Amp.A 